
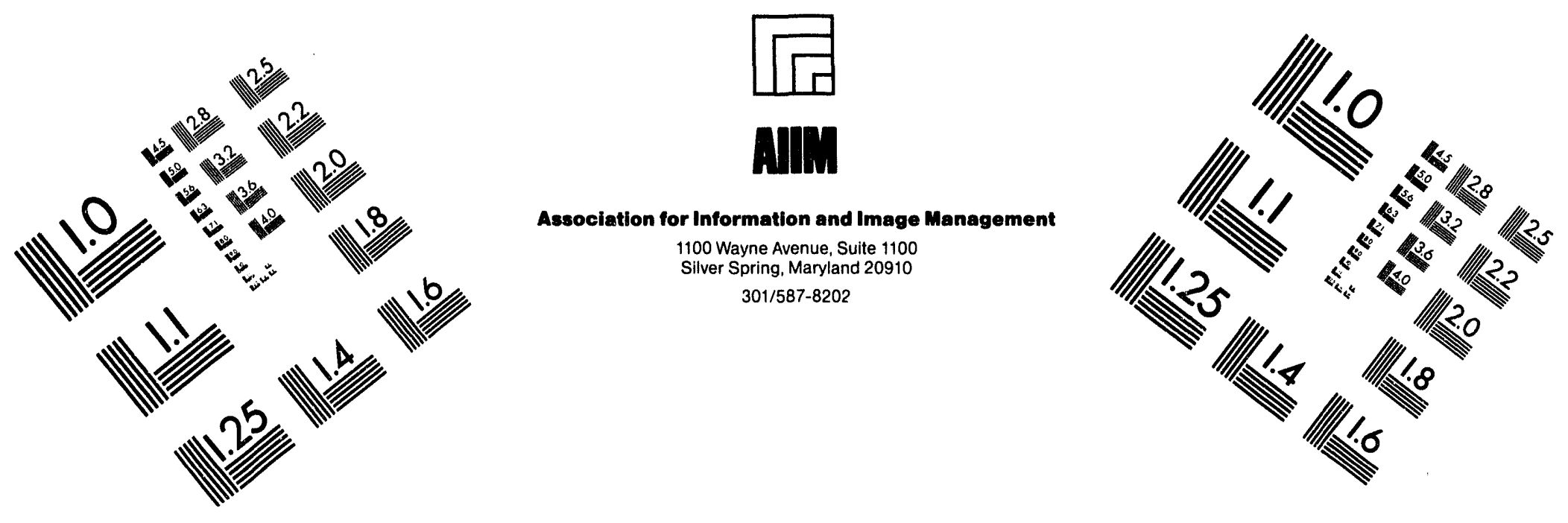

\title{
Centimeter
}

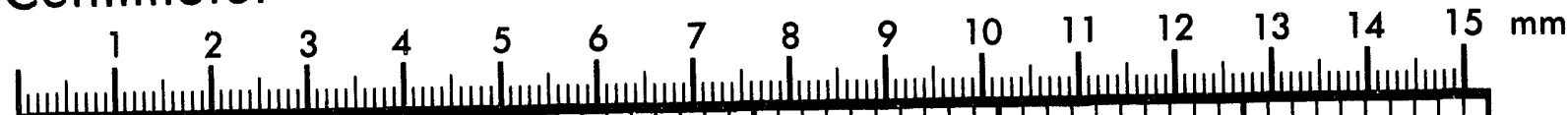

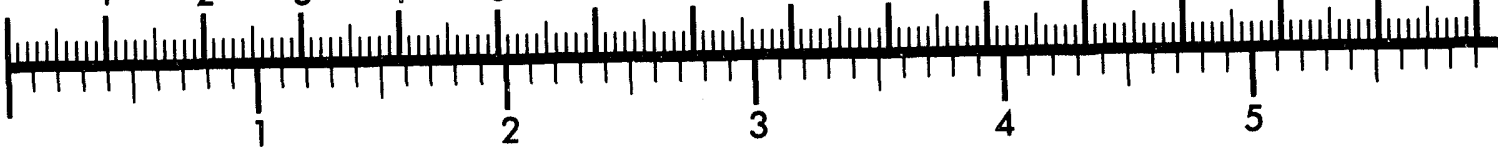
Inches
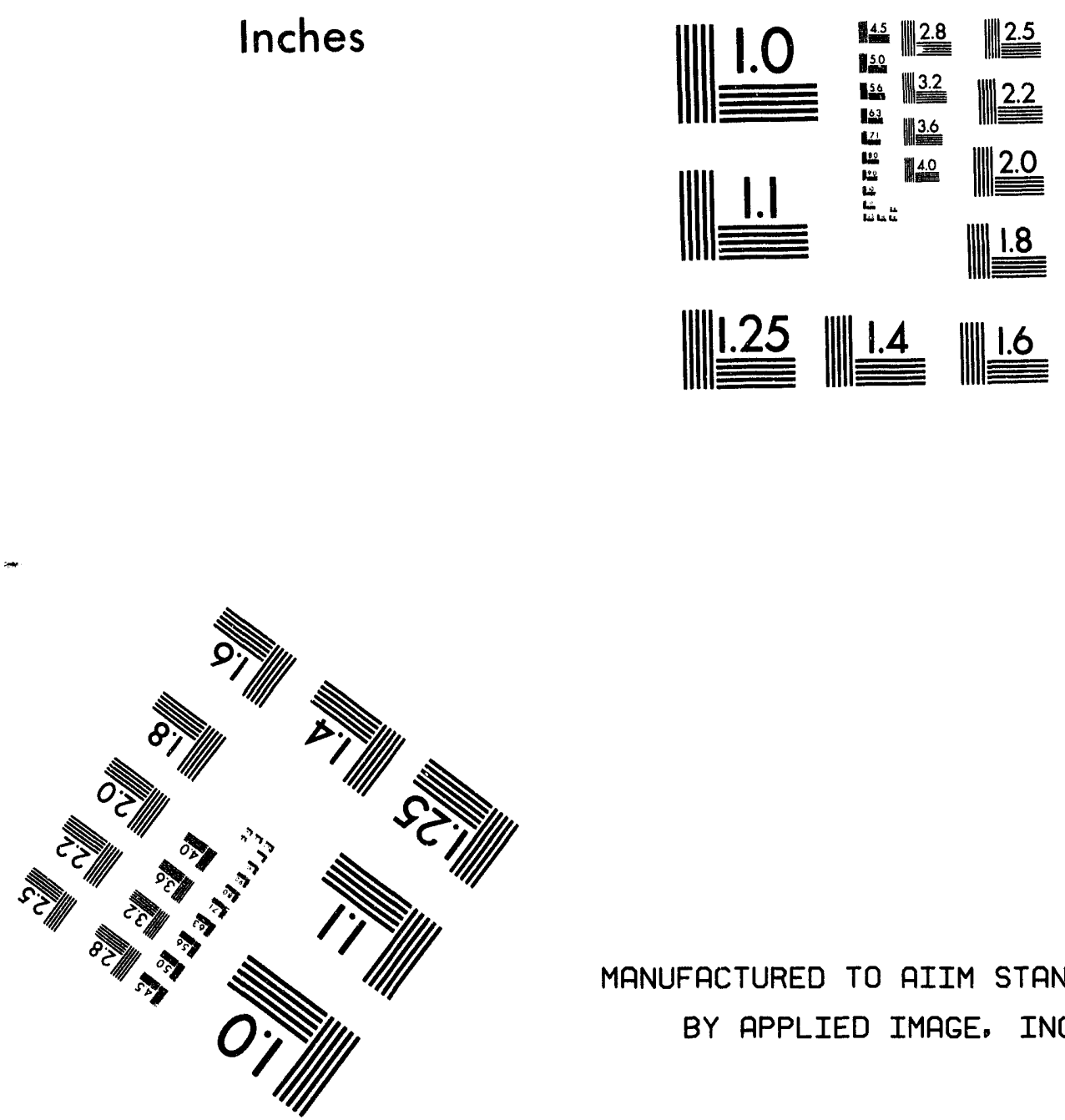

MANUFACTURED TO AIIM STANDARDS BY APPLIED IMAGE. INC.

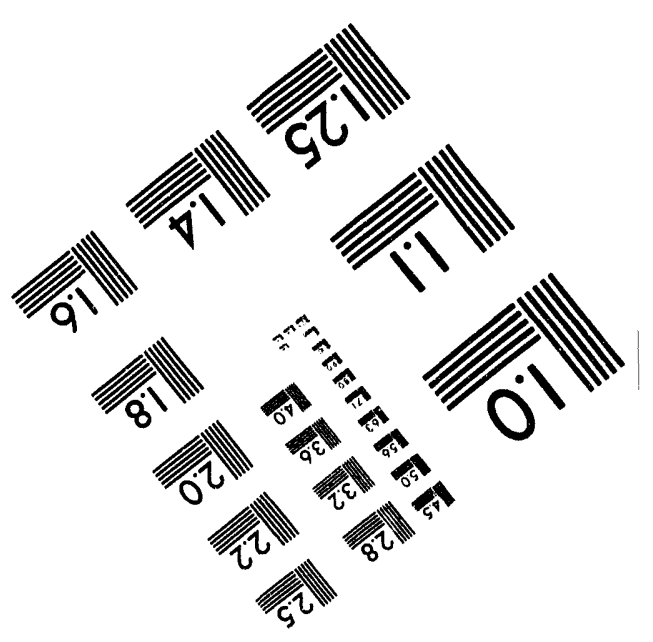



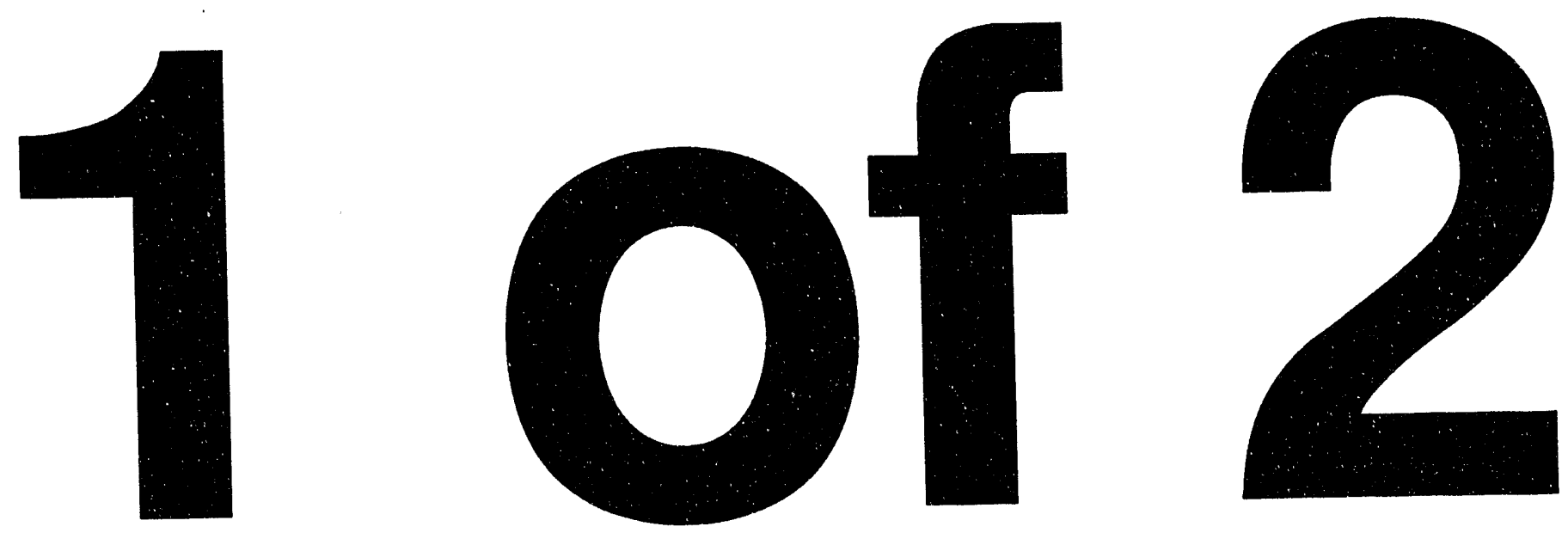


\begin{tabular}{|l|l|}
\hline $\begin{array}{l}\text { 2. To: (Receiving Organization) } \\
\text { Distribution }\end{array}$ & $\begin{array}{l}\text { 3. From: Coriginating Organization) } \\
\text { 300 Area Source Investigation } \\
\text { \& Remediation Projects }\end{array}$ \\
\hline $\begin{array}{l}\text { 5. Proj./Prog./Dept./Div.: } \\
\text { 300-FF-2 Remedial } \\
\text { Investigation }\end{array}$ & $\begin{array}{l}\text { 6. Cog. Engr.: } \\
\text { L.C. Hulstrom }\end{array}$ \\
\hline
\end{tabular}

8. Originator Remarks:

Release to Record File and Distribution List
4. Related EDT No.:

$$
N / A
$$

7. Purchase Order No.:

$N / A$

11. Receiver Remarks:

9. Equip./Component No.:

$N / A$

10. System/Bldg./Facility:

$N / A$

12. Major Assm. Dwg. No.:

$N / A$

13. Permit/Permit Application No.: $N / A$

14. Required Response Date: None

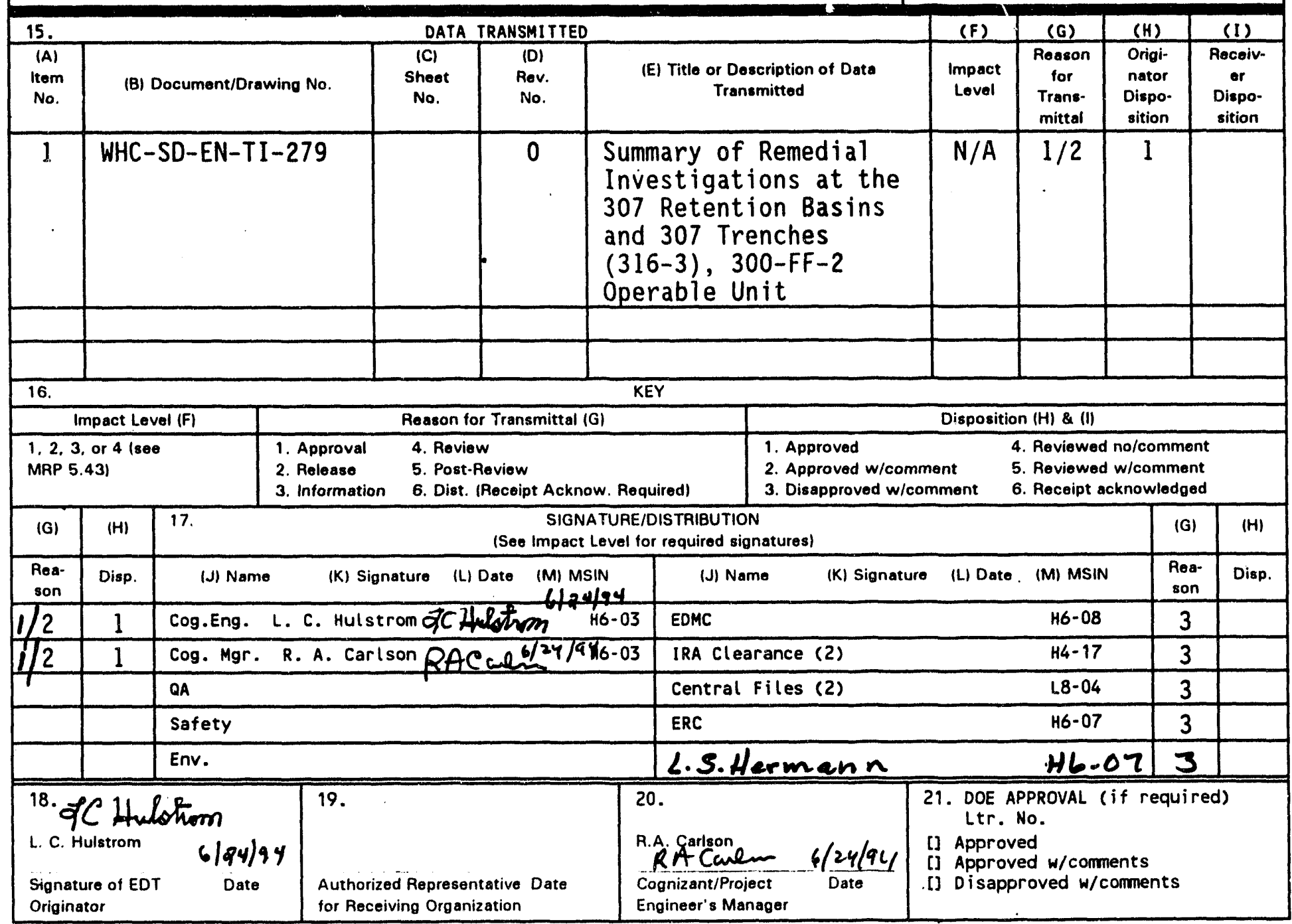




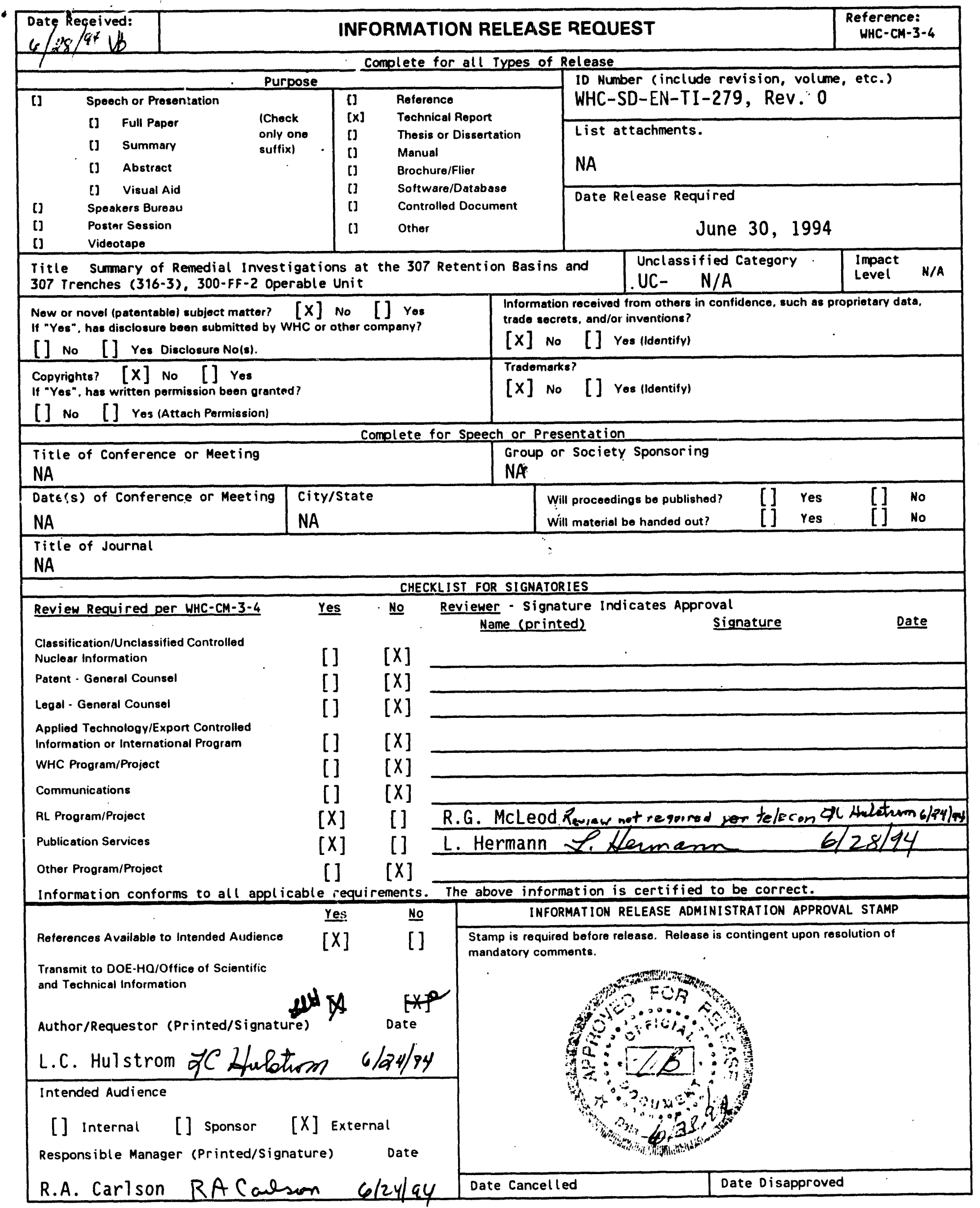




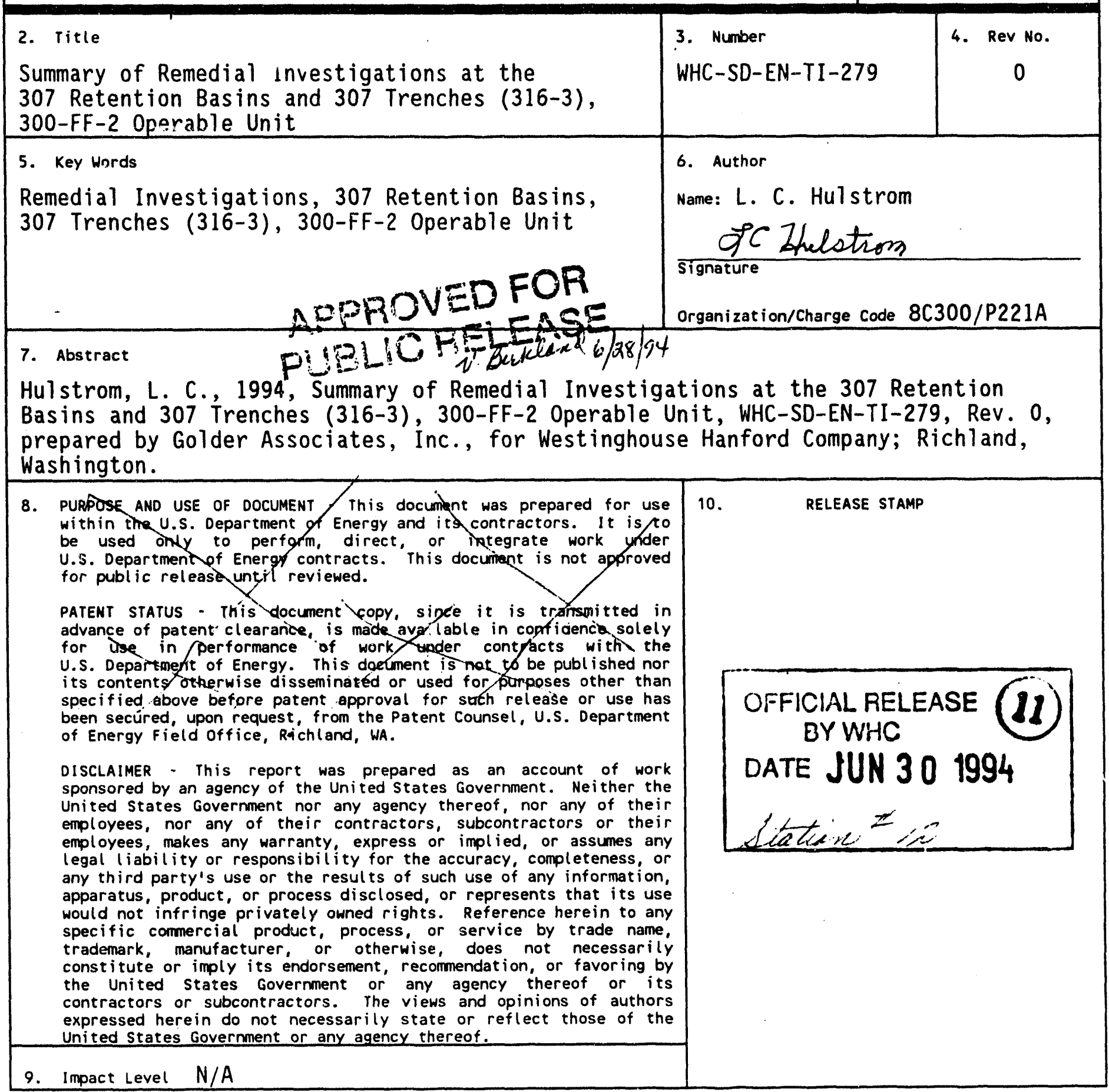


WHC-SD-EN-TI-279, Rev. 0

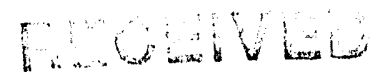

J日L 28193

OSTI

\section{SUMMARY OF REMEDIAL INVESTIGATIONS AT THE 307 RETENTION BASINS AND 307 TRENCHES (316-3), 300-FF-2 OPERABLE UNIT}

\section{DISCLAIMER}

This report was prepared as an account of work sponsored by an agency of the United States Government. Neither the United States Government nor any agency thereof, nor any of their employees, makes any warranty, express or implied, or assumes any legal liability or responsjbility for the accuracy, completeness, or usefulness of any information, apparatus, product, or process disclosed, or represents that its use would not infringe privately owned rights. Reference herein to any specific commercial product, process, or service by trade name, trademark, manufacturer, or otherwise does not necessarily constitute or imply its endorsement, recommendation, or favoring by the United States Government or any agency thereof. The views and opinions of authors expressed herein do not necessarily state or reflect those of the United States Government or any agency thereof. 
1.0 INTRODUCTION $\ldots \ldots \ldots \ldots \ldots \ldots \ldots \ldots \ldots \ldots \ldots \ldots \ldots \ldots \ldots \ldots \ldots$

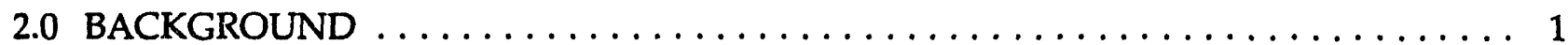

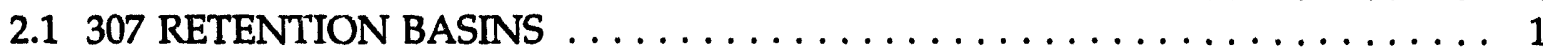

2.2307 TRENCHES . . . . . . . . . . . . . . . . . . . . . . 2

3.0 SUMMARY OF REMEDIAL INVESTIGATIONS $\ldots \ldots \ldots \ldots \ldots \ldots \ldots \ldots \ldots \ldots$

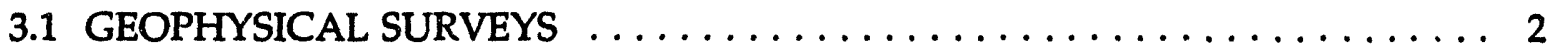

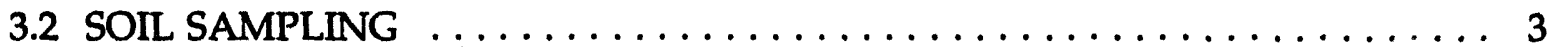

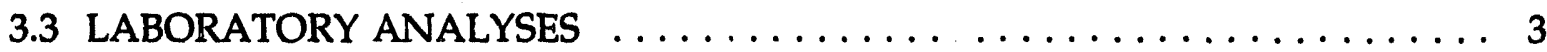

4.0 REFERENCES $\ldots \ldots \ldots \ldots \ldots \ldots \ldots \ldots \ldots \ldots \ldots \ldots \ldots \ldots \ldots \ldots \ldots$

\section{FIGURES:}

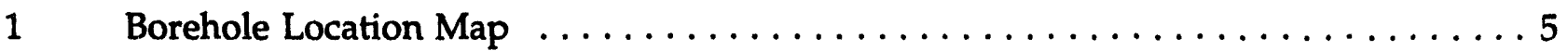

\section{TABLES:}

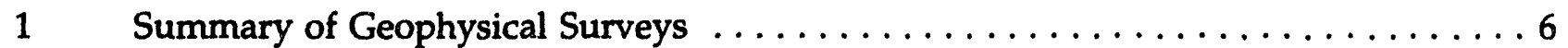

2 Summary of Remedial Investigation Boreholes $\ldots \ldots \ldots \ldots \ldots \ldots \ldots \ldots \ldots$

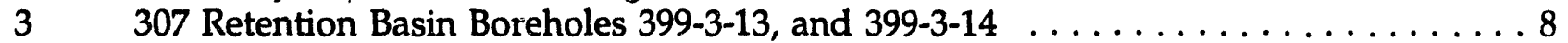

$4 \quad 307$ Trenches Boreholes 399-3-15, 399-3-16, and 399-3-17 . . . . . . . . . . 10

\section{APPENDDX:}

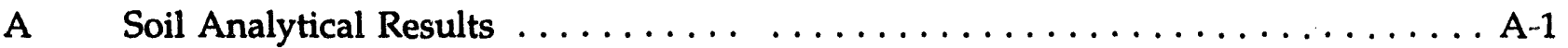




\subsection{INTRODUCTION}

Remedia! investigations at the 307 retention basins and 307 trenches (316-3) in the 300 Area of the Hanford Site were conducted as part of the 300-FF-1 operable unit Phase I remedial investigation (RI) in accordance with the approved RI work plan (DOE-RL 1990). During the RI, the southwestern boundary of the 300 . FF-1 operable unit was modified by all signatories to the Hanford Federal Facility Agreement and Consent Order (Ecology et al. 1989), which shifted the 307 retention basins and 307 trenches to the 300-FF-3 operable unit. As a consequence, the RI results from these waste management units were not included in the Phase I Remedial Investigation Report for the 300-FF-1 Operable Unit (DOE-RL 1993).

As a result of recent Hanford Federal Facility Agreement and Consent Order negotiations, the 300-FF-2 operable unit now consists of the remaining 300 Area orable units within the 300 Area National Priorities List (NPL), which includes the former 300-FF-3 operable unit. Therefore, this document summarizes the RI results from the 307 retention basins and 307 trenches in the 300-FF-2 operable unit. Analysis and evaluation of these results will be included in the $300-$ FF-2 RI report.

\subsection{BACKGROUND}

The 307 retention basins and the 307 trenches (316-3) are located in the 300 Area of the Hanford Site, south of the 340 complex and 300 Area ash pits, respectively (Figure 1). Prior to 1953, laboratory amounts of radioactive wastes generated in the 300 Area facilities were discharged to the process sewer system. In 1953, the 307 retention basins and 307 trenches were built, and thereafter, all radioactive wastes were handled by this system. In 1963, discharge to the 307 trenches was eliminated, and the low level radioactive discharges were transferred back to the process sewer system.

\subsection{RETENTION BASINS}

The 307 retention basins were constructed in 1953, along with the 307 trenches, to accommodate the expansion of laboratory facilities in the 300 Area. They are located just south of the 340 complex (Figure 1). Buildings in the 300 Area which have connections to the 307 retention basins include the 308 fuels development laboratory, 325 radiochemistry laboratory, 326 materials technology laboratory, 327 post-irradiation laboratory, 328 mechanical development building, and 329 biophysics laboratory (DOE-RL 1990).

The 307 retention basins consist of four 94,600-L (25,000 gal) concrete retention basins where laboratory wastewaters, consisting of cooling water, seal water, laboratory and test facility wastes, can be held for analysis. If analysis showed the wastes to be below acceptable radiological discharge limits for ground disposal, the wastes were transferred by pipe to the 307 trenches. After the 307 trenches were retired in 1963, these wastes were routed to the 
307 trenches. After the 307 trenches were retired in 1963, these wastes were routed to the process sewer system. If the wastes were determined to be above the radiological discharge limits, they were transferred to the 340 complex where the waste was stored before being transported to the 200 West Area for storage or disposal. The acceptable discharge limits were $5,000 \mathrm{pCi} / \mathrm{L}$ and $1,000 \mathrm{pCi} / \mathrm{L}$ of total alpha and beta activity, respectively. The 307 retention basins are still in use.

Because of difficulties with sampling, about $40 \%$ of the wastes received by the 307 retention basins between 1953 and 1963 were released to the 307 trenches without sanpling. As a result of this practice, it is possible that wastes exceeding discharge limits were unknowingly released into the 307 trer ches. The available data for the period from 1953 through 1960, however, indicate that the discharge limits were not exceeded. Only radiological monitoring was performed. The chemical nature of the wastes was never determined (DOE-RL 1990).

\subsection{TRENCHES}

The 307 trenches, or 316-3 laboratory waste disposal trenches, were two parallel, leaching trenches located southwest of the 300 Area ash pits, within the 300 Area perimeter fence (Figure 1). Each trench was 152 by 6 by $6 \mathrm{~m}$ deep ( 500 by 20 by $20 \mathrm{ft}$ deep). Flows to the trenches ranged from 113,550 to $378,000 \mathrm{~L} / \mathrm{d}(30,000$ to $100,000 \mathrm{gal} / \mathrm{d})$ (DOE-RL 1990).

The trenches were constructed in 1953 in conjunction with the 307 retention basins and were the recipients of the low level liquid waste from the retention basins. These trenches were retired in 1963 and excavated. The bulk of the contaminated soils were disposed in the 300 north burial ground. In 1965, the trenches were backfilled with contaminated soils from the south process pond (316-1). These soils were then covered with fly ash from the 300 Area ash pits. This facility currently has a gravel surface, except where two storage buildings ( $3718 \mathrm{E}$ and $3718 \mathrm{G}$ ) occupy areas above the old trenches.

\subsection{SUMMARY OF REMEDIAL INVESTIGATIONS}

\subsection{GEOPHYSICAL SURVEYS}

The Automation and Measurement Sciences Department at Pacific No thwest Laboratory (PNL) conducted ground-penetrating radar (GPR) and electromagn itic inductance (EMI) surveys at these two waste management units in 1990. The GPR surveys were conducted along north-south and east-west oriented grid lines spaced $1 \mathrm{~m}(3.3 \mathrm{ft}$; apart and the EMI surveys were conducted along north-south oriented lines spaced $2 \mathrm{~m}(6.4 \mathrm{ft})$ apart.

The south and east sides of the 307 retention basins were geophysically surveyed using GPR (Sandness 1991a). Several pipelines were located in this area. Three pipelines running in a north-south direction on the east side of the basins are believed to be a segment of the active process sewer $(3.4 \mathrm{~m}$ [11 ft] deep) and two segments of the retired radioactive liquid waste sewer $(1.2 \mathrm{~m}[4 \mathrm{ft}]$, and $1.5 \mathrm{~m}$ [ $5 \mathrm{ft}$ ] deep). These pipelines are 0.6 to $0.9 \mathrm{~m}$ 
( 2 to $3 \mathrm{ft}$ ) east of the locations shown on site drawings. The process sewer is believed to be located south of the basins and buried $3 \mathrm{~m}(9 \mathrm{ft})$ deep. Other unidentified pipelines are also located in this area.

A subsequent GPR survey (Sandness 1991b) was performed in an area immediately west of the 307 retention basins to detect underground obstructions that could constitute hazards during the borehole drilling. The GPR survey detected several underground pipes and many localized metallic objects. While some pipes were detected as deep as $1.8 \mathrm{~m}(6 \mathrm{ft})$, most were generally less than $0.6 \mathrm{~m}(2 \mathrm{ft})$ depth. Table 1 provides the results of the geophysical survey.

An EMI geophysical survey of the 307 trench area, to assist in confirming the trench boundaries, was hampered by the presence of buildings, fences, and surface debris (Sandness 1991b). While pipelines and possible cables were detected in the GPR survey, an accurate determination of the trench boundaries was not possible. Table 1 summarizes the results of the geophysical survey.

\subsection{SOIL SAMPLING}

Soil sampling activities were conducted at these two waste management units from October 1991 to January 1992. The drilling activities and soil sampling investigation at these waste management units are described in Weekes (1992) and are briefly summarized below. Borehole locations are shown on Figure 1 and Table 2 summarizes the RI borehole data for these two waste management units.

Two vertical boreholes were completed and sampled to characterize the 307 retention basins. One borehole was located on the west side and one on the east side of the basins.

Three boreholes were completed and sampled to characterize the 307 trenches, since the trenches were completely excavated, including removal of the center berm area, and backfilled with other material.

\subsection{LABORATORY ANALYSES}

Soil samples collected from the RI boreholes were analyzed by Thermo-Analytical Laboratories (TMA), Weston Analytics and Teledyne Isotopes (under contract to Weston) for organics, inorganics (metals and cyanide), general chemistry and radionuclides. The analytical results were validated by Ebasco Services Company and Hart-Crowser Company as described in WHC (1992a,b,c,d,e,f). The RI analytical results for these two waste management units, along with the data qualifiers from the data validation reports, are presented in Appendix A. Selected soil samples were analyzed by the Westinghouse Hanford Company geotechnical laboratory for physical properties; these data are summarized in Weekes (1992).

Tables 3 and 4 provide a summary listing of the RI borehole soil samples that were submitted for laboratory analyses. 


\subsection{REFERENCES}

DOE-RL, 1990, Remedial Investigation/Feasibility Study Work Plan for the 300-FF-1 Operable Unit, Hanford Site, Richland, Washington, DOE/RL 88-31, Rev. 0, U.C. Department of Energy, Richland, Washington.

DOE-RL, 1993, Phase I Remedial Investigation Report for the 300-FF-1 Operable Unit, DOE/RL-92-43, Rev. 0, U.S. Department of Energy, Richland, Washington.

Ecology, EPA, and DOE-RL, 1989, et seq., Hanford Federal Facility Agreement'and Consent Order, Washington Department of Ecology, Olympia, Washington, U.S. Environmental Protection Agency, Region X, Seattle, Washington, and U.S. Department of Energy, Richland Operations Office, Richland, Washington.

Sandness, G.A., 1991a, Report on Geophysical Surveys in the 300-FF-1 Operable Unit, EMO-1032, Pacific Northwest Laboratories, Environmental Management Operations, Richland, Washington.

Sandness, G.A., 1991b, Geophysical Surveys, Pacific Northwest Laboratory, Richland, Washington.

Weekes, D.C., 1992, Summary of Drilling and Test Pit Activities for the 300-FF-1 Operable Unit, Phase 1 Soil Sampling Investigation, WHC-SD-EN-TI-38, Westinghouse Hanford Company, Richland, Washington.

WHC, 1992a, Data Validation and Limitations Report, Number 1, WHC-SD-EN-TI-041, Rev. 0, Prepared by Ebasco Services Company and Hart Crowser Company for Westinghouse Hanford Company, Richland, Washington.

WHC, 1992b, Data Validation and Limitations Report, Number 2, WHC-SD-EN-TI-042, Rev. 0, Prepared by Ebasco Services Company and Hart Crowser Company for Westinghouse Hanford Company, Richland, Washington.

WHC, 1992c, Data Validation and Limitations Report, Number 3, WHC-SD-EN-TI-043, Rev. 0, Prepared by Ebasco Services Company and Hart Crowser Company for Westinghouse Hanford Company, Richland, Washington.

WHC, 1992d, Data Validation and Limitations Report, Number 5 , WHC-SD-EN-TI-045, Rev. 0, Prepared by Ebasco Services Company and Hart Crowser Company for Westinghouse Hanford Company, Richland, Washington.

WHC, 1992e, Data Validation and Limitations Report, Number 6, WHC-SD-EN-TI-046, Rev. 0, Prepared by Ebasco Services Company and Hart Crowser Company for Westinghouse Hanford Company, Richland, Washington.

WHC, 1992f, Data Validation and Limitations Summary, WHC-SD-EN-TI-047, Rev. 0, Prepared by Ebasco Services Company and Hart Crowser Company for Westinghouse Hanford Company, Richland, Washington. 
WHC-SD-EN-TI-279, Rev. 0

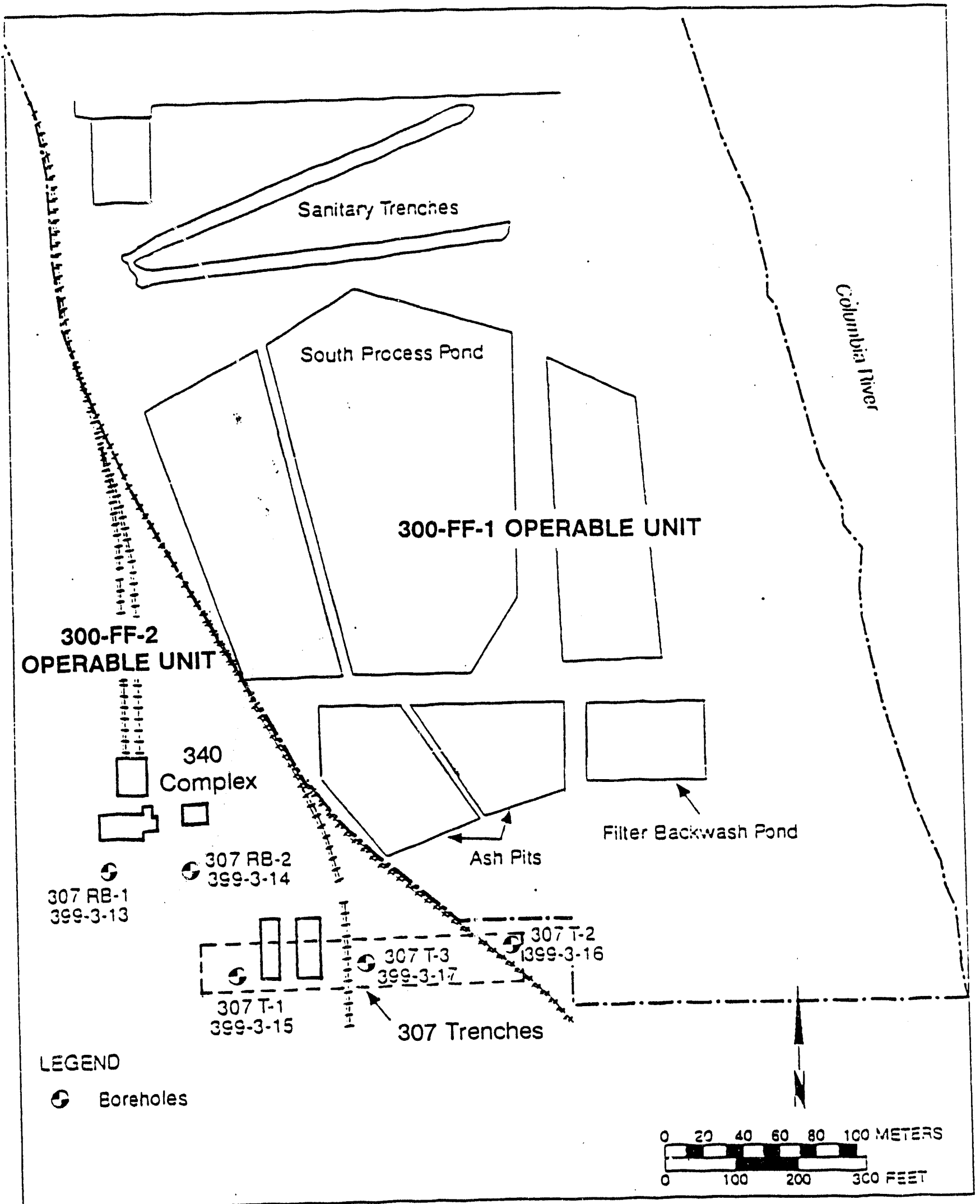

Figure 1. Borehole Location Map. 
WHC-SD-EN-TI-279, Rev. 0

Table 1. Summary of Geophysical Surveys.

\begin{tabular}{|l|l|l|l|}
\hline \multicolumn{1}{|c|}{ Main target } & \multicolumn{1}{|c|}{ Target description } & $\begin{array}{c}\text { Survey } \\
\text { methods }\end{array}$ & Comments \\
\hline $\begin{array}{l}\text { South and east sides of the } \\
307 \text { retention basins }\end{array}$ & $\begin{array}{l}\text { high complexity of the } \\
\text { subsurface piping around } \\
\text { the basins }\end{array}$ & GPR & $\begin{array}{l}\text { Several pipes were detected. The three south- } \\
\text { north pipes located west c! the basins are } \\
\text { assumed to be (west to east): a segment of } \\
\text { the active process sewer (11 ft deep), the } \\
\text { abandoned radioactive liquid waste sewer (4 } \\
\text { ft deep) and the abandoned radioactive liquid } \\
\text { waste sewer from the 309 building (5 ft deep). } \\
\text { Some sections of the active process sewer, } \\
\text { converging at a manhole could be detected. } \\
\text { Several other east-west and north-south pipe } \\
\text { segments appear also in the GPR profiles. }\end{array}$ \\
\hline $\begin{array}{l}\text { Area immediately west of } \\
307 \text { retention basins }\end{array}$ & subsurface piping & $\begin{array}{l}\text { GPR detected several underground pipes and } \\
\text { many localized metallic objects. The pipes } \\
\text { were generally at less than } 2 \mathrm{ft} \text { depth. }\end{array}$ \\
\hline 307 Trenches & GPR & $\begin{array}{l}\text { Buildings, fences, and surface clutter present } \\
\text { at the site prevented access to major portions } \\
\text { of the site area. EM sensor rendered virtually } \\
\text { useless while pipelines and possible cables } \\
\text { were detected, an accurate determination of } \\
\text { the trench boundaries was not possible. }\end{array}$ \\
\hline
\end{tabular}

Source: Sandness 1991a, b

EMI - Electro Magnetic Inductance

GPR - Ground Penetrating Radar 
Table 2. Summary of Remedial Investigation Boreholes.

\begin{tabular}{|c|c|c|c|c|c|c|c|c|c|c|}
\hline \multirow[t]{2}{*}{ Location } & \multirow[t]{2}{*}{$\begin{array}{l}\text { Boring or } \\
\text { Test Pit * }\end{array}$} & \multicolumn{2}{|c|}{$\begin{array}{l}\text { Coordinates } \\
\text { Nad } 83(\mathrm{~m})\end{array}$} & \multicolumn{2}{|c|}{$\begin{array}{c}\text { Depth of } \\
\text { Temp Casing }\end{array}$} & \multirow[t]{2}{*}{ Start Date } & \multirow[t]{2}{*}{ End Date } & \multirow[t]{2}{*}{$\begin{array}{l}\text { Total Depth } \\
\text { (ft) }\end{array}$} & \multirow[t]{2}{*}{$\begin{array}{l}\text { Depth to } \\
\text { Water (Ft) }\end{array}$} & \multirow[t]{2}{*}{$\begin{array}{l}\text { Ground Elev. } \\
\text { (ft) }\end{array}$} \\
\hline & & Northing & Easting & $10^{\prime \prime}$ & $8^{\prime \prime}$ & & & & & \\
\hline \multirow[t]{3}{*}{307 Trenches } & 399-3-15 & 115853.80 & 594211.95 & $25^{\prime}$ & $65^{\prime}$ & $1 / 2 / 92$ & $1 / 15 / 92$ & 68 & 54.7 & 396.73 \\
\hline & 399-3-16 & 115867.60 & 594350.32 & 25.77 & $58^{\circ}$ & $12 / 2 / 91$ & $12 / 13 / 91$ & 60 & 49.4 & 390.86 \\
\hline & 399-3-17 & 115857.72 & 594274.58 & $25.5^{\prime}$ & $63.3^{\prime}$ & $1 / 20 / 92$ & $1 / 29 / 92$ & 65 & 51 & 393.96 \\
\hline \multirow{2}{*}{$\begin{array}{l}307 \text { Retention } \\
\text { Basin }\end{array}$} & 399-3-13 & 115910.61 & 594146.58 & $15^{\prime}$ & $64^{\circ}$ & $10 / 18 / 91$ & $11 / 5 / 91$ & 67 & 44 & 386.92 \\
\hline & 399-3-14 & 115908.68 & 594188.85 & $15^{\prime}$ & $54^{\prime}$ & $\cdot 11 / 11 / 91$ & $11 / 21 / 91$ & 56 & 47 & 388.86 \\
\hline
\end{tabular}


WHC-SD-EN-TI-279, Rev: 0

Table 3. 307 Retention Basin Boreholes 399-3-13, and 399-3-14. (Sheet 1 of 2)

\begin{tabular}{|c|c|c|c|c|c|c|c|}
\hline $\begin{array}{l}\text { Sample } \\
10\end{array}$ & $\begin{array}{l}\text { Sample } \\
\text { Date }\end{array}$ & $\begin{array}{l}\text { Top of } \\
\text { Sample } \\
\text { (t) }\end{array}$ & $\begin{array}{l}\text { Ectiom of } \\
\text { Sample } \\
(f t)\end{array}$ & $\begin{array}{l}\text { Physical } \\
\text { Analysis }\end{array}$ & $\begin{array}{l}\text { Chemical } \\
\text { Analysis }\end{array}$ & $\begin{array}{c}\text { Sample } \\
\text { Archived }\end{array}$ & Comments \\
\hline \multicolumn{8}{|c|}{$\begin{array}{l}307 \text { Retention Basin } \\
\text { Borehole: } 307 R 8-1,399-3-13\end{array}$} \\
\hline 801409 & $10 / 18 / 91$ & 0.0 & 2.0 & & $\bar{x}$ & \multirow{20}{*}{$x$} & \multirow{7}{*}{ Split to EO14R0 } \\
\hline 801480 & $10 / 18 / 91$ & 5.0 & 7.5 & & $x$ & & \\
\hline BO14R1 & $10 / 18 / 91$ & 5.0 & 7.5 & & $x$ & & \\
\hline E015Y8 & 10/18/91 & 5.0 & 7.5 & $x$ & & & \\
\hline E014R2 & $10 / 21 / 99$ & 8.0 & 10.5 & & $x$ & & \\
\hline $8014 R 3$ & $10 / 21 / 91$ & 11.0 & 13.0 & & $x$ & & \\
\hline BO14R4 & $10 / 24 / 91$ & 15.0 & 17.0 & & $x$ & & \\
\hline E014R5 & $10 / 24 / 91$ & 22.0 & 23.0 & & $x$ & & \\
\hline BO14R6 & $10 / 25 / 91$ & 25.0 & 27.0 & & $x$ & & Duplicate \\
\hline$B 014 A 7$ & $10 / 25 / 91$ & 25.0 & 27.0 & & $x$ & & Duplicate \\
\hline BO15Y9 & $10 / 25 / 91$ & 25.0 & 27.0 & $x$ & & & \\
\hline $8014 R 8$ & $10 / 25 / 91$ & 30.0 & 32.0 & & $x$ & & \\
\hline $8015 Z 0$ & $10 / 25 / 91$ & 33.0 & 35.0 & & & & \\
\hline $8014 R 9$ & $10 / 25 / 91$ & 35.0 & 37.0 & & $x$ & & \\
\hline 801450 & $10 / 25 / 91$ & 42.0 & 44.0 & & $x$ & & \\
\hline $8015 Z 1^{\circ}$ & $10 / 28 / 91$ & 47.0 & 49.0 & $x$ & & & \\
\hline 801451 & $10 / 28 / 91$ & & & & $x$ & & Field blank \\
\hline B014S2 & $10 / 28 / 91$ & . & & & $x$ & & Field blank \\
\hline 801453 & $10 / 28 / 91$ & & & & $x$ & & Field blank \\
\hline$B 01454^{\circ}$ & $11 / 04 / 91$ & 65.0 & 67.0 & & $x$ & & \\
\hline
\end{tabular}


Table 3. 307 Retention Basin Boreholes 399-3-13, and 399-3-14. (Sheet 2 of 2)

\begin{tabular}{|c|c|c|c|c|c|c|c|}
\hline $\begin{array}{l}\text { Sample } \\
\text { ID }\end{array}$ & $\begin{array}{l}\text { Sample } \\
\text { Date }\end{array}$ & $\begin{array}{l}\text { Top of } \\
\text { Sample } \\
\text { (ft) }\end{array}$ & $\begin{array}{l}\text { Eottom of } \\
\text { Sample } \\
\text { (H) }\end{array}$ & $\begin{array}{l}\text { Fhysical } \\
\text { Analysis }\end{array}$ & $\begin{array}{l}\text { Chemicsl } \\
\text { Analysis }\end{array}$ & $\begin{array}{l}\text { Sample } \\
\text { Archived }\end{array}$ & Comments \\
\hline \multicolumn{8}{|c|}{$\begin{array}{l}307 \text { Retention Basin } \\
\text { Borehole: } 307 F 8-2,399-3-14\end{array}$} \\
\hline E014T0 & $11111 / 91$ & 0.0 & 2.0 & \multirow{23}{*}{$x$} & $\bar{x}$ & \multirow{23}{*}{$x$} & \multirow{8}{*}{$\begin{array}{l}\text { Duplicate } \\
\text { Duplicate. }\end{array}$} \\
\hline E014TI & $11 / 11 / 91$ & 0.0 & 2.0 & & $x$ & & \\
\hline$E 015 Z 2$ & $11 / 91 / 91$ & 0.0 & 2.0 & & & & \\
\hline $8014 T 2$ & $11 / 11 / 91$ & 5.0 & 7.0 & & $x$ & & \\
\hline $8014 T 3$ & $11 / 11 / 91$ & 5.0 & 7.0 & & $x$ & & \\
\hline $8014 T 4$ & 11/12/91 & 7.0 & 9.0 & & $x$ & & \\
\hline $8015 Z 3$ & $11 / 11 / 91$ & 7.0 & 9.0 & & & & \\
\hline B014T5 & $11 / 12 / 91$ & 9.0 & 11.0 & & $x$ & & \\
\hline$B 014 P 1$ & $11 / 13 / 91$ & 11.0 & 13.0 & & $x$ & & \multirow{15}{*}{$\begin{array}{l}\text { Field blank } \\
\text { Field blank }\end{array}$} \\
\hline E014P2 & $11 / 14 / 91$ & 16.5 & 18.5 & & $x$ & & \\
\hline E014P3 & $11 / 14 / 91$ & 20.0 & 22.0 & & $x$ & & \\
\hline Bo1C83 & $11 / 15 / 91$ & 25.0 & 27.0 & & $x$ & & \\
\hline 801524 & $11 / 15 / 91$ & 25.0 & 27.0 & & & & \\
\hline $801 C 84$ & $11 / 15 / 91$ & 30.0 & 32.0 & & $x$ & & \\
\hline e01C85 & $11 / 18 / 91$ & & & & $x$ & & \\
\hline E01C86 & $11 / 18 / 91$ & & & & $x$ & & \\
\hline 801687 & $111 / 18 / 91$ & 35.0 & 37.0 & & $x$ & & \\
\hline $801 C 88$ & $11 / 18 / 91$ & & & & $x$ & & \\
\hline E01C89 & $11 / 18 / 91$ & 40.0 & 42.0 & & $x$ & & \\
\hline E01CCO & $11 / 18 / 91$ & 45.0 & 47.0 & & $x$ & & \\
\hline $\mathrm{EO}^{\circ \mathrm{CC}_{1}}{ }^{\circ}$ & $11 / 18 / 91$ & 47.0 & 49.0 & & $x$ & & \\
\hline$E 01 C^{\circ} 2^{*}$ & $11 / 20 / 91$ & 54.0 & 56.0 & & $x$ & & \\
\hline E014W9 & & & & & $x$ & & \\
\hline
\end{tabular}


WHC-SD-EN-TI-279, Rev. 0

Table 4. 307 Trenches Boreholes 399-3-15, 399-3-16 and 399-3-17. (Sheet 1 of 3)

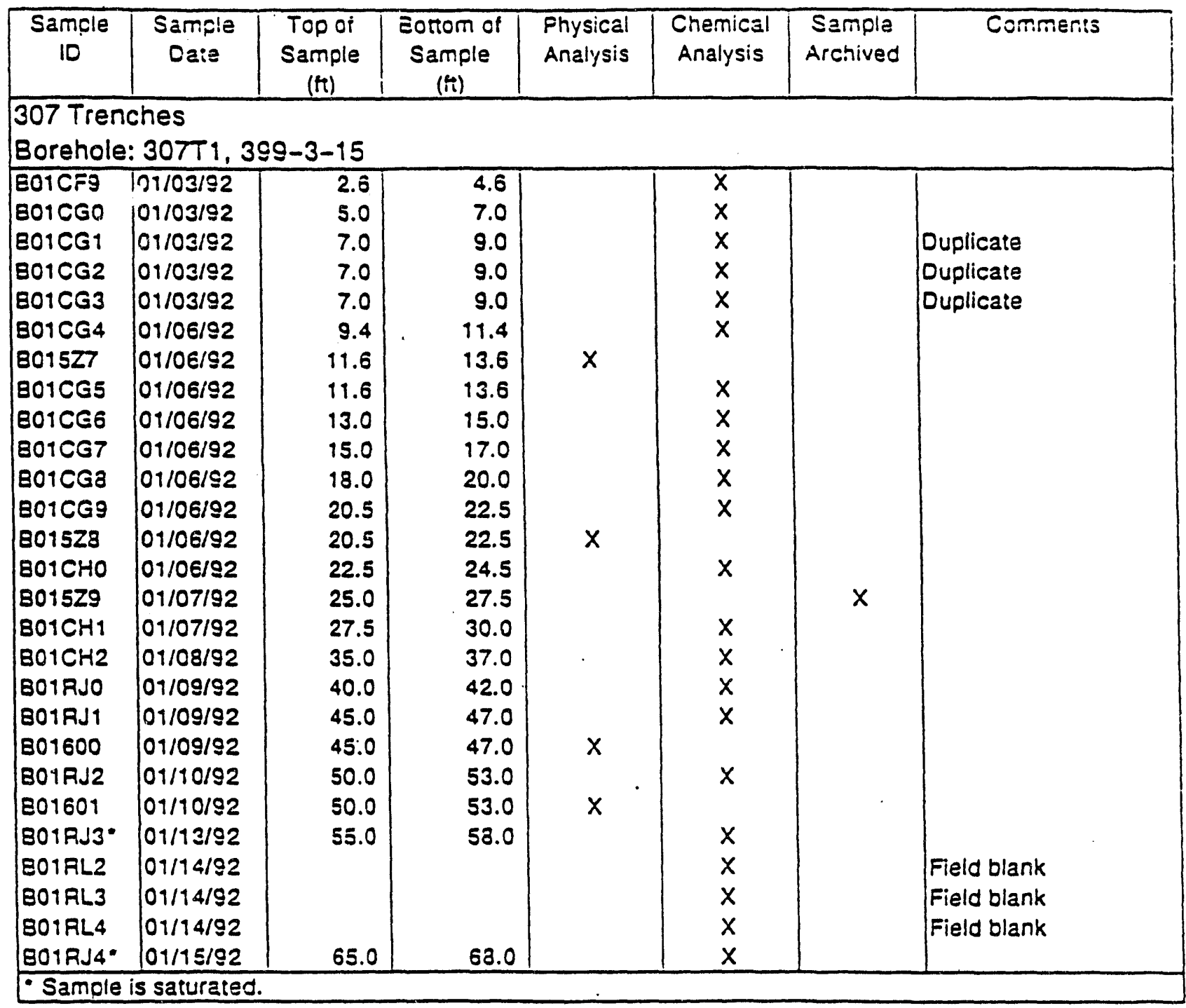


Table 4. 307 Trenches Boreholes 399-3-15, 399-3-16 and 399-3-17. (Sheet 2 of 3)

\begin{tabular}{|c|c|c|c|c|c|c|c|}
\hline $\begin{array}{c}\text { Sample } \\
10\end{array}$ & $\begin{array}{c}\text { Sample } \\
\text { Date }\end{array}$ & $\begin{array}{l}\text { Top of } \\
\text { Sample } \\
\text { (ft) }\end{array}$ & $\begin{array}{c}\text { Eotrcm of } \\
\text { Sample } \\
\text { (fi) }\end{array}$ & $\begin{array}{l}\text { Fhysical } \\
\text { Analysis }\end{array}$ & $\begin{array}{c}\text { Chemical } \\
\text { Analysis }\end{array}$ & $\begin{array}{l}\text { Sample } \\
\text { Archived }\end{array}$ & Comments \\
\hline \multicolumn{8}{|c|}{307 Trenches } \\
\hline \multicolumn{8}{|c|}{ Borehole: $30772.399-3-16$} \\
\hline EO1CC3 & $12 / 02 / 91$ & 0.0 & 3.0 & & $\bar{x}$ & & Duplicate \\
\hline BO1CC4 & $12 / 02 / 91$ & 0.0 & 3.0 & & $x$ & & Duplicate \\
\hline E01CC5 & $12 / 02 / 91$ & 2.9 & 4.9 & & $x$ & & \\
\hline $801 C \mathrm{C6}$ & $12 / 02 / 91$ & 4.9 & 6.0 & & $x$ & & \\
\hline Bo1CC7 & $12 / 04 / 91$ & 8.3 & 10.3 & & $\mathrm{x}$ & & \\
\hline E01CD4 & $12 / 04 / 91$ & 10.4 & 12.4 & & $x$ & & \\
\hline 801605 & $12 / 04 / 91$ & 12.3 & 14.3 & & $x$ & & \\
\hline 801CD6 & $12 / 04 / 91$ & 14.3 & 16.3 & & $x$ & & Split to S01CD7 \\
\hline 801607 & $12 / 04 / 91$ & 14.3 & 16.3 & & $x$ & & \\
\hline $801 C 08$ & $12 / 05191$ & 16.4 & 18.4 & & $x$ & & \\
\hline 801525 & $12 / 05 / 91$ & 16.4 & 18.4 & $x$ & & & \\
\hline 801609 & $12 / 05 / 91$ & 18.4 & 20.4 & & $x$ & & \\
\hline Bo1CFO & $12 / 05 / 91$ & 20.4 & 22.4 & & $x$ & & \\
\hline BO1CF1 & $12 / 05 / 91$ & 22.0 & 24.0 & & $x$ & & \\
\hline Bo1CF2 & $12 / 06 / 91$ & 24.0 & 26.0 & & $x$ & & \\
\hline B01CF3 & $12 / 06 / 91$ & 28.7 & 30.7 & & $x$ & & \\
\hline 801526 & $12 / 06 / 91$ & 28.7 & 30.7 & $x$ & & & \\
\hline $801 C F 4$ & $12 / 09 / 91$ & 34.0 & 36.0 & & $x$ & & \\
\hline Bo1CF5 & $12 / 09 / 91$ & 39.0 & 49.0 & & $x$ & & \\
\hline B01CF6 & $12 / 10 / 91$ & 45.0 & 47.0 & & $x$ & & \\
\hline B01CC9 & $12 / 11 / 91$ & & & & $x$ & & Field blank \\
\hline 801600 & $12 / 11 / 91$ & & & & $x$ & & Field blank \\
\hline B01CD1 & $12 / 11 / 91$ & & & & $x$ & & Field blank \\
\hline $801 \mathrm{CF}^{\circ}$ & $12 / 11 / 91$ & 56.0 & 58.0 & & $x$ & & \\
\hline B01CF8. & $12 / 11 / 91$ & 58.0 & 60.0 & & $x$ & & \\
\hline
\end{tabular}


WHC-SD-EN-TI-279, Rev. 0

Table 4. 307 Trenches Boreholes 399-3-15, 399-3-16 and 399-3-17. (Sheet 3 of 3)

\begin{tabular}{|c|c|c|c|c|c|c|c|}
\hline $\begin{array}{c}\text { Sample } \\
10\end{array}$ & $\begin{array}{l}\text { Sample } \\
\text { Date }\end{array}$ & $\begin{array}{c}\text { Top of } \\
\text { Sample } \\
\text { (ft) }\end{array}$ & $\begin{array}{c}\text { Eottom of } \\
\text { Sample } \\
\text { (4i) }\end{array}$ & $\begin{array}{l}\text { Physical } \\
\text { Analysis }\end{array}$ & $\begin{array}{l}\text { Chemical } \\
\text { Analysis }\end{array}$ & $\begin{array}{l}\text { Sample } \\
\text { Arshived }\end{array}$ & Comments \\
\hline \multicolumn{8}{|c|}{$\begin{array}{l}307 \text { Trenches } \\
\text { Borehole: } 30 \pi T 3,399-3-17\end{array}$} \\
\hline 801RJ5 & $01 / 20 / 92$ & 6.4 & 8.4 & & $\bar{x}$ & & \\
\hline 801RJ6 & 01/20/92 & 6.4 & 8.4 & & $x$ & & Solit to 801RJJ5 \\
\hline 801R.J7 & 01/20/92 & 8.4 & 10.4 & & $x$ & & Duplicate \\
\hline E01RJ8 & 01/20/92 & 8.4 & 10.4 & & $x$ & & Duplicate \\
\hline B01RJ9 & 01/21/92 & 11.6 & 13.6 & & $x$ & & \\
\hline 801602 & $01 / 21 / 92$ & 11.6 & 13.6 & $x$ & & & \\
\hline BO1AKO & 01/21/92 & 13.5 & 15.5 & & $x$ & & \\
\hline BO1AKI & |01/21/82 & 15.7 & 17.7 & & $x$ & & \\
\hline B01RK2 & |01/22/92 & 17.9 & 19.9 & & $x$ & & \\
\hline 8017K3 & 01/22/92 & 20.0 & 22.0 & & $x$ & & \\
\hline BO1AK4 & $01 / 22 / 92$ & 22.0 & 24.0 & & $x$ & & \\
\hline B01RK5 & |01/22/92 & 25.3 & 27.3 & & $x$ & & \\
\hline BO1FK6 & $01 / 23 / 32$ & 31.0 & 33.0 & & $x$ & & \\
\hline$E 01603$ & $01 / 23 / 92$ & 31.0 & 33.0 & $x$ & & & \\
\hline BOIRK7 & 01/23/92 & 36.0 & 38.0 & & $x$ & & \\
\hline 801RK9" & $01 / 24 / 92$ & 50.0 & 52.0 & & $x$ & & \\
\hline E01RK8* & 01/27/92 & 63.0 & 65.0 & & $x$ & & \\
\hline B01604" & 01/27/92 & 63.0 & 65.0 & $x$ & & & \\
\hline B01RL6 & 01/27/92 & & & & $x$ & & Field blank \\
\hline BO1RL7 & 01/27/92 & & & & $x$ & & Field blank \\
\hline EOIRLB & $01 / 28 / 92$ & & & & $x$ & & Field blank \\
\hline
\end{tabular}


WHC-SD-EN-TI-279, Rev. 0

\section{APPENDDX A \\ SOIL ANALYTICAL RESULTS}




\section{DATA QUALIFIERS}

$\mathrm{U}$ The material was analyzed for but was not detected. The associated value is the sample quantitation limit (SQL).

J The associated value is an estimated quantity.

UJ The material was analyzed for but was not detected. The SQL is an estimated quantity.

R The data are unusable.

N/R Not reported and/or not requested.

D The value determined is the result of a dilution.

$X \quad$ The value was entered by hand.

B For organics analyses this indicates compound was detected in the blank. For inorganics analyses this indicates the detected concentration is between the instrument detection limit and the contract required quantitation limit.

N Spiked sample recovery not within control limits.

Note: Qualifiers may be combined (e.g., $\mathrm{N}$-the value is estimated and the spiked sample recovery was not within control limits) 
Location 307 RB-1, 399-3-13

\begin{tabular}{|c|c|c|c|c|c|c|c|c|c|c|c|c|c|}
\hline \multirow[t]{2}{*}{ Parameter } & \multirow{2}{*}{$\begin{array}{l}\text { Samp: } \\
\text { Depth }\end{array}$} & \multicolumn{2}{|l|}{$\begin{array}{r}01409 \\
2.00 \\
\end{array}$} & \multicolumn{2}{|c|}{$\begin{array}{c}\text { BO14Q9RE } \\
2.00\end{array}$} & \multicolumn{2}{|l|}{$\begin{array}{r}\text { BO14R0 } \\
7.50\end{array}$} & \multicolumn{2}{|c|}{$\begin{array}{c}\text { B014RORE } \\
7.50\end{array}$} & \multicolumn{2}{|l|}{$\begin{array}{r}8014 R 2 \\
i 0.50\end{array}$} & \multicolumn{2}{|l|}{$\begin{array}{r}801423 \\
13.00\end{array}$} \\
\hline & & Result & 0 & Result & a & Result & a & Result & a & Result & a & Result & 0 \\
\hline 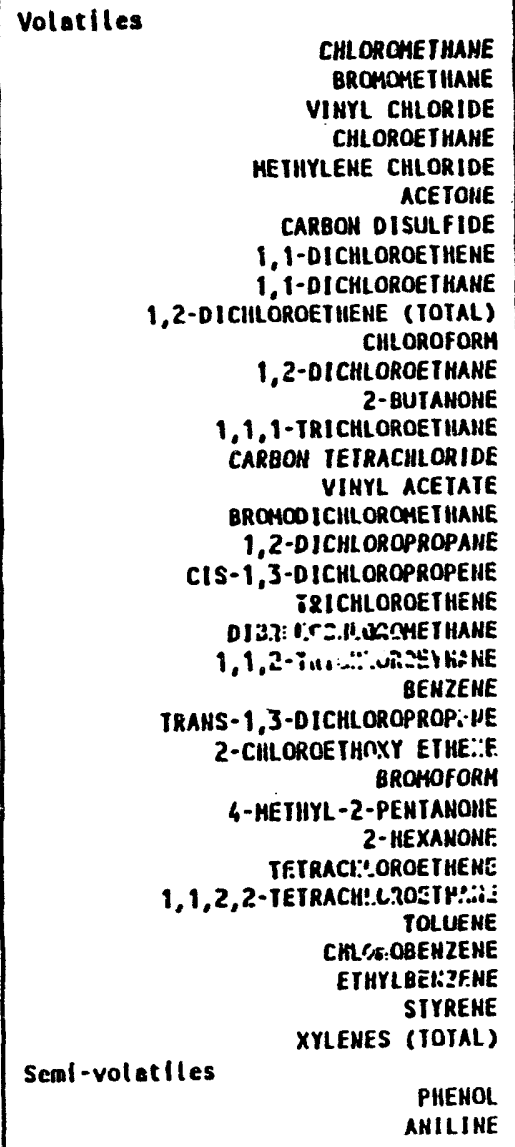 & 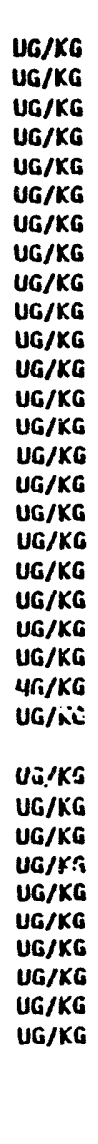 & $\begin{array}{r}10.000 \\
10.000 \\
10.000 \\
10.000 \\
7.000 \\
34.000 \\
13.000 \\
5.000 \\
5.000 \\
5.000 \\
1.000 \\
5.000 \\
10.000 \\
5.000 \\
5.000 \\
10.000 \\
5.000 \\
5.000 \\
5.000 \\
2.000 \\
5.000 \\
5.000 \\
5.000 \\
5.000 \\
N / R \\
5.000 \\
10.000 \\
1 C .000 \\
5.000 \\
5.000 \\
4.000 \\
5.000 \\
5.000 \\
5.000 \\
5.000 \\
\\
H / R \\
H / R\end{array}$ & $\begin{array}{c}u \\
u \\
u \\
u \\
u \\
u \\
u \\
u \\
u \\
u \\
u \\
u \\
u \\
u \\
u \\
u \\
u \\
u \\
u \\
J u \\
u \\
u \\
u \\
u \\
u \\
u \\
u \\
u \\
u \\
u \\
J u \\
u \\
u \\
u \\
v\end{array}$ & $\begin{array}{r}10.000 \\
10.000 \\
10.000 \\
10.000 \\
8.000 \\
12.000 \\
8.000 \\
5.000 \\
5.000 \\
5.000 \\
3.000 \\
5.000 \\
10.000 \\
5.000 \\
5.000 \\
10.000 \\
5.000 \\
5.000 \\
5.000 \\
5.000 \\
5.000 \\
5.000 \\
5.000 \\
5.000 \\
H / R \\
5.000 \\
10.000 \\
10.000 \\
5.000 \\
5.000 \\
i .000 \\
5.000 \\
5.000 \\
5.000 \\
5.000 \\
\\
H / R \\
H / R\end{array}$ & $\begin{array}{c}u \\
u \\
u \\
u \\
u \\
u \\
u \\
u \\
u \\
u \\
J u \\
u \\
u \\
u \\
u \\
u \\
u \\
u \\
u \\
u \\
u \\
u \\
u \\
u \\
u \\
u \\
u \\
u \\
u \\
J u \\
u \\
u \\
u \\
u\end{array}$ & $\begin{array}{r}11.000 \\
11.000 \\
11.000 \\
11.000 \\
6.000 \\
56.000 \\
5.000 \\
5.000 \\
5.000 \\
5.000 \\
5.000 \\
5.000 \\
11.000 \\
5.000 \\
5.000 \\
11.000 \\
5.000 \\
5.000 \\
5.000 \\
5.000 \\
5.000 \\
5.000 \\
5.000 \\
5.000 \\
H / R \\
5.060 \\
11.000 \\
11.000 \\
5.000 \\
5.000 \\
5.000 \\
5.600 \\
5.000 \\
5.000 \\
5.000 \\
H / R \\
H / R\end{array}$ & $\begin{array}{l}u \\
u \\
u \\
u \\
u \\
u \\
u \\
u \\
u \\
u \\
u \\
u \\
u \\
u \\
u \\
u \\
u \\
u \\
u \\
u \\
u \\
u \\
u \\
u \\
u \\
u \\
u \\
u \\
u \\
u \\
u \\
u \\
u \\
u \\
u\end{array}$ & $\begin{array}{r}11.000 \\
11.000 \\
11.000 \\
11.000 \\
5.000 \\
12.000 \\
5.000 \\
5.000 \\
5.000 \\
5.000 \\
5.000 \\
5.000 \\
11.000 \\
5.000 \\
5.000 \\
11.000 \\
5.000 \\
5.000 \\
5.000 \\
5.000 \\
5.000 \\
5.000 \\
5.000 \\
5.000 \\
H / R \\
5.000 \\
11.006 \\
11.000 \\
5.000 \\
5.000 \\
5.000 \\
5.000 \\
5.000 \\
5.000 \\
5.000 \\
H / R \\
H / R\end{array}$ & $\begin{array}{c}u \\
u \\
u \\
u \\
u \\
u \\
u \\
u \\
u \\
u \\
u \\
u \\
u \\
u \\
u \\
u \\
u \\
u \\
u \\
u \\
u \\
u \\
u \\
u \\
u \\
u \\
u \\
v \\
u \\
u \\
u \\
u \\
u \\
u\end{array}$ & $\begin{array}{r}11.000 \\
11.000 \\
11.000 \\
11.000 \\
5.000 \\
22.000 \\
5.000 \\
5.000 \\
5.000 \\
5.000 \\
2.000 \\
5.000 \\
11.000 \\
5.000 \\
5.000 \\
11.000 \\
5.060 \\
5.000 \\
5.000 \\
5.000 \\
5.000 \\
5.000 \\
5.000 \\
5.000 \\
H / R \\
5.000 \\
11.000 \\
11.000 \\
5.000 \\
5.000 \\
5.000 \\
5.000 \\
5.000 \\
5.000 \\
5.000 \\
H / R \\
11 / k\end{array}$ & $\begin{array}{l}u \\
u \\
u \\
u \\
u \\
u \\
u \\
u \\
u \\
u \\
u \\
u \\
u \\
u \\
u \\
u \\
u \\
u \\
u \\
u \\
u \\
u \\
u \\
u \\
u \\
u \\
u \\
u \\
u \\
u \\
u \\
u \\
u \\
u \\
u\end{array}$ & $\begin{array}{r}11.000 \\
11.000 \\
11.000 \\
11.000 \\
5.000 \\
40.000 \\
6.000 \\
5.000 \\
5.000 \\
5.000 \\
5.000 \\
5.000 \\
11.000 \\
5.000 \\
5.000 \\
11.000 \\
5.000 \\
5.000 \\
5.000 \\
5.000 \\
5.000 \\
5.000 \\
5.000 \\
5.000 \\
1 / R \\
5.000 \\
11.000 \\
11.000 \\
5.000 \\
5.000 \\
5.000 \\
5.000 \\
5.000 \\
5.000 \\
5.000 \\
H / R \\
11 / R\end{array}$ & $\begin{array}{l}u \\
u \\
u \\
u \\
u \\
u \\
u \\
u \\
u \\
u \\
u \\
u \\
u \\
u \\
u \\
u \\
u \\
u \\
u \\
u \\
u \\
u \\
u \\
u \\
u \\
u \\
u \\
u \\
u \\
u \\
u \\
u \\
u \\
u\end{array}$ \\
\hline
\end{tabular}


WHC-SD-EN-TI-279, Rev. 0

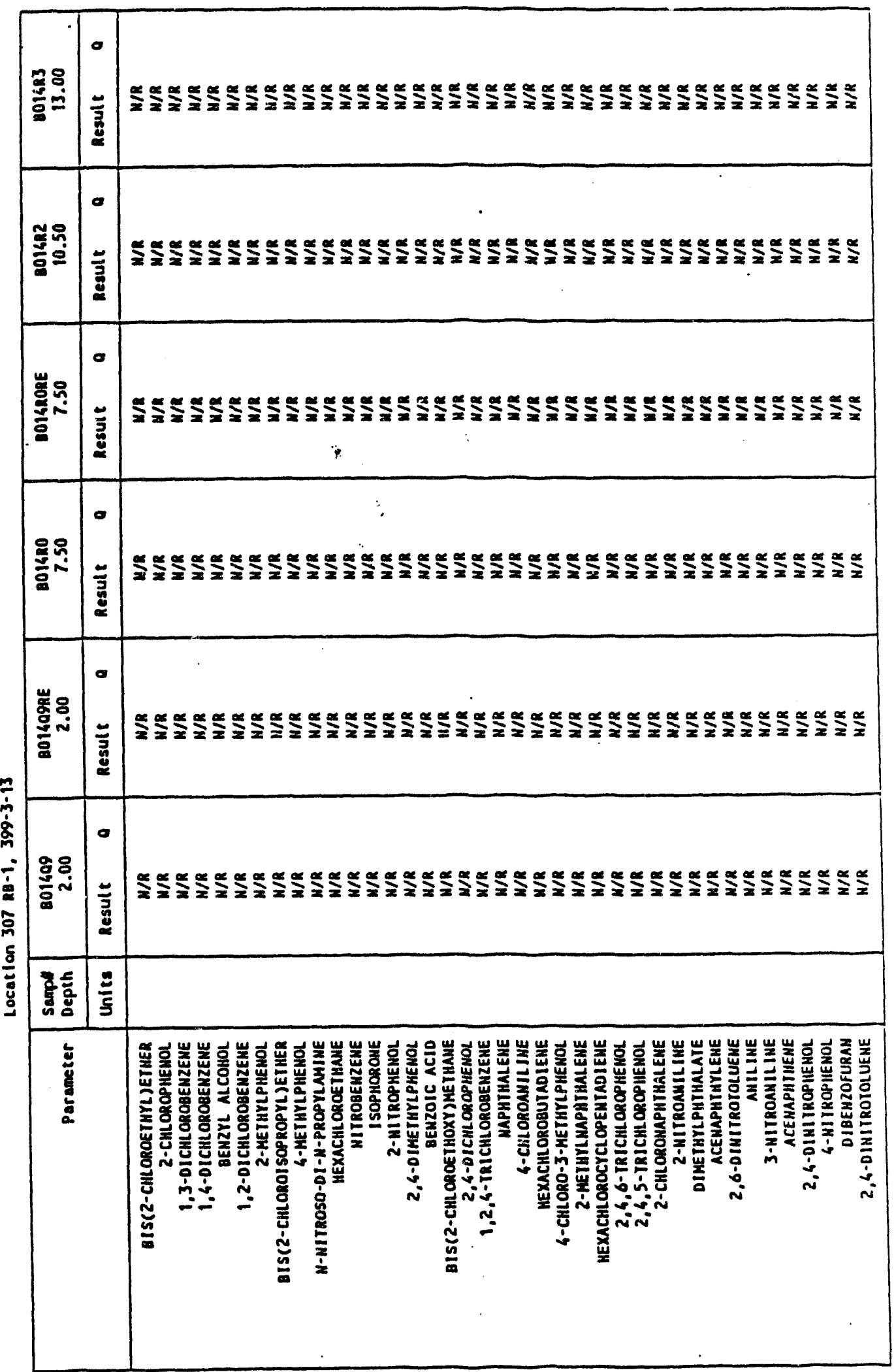


Location 307 88-1, 399-3-13

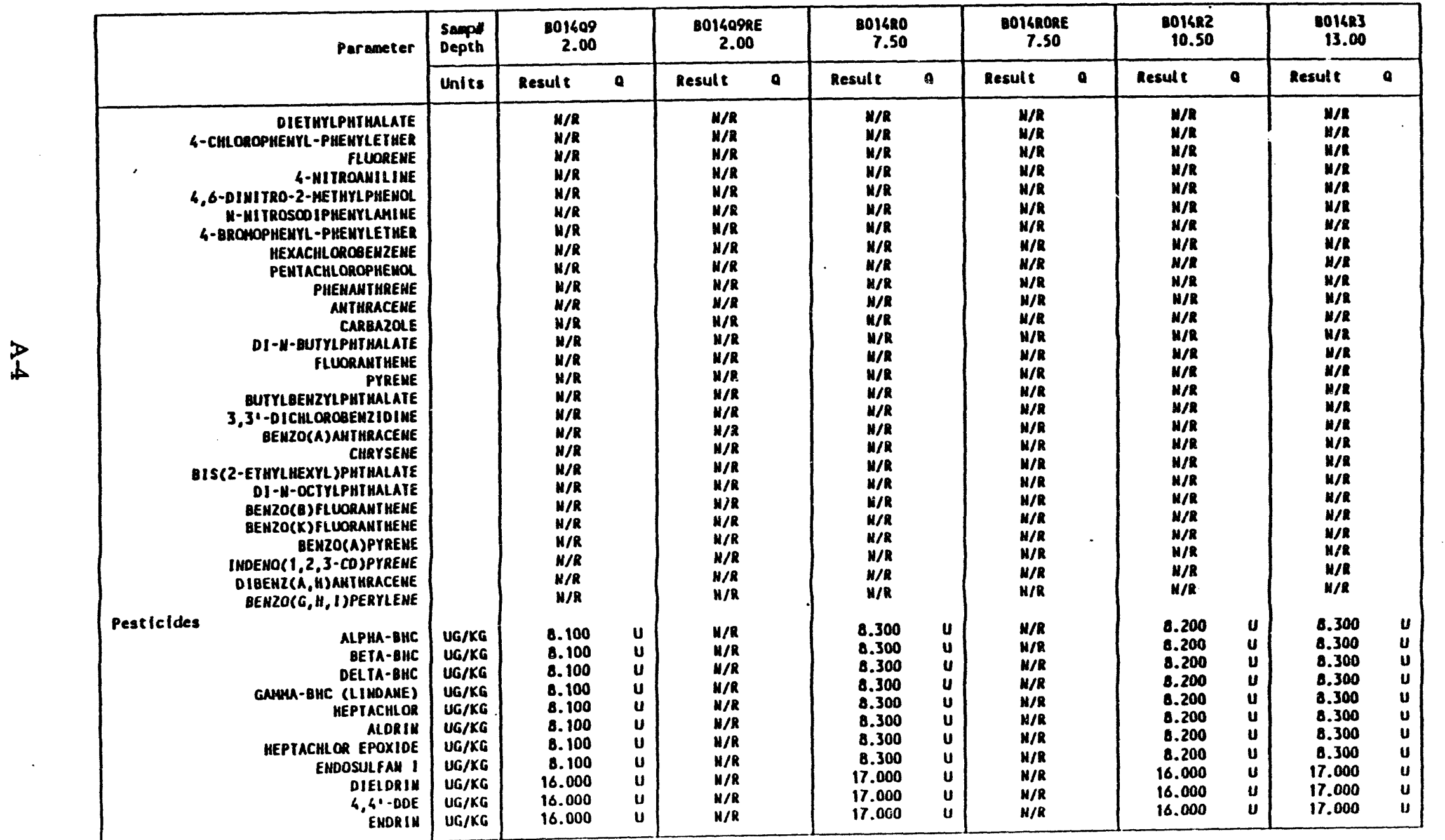




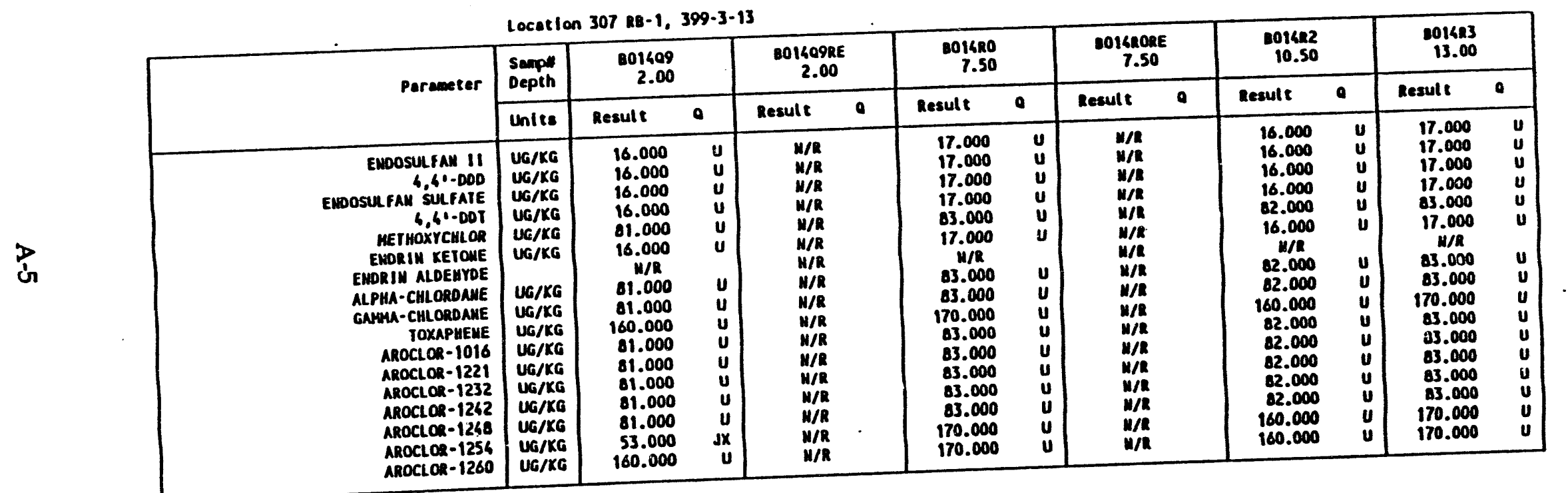


Location 307 RB-1, 399-3-13

\begin{tabular}{|c|c|c|c|c|c|c|c|c|c|c|c|c|c|}
\hline \multirow[t]{2}{*}{ Parancter } & \multirow{2}{*}{$\begin{array}{l}\text { Semp" } \\
\text { Depth }\end{array}$} & \multicolumn{2}{|l|}{$\begin{array}{r}8014 R 6 \\
17.00\end{array}$} & \multicolumn{2}{|l|}{$\begin{array}{r}8016 R 5 \\
23.00\end{array}$} & \multicolumn{2}{|l|}{$\begin{array}{r}8014 R 6 \\
27.00\end{array}$} & \multicolumn{2}{|l|}{$\begin{array}{r}801488 \\
32.00\end{array}$} & \multicolumn{2}{|l|}{$\begin{array}{r}\text { O016Ri } \\
37.00\end{array}$} & \multicolumn{2}{|l|}{$\begin{array}{r}801450 \\
46.00\end{array}$} \\
\hline & & Result & 0 & Result & a & Result & a & Result & $a$ & Result & $a$ & Result & a \\
\hline 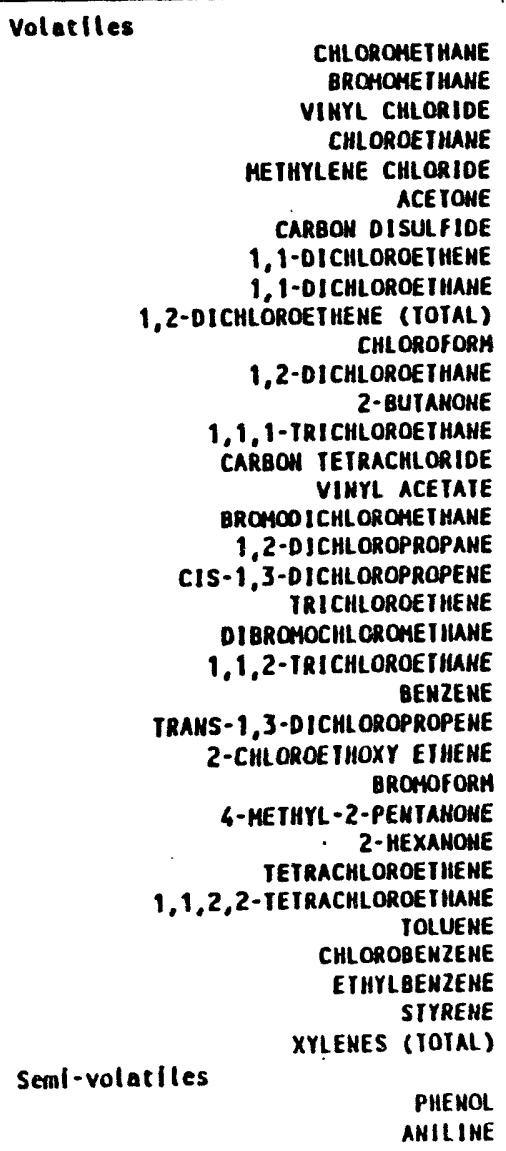 & $\begin{array}{l}U G / K G \\
U G / K G \\
U G / K G \\
U G / K G \\
U G / K G \\
U G / K G \\
U G / K G \\
U G / K G \\
U G / K G \\
U G / K G \\
U G / K G \\
U G / K G \\
U G / K G \\
U G / K G \\
U G / K G \\
U G / K G \\
U G / K G \\
U G / K G \\
U G / K G \\
U G / K G \\
U G / K G \\
U G / K G \\
U G / K G \\
U G / K G \\
U G / K G \\
U G / K G \\
U G / K G \\
U G / K G \\
U G / K G \\
U G / K G \\
U G / K G \\
U G / K G \\
U G / K G \\
U G / K G\end{array}$ & $\begin{array}{r}11.000 \\
11.000 \\
11.000 \\
11.000 \\
5.000 \\
30.000 \\
6.000 \\
5.000 \\
5.000 \\
5.000 \\
5.000 \\
5.000 \\
11.000 \\
5.000 \\
5.000 \\
11.000 \\
5.000 \\
5.000 \\
5.000 \\
5.000 \\
5.000 \\
5.000 \\
2.000 \\
5.000 \\
H / R \\
5.000 \\
11.000 \\
11.000 \\
5.000 \\
5.000 \\
2.000 \\
5.000 \\
5.000 \\
5.000 \\
5.000 \\
H / R \\
H / R\end{array}$ & $\begin{array}{c}\mathbf{u} \\
\mathbf{u} \\
\mathbf{u} \\
\mathbf{u} \\
\mathbf{u} \\
\mathbf{u} \\
\mathbf{u} \\
\mathbf{u} \\
\mathbf{u} \\
\mathbf{u} \\
\mathbf{u} \\
\mathbf{u} \\
\mathbf{u} \\
\mathbf{u} \\
\mathbf{u} \\
\mathbf{u} \\
\mathbf{u} \\
\mathbf{u} \\
\mathbf{u} \\
\mathbf{u} \\
\mathbf{u} \\
\mathbf{u} \\
\mathbf{J u} \\
\mathbf{u} \\
\mathbf{u} \\
\mathbf{u} \\
\mathbf{u} \\
\mathbf{u} \\
\mathbf{u} \\
\mathbf{u} \\
\mathbf{u} \\
\mathbf{u} \\
\mathbf{u} \\
\mathbf{u}\end{array}$ & $\begin{array}{r}11.000 \\
11.000 \\
11.000 \\
11.000 \\
5.000 \\
42.000 \\
5.000 \\
5.000 \\
5.000 \\
5.000 \\
5.000 \\
5.000 \\
11.000 \\
5.000 \\
5.000 \\
11.000 \\
5.000 \\
5.000 \\
5.000 \\
5.000 \\
5.000 \\
5.000 \\
3.000 \\
5.000 \\
11 / R \\
5.000 \\
11.000 \\
11.000 \\
5.000 \\
5.000 \\
4.000 \\
5.000 \\
5.000 \\
5.000 \\
5.000 \\
\\
11 / R \\
1 / 8\end{array}$ & 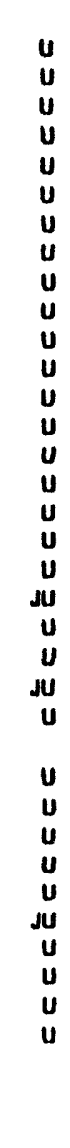 & $\begin{array}{r}11.000 \\
11.000 \\
11.000 \\
11.000 \\
6.000 \\
62.000 \\
4.000 \\
5.000 \\
5.000 \\
5.000 \\
5.000 \\
5.000 \\
11.000 \\
5.000 \\
5.000 \\
11.000 \\
5.000 \\
5.000 \\
5.000 \\
5.000 \\
5.000 \\
5.000 \\
5.000 \\
5.000 \\
1 \% / R \\
5.000 \\
11.000 \\
11.000 \\
5.000 \\
5.000 \\
2.000 \\
5.000 \\
5.000 \\
5.000 \\
5.000 \\
H / R \\
H / R\end{array}$ & 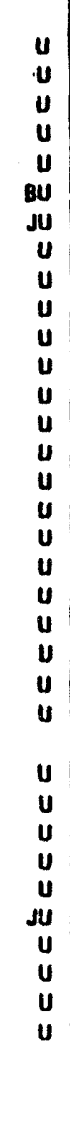 & $\begin{array}{r}11.000 \\
11.000 \\
11.000 \\
11.000 \\
10.000 \\
65.000 \\
4.000 \\
5.000 \\
5.000 \\
5.000 \\
2.000 \\
5.000 \\
11.000 \\
5.000 \\
5.000 \\
11.000 \\
5.000 \\
5.000 \\
5.000 \\
5.000 \\
5.000 \\
5.000 \\
5.000 \\
5.000 \\
11 / R \\
5.000 \\
11.000 \\
11.000 \\
5.000 \\
5.000 \\
1.000 \\
5.000 \\
5.000 \\
5.000 \\
5.000 \\
H / R \\
1 / R\end{array}$ & $\begin{array}{c}u \\
u \\
u \\
u \\
u \\
B u \\
u \\
u \\
u \\
u \\
J u \\
u \\
u \\
u \\
u \\
u \\
u \\
u \\
u \\
u \\
u \\
u \\
u \\
u \\
u \\
u \\
u \\
u \\
u \\
u \\
u \\
u \\
u \\
u\end{array}$ & $\begin{array}{r}11.000 \\
11.000 \\
11.000 \\
11.000 \\
9.000 \\
57.000 \\
6.000 \\
5.000 \\
5.000 \\
5.000 \\
5.000 \\
5.000 \\
11.000 \\
5.000 \\
5.000 \\
11.000 \\
5.000 \\
5.000 \\
5.000 \\
5.000 \\
5.000 \\
5.000 \\
5.000 \\
1.000 \\
11 / 8 \\
5.000 \\
11.000 \\
11.000 \\
5.000 \\
5.000 \\
1.000 \\
5.000 \\
5.000 \\
5.000 \\
5.000 \\
11 / R \\
11 / R\end{array}$ & $\begin{array}{l}u \\
u \\
u \\
u \\
u \\
u \\
u \\
u \\
u \\
u \\
u \\
u \\
u \\
u \\
u \\
u \\
u \\
u \\
u \\
u \\
u \\
u \\
u \\
u \\
u \\
u \\
u \\
u \\
u \\
u \\
u \\
u \\
u \\
u \\
u\end{array}$ & $\begin{array}{r}11.000 \\
11.000 \\
11.000 \\
11.000 \\
16.000 \\
70.000 \\
8.000 \\
5.000 \\
5.000 \\
5.000 \\
3.000 \\
5.000 \\
11.000 \\
5.000 \\
5.000 \\
11.000 \\
5.000 \\
5.000 \\
5.000 \\
5.000 \\
5.000 \\
5.000 \\
5.000 \\
5.000 \\
11 / 8 \\
5.000 \\
11.000 \\
11.000 \\
5.000 \\
5.000 \\
3.000 \\
5.000 \\
5.000 \\
5.000 \\
5.000 \\
11 / 8 \\
H / 8\end{array}$ & $\begin{array}{c}u \\
u \\
u \\
u \\
u \\
u u \\
u \\
u \\
u \\
u \\
u \\
u \\
u \\
u \\
u \\
u \\
u \\
u \\
u \\
u \\
u \\
u \\
u \\
u \\
u \\
u \\
u \\
u \\
u \\
u \\
u \\
u \\
u \\
u\end{array}$ \\
\hline
\end{tabular}


WHC-SD-EN-TI-279, Rev. 0

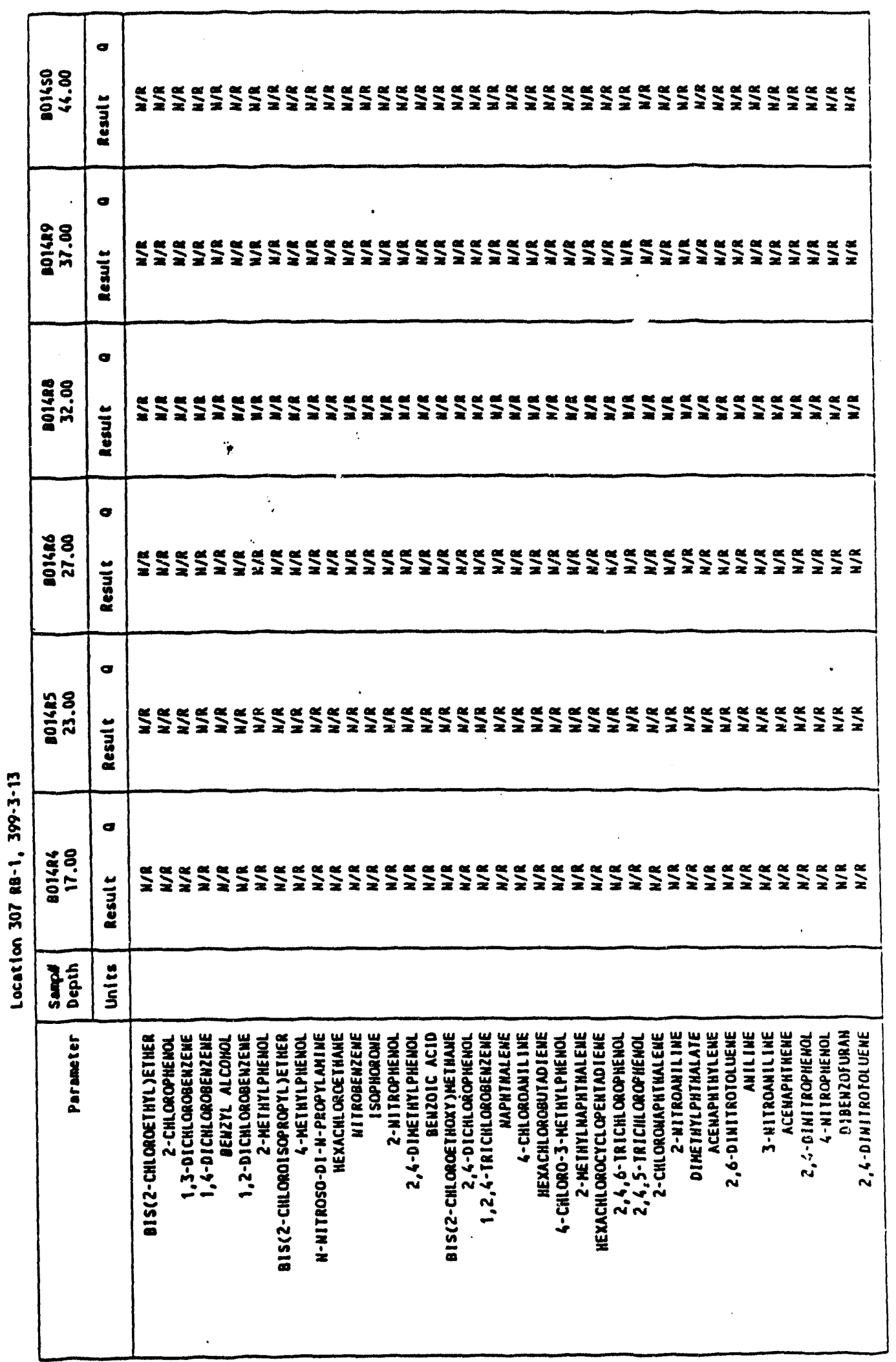




\begin{tabular}{|c|c|c|c|c|c|c|c|c|c|c|c|c|c|}
\hline \multirow[t]{2}{*}{ Parameter } & \multirow{2}{*}{ 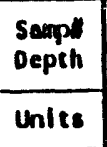 } & \multicolumn{2}{|l|}{$\begin{array}{l}8014 R 6 \\
17.00\end{array}$} & \multicolumn{2}{|l|}{$\begin{array}{r}101485 \\
23.00\end{array}$} & \multicolumn{2}{|l|}{$\begin{array}{r}8014866 \\
27.00\end{array}$} & \multicolumn{2}{|l|}{$\begin{array}{r}8014 R 8 \\
32.00\end{array}$} & \multicolumn{2}{|l|}{$\begin{array}{r}001629 \\
37.00\end{array}$} & \multicolumn{2}{|l|}{$\begin{array}{r}101450 \\
46.00\end{array}$} \\
\hline & & Result & $\mathbf{a}$ & Result & a & Result & $a$ & Result & a & Result & a & Result & a \\
\hline 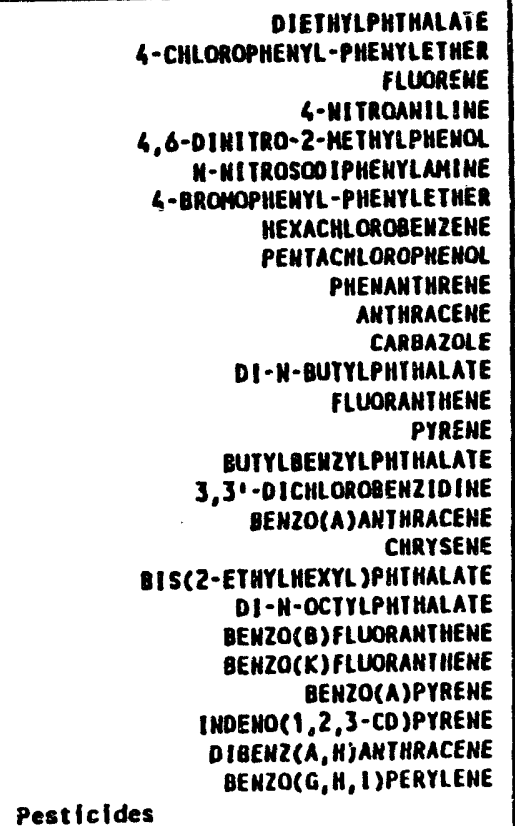 & $\cdot$ & 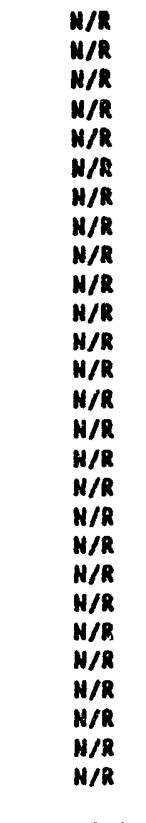 & & 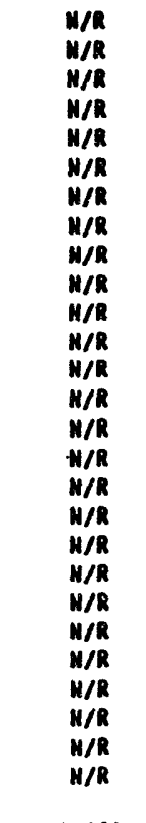 & $\cdot$ & 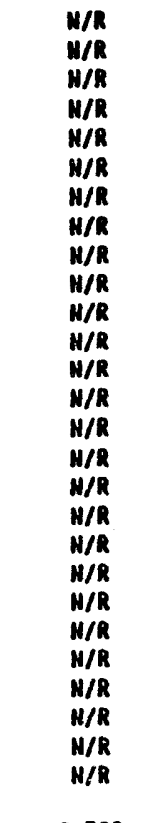 & & 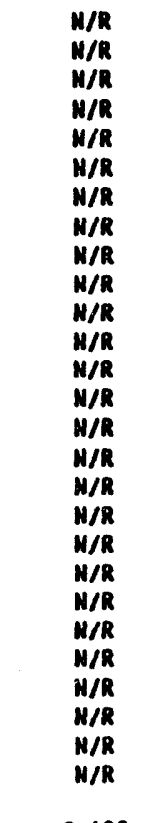 & & 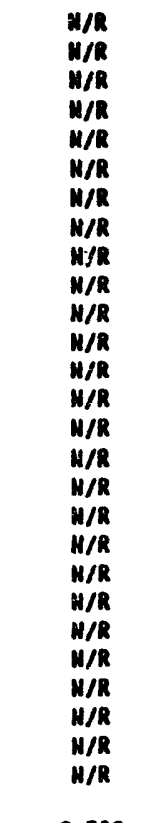 & ' & 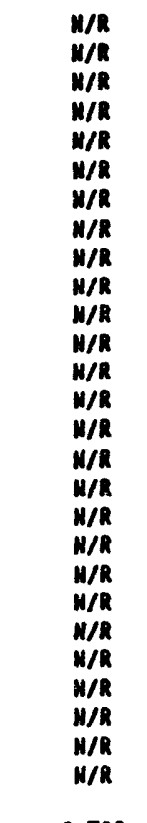 & \\
\hline 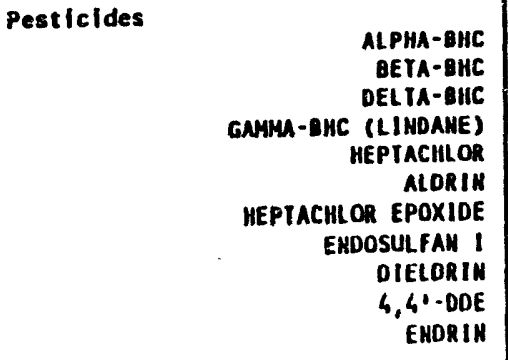 & $\begin{array}{l}U G / K G \\
U G / K G \\
U G / K G \\
U G / K G \\
U G / K G \\
U G / K G \\
U G / K G \\
U G / K G \\
U G / K G \\
U G / K G \\
U G / K G\end{array}$ & $\begin{array}{r}8.400 \\
8.400 \\
8.400 \\
8.400 \\
8.400 \\
8.400 \\
8.400 \\
8.400 \\
17.000 \\
17.000 \\
17.000\end{array}$ & $\begin{array}{l}\mathbf{u} \\
\mathbf{u} \\
\mathbf{u} \\
\mathbf{u} \\
\mathbf{u} \\
\mathbf{u} \\
\mathbf{u} \\
\mathbf{u} \\
\mathbf{u} \\
\mathbf{u} \\
\mathbf{u}\end{array}$ & $\begin{array}{l}8.400 \\
8.400 \\
8.400 \\
8.400 \\
8.400 \\
8.400 \\
8.400 \\
8.400 \\
17.000 \\
17.000 \\
17.000\end{array}$ & $\begin{array}{l}u \\
u \\
u \\
u \\
u \\
u \\
u \\
u \\
u \\
u \\
u \\
u\end{array}$ & $\begin{array}{l}8.300 \\
8.300 \\
8.300 \\
8.300 \\
6.300 \\
8.300 \\
8.300 \\
8.300 \\
17.000 \\
17.000 \\
17.000\end{array}$ & $\begin{array}{l}u \\
u \\
u \\
u \\
u \\
u \\
u \\
u \\
u \\
u \\
u\end{array}$ & $\begin{array}{r}8.400 \\
8.400 \\
8.400 \\
8.400 \\
8.400 \\
8.400 \\
8.400 \\
8.400 \\
17.000 \\
17.000 \\
17.000\end{array}$ & $\begin{array}{l}u \\
u \\
u \\
u \\
u \\
u \\
u \\
u \\
u \\
u \\
u\end{array}$ & $\begin{array}{r}8.500 \\
8.500 \\
8.500 \\
8.500 \\
8.500 \\
8.500 \\
8.500 \\
8.500 \\
17.000 \\
17.000 \\
17.000\end{array}$ & $\begin{array}{l}u \\
u \\
u \\
u \\
u \\
u \\
u \\
u \\
u \\
u \\
u\end{array}$ & $\begin{array}{r}8.700 \\
8.700 \\
8.700 \\
8.700 \\
8.700 \\
8.700 \\
8.700 \\
8.700 \\
17.000 \\
17.000 \\
17.000\end{array}$ & $\begin{array}{l}u \\
u \\
u \\
u \\
u \\
u \\
u \\
u \\
u \\
u \\
u\end{array}$ \\
\hline
\end{tabular}




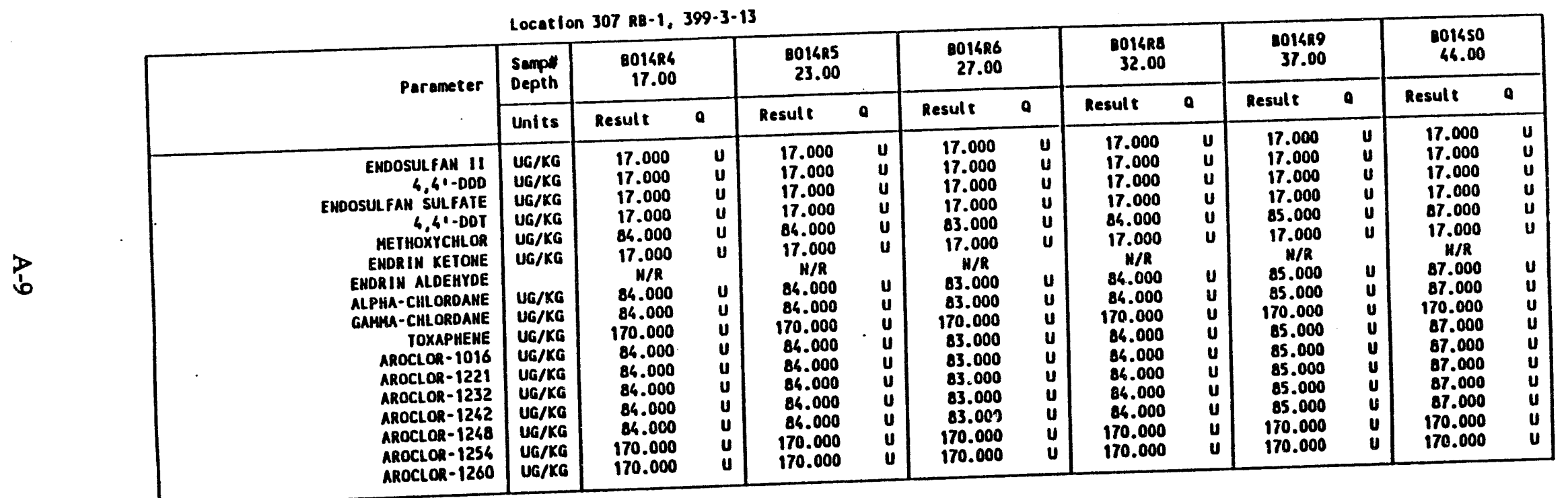


WHC-SD-EN-TI-279, Rev. 0

Location 307 88-1, 399-3-13

\begin{tabular}{|c|c|c|c|}
\hline \multirow[t]{2}{*}{ Parameter } & $\begin{array}{l}\text { Sempt } \\
\text { Depth }\end{array}$ & \multicolumn{2}{|l|}{$\begin{array}{r}801654 \\
67.00\end{array}$} \\
\hline & Units & Resule & $\mathbf{e}$ \\
\hline 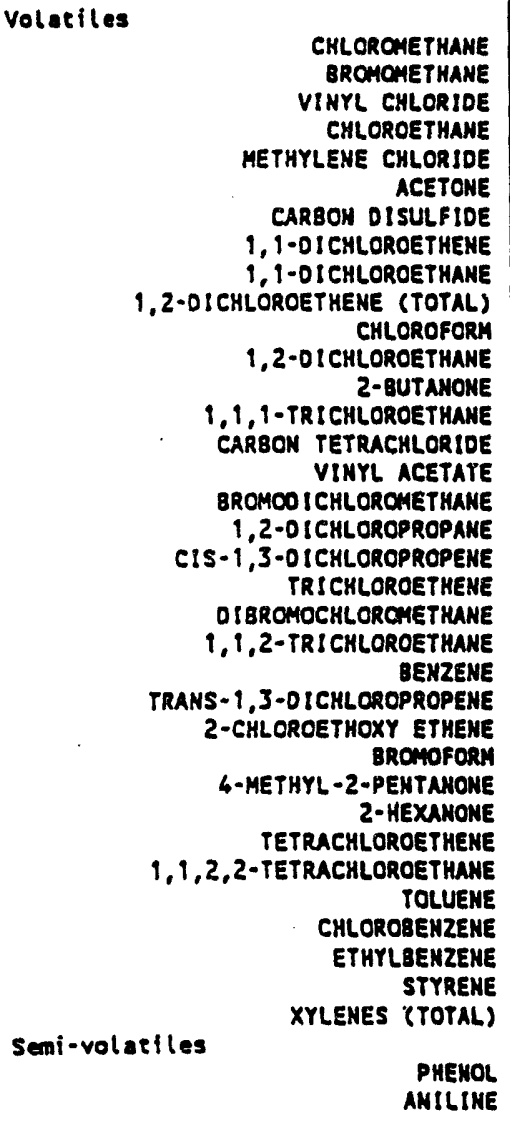 & $\begin{array}{l}\text { UG/KG } \\
\text { UG/KG } \\
\text { UG/KG } \\
\text { UG/KG } \\
\text { UG/KG } \\
\text { UG/KG } \\
\text { UG/KG } \\
\text { UG/KG } \\
\text { UG/KG } \\
\text { UG/KG } \\
\text { UG/KG } \\
\text { UG/KG } \\
\text { UG/KG } \\
\text { UG/KG } \\
\text { UG/KG } \\
\text { UG/KG } \\
\text { UG/KG } \\
\text { UG/KG } \\
\text { UG/KG } \\
\text { UG/KG } \\
\text { UG/KG } \\
\text { UG/KG } \\
\text { UG/KG } \\
\text { UG/KG } \\
\text { UG/KG } \\
\text { UG/KG } \\
\text { UG/KG } \\
\text { UG/KG } \\
\text { UG/KG } \\
\text { UG/KG } \\
\text { UG/KG } \\
\text { UG/KG } \\
\text { UG/KG } \\
\text { UG/KG }\end{array}$ & $\begin{array}{c}11.000 \\
11.000 \\
11.000 \\
11.000 \\
13.000 \\
66.000 \\
5.000 \\
6.000 \\
6.000 \\
6.000 \\
2.000 \\
6.000 \\
11.000 \\
6.000 \\
6.000 \\
11.000 \\
6.000 \\
6.000 \\
6.000 \\
6.000 \\
6.000 \\
6.000 \\
6.000 \\
6.000 \\
11 / R \\
6.000 \\
5.000 \\
11.000 \\
6.000 \\
6.000 \\
6.000 \\
6.000 \\
6.000 \\
6.000 \\
6.000 \\
\\
11 / R \\
11 / R\end{array}$ & 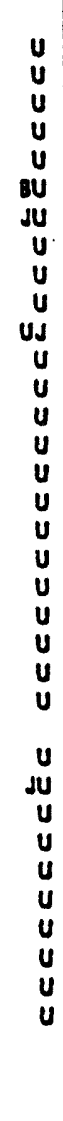 \\
\hline
\end{tabular}


WHC-SD-EN-TI-279, Rev. 0

Location 307 RB-1, 399-3-13

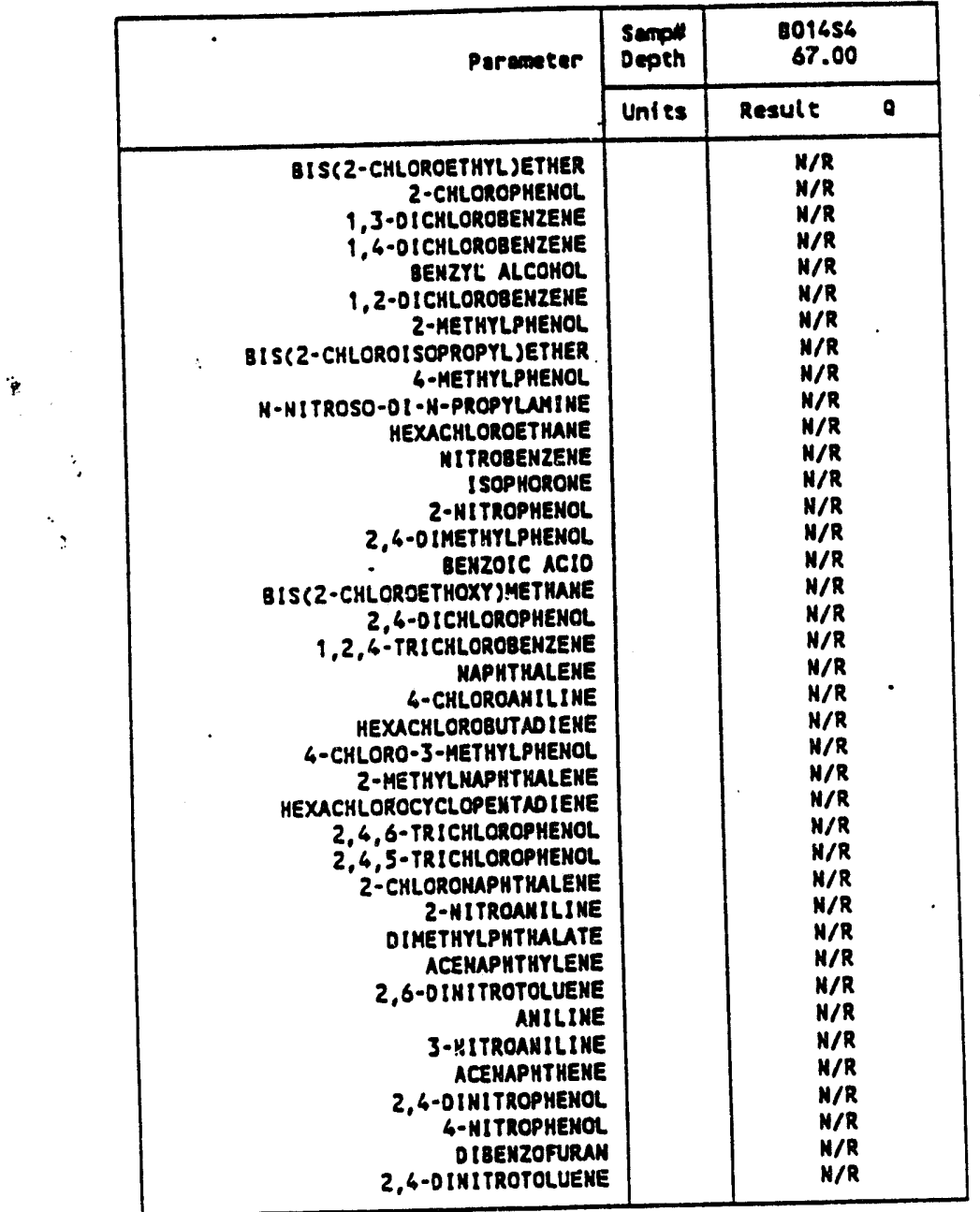


WHC-SD-EN-TI-279, Rev. 0

Location 307 RB-1, 399-3-13

\begin{tabular}{|c|c|c|c|}
\hline \multirow[t]{2}{*}{ Paraseter } & \multirow{2}{*}{$\frac{\begin{array}{l}\text { Sampil } \\
\text { Depth }\end{array}}{\text { Units }}$} & \multicolumn{2}{|l|}{$\begin{array}{r}801456 \\
67.00\end{array}$} \\
\hline & & Resule & 0 \\
\hline 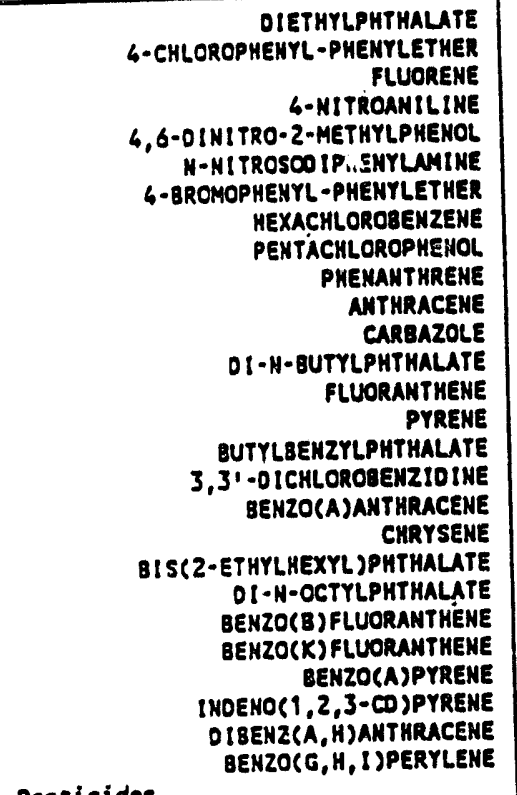 & & $\begin{array}{l}N / R \\
N / R \\
N / R \\
N / R \\
N / R \\
N / R \\
N / R \\
N / R \\
N / R \\
N / R \\
N / R \\
N / R \\
N / R \\
N / R \\
N / R \\
N / R \\
N / R \\
N / R \\
N / R \\
N / R \\
N / R \\
H / R \\
N / R \\
N / R \\
H / R \\
N / R \\
N / R \\
N / R\end{array}$ & \\
\hline $\begin{array}{r}\text { ALPHA-BHC } \\
\text { BETA-BHC } \\
\text { DELTA-BHC } \\
\text { GAMMA-BHC (LINDANE) } \\
\text { MEPTACHLOR } \\
\text { ALORIN } \\
\text { HEPTACHLOR EPOXIDE } \\
\text { ENDOSULFAN I } \\
\text { DIELDRIM } \\
4,61-D D E \\
\text { ENDRII }\end{array}$ & $\begin{array}{l}U G / K G \\
U G / K G \\
U G / K G \\
U G / K G \\
U G / K G \\
U G / K G \\
U G / K G \\
U G / K G \\
U G / K G \\
U G / K G \\
U G / K G\end{array}$ & $\begin{array}{r}8.900 \\
8.900 \\
8.900 \\
8.900 \\
8.900 \\
8.900 \\
8.900 \\
8.900 \\
18.000 \\
18.000 \\
18.000\end{array}$ & $\begin{array}{l}u \\
u \\
u \\
u \\
u \\
u \\
u \\
u \\
u \\
u \\
u\end{array}$ \\
\hline
\end{tabular}


WHC-SD-EN-TI-279, Rev. 0

Lecation $307 \mathrm{RB}-1,399 \cdot 3-13$

\begin{tabular}{|c|c|c|c|}
\hline \multirow[t]{2}{*}{ Porcmeter } & \multirow{2}{*}{$\begin{array}{l}\begin{array}{l}\text { Sempl } \\
\text { Depth }\end{array} \\
\text { Units }\end{array}$} & \multicolumn{2}{|l|}{$\begin{array}{r}801656 \\
67.00\end{array}$} \\
\hline & & Resule & a \\
\hline 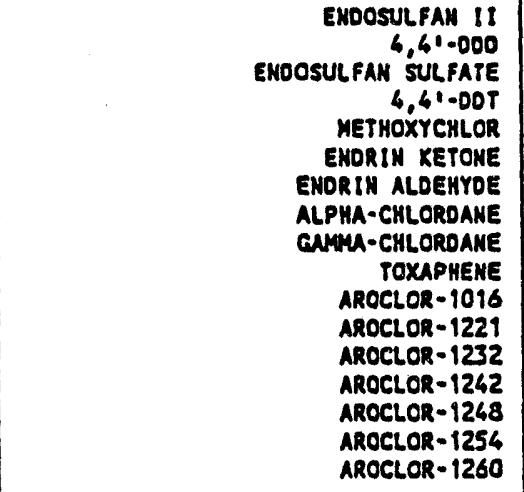 & $\begin{array}{l}\text { UG/KG } \\
\text { UG/KG } \\
\text { UG/KG } \\
\text { UG/KG } \\
\text { UG/KG } \\
\text { UG/KG } \\
\text { UG/KG } \\
\text { UG/KG } \\
\text { UG/KG } \\
\text { UG/KG } \\
\text { UG/KG } \\
\text { UG/KG } \\
\text { UG/KG } \\
\text { UG/KG } \\
\text { UG/KG } \\
\text { UG/KG }\end{array}$ & $\begin{array}{c}18.000 \\
18.000 \\
18.000 \\
18.000 \\
89.000 \\
18.000 \\
N / R \\
89.000 \\
89.000 \\
180.000 \\
89.000 \\
89.000 \\
89.000 \\
89.000 \\
89.000 \\
180.000 \\
180.000\end{array}$ & $\begin{array}{l}\mathbf{u} \\
\mathbf{u} \\
\mathbf{u} \\
\mathbf{u} \\
\mathbf{u} \\
\mathbf{u} \\
\mathbf{u} \\
\mathbf{u} \\
\mathbf{u} \\
\mathbf{u} \\
\mathbf{u} \\
\mathbf{u} \\
\mathbf{u} \\
\mathbf{u} \\
\mathbf{u}\end{array}$ \\
\hline
\end{tabular}


WHC-SD-EN-TI-279, Rev. 0

This page left intentionally blank. 
Location 307 RB-2, 399-3-14

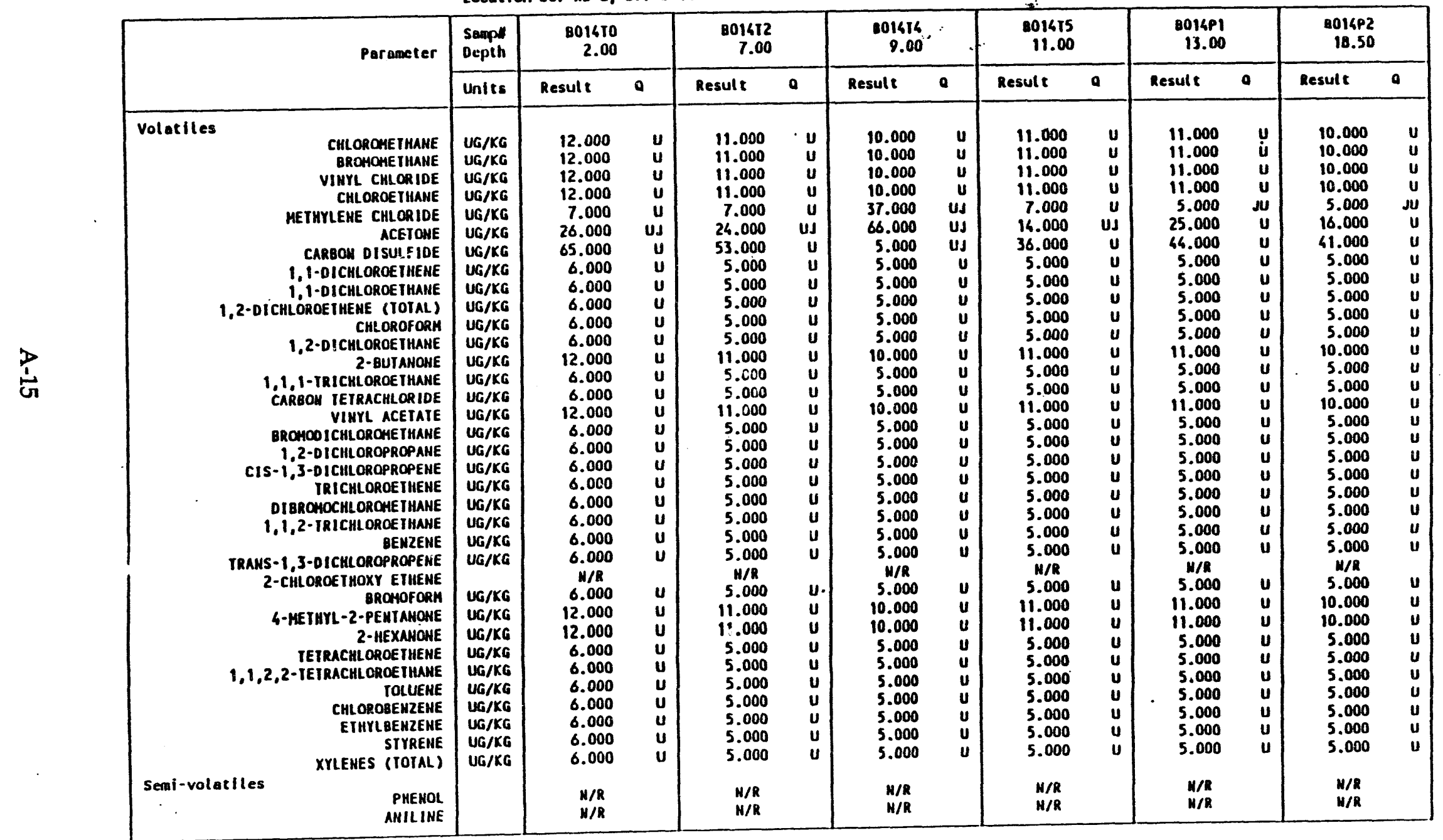


Location 307 R8-2, 399-3-16

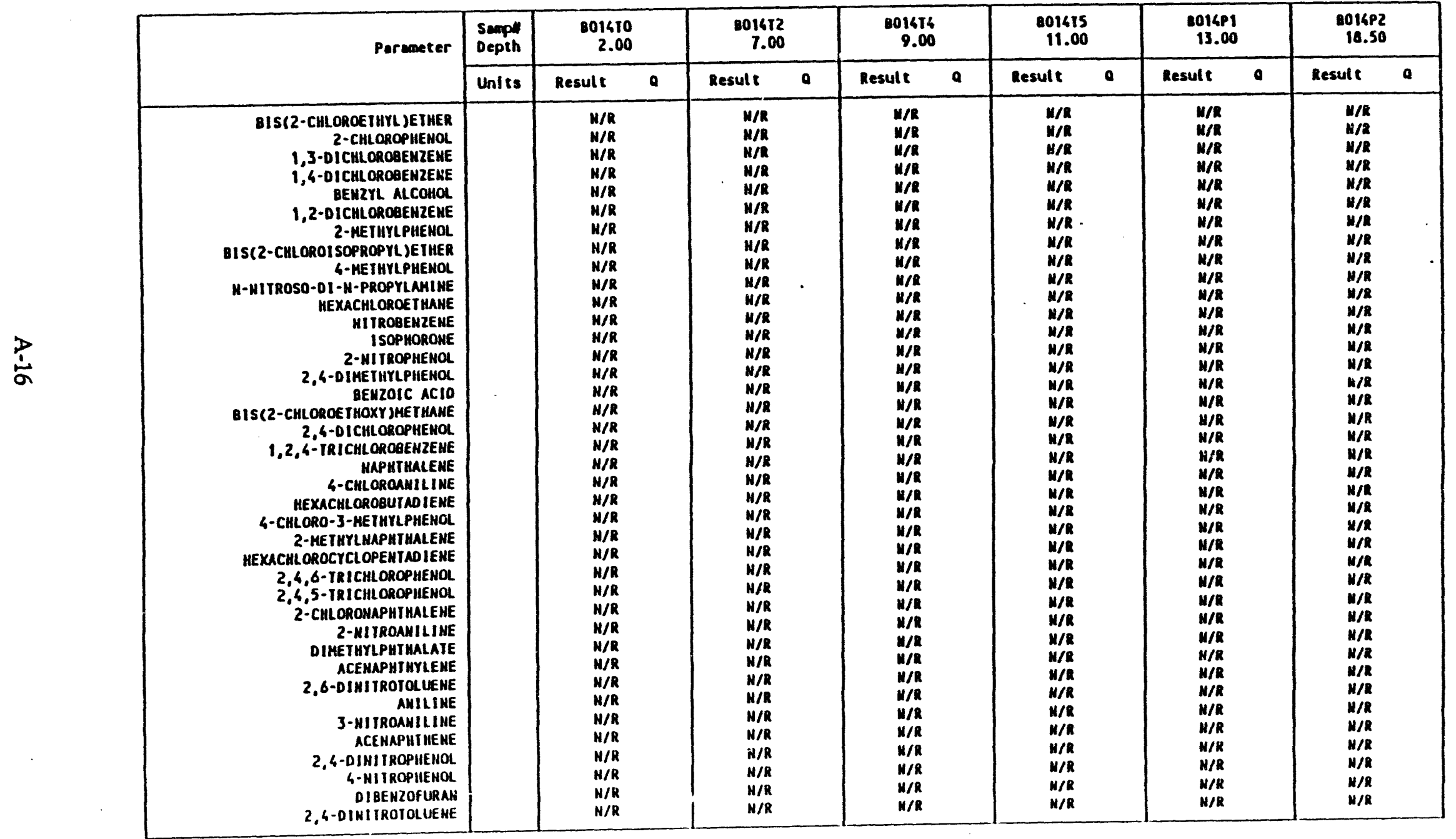


Location 307 RB-2, 399-3-16

\begin{tabular}{|c|c|c|c|c|c|c|c|c|c|c|c|c|c|}
\hline \multirow[t]{2}{*}{ Parameter } & \multirow{2}{*}{$\begin{array}{l}\text { Semp: } \\
\text { Depth }\end{array}$} & \multicolumn{2}{|l|}{$\begin{array}{r}201410 \\
2.00\end{array}$} & \multicolumn{2}{|l|}{$\begin{array}{r}801412 \\
7.00\end{array}$} & \multicolumn{2}{|l|}{$\begin{array}{r}001614 \\
8.00\end{array}$} & \multicolumn{2}{|l|}{$\begin{array}{r}001615 \\
11.00\end{array}$} & \multicolumn{2}{|l|}{$\begin{array}{r}1016 P 1 \\
13.00\end{array}$} & \multicolumn{2}{|l|}{$\begin{array}{r}0014 P 2 \\
10.50\end{array}$} \\
\hline & & Result & a & Result & a & Result & a & Result & a & Result & a & Result & a \\
\hline 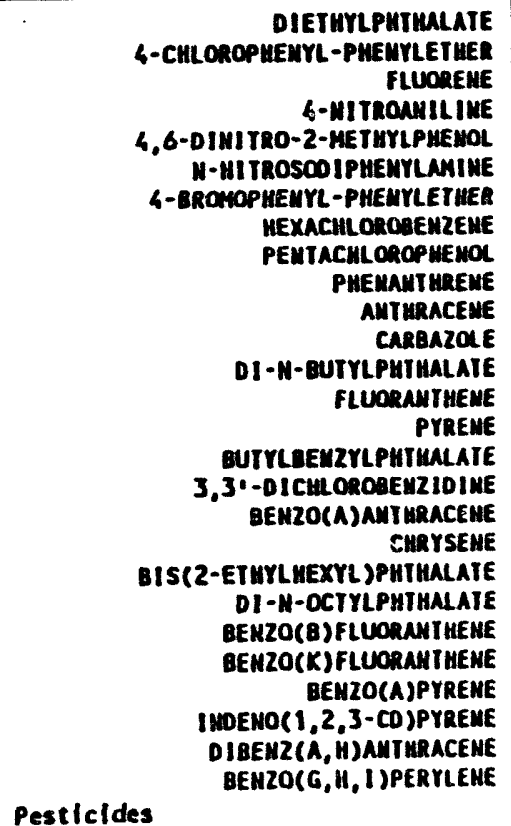 & & 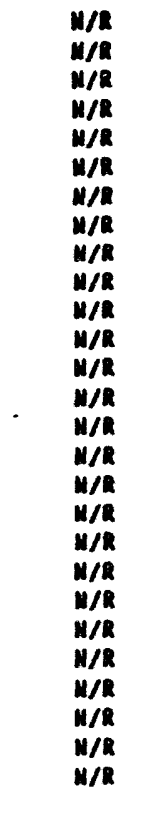 & & $\begin{array}{l}W / R \\
W / R \\
W / R \\
W / R \\
W / R \\
W / R \\
W / R \\
W / R \\
W / R \\
W / R \\
W / R \\
W / R \\
W / R \\
W / R \\
W / R \\
W / R \\
W / R \\
W / R \\
W / R \\
W / R \\
W / R \\
W / R \\
W / R \\
W / R \\
W / R \\
W / R \\
W / R\end{array}$ & & 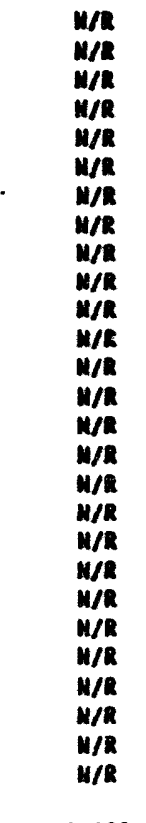 & & 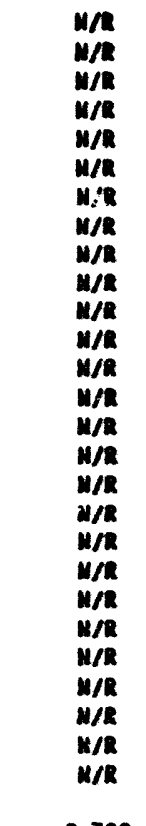 & & 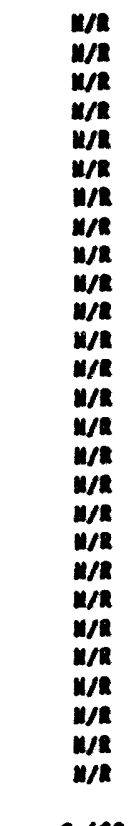 & & 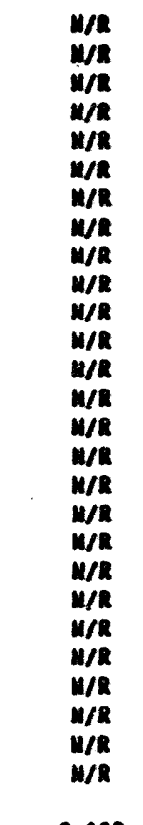 & \\
\hline 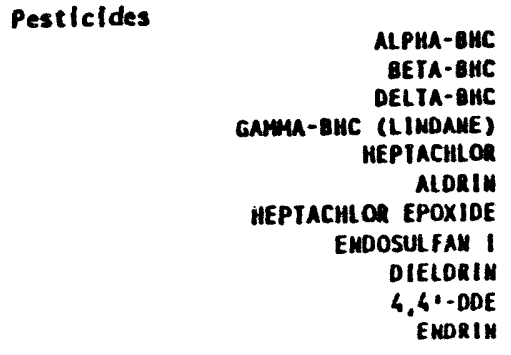 & $\begin{array}{l}\text { UG/KG } \\
\text { UG/KG } \\
\text { UG/KG } \\
\text { UG/KG } \\
\text { UG/KG } \\
\text { UG/KG } \\
\text { UG/KG } \\
\text { UG/KG } \\
\text { UG/KG } \\
\text { UG/KG } \\
\text { UG/KG }\end{array}$ & $\begin{array}{l}9.500 \\
9.500 \\
9.500 \\
9.500 \\
9.500 \\
9.500 \\
9.500 \\
9.500 \\
19.000 \\
19.000 \\
19.000\end{array}$ & $\begin{array}{l}u \\
u \\
u \\
u \\
u \\
u \\
u \\
u \\
u \\
u \\
u\end{array}$ & $\begin{array}{l}8.500 \\
8.500 \\
8.500 \\
8.500 \\
8.500 \\
8.500 \\
8.500 \\
8.500 \\
17.000 \\
17.000 \\
17.000\end{array}$ & $\begin{array}{l}u \\
u \\
u \\
u \\
u \\
u \\
u \\
u \\
u \\
u \\
u\end{array}$ & $\begin{array}{r}8.100 \\
8.100 \\
8.100 \\
8.100 \\
8.100 \\
8.100 \\
8.100 \\
8.100 \\
16.000 \\
16.000 \\
16.000\end{array}$ & $\begin{array}{l}\mathbf{u} \\
\mathbf{u} \\
\mathbf{u} \\
\mathbf{u} \\
\mathbf{u} \\
\mathbf{u} \\
\mathbf{u} \\
\mathbf{u} \\
\mathbf{u} \\
\mathbf{u} \\
\mathbf{u}\end{array}$ & $\begin{array}{r}8.300 \\
8.300 \\
8.300 \\
8.300 \\
8.300 \\
8.300 \\
8.300 \\
8.300 \\
17.000 \\
17.000 \\
17.000\end{array}$ & $\begin{array}{l}u \\
u \\
u \\
u \\
u \\
u \\
u \\
u \\
u \\
u \\
u\end{array}$ & $\begin{array}{l}8.400 \\
8.400 \\
8.400 \\
8.400 \\
8.400 \\
8.400 \\
8.400 \\
8.400 \\
17.000 \\
17.000 \\
17.000\end{array}$ & $\begin{array}{l}\mathbf{u} \\
\mathbf{u} \\
\mathbf{u} \\
\mathbf{u} \\
\mathbf{u} \\
\mathbf{u} \\
\mathbf{u} \\
\mathbf{u} \\
\mathbf{u} \\
\mathbf{u} \\
\mathbf{u}\end{array}$ & $\begin{array}{r}8.100 \\
8.100 \\
8.100 \\
8.100 \\
8.100 \\
8.100 \\
8.100 \\
8.100 \\
16.000 \\
16.000 \\
16.000\end{array}$ & $\begin{array}{l}y \\
y \\
y \\
y \\
y \\
y \\
y \\
y \\
y \\
u\end{array}$ \\
\hline
\end{tabular}


Location 307 R日-2, 399-3-16

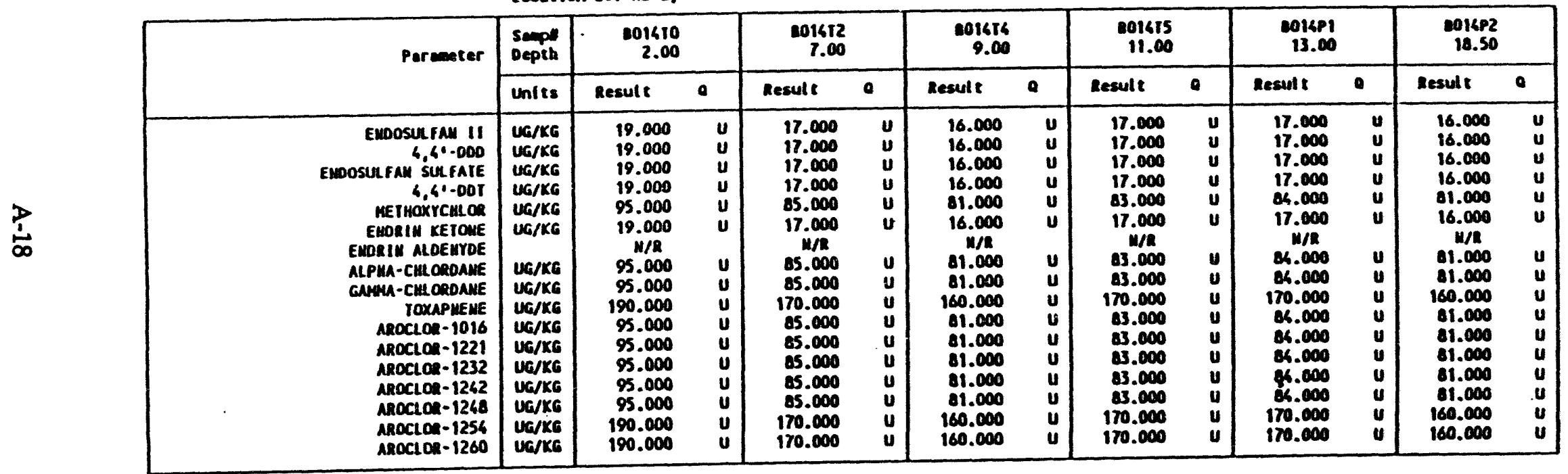




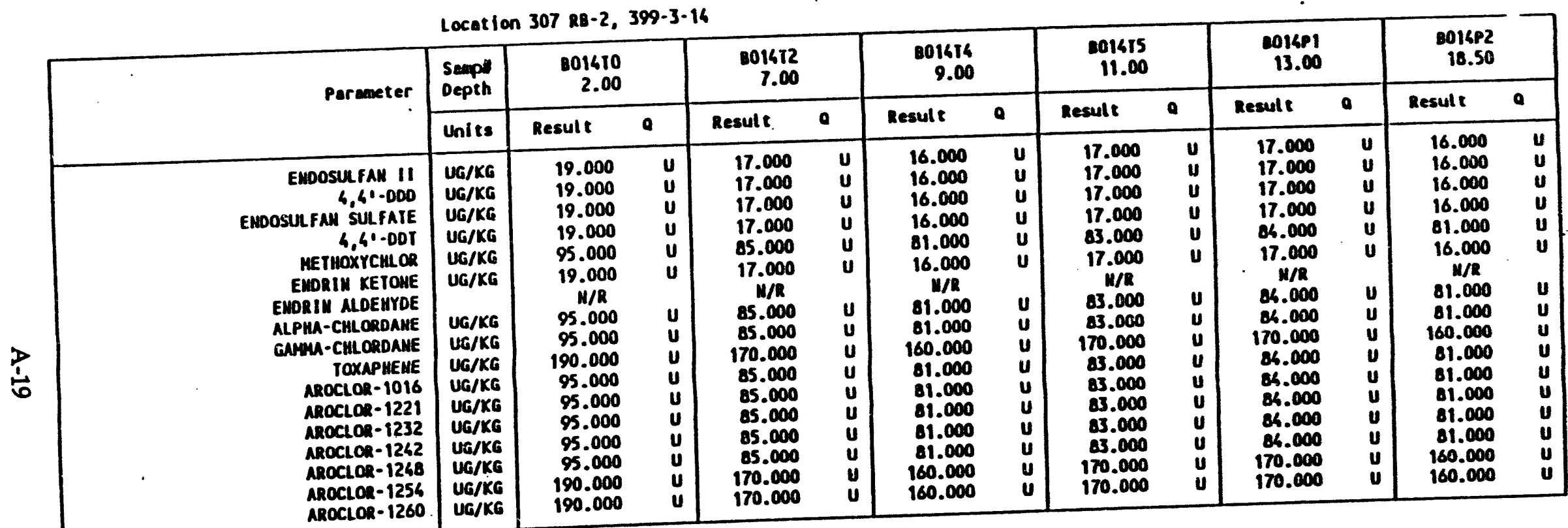


Location 307 RB-2, 399-3-14

\begin{tabular}{|c|c|c|c|c|c|c|c|c|c|c|c|c|c|}
\hline \multirow[t]{2}{*}{ Parameter } & \multirow{2}{*}{$\frac{\begin{array}{c}\text { Semp: } \\
\text { Depth }\end{array}}{\text { Unlis }}$} & \multicolumn{2}{|l|}{$\begin{array}{r}8014 P 3 \\
22.00\end{array}$} & \multicolumn{2}{|l|}{$\begin{array}{r}801 C B 3 \\
27.00\end{array}$} & \multicolumn{2}{|l|}{$\begin{array}{r}801 C B 4 \\
32.00\end{array}$} & \multicolumn{2}{|l|}{$\begin{array}{r}801 C B 7 \\
37.00\end{array}$} & \multicolumn{2}{|l|}{$\begin{array}{r}801 C 89 \\
42.00\end{array}$} & \multicolumn{2}{|l|}{$\begin{array}{r}801 C C 0 \\
67.00\end{array}$} \\
\hline & & Result & & Result & $a$ & Result & 0 & Result & a & Result & $a$ & Result & $a$ \\
\hline 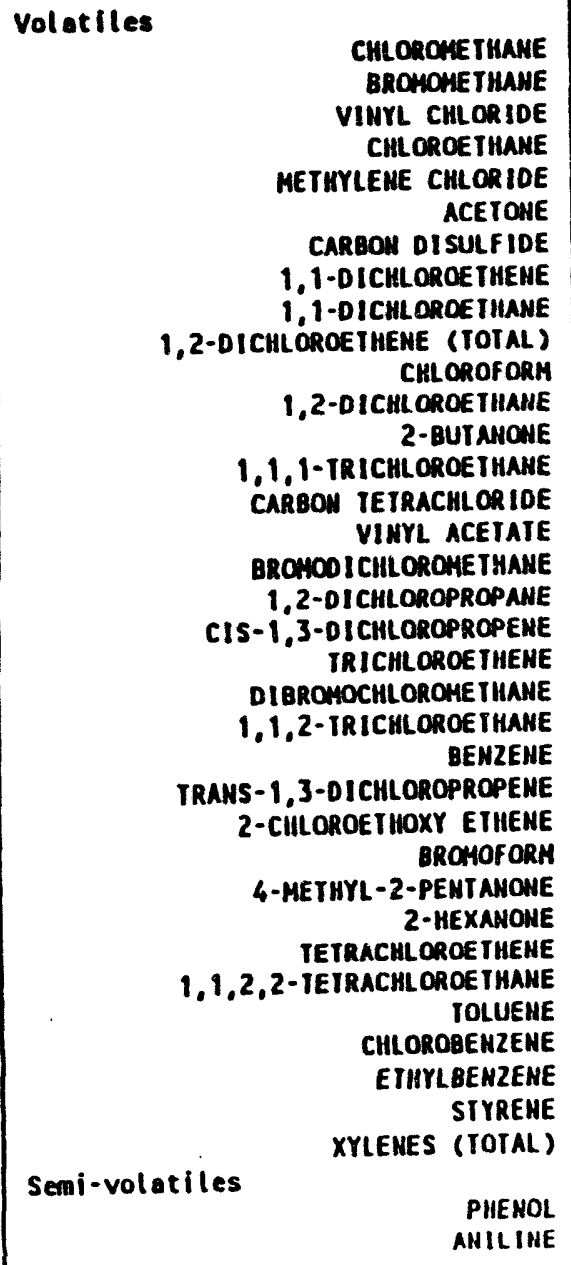 & $\begin{array}{l}U G / K G \\
U G / K G \\
U G / K G \\
U G / K G \\
U G / K G \\
U G / K G \\
U G / K G \\
U G / K G \\
U G / K G \\
U G / K G \\
U G / K G \\
U G / K G \\
U G / K G \\
U G / K G \\
U G / K G \\
U G / K G \\
U G / K G \\
U G / K G \\
U G / K G \\
U G / K G \\
U G / K G \\
U G / K G \\
U G / K G \\
U G / K G \\
\\
U G / K G \\
U G / K G \\
U G / K G \\
U G / K G \\
U G / K G \\
U G / K G \\
U G / K G \\
U G / K G \\
U G / K G \\
U G / K G\end{array}$ & $\begin{array}{r}11.000 \\
11.000 \\
11.000 \\
11.000 \\
6.000 \\
24.000 \\
4.000 \\
5.000 \\
5.000 \\
5.000 \\
5.000 \\
5.000 \\
11.000 \\
5.000 \\
5.000 \\
11.000 \\
5.000 \\
5.000 \\
5.000 \\
5.000 \\
5.000 \\
5.000 \\
5.000 \\
5.000 \\
11 / R \\
5.000 \\
11.000 \\
11.000 \\
5.000 \\
5.000 \\
5.000 \\
5.000 \\
5.000 \\
5.000 \\
5.000 \\
\\
N / R \\
H / R\end{array}$ & $\begin{array}{l}u \\
u \\
u \\
u \\
u \\
u \\
u \\
u \\
u \\
u \\
u \\
u \\
u \\
u \\
u \\
u \\
u \\
u \\
u \\
u \\
u \\
u \\
u \\
u \\
u \\
u \\
u \\
u \\
u \\
u \\
u \\
u \\
u \\
u\end{array}$ & $\begin{array}{c}12.000 \\
12.000 \\
12.000 \\
12.000 \\
4.000 \\
6.000 \\
1.000 \\
6.000 \\
6.000 \\
6.000 \\
6.000 \\
6.000 \\
12.000 \\
6.000 \\
6.000 \\
12.000 \\
6.000 \\
6.000 \\
6.000 \\
6.000 \\
6.000 \\
6.000 \\
6.000 \\
6.000 \\
H / R \\
6.000 \\
12.000 \\
12.000 \\
6.000 \\
6.000 \\
6.000 \\
6.000 \\
6.000 \\
6.000 \\
6.000 \\
\\
H / R \\
H / R\end{array}$ & $\begin{array}{c}\mathbf{u} \\
\mathbf{u} \\
\mathbf{u} \\
\mathbf{u} \\
\mathbf{u} \\
\mathbf{u} \\
\mathbf{u} \\
\mathbf{u} \\
\mathbf{u} \\
\mathbf{u} \\
\mathbf{u} \\
\mathbf{u} \\
\mathbf{u} \\
\mathbf{u} \\
\mathbf{u} \\
\mathbf{u} \\
\mathbf{u} \\
\mathbf{u} \\
\mathbf{u} \\
\mathbf{u} \\
\mathbf{u} \\
\mathbf{u} \\
\mathbf{u} \\
\mathbf{u} \\
\mathbf{u} \\
\mathbf{u} \\
\mathbf{u} \\
\mathbf{u} \\
\mathbf{u} \\
\mathbf{u} \\
\mathbf{u} \\
\mathbf{u} \\
\mathbf{u}\end{array}$ & $\begin{array}{c}10.000 \\
10.000 \\
10.000 \\
10.000 \\
3.000 \\
5.000 \\
5.000 \\
5.000 \\
5.000 \\
5.000 \\
5.000 \\
5.000 \\
10.000 \\
5.000 \\
5.000 \\
10.000 \\
5.000 \\
5.000 \\
5.000 \\
5.000 \\
5.000 \\
5.000 \\
5.000 \\
5.000 \\
N / R \\
5.000 \\
10.000 \\
10.000 \\
5.000 \\
5.000 \\
5.000 \\
5.000 \\
5.000 \\
5.000 \\
5.000 \\
\\
H / R \\
11 / R\end{array}$ & $\begin{array}{c}\mathbf{u} \\
\mathbf{u} \\
\mathbf{u} \\
\mathbf{u} \\
\mathbf{u} \\
\mathbf{u} \\
\mathbf{u} \\
\mathbf{u} \\
\mathbf{u} \\
\mathbf{u} \\
\mathbf{u} \\
\mathbf{u} \\
\mathbf{u} \\
\mathbf{u} \\
\mathbf{u} \\
\mathbf{u} \\
\mathbf{u} \\
\mathbf{u} \\
\mathbf{u} \\
\mathbf{u} \\
\mathbf{u} \\
\mathbf{u} \\
\mathbf{u} \\
\mathbf{u} \\
\mathbf{u} \\
\mathbf{u} \\
\mathbf{u} \\
\mathbf{u} \\
\mathbf{u} \\
\mathbf{u} \\
\mathbf{u} \\
\mathbf{u} \\
\mathbf{u}\end{array}$ & $\begin{array}{c}11.000 \\
11.000 \\
11.000 \\
11.000 \\
15.000 \\
26.000 \\
5.000 \\
5.000 \\
5.000 \\
5.000 \\
5.000 \\
5.000 \\
11.000 \\
5.000 \\
5.000 \\
11.000 \\
5.000 \\
5.000 \\
5.000 \\
5.000 \\
5.000 \\
5.000 \\
5.000 \\
5.000 \\
H / R \\
5.000 \\
11.000 \\
11.000 \\
5.000 \\
5.000 \\
5.000 \\
5.000 \\
5.000 \\
5.000 \\
5.000 \\
\\
11 / R \\
H / R\end{array}$ & $\begin{array}{l}\text { UJ } \\
\text { UJ } \\
\text { UJ } \\
\text { UJ } \\
\text { UJ } \\
\text { UJ } \\
\text { UJ } \\
\text { UJ } \\
\text { UJ } \\
\text { UJ } \\
\text { UJ } \\
\text { UJ } \\
\text { UJ } \\
\text { UJ } \\
\text { UJ } \\
\text { UJ } \\
\text { UJ } \\
\text { UJ } \\
\text { UJ } \\
\text { UJ } \\
\text { UJ } \\
\text { UJ } \\
\text { UJ } \\
\text { UJ } \\
\text { UJ } \\
\text { UJ } \\
\text { UJ } \\
\text { UJ } \\
\text { UJ } \\
\text { UJ } \\
\text { UJ } \\
\text { UJ } \\
\text { UJ } \\
\text { UJ }\end{array}$ & $\begin{array}{r}11.000 \\
11.000 \\
11.000 \\
11.000 \\
18.000 \\
40.000 \\
5.000 \\
5.000 \\
5.000 \\
5.000 \\
5.000 \\
5.000 \\
11.000 \\
5.000 \\
5.000 \\
11.000 \\
5.000 \\
5.000 \\
5.000 \\
5.000 \\
5.000 \\
5.000 \\
5.000 \\
5.000 \\
11 / 8 \\
5.000 \\
11.000 \\
11.000 \\
5.000 \\
5.000 \\
5.000 \\
5.000 \\
5.000 \\
5.000 \\
5.000 \\
\\
11 / R \\
11 / 8\end{array}$ & 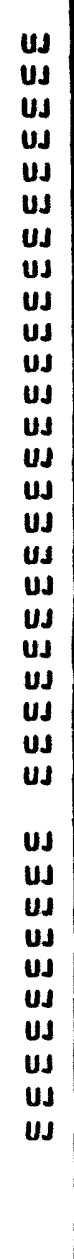 & $\begin{array}{c}11.000 \\
11.000 \\
11.000 \\
11.000 \\
61.000 \\
67.000 \\
5.000 \\
5.000 \\
5.000 \\
5.000 \\
5.000 \\
5.000 \\
11.000 \\
5.000 \\
5.000 \\
11.000 \\
5.000 \\
5.000 \\
5.000 \\
5.000 \\
5.000 \\
5.000 \\
5.000 \\
5.000 \\
11 / R \\
5.000 \\
11.000 \\
11.000 \\
5.000 \\
5.000 \\
5.000 \\
5.000 \\
5.000 \\
5.000 \\
5.000 \\
\\
H / R \\
H / R\end{array}$ & 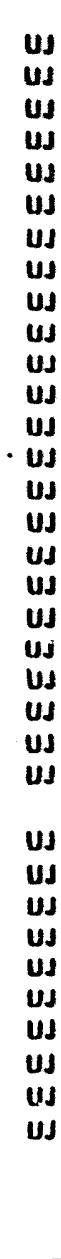 \\
\hline
\end{tabular}


Location 307 RB-2, 399-3-14

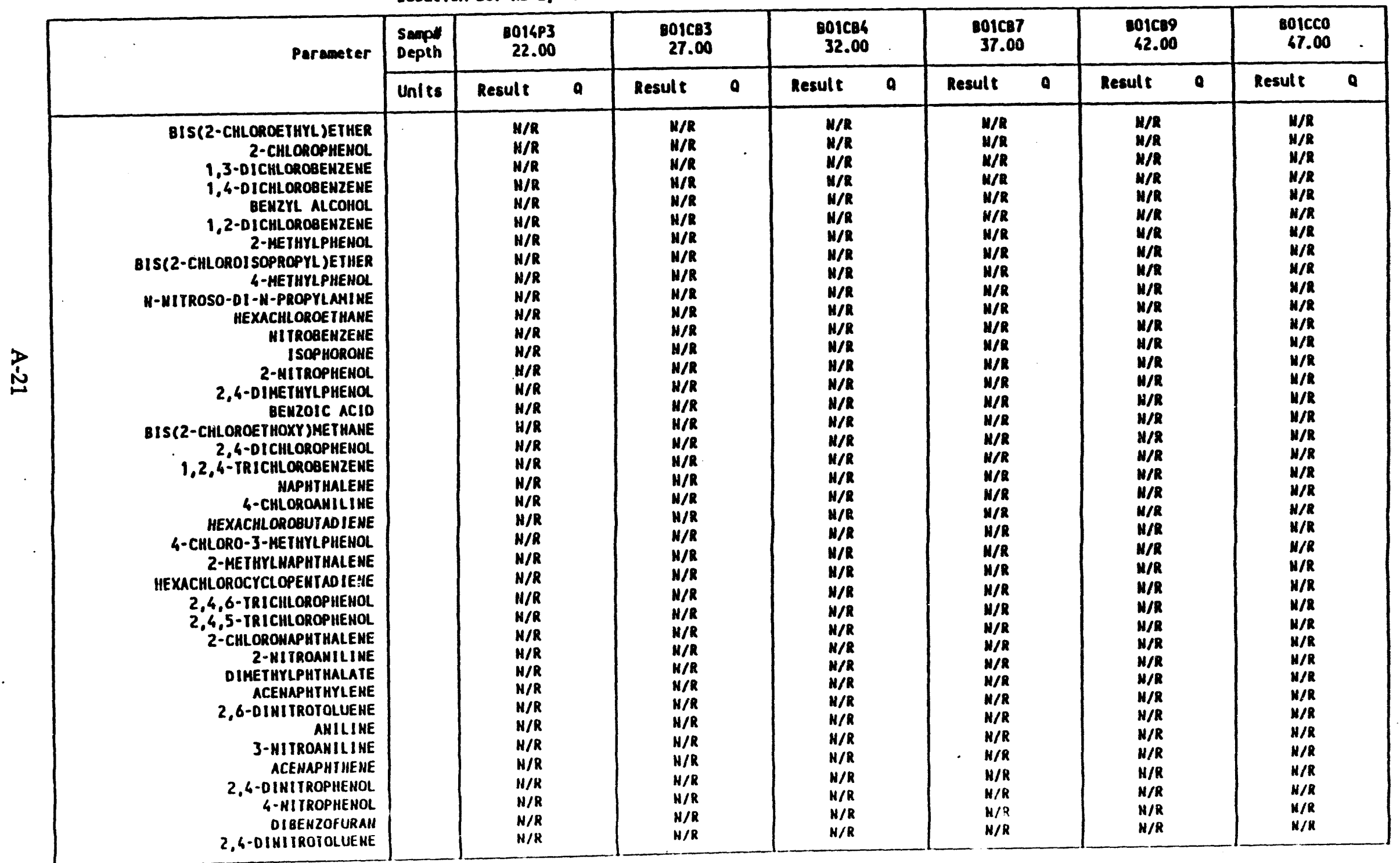


Location 307 RB-2, 399-3-14

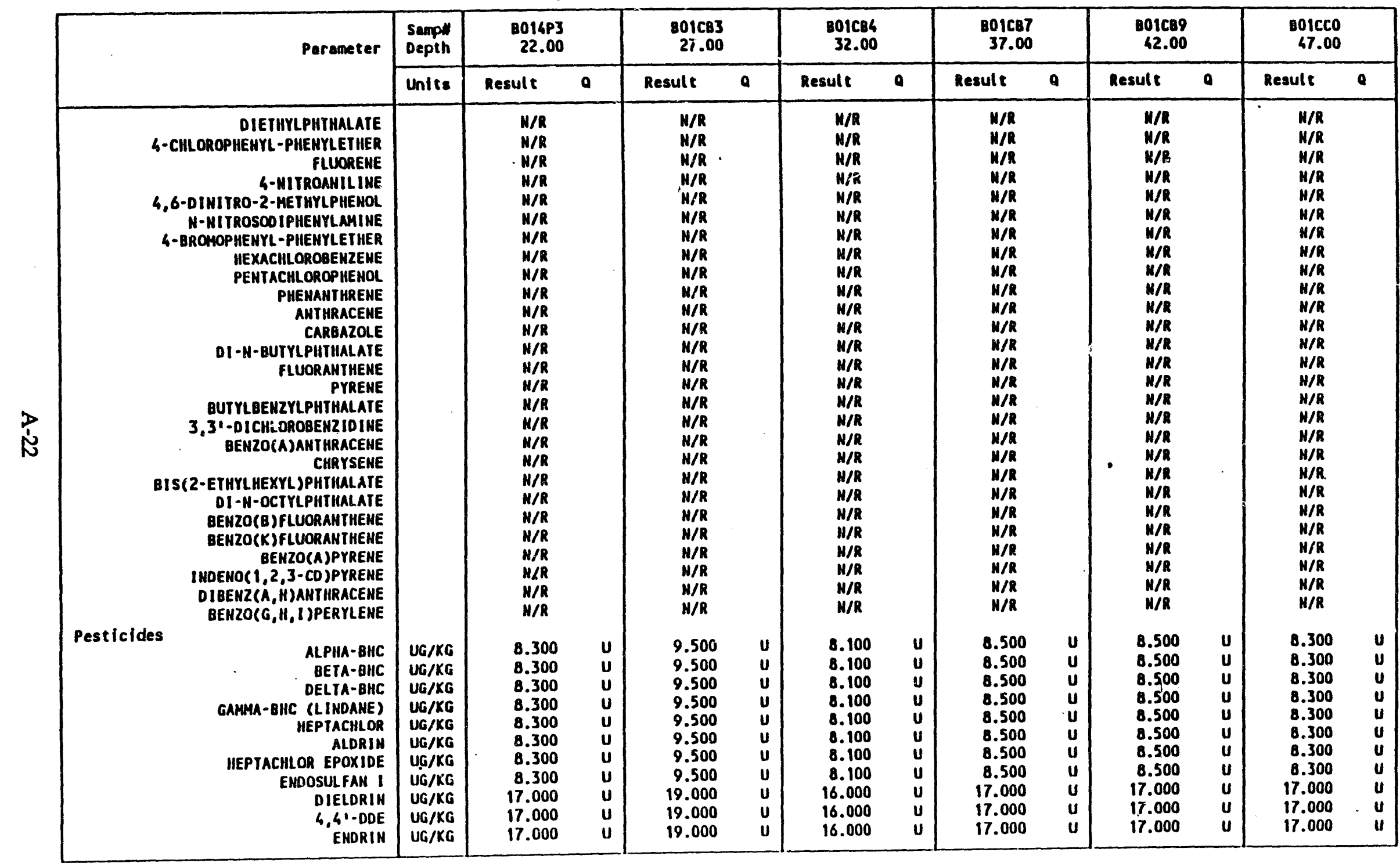




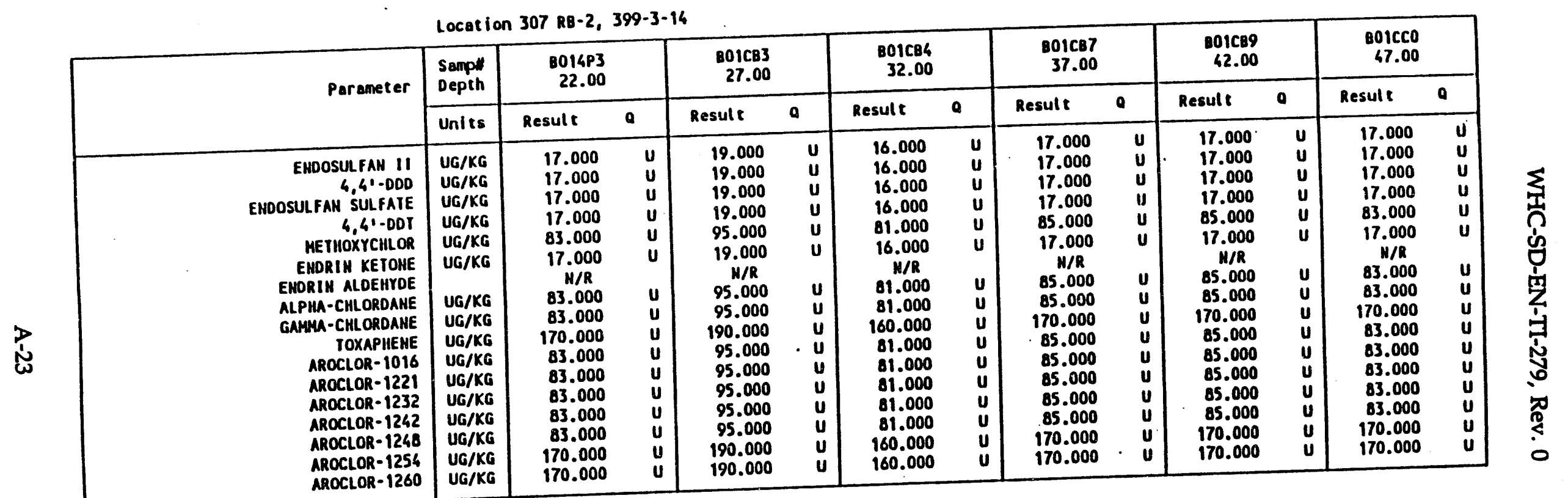


WHC-SD-EN-TI-279, Rev. 0

Location 307 RB-2, 399-3-14

\begin{tabular}{|c|c|c|c|c|c|}
\hline \multirow[t]{2}{*}{ Parameter } & \multirow{2}{*}{$\begin{array}{c}\text { Samp: } \\
\text { Depth }\end{array}$} & \multicolumn{2}{|l|}{$\begin{array}{r}8016 c 1 \\
49.00\end{array}$} & \multicolumn{2}{|l|}{$\begin{array}{r}B 016 C 2 \\
56.00\end{array}$} \\
\hline & & Result & 0 & Result & 0 \\
\hline 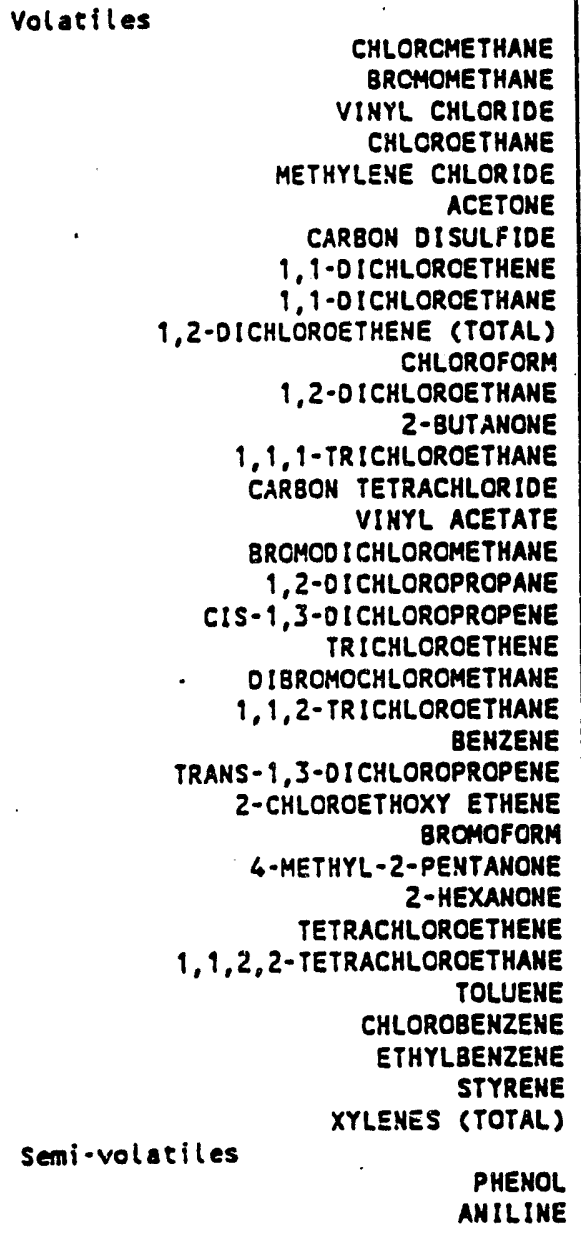 & $\begin{array}{l}U G / K G \\
U G / K G \\
U G / K G \\
U G / K G \\
U G / K G \\
U G / K G \\
U G / K G \\
U G / K G \\
U G / K G \\
U G / K G \\
U G / K G \\
U G / K G \\
U G / K G \\
U G / K G \\
U G / K G \\
U G / K G \\
U G / K G \\
U G / K G \\
U G / K G \\
U G / K G \\
U G / K G \\
U G / K G \\
U G / K G \\
U G / K G \\
U G / K G \\
U G / K G \\
U G / R G \\
U G / K G \\
U G / K G \\
U G / K G \\
U G / K G \\
U G / K G \\
U G / K G \\
U G / K G\end{array}$ & $\begin{array}{r}11.000 \\
11.000 \\
11.000 \\
11.000 \\
65.000 \\
82.000 \\
6.000 \\
6.000 \\
6.000 \\
6.000 \\
6.000 \\
6.000 \\
11.000 \\
6.000 \\
6.000 \\
11.000 \\
6.000 \\
6.000 \\
6.000 \\
6.000 \\
6.000 \\
6.00 \\
6.000 \\
6.000 \\
6.000 \\
H / R \\
\quad H \quad \\
6.000 \\
11.000 \\
11.000 \\
6.000 \\
6.000 \\
6\end{array}$ & 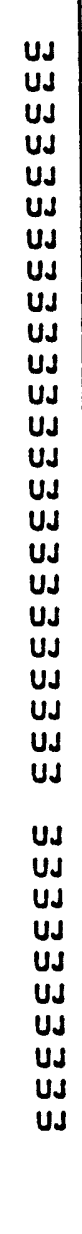 & $\begin{array}{c}11.000 \\
11.000 \\
11.000 \\
11.000 \\
32.000 \\
66.000 \\
6.000 \\
6.000 \\
6.000 \\
6.000 \\
6.000 \\
6.000 \\
11.000 \\
6.000 \\
6.000 \\
11.000 \\
6.000 \\
6.000 \\
6.000 \\
6.000 \\
6.000 \\
6.000 \\
6.000 \\
6.000 \\
6.000 \\
\quad 1 / R \\
\quad 1 / R \\
6.000 \\
11.000 \\
11.000 \\
6.000 \\
6.000 \\
6.000 \\
6.000 \\
6\end{array}$ & 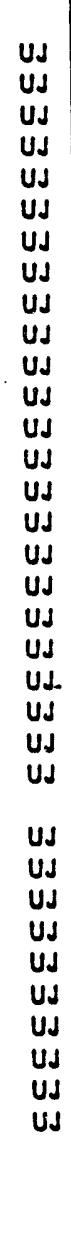 \\
\hline
\end{tabular}


Location 307 RB-2, 399-3-16

\begin{tabular}{|c|c|c|c|}
\hline \multirow[t]{2}{*}{ Parameter } & \multirow{2}{*}{$\begin{array}{l}\text { Sampit } \\
\text { Depth }\end{array}$} & $\begin{array}{r}8016 C 1 \\
49.00\end{array}$ & $\begin{array}{r}8016 C 2 \\
56.00\end{array}$ \\
\hline & & Result & Resule \\
\hline 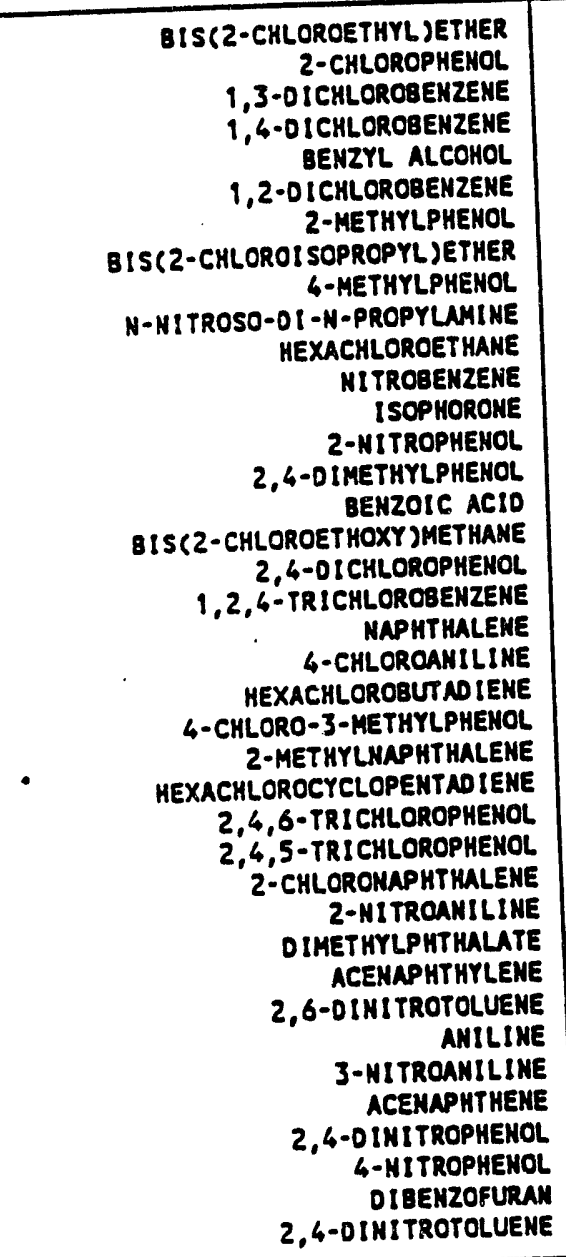 & & 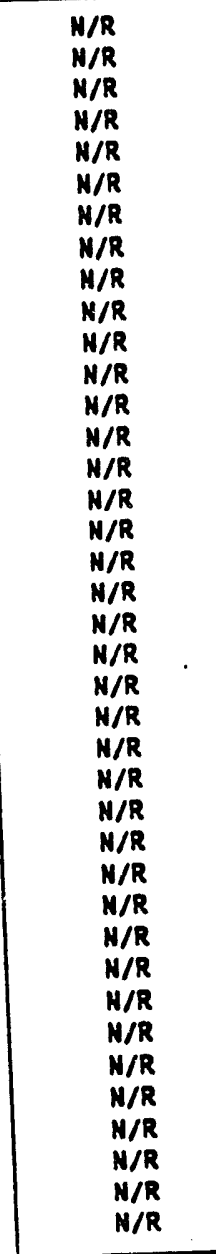 & $\begin{array}{l}H / R \\
H / R \\
H / R \\
H / R \\
H / R \\
H / R \\
H / R \\
H / R \\
H / R \\
H / R \\
H / R \\
H / R \\
H / R \\
H / R \\
H / R \\
H / R \\
H / R \\
H / R \\
H / R \\
H / R \\
H / R \\
H / R \\
H / R \\
H / R \\
H / R \\
H / R \\
H / R \\
H / R \\
H / R \\
H / R \\
H / R \\
H / R \\
H / R \\
H / R \\
H / R \\
H / R \\
H / R \\
H / R \\
H / R\end{array}$ \\
\hline
\end{tabular}


WHC-SD-EN-TI-279, Rev. 0

Locstion 307 RB-2, 399-3-14

\begin{tabular}{|c|c|c|c|c|c|}
\hline \multirow[t]{2}{*}{ Parameter } & \multirow{2}{*}{$\begin{array}{l}\text { Semp: } \\
\text { Depth }\end{array}$} & \multicolumn{2}{|l|}{$\begin{array}{r}8016 c 1 \\
49.00\end{array}$} & \multicolumn{2}{|l|}{$\begin{array}{r}8010 C 2 \\
56.00\end{array}$} \\
\hline & & Resule & 0 & Result & a \\
\hline 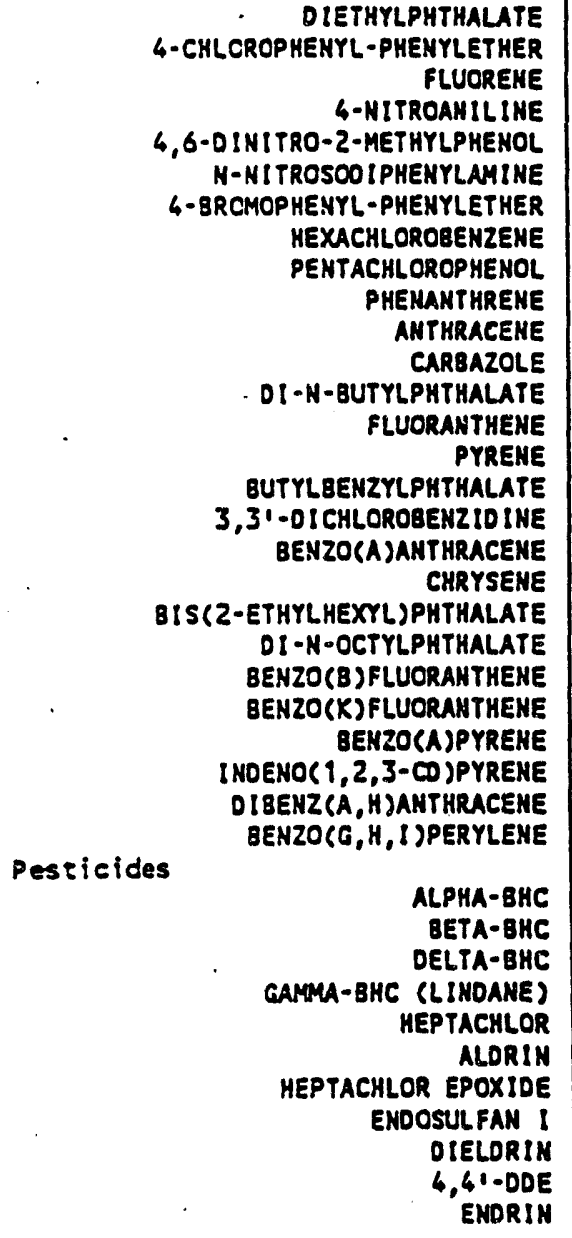 & $\begin{array}{l} \\
\text { UG/KG } \\
\text { UG/KG } \\
\text { UG/KG } \\
\text { UG/KG } \\
\text { UG/KG } \\
\text { UG/KG } \\
\text { UG/KG } \\
\text { UG/KG } \\
\text { UG/KG } \\
\text { UG/KG } \\
\text { UG/KG }\end{array}$ & $\begin{array}{l}H / R \\
H / R \\
N / R \\
N / R \\
H / R \\
N / R \\
N / R \\
N / R \\
N / R \\
N / R \\
N / R \\
N / R \\
N / R \\
N / R \\
H / R \\
H / R \\
N / R \\
N / R \\
N / R \\
H / R \\
H / R \\
N / R \\
N / R \\
N / R \\
N / R \\
N / R \\
H / R \\
9.000 \\
9.000 \\
9.000 \\
9.000 \\
9.000 \\
9.000 \\
9.000 \\
9.000 \\
18.000 \\
18.000 \\
18.000\end{array}$ & $\begin{array}{l}U \\
U \\
U \\
U \\
U \\
U \\
U \\
U \\
U \\
U \\
U\end{array}$ & $\begin{array}{c}N / R \\
N / R \\
N / R \\
N / R \\
N / R \\
N / R \\
N / R \\
H / R \\
N / R \\
N / R \\
N / R \\
N / R \\
N / R \\
N / R \\
N / R \\
N / R \\
N / R \\
N / R \\
N / R \\
N / R \\
N / R \\
N / R \\
N / R \\
N / R \\
N / R \\
N / R \\
N / R \\
N / R \\
8.600 \\
8.600 \\
8.600 \\
8.600 \\
8.600 \\
8.600 \\
8.600 \\
8.600 \\
17.000 \\
17.000 \\
17.000\end{array}$ & $\begin{array}{l}U \\
U \\
U \\
U \\
U \\
U \\
U \\
U \\
U \\
U \\
U\end{array}$ \\
\hline
\end{tabular}


WHC-SD-EN-TI-279, Rev. 0

Location 307 R8-2, 399-3-16

\begin{tabular}{|c|c|c|c|c|c|}
\hline \multirow[t]{2}{*}{ Paraneter } & \multirow{2}{*}{$\begin{array}{l}\text { Samp" } \\
\text { Depth }\end{array}$} & \multicolumn{2}{|l|}{$\begin{array}{r}\text { Bo1ccl } \\
49.00\end{array}$} & \multicolumn{2}{|l|}{$\begin{array}{r}801 C 62 \\
56.00\end{array}$} \\
\hline & & Result & $a$ & Result & 0 \\
\hline 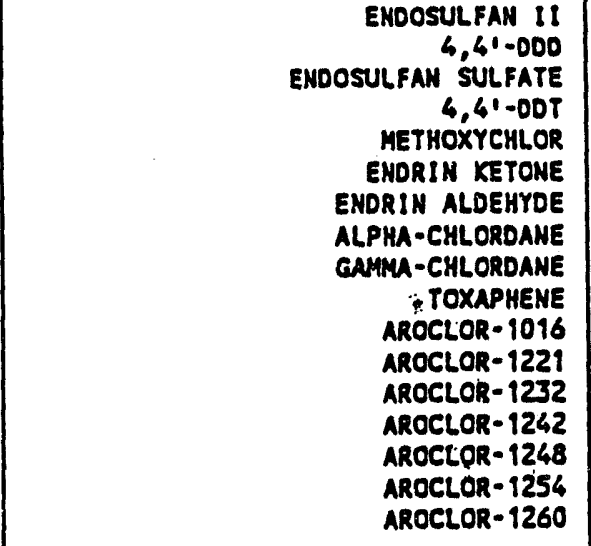 & $\begin{array}{l}\text { UG/KG } \\
\text { UG/KG } \\
U G / K G \\
\text { UG/KG } \\
U G / K G \\
\text { UG/KG } \\
\text { UG/KG } \\
U G / K G \\
U G / K G \\
\text { UG/KG } \\
\text { UG/KG } \\
\text { UG/KG } \\
\text { UG/KG } \\
\text { UG/KG } \\
\text { UG/KG } \\
\text { UG/KG }\end{array}$ & $\begin{array}{c}18.000 \\
18.000 \\
18.000 \\
18.000 \\
90.000 \\
18.000 \\
11 / R \\
90.000 \\
90.000 \\
180.000 \\
90.000 \\
90.000 \\
90.000 \\
90.000 \\
90.000 \\
180.000 \\
180.000\end{array}$ & $\begin{array}{l}u \\
u \\
u \\
u \\
u \\
u \\
u \\
u \\
u \\
u \\
u \\
u \\
u \\
u \\
u \\
u\end{array}$ & $\begin{array}{c}17.000 \\
17.000 \\
17.000 \\
17.000 \\
86.000 \\
17.000 \\
1 / R \\
86.000 \\
86.000 \\
170.000 \\
86.000 \\
86.000 \\
86.000 \\
86.000 \\
86.000 \\
170.000 \\
170.000\end{array}$ & $\begin{array}{l}u \\
u \\
u \\
u \\
u \\
u \\
u \\
u \\
u \\
u \\
u \\
u \\
u \\
u \\
u \\
U\end{array}$ \\
\hline
\end{tabular}


$8 Z-\forall$

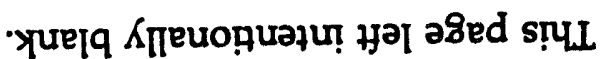

0 :АәУ '6LZ-IL-NG-CS-DHM 
Location $307 \mathrm{~T}-1,399-3-15$

\begin{tabular}{|c|c|c|c|c|c|c|c|c|c|c|c|c|c|}
\hline \multirow[t]{2}{*}{ Paraneter } & \multirow{2}{*}{$\begin{array}{l}\text { Sampk } \\
\text { Depth }\end{array}$} & \multicolumn{2}{|l|}{$\begin{array}{r}801 \text { CF9 } \\
4.60\end{array}$} & \multicolumn{2}{|l|}{$\begin{array}{r}801660 \\
7.00\end{array}$} & \multicolumn{2}{|l|}{$\begin{array}{r}801 C G 2 \\
9.00\end{array}$} & \multicolumn{2}{|l|}{$\begin{array}{r}\text { Bo1cG4 } \\
11.40\end{array}$} & \multicolumn{2}{|l|}{$\begin{array}{r}801 c 05 \\
13.60\end{array}$} & \multicolumn{2}{|l|}{$\begin{array}{r}801 c 66 \\
15.00\end{array}$} \\
\hline & & Result & Q & Result & a & Result & a & Result & a & Result & a & Result & $a$ \\
\hline 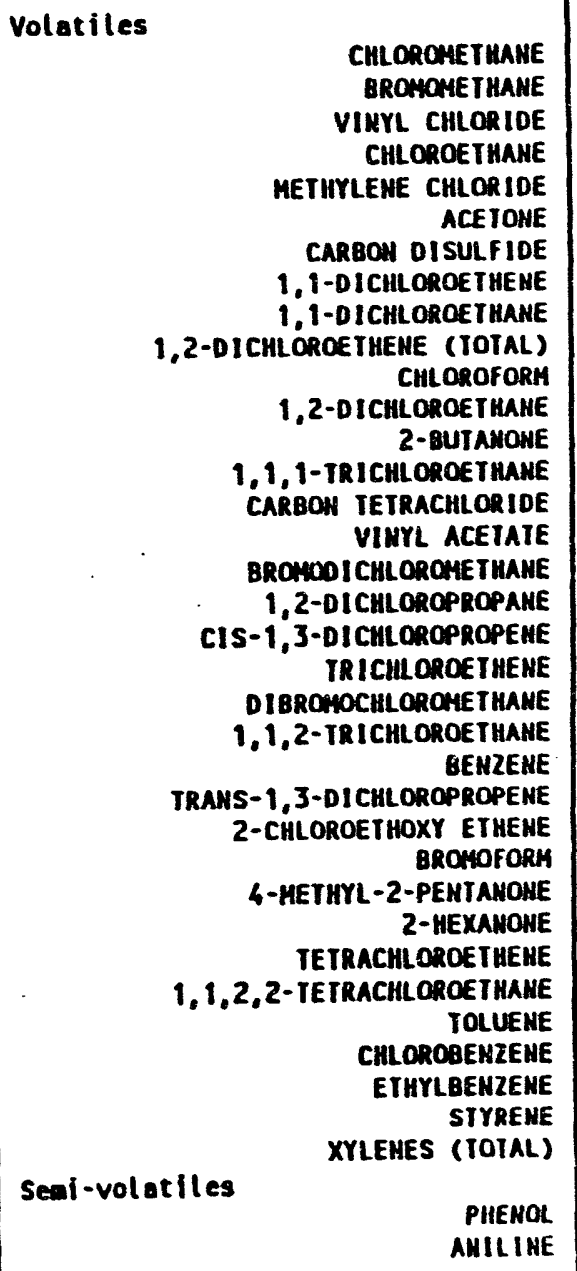 & $\begin{array}{l}\text { UG/KG } \\
U G / K G \\
U G / K G \\
U G / K G \\
U G / K G \\
U G / K G \\
U G / K G \\
U G / K G \\
U G / K G \\
U G / K G \\
U G / K G \\
U G / K G \\
U G / K G \\
U G / K G \\
U G / K G \\
U G / K G \\
U G / K G \\
U G / K G \\
U G / K G \\
U G / K G \\
U G / K G \\
U G / K G \\
U G / K G \\
U G / K G \\
U G / K G \\
U G / K G \\
U G / K G \\
U G / K G \\
U G / K G \\
U G / K G \\
U G / K G \\
U G / K G \\
U G / K G \\
U G / K G\end{array}$ & $\begin{array}{r}11.000 \\
11.000 \\
11.000 \\
11.000 \\
29.000 \\
21.000 \\
6.000 \\
5.000 \\
5.000 \\
5.000 \\
5.000 \\
5.000 \\
11.000 \\
5.000 \\
5.000 \\
11.000 \\
5.000 \\
5.000 \\
5.000 \\
5.000 \\
5.000 \\
5.000 \\
5.000 \\
5.000 \\
H / R \\
5.000 \\
11.000 \\
11.000 \\
5.000 \\
5.000 \\
5.000 \\
5.000 \\
5.000 \\
5.000 \\
5.000 \\
\\
H / R \\
W / R\end{array}$ & $\begin{array}{c}\mathbf{U} \\
\mathbf{u} \\
\mathbf{u} \\
\mathbf{u} \\
\mathbf{u} \\
\mathbf{u} \mathbf{u} \\
\mathbf{u} \\
\mathbf{u} \\
\mathbf{u} \\
\mathbf{u} \\
\mathbf{u} \\
\mathbf{u} \\
\mathbf{u} \\
\mathbf{u} \\
\mathbf{u} \\
\mathbf{u} \\
\mathbf{u} \\
\mathbf{u} \\
\mathbf{u} \\
\mathbf{u} \\
\mathbf{u} \\
\mathbf{u} \\
\mathbf{u} \\
\mathbf{u} \\
\mathbf{u} \\
\mathbf{u} \\
\mathbf{u} \\
\mathbf{u} \\
\mathbf{u} \\
\mathbf{u} \\
\mathbf{u} \\
\mathbf{u} \\
\mathbf{u} \\
\mathbf{u}\end{array}$ & $\begin{array}{c}10.000 \\
10.000 \\
10.000 \\
10.000 \\
29.000 \\
19.000 \\
5.000 \\
5.000 \\
5.000 \\
5.000 \\
5.000 \\
5.000 \\
10.000 \\
5.000 \\
5.000 \\
10.000 \\
5.000 \\
5.000 \\
5.000 \\
5.000 \\
5.000 \\
5.000 \\
5.000 \\
5.000 \\
N / R \\
5.000 \\
10.000 \\
10.000 \\
5.000 \\
5.000 \\
5.000 \\
5.000 \\
5.000 \\
5.000 \\
5.000 \\
\\
H / R \\
H / R\end{array}$ & $\begin{array}{c}\mathbf{u} \\
\mathbf{u} \\
\mathbf{u} \\
\mathbf{u} \\
\mathbf{u} \\
\mathbf{u} \\
\mathbf{u} \\
\mathbf{u} \\
\mathbf{u} \\
\mathbf{u} \\
\mathbf{u} \\
\mathbf{u} \\
\mathbf{u} \\
\mathbf{u} \\
\mathbf{u} \\
\mathbf{u} \\
\mathbf{u} \\
\mathbf{u} \\
\mathbf{u} \\
\mathbf{u} \\
\mathbf{u} \\
\mathbf{u} \\
\mathbf{u} \\
\mathbf{u} \\
\mathbf{u} \\
\mathbf{u} \\
\mathbf{u} \\
\mathbf{u} \\
\mathbf{u} \\
\mathbf{u} \\
\mathbf{u} \\
\mathbf{u} \\
\mathbf{u} \\
\mathbf{u}\end{array}$ & $\begin{array}{c}10.000 \\
10.000 \\
10.000 \\
10.000 \\
26.000 \\
19.000 \\
5.000 \\
5.000 \\
5.000 \\
5.000 \\
5.000 \\
5.000 \\
10.000 \\
5.000 \\
5.000 \\
10.000 \\
5.000 \\
5.000 \\
5.000 \\
5.000 \\
5.000 \\
5.000 \\
5.000 \\
5.000 \\
H / R \\
5.000 \\
10.000 \\
10.000 \\
5.000 \\
5.000 \\
5.000 \\
5.000 \\
5.000 \\
5.000 \\
5.000 \\
H / R \\
H / R \\
\end{array}$ & $\begin{array}{c}\mathbf{u} \\
\mathbf{u} \\
\mathbf{u} \\
\mathbf{u} \\
\mathbf{u} \\
\mathbf{u} \\
\mathbf{u} \\
\mathbf{u} \\
\mathbf{u} \\
\mathbf{u} \\
\mathbf{u} \\
\mathbf{u} \\
\mathbf{u} \\
\mathbf{u} \\
\mathbf{u} \\
\mathbf{u} \\
\mathbf{u} \\
\mathbf{u} \\
\mathbf{u} \\
\mathbf{u} \\
\mathbf{u} \\
\mathbf{u} \\
\mathbf{u} \\
\mathbf{u} \\
\mathbf{u} \\
\mathbf{u} \\
\mathbf{u} \\
\mathbf{u} \\
\mathbf{u} \\
\mathbf{u} \\
\mathbf{u} \\
\mathbf{u} \\
\mathbf{u} \\
\mathbf{u}\end{array}$ & $\begin{array}{c}10.000 \\
10.000 \\
10.000 \\
10.000 \\
47.000 \\
47.000 \\
5.000 \\
5.000 \\
5.000 \\
5.000 \\
5.000 \\
5.000 \\
10.000 \\
5.000 \\
5.000 \\
10.000 \\
5.000 \\
5.000 \\
5.000 \\
5.000 \\
5.000 \\
5.000 \\
5.000 \\
5.000 \\
H / R \\
5.000 \\
10.000 \\
10.000 \\
5.000 \\
5.000 \\
5.000 \\
5.000 \\
5.000 \\
5.000 \\
5.000 \\
41 / R \\
11 / R \\
\end{array}$ & $\begin{array}{c}u \\
u \\
u \\
u \\
u \\
\mathbf{u} \\
\mathbf{u} \\
\mathbf{u} \\
\mathbf{u} \\
\mathbf{u} \\
\mathbf{u} \\
\mathbf{u} \\
\mathbf{u} \\
\mathbf{u} \\
\mathbf{u} \\
\mathbf{u} \\
\mathbf{u} \\
\mathbf{u} \\
\mathbf{u} \\
\mathbf{u} \\
\mathbf{u} \\
\mathbf{u} \\
\mathbf{u} \\
\mathbf{u} \\
\mathbf{u} \\
\mathbf{u} \\
\mathbf{u} \\
\mathbf{u} \\
\mathbf{u} \\
\mathbf{u} \\
\mathbf{u} \\
\mathbf{u} \\
\mathbf{u} \\
\mathbf{u}\end{array}$ & $\begin{array}{c}11.000 \\
11.000 \\
11.000 \\
11.000 \\
45.000 \\
42.000 \\
5.000 \\
5.000 \\
5.000 \\
5.000 \\
5.000 \\
5.000 \\
11.000 \\
5.000 \\
5.000 \\
11.000 \\
5.000 \\
5.000 \\
5.000 \\
5.000 \\
5.000 \\
5.000 \\
5.000 \\
5.000 \\
11 / R \\
5.000 \\
11.000 \\
11.000 \\
5.000 \\
5.000 \\
2.000 \\
5.000 \\
5.000 \\
5.000 \\
5.000 \\
\\
11 / R \\
11 / R\end{array}$ & $\begin{array}{c}u \\
u \\
u \\
u \\
u \\
u \\
u \\
u \\
u \\
u \\
u \\
u \\
u \\
u \\
u \\
v \\
u \\
u \\
u \\
u \\
u \\
u \\
u \\
u \\
u \\
u \\
u \\
u \\
u \\
u \\
u \\
u \\
u \\
u\end{array}$ & $\begin{array}{c}11.000 \\
11.000 \\
11.000 \\
11.000 \\
45.000 \\
51.000 \\
6.000 \\
5.000 \\
5.000 \\
5.000 \\
2.000 \\
5.000 \\
11.000 \\
5.000 \\
5.000 \\
11.000 \\
5.000 \\
5.000 \\
5.000 \\
5.000 \\
5.000 \\
5.000 \\
5.000 \\
5.000 \\
N / R \\
5.000 \\
11.000 \\
11.000 \\
5.000 \\
5.000 \\
4.000 \\
5.000 \\
5.000 \\
5.000 \\
5.000 \\
11 / R \\
1 / R\end{array}$ & 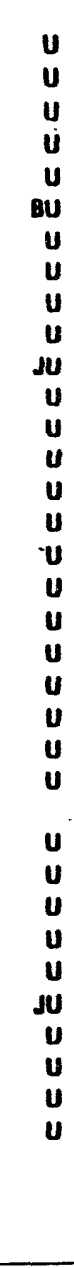 \\
\hline
\end{tabular}


Location 307 T-1, 399-3-15

\begin{tabular}{|c|c|c|c|c|c|c|c|c|c|c|c|c|c|}
\hline \multirow[t]{2}{*}{ Parameter } & \multirow{2}{*}{$\begin{array}{l}\text { Sampl } \\
\text { Depth } \\
\text { Units } \\
\end{array}$} & \multicolumn{2}{|c|}{$\begin{array}{r}\text { 801CF9 } \\
4.60\end{array}$} & \multicolumn{2}{|c|}{$\begin{array}{r}\text { BO1CGO } \\
7.00\end{array}$} & \multicolumn{2}{|c|}{$\begin{array}{r}801 C G 2 \\
9.00\end{array}$} & \multicolumn{2}{|c|}{$\begin{array}{r}801 C G 4 \\
11.60\end{array}$} & \multicolumn{2}{|c|}{$\begin{array}{r}801 C 65 \\
13.60\end{array}$} & \multicolumn{2}{|c|}{$\begin{array}{r}801 C 66 \\
15.00\end{array}$} \\
\hline & & Result & $\mathbf{a}$ & Result & a & Result & $a$ & Result & $\mathbf{a}$ & Result & $a$ & Result & $a$ \\
\hline 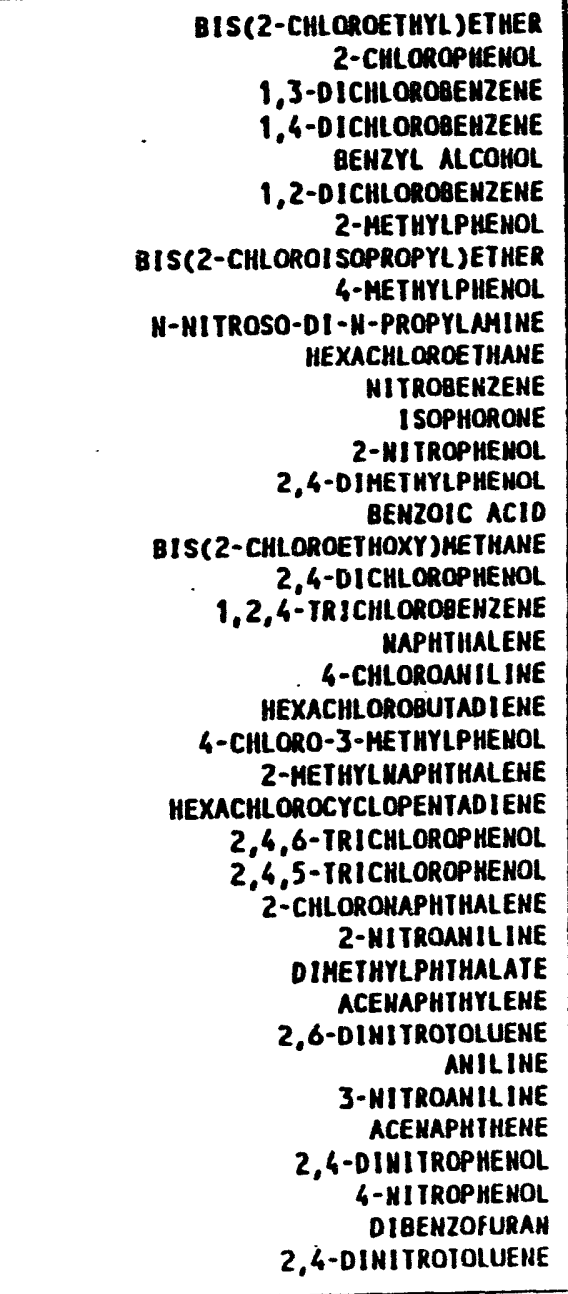 & & 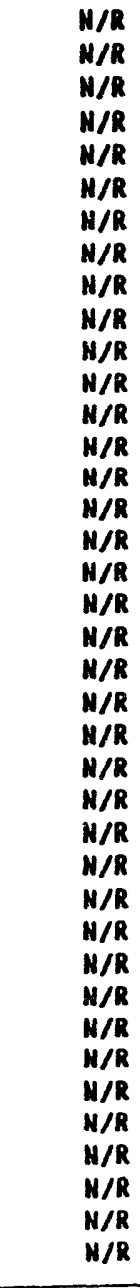 & & 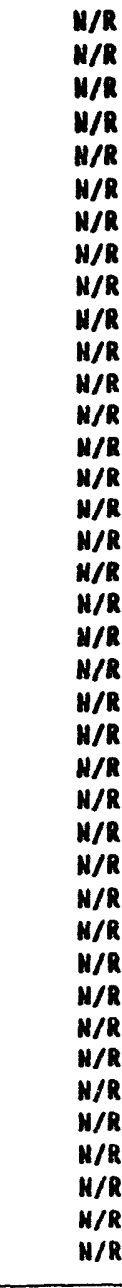 & & 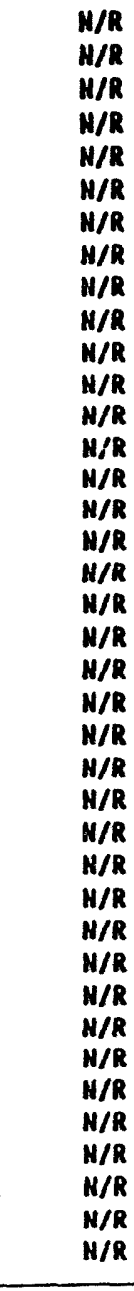 & & 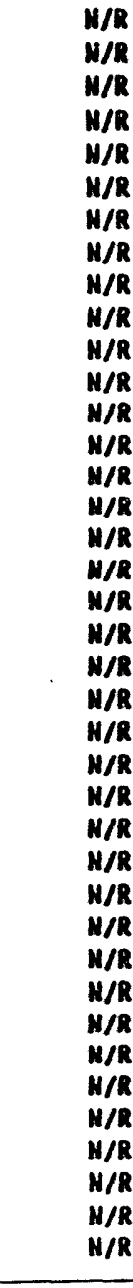 & & 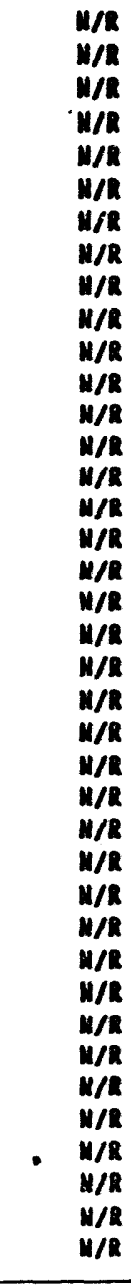 & & 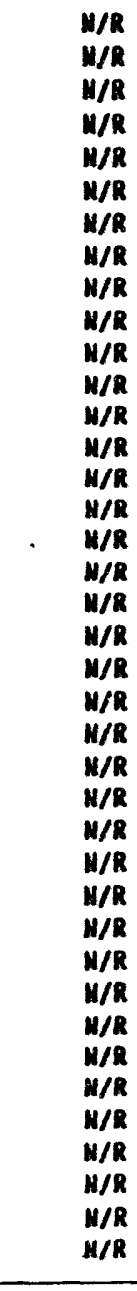 & \\
\hline
\end{tabular}


Locetton 307 T-1, 399-3-15

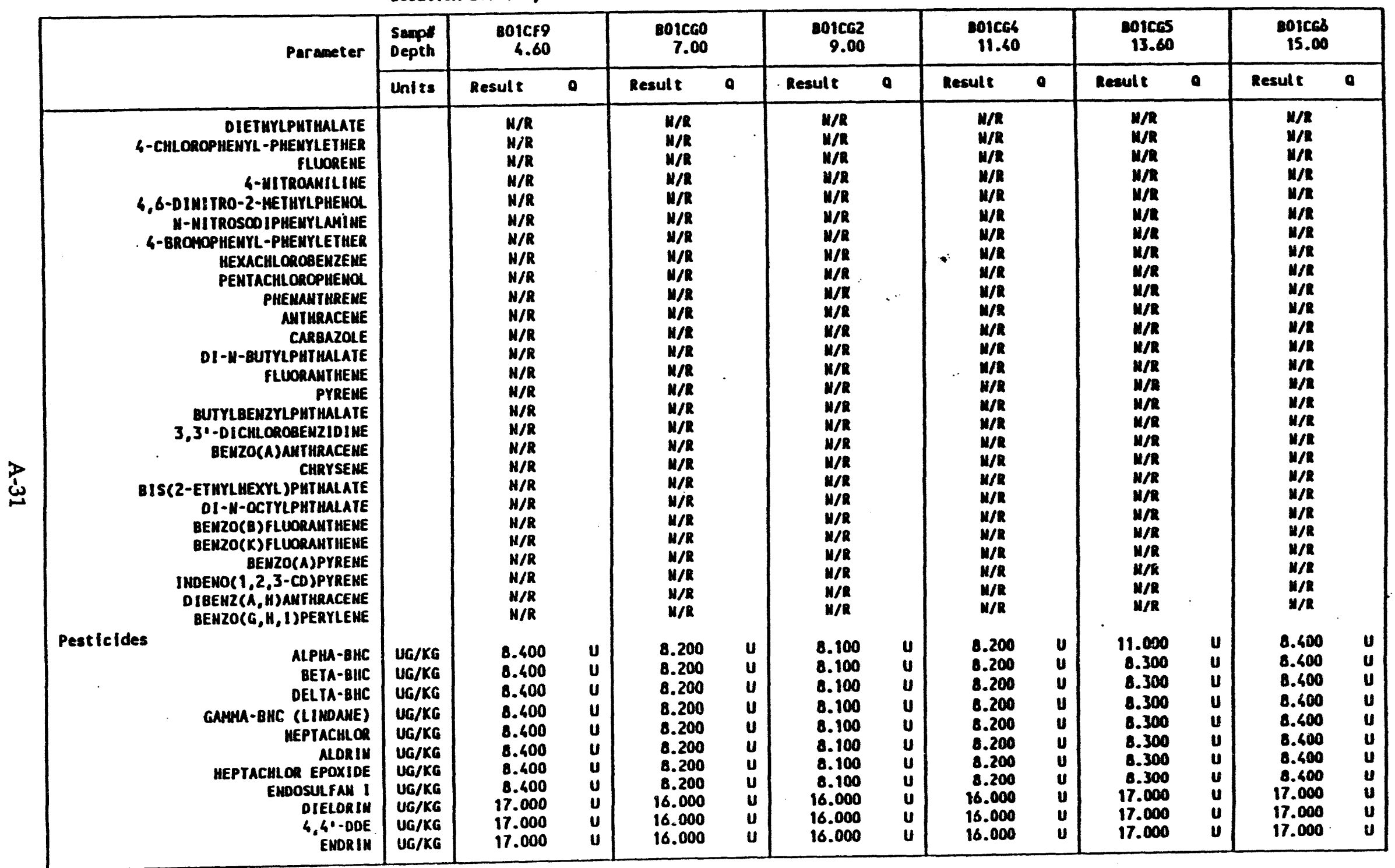


Lacation 307 1-1, 399-3-15

\begin{tabular}{|c|c|c|c|c|c|c|c|c|c|c|c|c|c|}
\hline \multirow[t]{2}{*}{ Paraceter } & \multirow{2}{*}{ 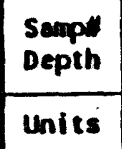 } & \multicolumn{2}{|l|}{$\begin{array}{r}\text { B01CF9 } \\
4.60\end{array}$} & \multicolumn{2}{|l|}{$\begin{array}{r}\text { Boicco } \\
7.00\end{array}$} & \multicolumn{2}{|l|}{$\begin{array}{r}001 C 62 \\
9.00\end{array}$} & \multicolumn{2}{|c|}{$\begin{array}{l}801 c 64 \\
11.40\end{array}$} & \multicolumn{2}{|l|}{$\begin{array}{r}001065 \\
13.60\end{array}$} & \multicolumn{2}{|l|}{$\begin{array}{r}201 c 66 \\
15.00\end{array}$} \\
\hline & & Result & $a$ & Result & a & Result & a & Result & 0 & Result & $a$ & Result & a \\
\hline 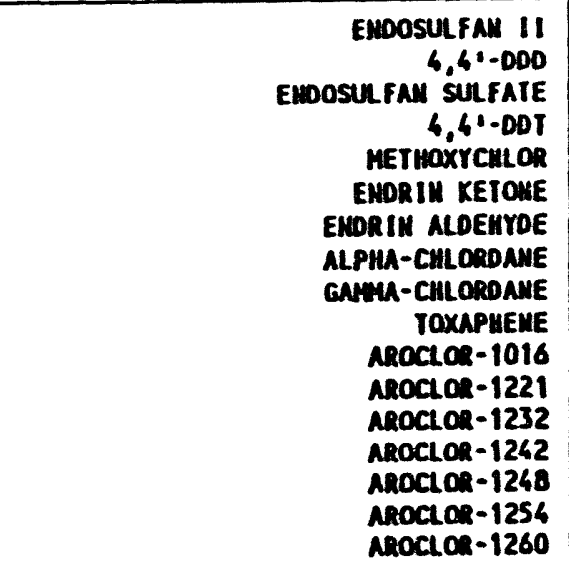 & $\begin{array}{l}\text { UG/KG } \\
\text { UG/KG } \\
\text { UG/KG } \\
\text { UG/KG } \\
\text { UG/KG } \\
\text { UG/KG } \\
\text { UG/KG } \\
\text { UG/KG } \\
\text { UG/KG } \\
\text { UG/KG } \\
\text { UG/KG } \\
\text { UG/KG } \\
\text { UG/KG } \\
\text { UG/KG } \\
\text { UG/KG } \\
\text { UG/KG }\end{array}$ & $\begin{array}{c}17.000 \\
17.000 \\
17.000 \\
17.000 \\
84.000 \\
17.000 \\
1 / R \\
84.000 \\
84.000 \\
170.000 \\
84.000 \\
84.000 \\
84.000 \\
84.000 \\
84.000 \\
170.000 \\
170.000\end{array}$ & $\begin{array}{l}u \\
u \\
u \\
u \\
u \\
u \\
u \\
u \\
u \\
u \\
u \\
u \\
u \\
u \\
u \\
u\end{array}$ & $\begin{array}{c}16.000 \\
16.000 \\
16.000 \\
16.000 \\
82.000 \\
16.000 \\
11 / R \\
82.000 \\
82.000 \\
160.000 \\
82.000 \\
82.000 \\
82.000 \\
82.000 \\
82.000 \\
160.000 \\
160.000\end{array}$ & $\begin{array}{l}u \\
u \\
u \\
u \\
u \\
u \\
u \\
u \\
u \\
u \\
u \\
u \\
u \\
u \\
u \\
u\end{array}$ & $\begin{array}{c}16.000 \\
16.000 \\
16.000 \\
16.000 \\
81.000 \\
16.000 \\
11 / R \\
81.000 \\
81.000 \\
160.000 \\
81.000 \\
81.000 \\
81.000 \\
81.000 \\
81.000 \\
110.000 \\
50.000\end{array}$ & $\begin{array}{l}u \\
u \\
u \\
u \\
u \\
u \\
u \\
u \\
u \\
u \\
u \\
u \\
u \\
u \\
d \\
d\end{array}$ & $\begin{array}{r}16.000 \\
16.000 \\
16.000 \\
16.000 \\
82.000 \\
16.000 \\
11 / 1 \\
82.000 \\
82.000 \\
160.000 \\
82.000 \\
82.000 \\
82.000 \\
82.000 \\
82.000 \\
160.000 \\
160.000\end{array}$ & $\begin{array}{l}u \\
u \\
u \\
u \\
u \\
u \\
u \\
u \\
u \\
u \\
u \\
u \\
u \\
u \\
u \\
u\end{array}$ & $\begin{array}{r}17.000 \\
17.000 \\
17.000 \\
17.000 \\
83.000 \\
17.000 \\
171 \\
83.000 \\
83.000 \\
170.000 \\
83.000 \\
83.000 \\
83.000 \\
83.000 \\
83.000 \\
170.000 \\
170.000\end{array}$ & $\begin{array}{l}u \\
u \\
u \\
u \\
u \\
u \\
u \\
u \\
u \\
u \\
u \\
u \\
u \\
u \\
u \\
u\end{array}$ & $\begin{array}{c}17.000 \\
17.000 \\
17.000 \\
17.000 \\
84.000 \\
17.000 \\
11 / 1 \\
84.000 \\
84.000 \\
170.000 \\
84.000 \\
84.000 \\
64.000 \\
86.000 \\
84.000 \\
170.000 \\
170.000\end{array}$ & $\begin{array}{l}u \\
u \\
u \\
u \\
u \\
u \\
u \\
u \\
u \\
u \\
u \\
u \\
u \\
u \\
u \\
u\end{array}$ \\
\hline
\end{tabular}


Location 307 T-1, 399-3-15

\begin{tabular}{|c|c|c|c|c|c|c|c|c|c|c|c|c|c|}
\hline \multirow[t]{2}{*}{ Parmeter } & \multirow{2}{*}{$\begin{array}{l}\text { Sampl } \\
\text { Depth }\end{array}$} & \multicolumn{2}{|l|}{$\begin{array}{r}801 C G 7 \\
17.00\end{array}$} & \multicolumn{2}{|l|}{$\begin{array}{r}801 C \mathrm{CG} \\
20.00\end{array}$} & \multicolumn{2}{|l|}{$\begin{array}{r}01069 \\
22.50\end{array}$} & \multicolumn{2}{|l|}{$\begin{array}{r}010100 \\
26.50\end{array}$} & \multicolumn{2}{|c|}{$\begin{array}{c}\text { Bo1cuoph } \\
26.50\end{array}$} & \multicolumn{2}{|l|}{$\begin{array}{l}901 \mathrm{cul} \\
30.00\end{array}$} \\
\hline & & Result & $a$ & Result & $\mathbf{a}$ & Result & a & Result & $a$ & Result & a & Result & $a$ \\
\hline 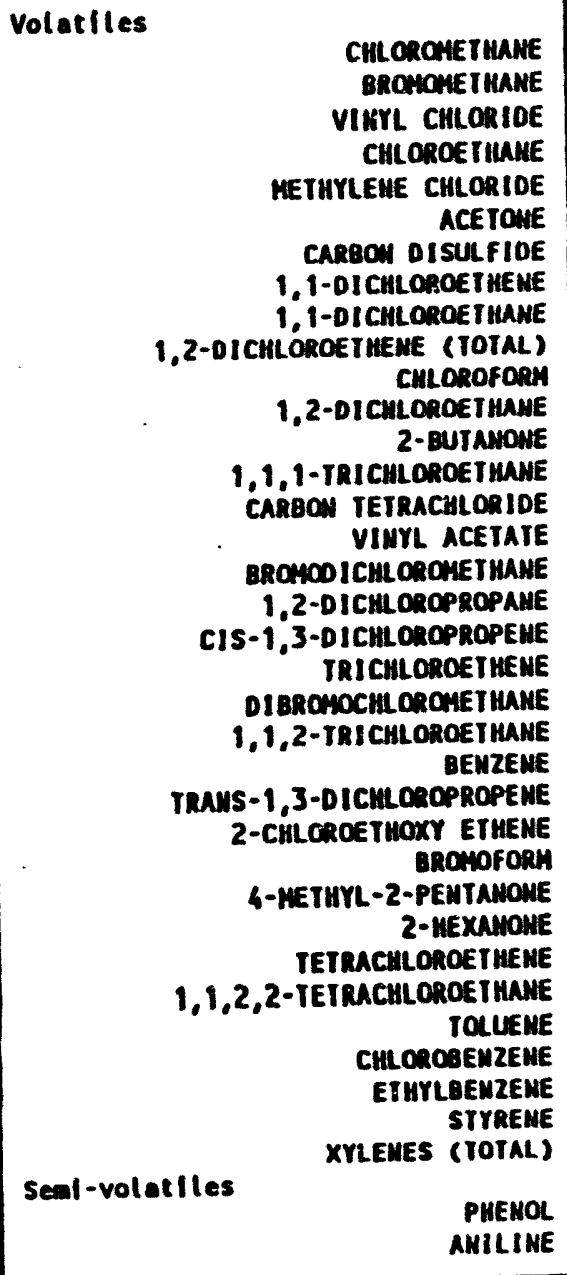 & $\begin{array}{l}U G / K G \\
U G / K G \\
U G / K G \\
U G / K G \\
U G / K G \\
U G / K G \\
U G / K G \\
U G / K G \\
U G / K G \\
U G / K G \\
U G / K G \\
U G / K G \\
U G / K G \\
U G / K G \\
U G / K G \\
U G / K G \\
U G / K G \\
U G / K G \\
U G / K G \\
U G / K G \\
U G / K G \\
U G / K G \\
U G / K G \\
U G / K G \\
U G / K G \\
U G / K G \\
U G / K G \\
U G / K G \\
U G / K G \\
U G / K G \\
U G / K G \\
U G / K G \\
U G / K G \\
U G / K G\end{array}$ & $\begin{array}{c}10.000 \\
10.000 \\
10.000 \\
10.000 \\
67.000 \\
73.000 \\
2.000 \\
5.000 \\
5.000 \\
5.000 \\
2.000 \\
5.000 \\
10.000 \\
5.000 \\
5.000 \\
10.000 \\
5.000 \\
5.000 \\
5.000 \\
5.000 \\
5.000 \\
5.000 \\
5.000 \\
5.000 \\
11 / R \\
5.000 \\
10.000 \\
10.000 \\
5.000 \\
5.000 \\
2.000 \\
5.000 \\
5.000 \\
5.000 \\
5.000 \\
\\
11 / 2 \\
11 / R\end{array}$ & $\begin{array}{r}\mathbf{U} \\
\mathbf{U} \\
\mathbf{U} \\
\mathbf{U} \\
\mathbf{u} \\
\mathbf{B U} \\
\mathbf{J U} \\
\mathbf{U} \\
\mathbf{U} \\
\mathbf{U} \\
\mathbf{U} \\
\mathbf{U} \\
\mathbf{U} \\
\mathbf{U} \\
\mathbf{U} \\
\mathbf{U} \\
\mathbf{U} \\
\mathbf{U} \\
\mathbf{U} \\
\mathbf{U} \\
\mathbf{U} \\
\mathbf{U} \\
\mathbf{U} \\
\mathbf{U} \\
\mathbf{U} \\
\mathbf{U} \\
\mathbf{U} \\
\mathbf{U} \\
\mathbf{U} \\
\mathbf{U} \\
\mathbf{U} \\
\mathbf{U} \\
\mathbf{U} \\
\mathbf{U}\end{array}$ & $\begin{array}{r}11.000 \\
11.000 \\
11.000 \\
11.000 \\
5.000 \\
21.000 \\
5.000 \\
5.000 \\
5.000 \\
5.000 \\
5.000 \\
5.000 \\
11.000 \\
5.000 \\
5.000 \\
11.000 \\
5.000 \\
5.000 \\
5.000 \\
5.000 \\
5.000 \\
5.000 \\
5.000 \\
5.000 \\
11 / 1 \\
5.000 \\
11.000 \\
11.000 \\
5.000 \\
5.000 \\
5.000 \\
5.000 \\
5.000 \\
5.000 \\
5.000 \\
\\
11 / 2 \\
11 / R\end{array}$ & $\begin{array}{l}\mathbf{u} \\
\mathbf{u} \\
\mathbf{u} \\
\mathbf{u} \\
\mathbf{u} \\
\mathbf{u} \\
\mathbf{u} \\
\mathbf{u} \\
\mathbf{u} \\
\mathbf{u} \\
\mathbf{u} \\
\mathbf{u} \\
\mathbf{u} \\
\mathbf{u} \\
\mathbf{u} \\
\mathbf{u} \\
\mathbf{u} \\
\mathbf{u} \\
\mathbf{u} \\
\mathbf{U} \\
\mathbf{u} \\
\mathbf{u} \\
\mathbf{U} \\
\mathbf{u} \\
\mathbf{u} \\
\mathbf{U} \\
\mathbf{U} \\
\mathbf{u} \\
\mathbf{u} \\
\mathbf{u} \\
\mathbf{u} \\
\mathbf{u} \\
\mathbf{u}\end{array}$ & $\begin{array}{c}10.000 \\
10.000 \\
10.000 \\
10.000 \\
39.000 \\
40.000 \\
5.000 \\
5.000 \\
5.000 \\
5.000 \\
2.000 \\
5.000 \\
10.000 \\
5.000 \\
5.000 \\
10.000 \\
5.000 \\
5.000 \\
5.000 \\
5.000 \\
5.000 \\
5.000 \\
5.000 \\
5.000 \\
11 / R \\
5.000 \\
10.000 \\
10.000 \\
5.000 \\
5.000 \\
5.000 \\
5.000 \\
5.000 \\
5.000 \\
5.000 \\
\\
11 / 2 \\
11 / R\end{array}$ & $\begin{array}{c}u \\
u \\
u \\
u \\
u \\
u \\
u \\
u \\
u \\
u \\
u \\
u \\
u \\
u \\
u \\
u \\
u \\
u \\
u \\
u \\
u \\
u \\
u \\
u \\
u \\
u \\
u \\
u \\
u \\
u \\
u \\
u \\
u \\
u\end{array}$ & $\begin{array}{c}11.000 \\
11.000 \\
11.000 \\
11.000 \\
42.000 \\
62.000 \\
5.000 \\
5.000 \\
5.000 \\
5.000 \\
5.000 \\
5.000 \\
11.000 \\
5.000 \\
5.000 \\
11.000 \\
5.000 \\
5.000 \\
5.000 \\
5.000 \\
5.000 \\
5.000 \\
5.000 \\
5.000 \\
11 / R \\
5.000 \\
11.000 \\
11.000 \\
6.000 \\
5.000 \\
3.000 \\
5.000 \\
5.000 \\
5.000 \\
5.000 \\
\\
11 / R \\
11 / R\end{array}$ & $\begin{array}{c}u \\
u \\
u \\
u \\
u \\
u \\
u \\
u \\
u \\
u \\
u \\
u \\
u \\
u \\
u \\
u \\
u \\
u \\
u \\
u \\
u \\
u \\
u \\
u \\
u \\
u \\
u \\
u \\
u \\
u \\
u \\
u \\
u \\
u\end{array}$ & 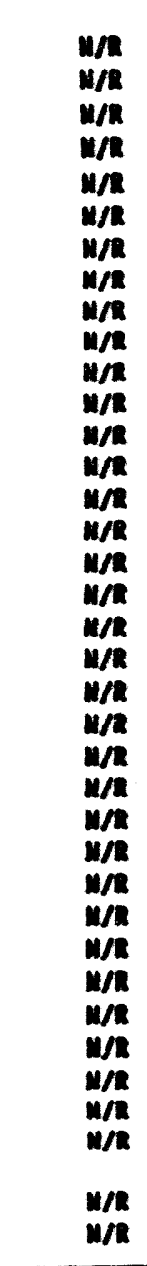 & & $\begin{array}{c}11.000 \\
11.000 \\
11.000 \\
11.000 \\
21.000 \\
35.000 \\
5.000 \\
5.000 \\
5.000 \\
5.000 \\
5.000 \\
5.000 \\
11.000 \\
5.000 \\
5.000 \\
11.000 \\
5.000 \\
5.000 \\
5.000 \\
5.000 \\
5.000 \\
5.000 \\
5.000 \\
5.000 \\
11 / 2 \\
5.000 \\
11.000 \\
11.000 \\
5.000 \\
5.000 \\
5.000 \\
5.000 \\
5.000 \\
5.000 \\
5.000 \\
\\
11 / 1 \\
11 / 1 \\
\end{array}$ & $\begin{array}{l}u \\
u \\
u \\
u \\
u \\
u \\
u \\
u \\
u \\
u \\
u \\
u \\
u \\
u \\
u \\
u \\
u \\
u \\
u \\
u \\
u \\
u \\
u \\
u \\
u \\
u \\
u \\
u \\
u \\
u \\
u \\
u \\
u \\
u\end{array}$ \\
\hline
\end{tabular}


Location 307 r-1, 399-3-15

\begin{tabular}{|c|c|c|c|c|c|c|c|c|c|c|c|c|c|}
\hline \multirow[t]{2}{*}{ Parameter } & \multirow{2}{*}{$\begin{array}{l}\text { Semp: } \\
\text { Depth } \\
\text { Unlts }\end{array}$} & \multicolumn{2}{|l|}{$\begin{array}{l}\text { 801CG7 } \\
17.00\end{array}$} & \multicolumn{2}{|l|}{$\begin{array}{r}101 C 68 \\
20.00\end{array}$} & \multicolumn{2}{|l|}{$\begin{array}{r}0.1069 \\
22.50\end{array}$} & \multicolumn{2}{|l|}{$\begin{array}{r}001 \text { CHO } \\
24.50\end{array}$} & \multicolumn{2}{|c|}{$\begin{array}{c}801 \mathrm{cmoph} \\
26.50\end{array}$} & \multicolumn{2}{|l|}{$\begin{array}{r}0.01 c u 1 \\
30.00\end{array}$} \\
\hline & & Result & 0 & Result & $a$ & Result & $a$ & Result & $a$ & Result & 0 & Result & $a$ \\
\hline 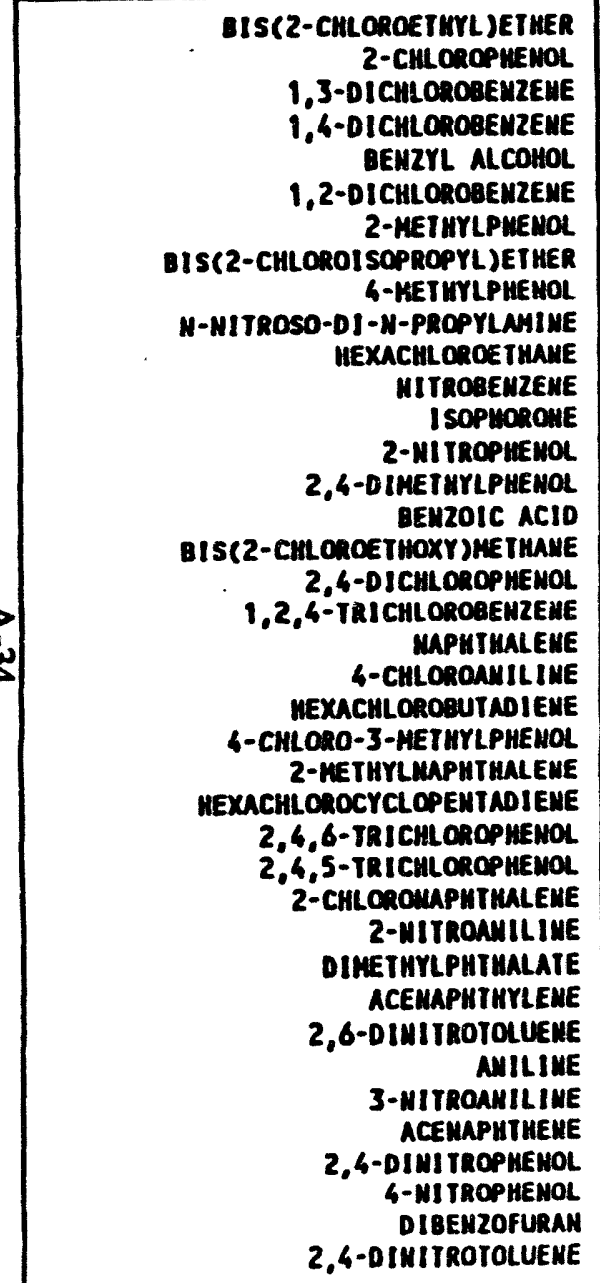 & & $\begin{array}{l}W / R \\
M / R \\
M / R \\
M / R \\
M / R \\
M / R \\
M / R \\
M / R \\
N / R \\
M / R \\
M / R \\
M / R \\
M / R \\
M / R \\
M / R \\
M / R \\
M / R \\
M / R \\
M / R \\
M / R \\
M / R \\
M / R \\
M / R \\
M / R \\
M / R \\
M / R \\
M / R \\
M / R \\
M / R \\
M / R \\
M / R \\
M / R \\
M / R \\
M / R \\
M / R \\
M / R \\
M / R \\
M / R \\
M / R \\
M / R \\
M / R\end{array}$ & & 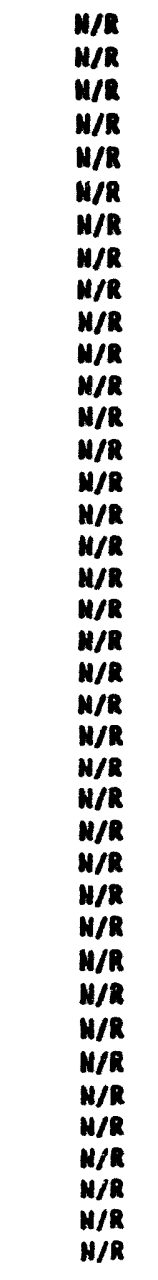 & & 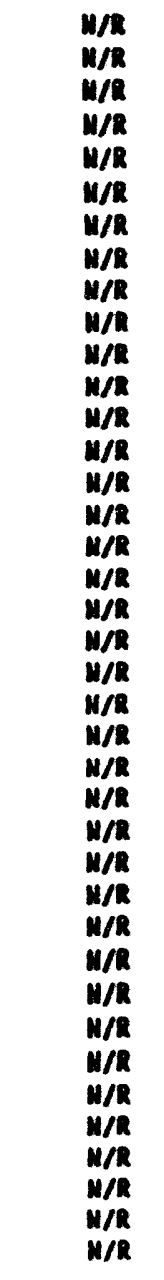 & & 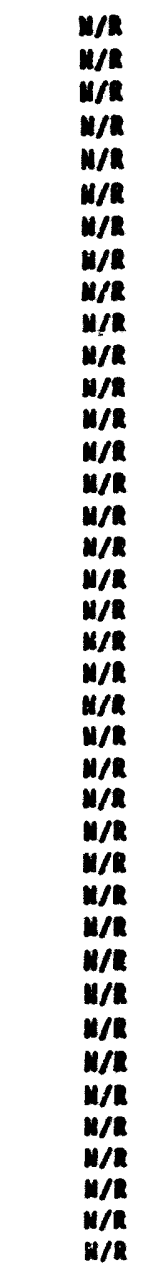 & & 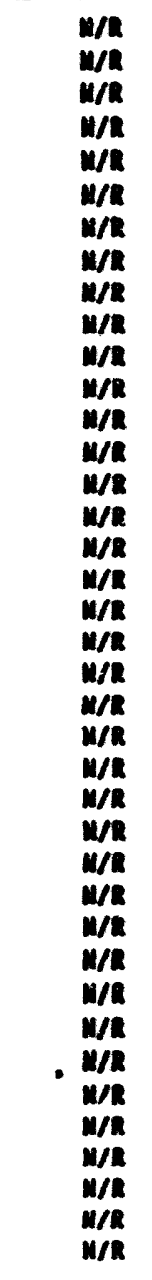 & & 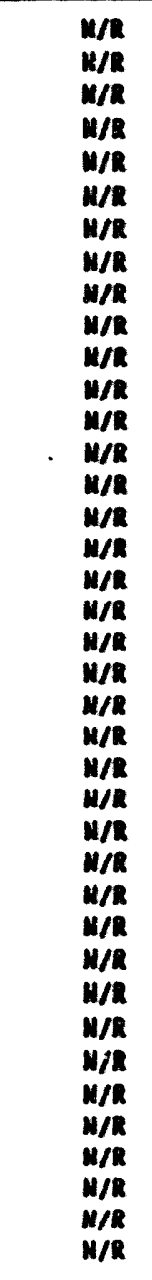 & \\
\hline
\end{tabular}


Locotion 307 1-1, 399-3-15

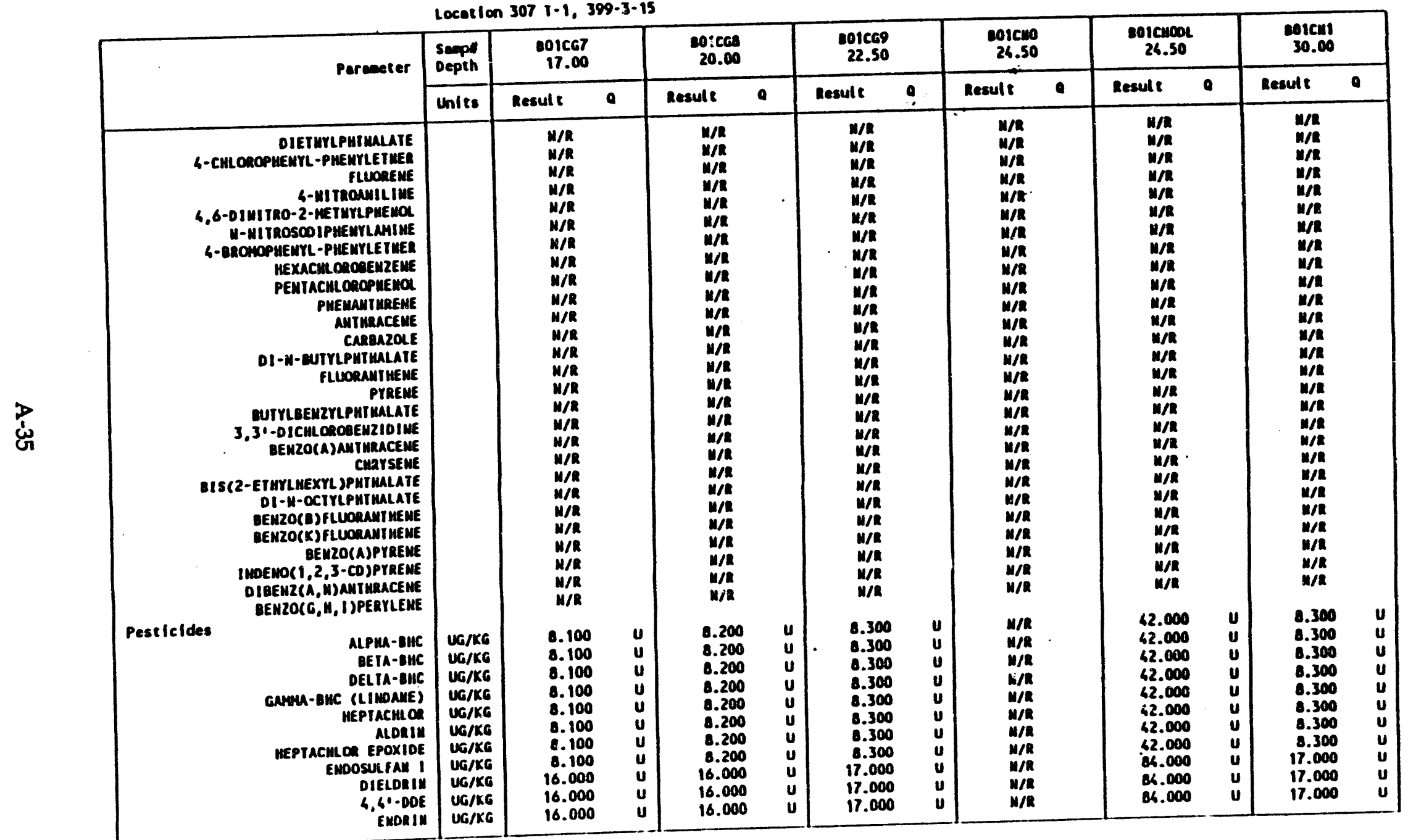


Locat Ion 307 Y-1, 399-3-15

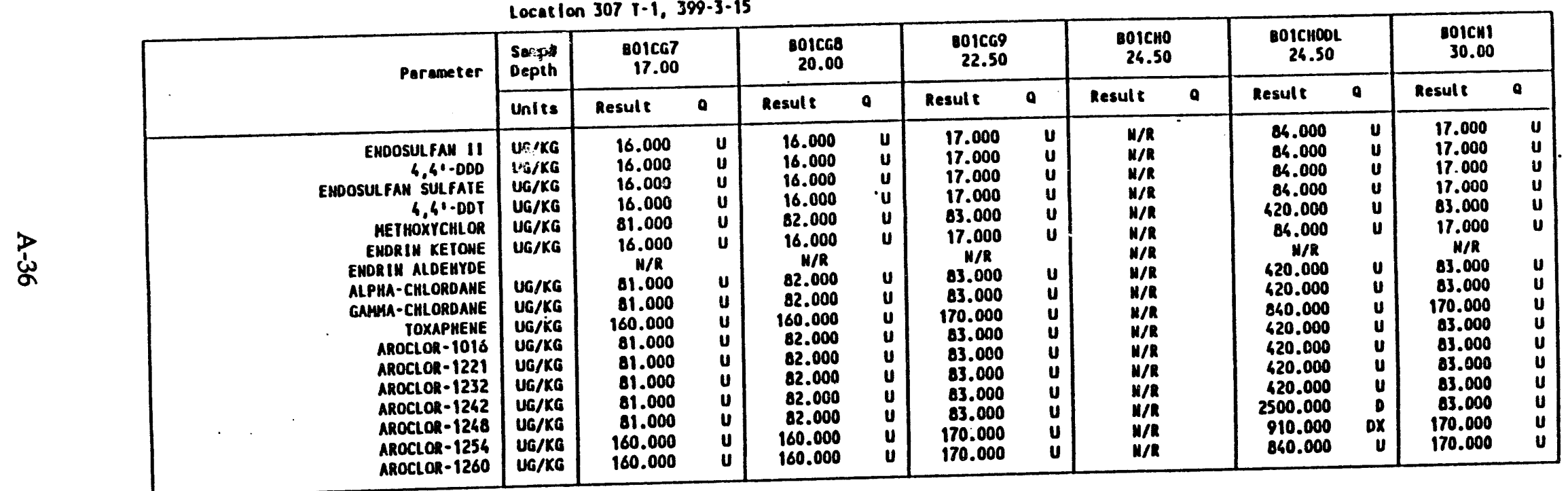


Location 307 T-1, 399-3-15

\begin{tabular}{|c|c|c|c|c|c|c|c|c|c|c|c|c|c|}
\hline \multirow[t]{2}{*}{ Parameter } & \multirow{2}{*}{$\frac{\begin{array}{c}\text { Sampl } \\
\text { Depth }\end{array}}{\text { Units }}$} & \multicolumn{2}{|l|}{$\begin{array}{r}\text { B01CH2 } \\
37.00\end{array}$} & \multicolumn{2}{|l|}{$\begin{array}{r}\text { BO1RJO } \\
62.00\end{array}$} & \multicolumn{2}{|l|}{$\begin{array}{r}801 R J 1 \\
47.00\end{array}$} & \multicolumn{2}{|l|}{$\begin{array}{r}801 R J 2 \\
53.00\end{array}$} & \multicolumn{2}{|l|}{$\begin{array}{r}801 R . J 3 \\
58.00\end{array}$} & \multicolumn{2}{|c|}{$\begin{array}{c}\text { S01RJ3RE } \\
58.00\end{array}$} \\
\hline & & Result & a & Result & 9 & , Result & a & Result & a & Result & 0 & Result & 9 \\
\hline 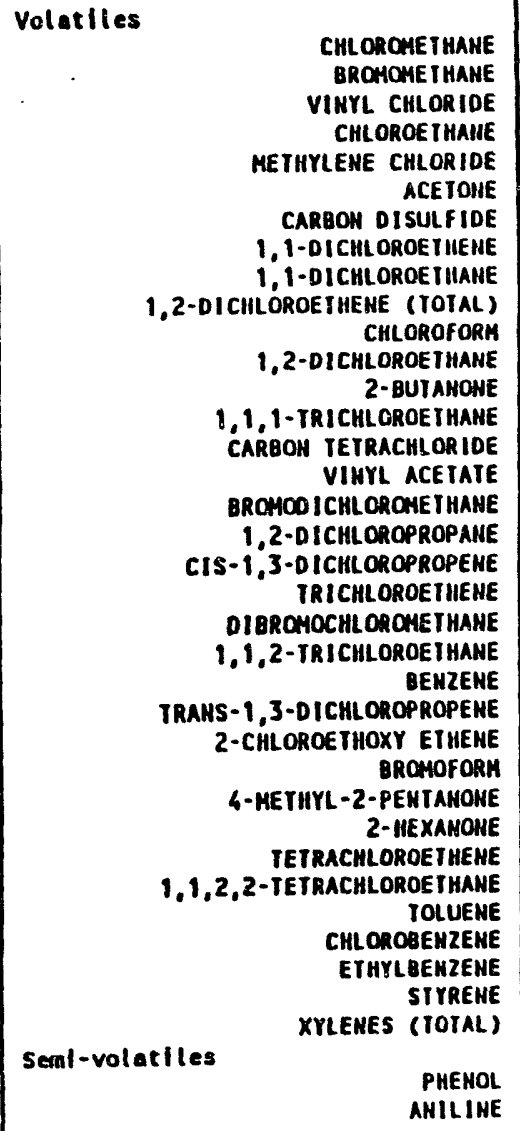 & $\begin{array}{l}U G / K G \\
U G / K G \\
U G / K G \\
U G / K G \\
U G / K G \\
U G / K G \\
U G / K G \\
U G / K G \\
U G / K G \\
U G / K G \\
U G / K G \\
U G / K G \\
U G / K G \\
U G / K G \\
U G / K G \\
U G / K G \\
U G / K G \\
U G / K G \\
U G / K G \\
U G / K G \\
U G / K G \\
U G / K G \\
U G / K G \\
U G / K G \\
U G / K G \\
U G / K G \\
U G / K G \\
U G / K G \\
U G / K G \\
U G / K G \\
U G / K G \\
U G / K G \\
U G / K G \\
U G / K G\end{array}$ & $\begin{array}{r}11.000 \\
11.000 \\
11.000 \\
11.000 \\
21.000 \\
43.000 \\
5.000 \\
5.000 \\
5.000 \\
5.000 \\
5.000 \\
5.000 \\
11.000 \\
5.000 \\
5.000 \\
11.000 \\
5.000 \\
5.000 \\
5.000 \\
5.000 \\
5.000 \\
5.000 \\
5.000 \\
5.000 \\
H / R \\
5.000 \\
11.000 \\
11.000 \\
5.000 \\
5.000 \\
5.000 \\
5.000 \\
5.000 \\
5.000 \\
5.000 \\
H / R \\
H / R \\
H / R\end{array}$ & $\begin{array}{c}u \\
u \\
u \\
u \\
u \\
u \\
u \\
u \\
u \\
u \\
u \\
u \\
u \\
u \\
u \\
u \\
u \\
u \\
u \\
u \\
u \\
u \\
u \\
u \\
u \\
u \\
u \\
u \\
u \\
u \\
u \\
u \\
u \\
u \\
u\end{array}$ & $\begin{array}{r}11.000 \\
11.000 \\
11.000 \\
11.000 \\
14.000 \\
23.000 \\
5.000 \\
5.000 \\
5.000 \\
5.000 \\
5.000 \\
5.000 \\
11.000 \\
5.000 \\
5.000 \\
11.000 \\
5.000 \\
5.000 \\
5.000 \\
5.000 \\
5.000 \\
5.000 \\
5.000 \\
5.000 \\
11 / R \\
5.000 \\
11.000 \\
11.000 \\
5.000 \\
5.000 \\
5.000 \\
5.000 \\
5.000 \\
5.000 \\
5.000 \\
11 / R \\
11 / R\end{array}$ & 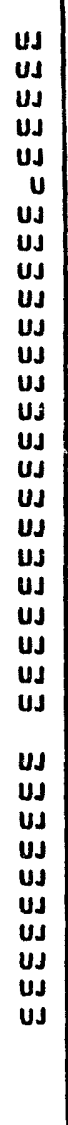 & $\begin{array}{r}11.000 \\
11.000 \\
11.000 \\
11.000 \\
11.000 \\
20.000 \\
5.000 \\
5.000 \\
5.000 \\
5.000 \\
5.000 \\
5.000 \\
11.000 \\
5.000 \\
5.000 \\
11.000 \\
5.000 \\
5.000 \\
5.000 \\
5.000 \\
5.000 \\
5.000 \\
5.000 \\
5.000 \\
11 / R \\
5.000 \\
11.000 \\
11.000 \\
5.000 \\
5.000 \\
5.000 \\
5.000 \\
5.000 \\
5.000 \\
5.000 \\
H / R \\
H / R \\
M / R\end{array}$ & 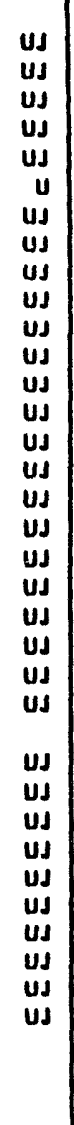 & $\begin{array}{r}13.000 \\
13.000 \\
13.000 \\
13.000 \\
18.000 \\
32.000 \\
6.000 \\
6.000 \\
6.000 \\
6.000 \\
6.000 \\
6.000 \\
13.000 \\
6.000 \\
6.000 \\
13.000 \\
6.000 \\
6.000 \\
6.000 \\
6.000 \\
6.000 \\
6.000 \\
6.000 \\
6.000 \\
H / R \\
6.000 \\
13.000 \\
13.000 \\
6.000 \\
6.000 \\
6.000 \\
6.000 \\
6.000 \\
6.000 \\
6.000 \\
H / R \\
H / R \\
H / R\end{array}$ & 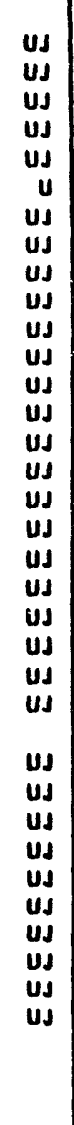 & $\begin{array}{r}11.000 \\
11.000 \\
11.000 \\
11.000 \\
7.000 \\
41.000 \\
6.000 \\
6.000 \\
6.000 \\
6.000 \\
6.000 \\
6.000 \\
11.000 \\
6.000 \\
6.000 \\
11.000 \\
6.000 \\
6.000 \\
6.000 \\
6.000 \\
6.000 \\
6.000 \\
6.000 \\
6.000 \\
11 / R \\
6.000 \\
11.000 \\
11.000 \\
6.000 \\
6.000 \\
6.000 \\
6.000 \\
6.000 \\
6.000 \\
6.000 \\
11 / R \\
11 / R\end{array}$ & 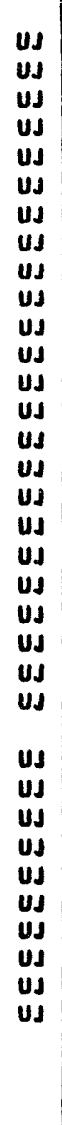 & $\begin{array}{r}11.000 \\
11.000 \\
11.000 \\
11.000 \\
13.000 \\
52.000 \\
6.000 \\
6.000 \\
6.000 \\
6.000 \\
6.000 \\
6.000 \\
11.000 \\
6.000 \\
6.000 \\
11.000 \\
6.000 \\
6.000 \\
6.000 \\
6.000 \\
6.000 \\
6.000 \\
6.000 \\
6.000 \\
11 / 8 \\
6.000 \\
11.000 \\
11.000 \\
6.000 \\
6.000 \\
6.000 \\
6.000 \\
6.000 \\
6.000 \\
6.000 \\
\\
11 / R \\
1 / R\end{array}$ & $\begin{array}{l}u \\
u \\
u \\
u \\
u \\
u \\
u \\
u \\
u \\
u \\
u \\
u \\
u \\
u \\
u \\
u \\
u \\
u \\
u \\
u \\
u \\
u \\
u \\
u \\
u \\
u \\
u \\
u \\
u \\
u \\
u \\
u \\
u \\
u \\
u \\
u\end{array}$ \\
\hline
\end{tabular}


WHC-SD-EN-TI-279, Rev. 0

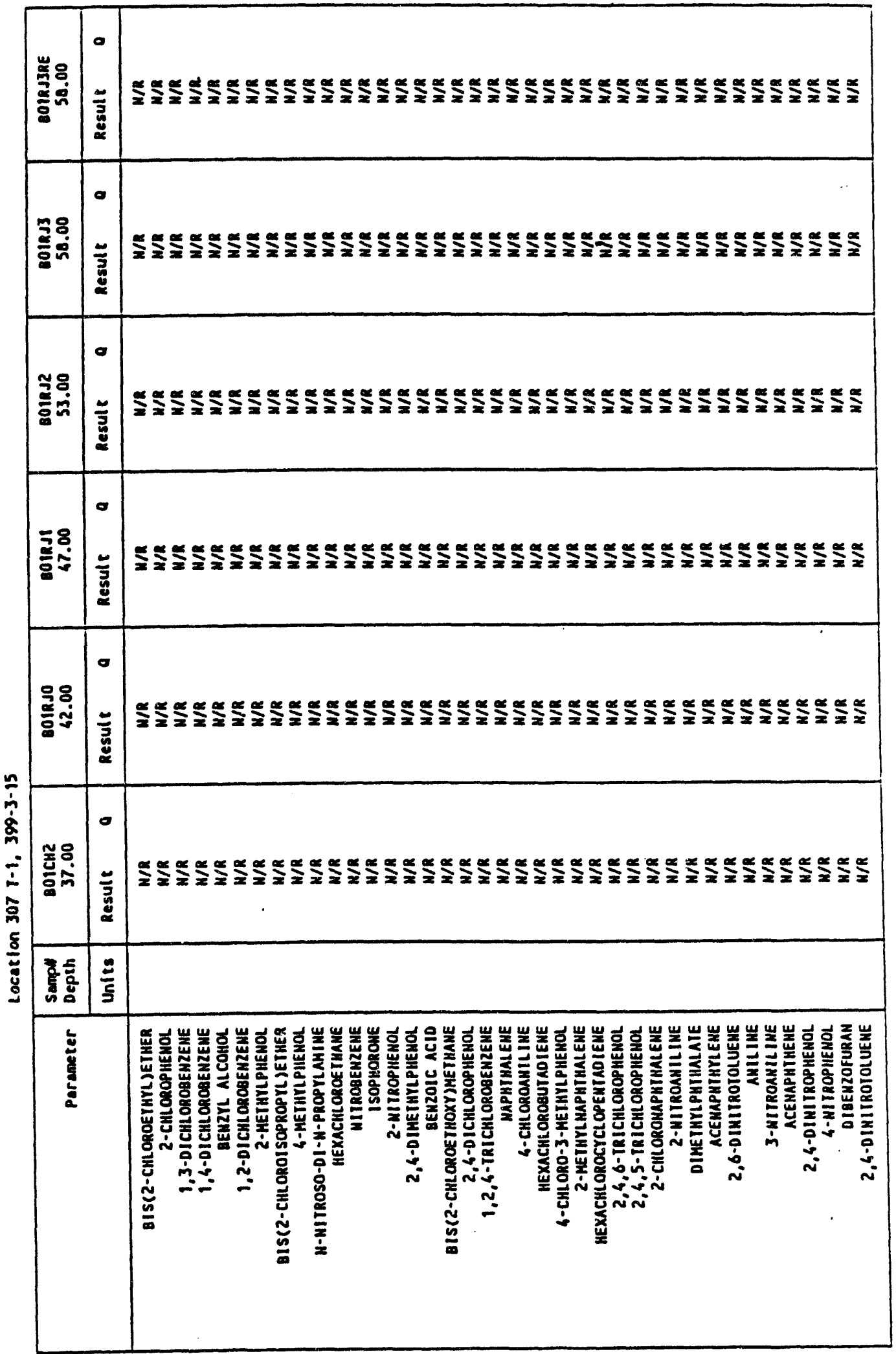


Locat ton 307 1-1, 399-3-15

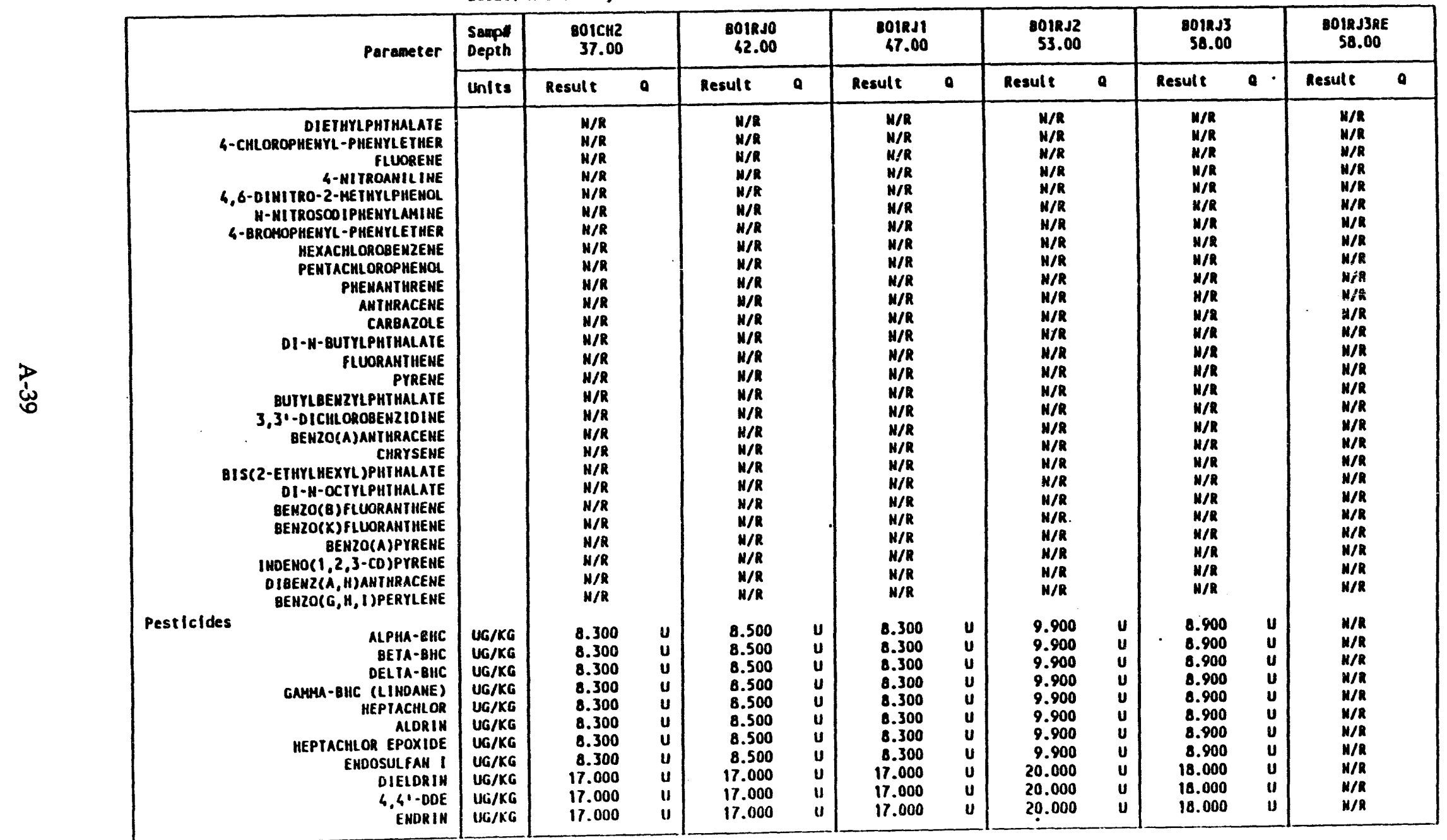




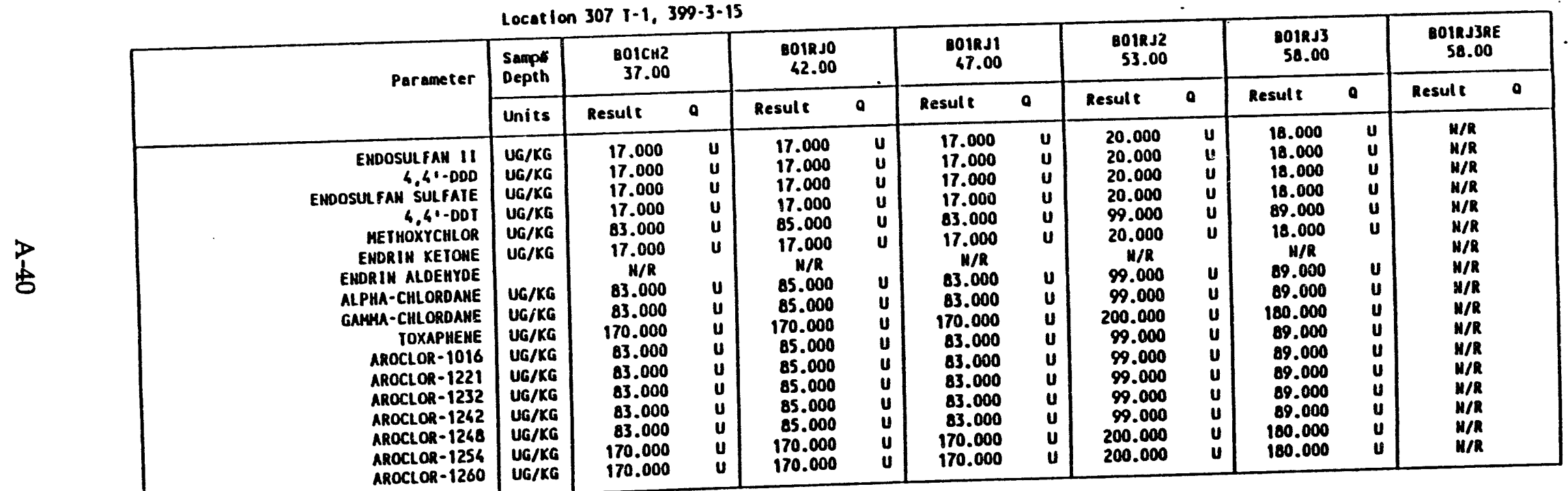


WHC-SD-EN-TI-279, Rev. 0

Location 307 T-1, 399-3-15

\begin{tabular}{|c|c|c|c|}
\hline \multirow[t]{2}{*}{ Poraneter } & \multirow{2}{*}{$\begin{array}{c}\text { Sempi } \\
\text { Depeh }\end{array}$} & \multicolumn{2}{|l|}{$\begin{array}{r}8012+6 \\
68.00\end{array}$} \\
\hline & & Result & $\theta$ \\
\hline 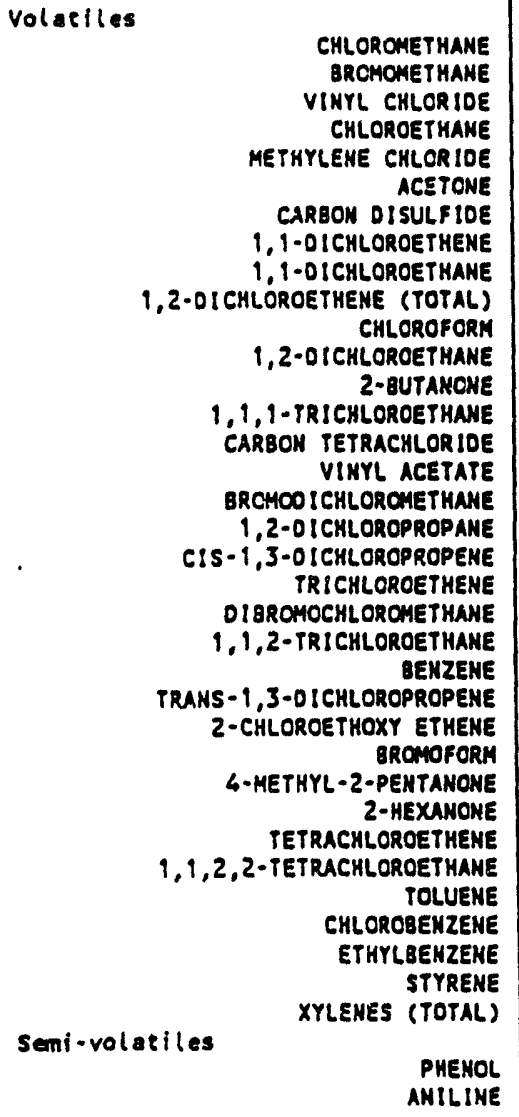 & 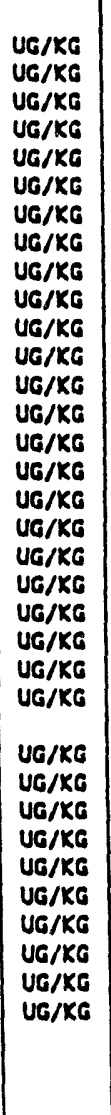 & $\begin{array}{c}11.000 \\
11.000 \\
11.000 \\
11.000 \\
5.000 \\
51.000 \\
5.000 \\
5.000 \\
5.000 \\
5.000 \\
5.000 \\
5.000 \\
11.000 \\
5.000 \\
5.000 \\
11.000 \\
5.000 \\
5.000 \\
5.000 \\
5.000 \\
5.000 \\
5.000 \\
5.000 \\
5.000 \\
11 / R \\
5.000 \\
11.000 \\
11.000 \\
5.000 \\
5.000 \\
5.000 \\
5.000 \\
5.000 \\
5.000 \\
5.000 \\
\\
11 / R \\
11 / R\end{array}$ & $\begin{array}{l}u \\
U \\
U \\
U \\
U \\
U \\
U \\
U \\
U \\
U \\
U \\
U \\
U \\
U \\
U \\
U \\
U \\
U \\
U \\
U \\
U \\
U \\
U \\
U \\
U \\
U \\
U \\
U \\
U \\
U \\
U \\
U \\
U \\
U\end{array}$ \\
\hline
\end{tabular}


WHC-SD-EN-TI-279, Rev. 0

Location 307 T-1, 399-3-15

\begin{tabular}{|c|c|c|}
\hline Poremeter & $\begin{array}{l}\text { Semply } \\
\text { Depth }\end{array}$ & $\begin{array}{r}\text { 101Rs6 } \\
68.00\end{array}$ \\
\hline & Unies & Result \\
\hline 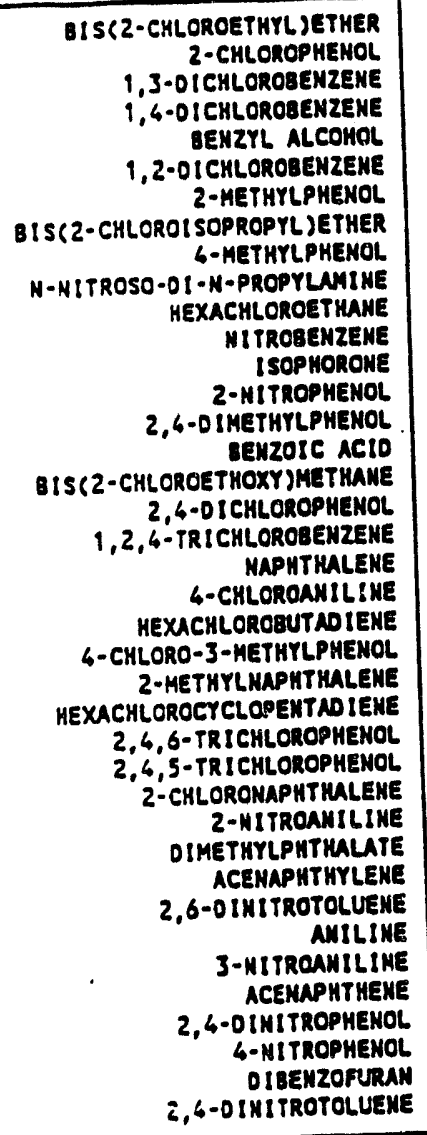 & & $\begin{array}{l}N / R \\
N / R \\
N / R \\
N / R \\
N / R \\
N / R \\
N / R \\
N / R \\
N / R \\
N / R \\
N / R \\
N / R \\
N / R \\
N / R \\
N / R \\
N / R \\
N / R \\
N / R \\
N / R \\
N / R \\
N / R \\
N / R \\
N / R \\
N / R \\
N / R \\
N / R \\
N / R \\
N / R \\
N / R \\
N / R \\
N / R \\
N / R \\
N / R \\
N / R \\
N / R \\
N / R \\
N / R \\
N / R \\
N / R \\
N / R \\
N \\
N / R \\
N\end{array}$ \\
\hline
\end{tabular}


WHC-SD-EN-TI-279, Rev. 0

Location $307+\cdot 1,399 \cdot 3 \cdot 15$

\begin{tabular}{|c|c|c|c|}
\hline \multirow[t]{2}{*}{ Parameter } & \multirow{2}{*}{$\begin{array}{l}\text { Sanow } \\
\text { Depeh }\end{array}$} & \multicolumn{2}{|l|}{$\begin{array}{r}801 R J 4 \\
60.00\end{array}$} \\
\hline & & Resule & 0 \\
\hline 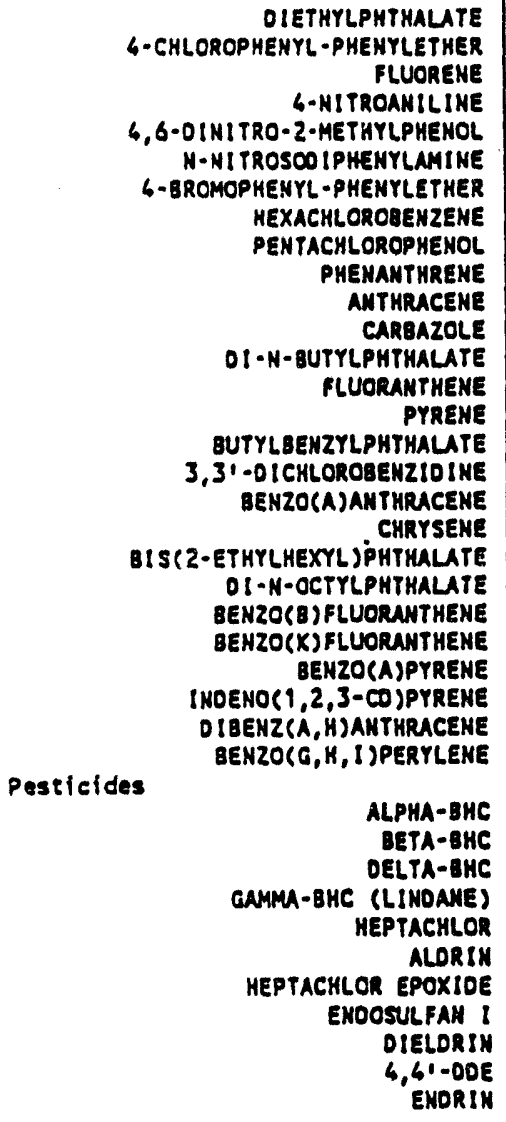 & $\begin{array}{l}\text { UG/KG } \\
\text { UG/KG } \\
\text { UG/KG } \\
\text { UG/KG } \\
\text { UG/KG } \\
\text { UG/KG } \\
\text { UG/KG } \\
\text { UG/KG } \\
\text { UG/KG } \\
\text { UG/KG } \\
\text { UG/KG }\end{array}$ & 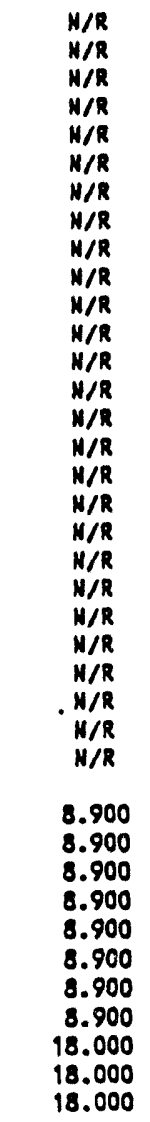 & $\begin{array}{l}u \\
u \\
u \\
u \\
u \\
u \\
u \\
u \\
u \\
u \\
u\end{array}$ \\
\hline
\end{tabular}


WHC-SD-EN-TI-279, Rev. 0

Location 307 T-1, 399-3-15

\begin{tabular}{|c|c|c|c|}
\hline \multirow[t]{2}{*}{ Porameer } & \multirow{2}{*}{$\begin{array}{l}\text { Senpl: } \\
\text { Depeh }\end{array}$} & \multicolumn{2}{|l|}{$\begin{array}{r}\text { SOIRJG } \\
68.00\end{array}$} \\
\hline & & Resule & 0 \\
\hline 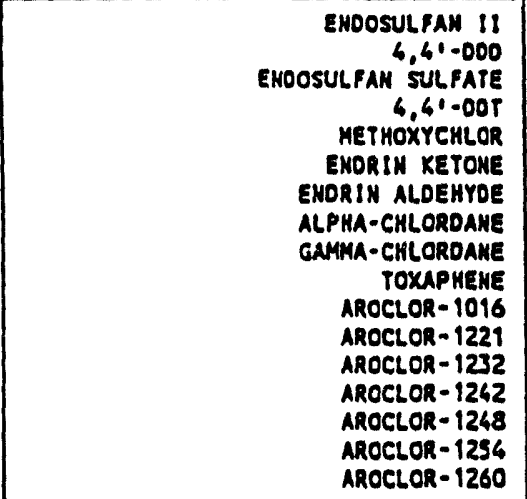 & $\begin{array}{l}\text { UG/KG } \\
\text { UG/KG } \\
\text { UG/KG } \\
\text { UG/KG } \\
\text { UG/KG } \\
\text { UG/KG } \\
\text { UG/KG } \\
\text { UG/KG } \\
\text { UG/KG } \\
\text { UG/KG } \\
\text { UG/KG } \\
\text { UG/KG } \\
\text { UG/KG } \\
\text { UG/KG } \\
\text { UG/KG } \\
\text { UG/KG }\end{array}$ & $\begin{array}{c}18.000 \\
18.000 \\
18.000 \\
18.000 \\
89.000 \\
18.000 \\
N / R \\
89.000 \\
89.000 \\
180.000 \\
89.000 \\
89.000 \\
89.000 \\
89.000 \\
89.000 \\
180.000 \\
180.000\end{array}$ & $\begin{array}{l}u \\
u \\
u \\
u \\
u \\
u \\
u \\
u \\
u \\
u \\
u \\
u \\
u \\
u \\
U \\
U\end{array}$ \\
\hline
\end{tabular}




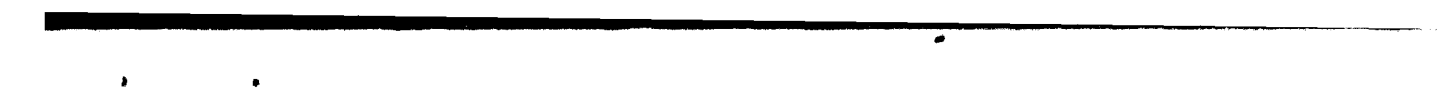

WHC-SD-EN-TI-279, Rev. 0

This page left intentionally blank. 
Locat Ion 307 I-2, 399-3-16

\begin{tabular}{|c|c|c|c|c|c|c|c|c|c|c|c|c|c|}
\hline \multirow[t]{2}{*}{ Paraneter } & \multirow{2}{*}{\begin{tabular}{|l|} 
Sampl \\
Depth \\
Units
\end{tabular}} & \multicolumn{2}{|l|}{$\begin{array}{r}8010 C 3 \\
3.00\end{array}$} & \multicolumn{2}{|l|}{$\begin{array}{c}\text { BO1CC3RE } \\
3.00\end{array}$} & \multicolumn{2}{|l|}{$\begin{array}{r}2010 C 5 \\
4.90\end{array}$} & \multicolumn{2}{|c|}{$\underset{4.90}{\text { Doicesee }}$} & \multicolumn{2}{|l|}{$\begin{array}{r}001 c c 6 \\
6.00\end{array}$} & \multicolumn{2}{|c|}{$\begin{array}{c}\text { cotccsae } \\
6.00\end{array}$} \\
\hline & & Result & 0 & Result & $a$ & Resutt & a & Result & a & Result t & a & Resull & a \\
\hline 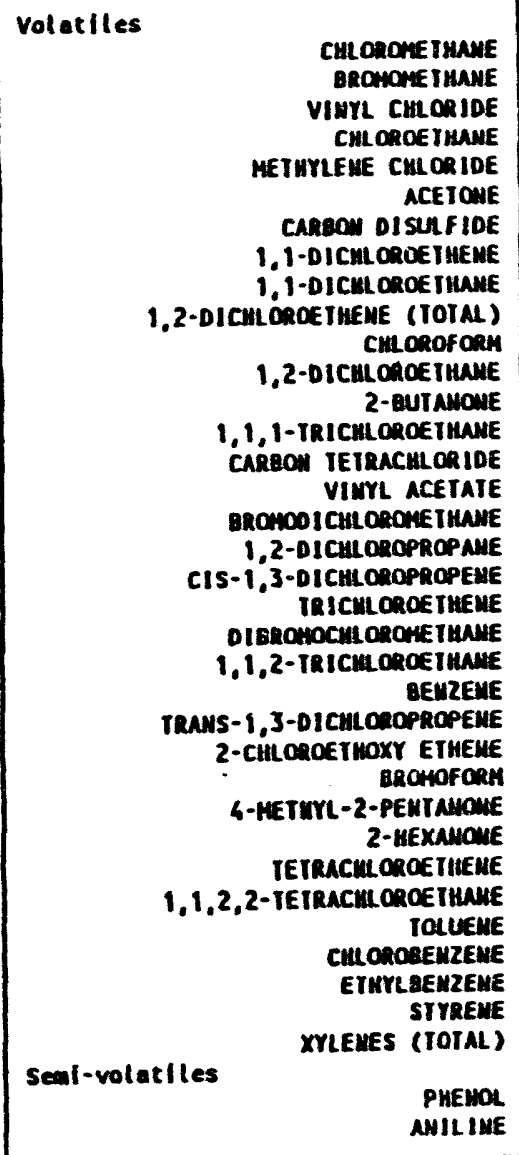 & $\begin{array}{l}U G / K G \\
U G / K G \\
U G / K G \\
U G / K G \\
U G / K G \\
U G / K G \\
U G / K G \\
U G / K G \\
U G / K G \\
U G / K G \\
U G / K G \\
U G / K G \\
U G / K G \\
U G / K G \\
U G / K G \\
U G / K G \\
U G / K G \\
U G / K G \\
U G / K G \\
U G / K G \\
U G / K G \\
U U / K G \\
U G / K G \\
U G / K G \\
U G / K G \\
U G / K G \\
U G / K G \\
U G / K G \\
U G / K G \\
U G / K G \\
U G / K G \\
U G / K G \\
U G / K G \\
U G / K G\end{array}$ & $\begin{array}{r}12.000 \\
12.000 \\
12.000 \\
12.000 \\
57.000 \\
12.000 \\
6.000 \\
6.000 \\
6.000 \\
6.000 \\
6.000 \\
6.000 \\
12.000 \\
6.000 \\
6.000 \\
12.000 \\
6.000 \\
6.000 \\
6.000 \\
160.000 \\
6.000 \\
6.000 \\
6.000 \\
6.000 \\
11 / R \\
6.000 \\
12.000 \\
12.000 \\
27.000 \\
6.000 \\
38.000 \\
6.000 \\
6.000 \\
6.000 \\
6.000 \\
11 / 2 \\
1 / 2\end{array}$ & 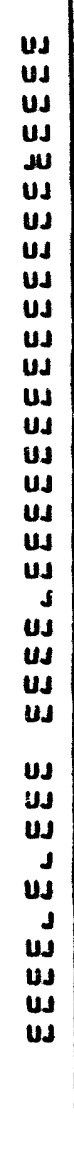 & $\begin{array}{c}12.000 \\
12.000 \\
12.000 \\
12.000 \\
61.000 \\
110.000 \\
6.000 \\
6.000 \\
6.000 \\
6.000 \\
6.000 \\
6.000 \\
12.000 \\
6.000 \\
6.000 \\
12.000 \\
6.000 \\
6.000 \\
6.000 \\
70.000 \\
6.000 \\
6.000 \\
6.000 \\
6.000 \\
11 / R \\
6.000 \\
12.000 \\
12.000 \\
6.000 \\
8.000 \\
10.000 \\
6.000 \\
6.000 \\
6.000 \\
6.000 \\
11 / R \\
11 / R \\
\end{array}$ & 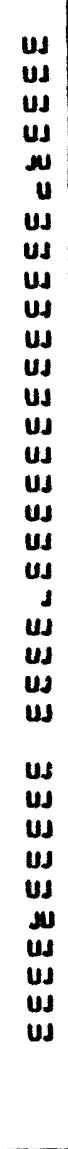 & $\begin{array}{c}11.000 \\
11.000 \\
11.000 \\
11.000 \\
15.000 \\
11.000 \\
6.000 \\
6.000 \\
6.000 \\
6.000 \\
6.000 \\
6.000 \\
11.000 \\
6.000 \\
6.000 \\
11.000 \\
6.000 \\
6.000 \\
6.000 \\
18.000 \\
6.000 \\
6.000 \\
6.000 \\
6.000 \\
11 / 2 \\
6.000 \\
11.000 \\
11.000 \\
6.000 \\
6.000 \\
8.000 \\
6.000 \\
6.000 \\
6.000 \\
6.000 \\
11 / 2 \\
11 / R\end{array}$ & 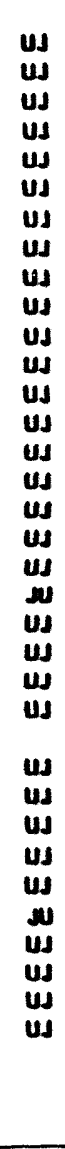 & $\begin{array}{r}11.000 \\
11.000 \\
11.000 \\
11.000 \\
26.000 \\
11.000 \\
6.000 \\
6.000 \\
6.000 \\
6.000 \\
6.000 \\
6.000 \\
11.000 \\
6.000 \\
6.000 \\
11.000 \\
6.000 \\
6.000 \\
6.000 \\
13.000 \\
6.000 \\
6.000 \\
6.000 \\
6.000 \\
1120 \\
6.000 \\
11.000 \\
11.000 \\
6.000 \\
6.000 \\
6.000 \\
6.000 \\
6.000 \\
6.000 \\
6.000 \\
\\
11 / 2 \\
11 / 2\end{array}$ & 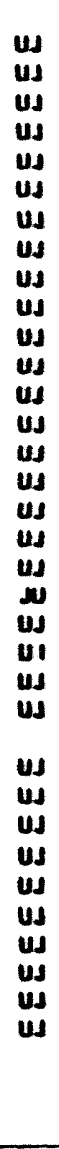 & $\begin{array}{c}10.000 \\
10.000 \\
10.000 \\
10.000 \\
14.000 \\
26.000 \\
5.000 \\
5.000 \\
5.000 \\
5.000 \\
5.000 \\
5.000 \\
10.000 \\
5.000 \\
5.000 \\
10.000 \\
5.000 \\
5.000 \\
5.000 \\
5.000 \\
5.000 \\
5.000 \\
5.000 \\
5.000 \\
1110 \\
5.000 \\
5.000 \\
10.000 \\
5.000 \\
5.000 \\
2.000 \\
5.000 \\
5.000 \\
5.000 \\
5.000 \\
\\
110 \\
11 / 1\end{array}$ & $\begin{array}{c}u s \\
u \\
u \\
u \\
u \\
u \\
u \\
u \\
u \\
u \\
u \\
u \\
u \\
u \\
u \\
u \\
u \\
u \\
u \\
u \\
u \\
u \\
u \\
u \\
u \\
u \\
u \\
u \\
u \\
u \\
u \\
u \\
u \\
u\end{array}$ & $\begin{array}{c}10.000 \\
10.000 \\
10.000 \\
10.000 \\
21.000 \\
13.000 \\
5.000 \\
5.000 \\
5.000 \\
5.000 \\
1.000 \\
5.000 \\
10.000 \\
5.000 \\
5.000 \\
10.000 \\
5.000 \\
5.000 \\
5.000 \\
5.000 \\
5.000 \\
5.000 \\
5.000 \\
5.000 \\
11 / 2 \\
5.000 \\
10.000 \\
10.000 \\
5.000 \\
5.000 \\
5.000 \\
5.000 \\
5.000 \\
5.000 \\
5.000 \\
\\
11 / 2 \\
11 / 1\end{array}$ & 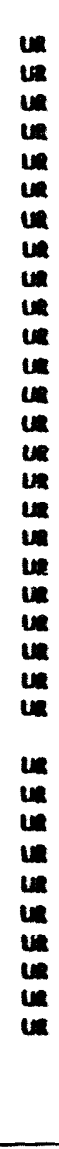 \\
\hline
\end{tabular}


WHC-SD-EN-TI-279, Rev. 0

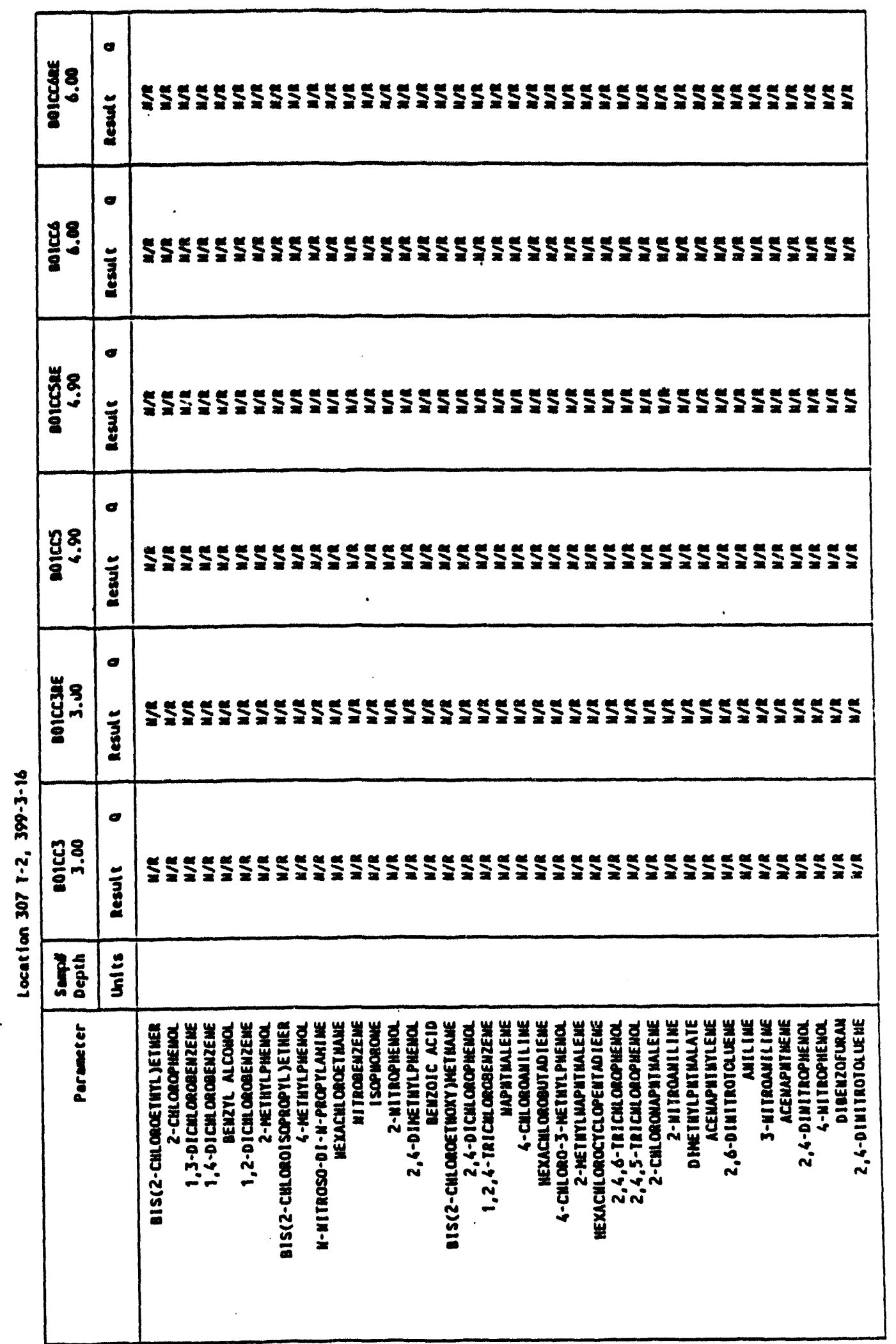


Location 307 1-2, 3x9-3-16

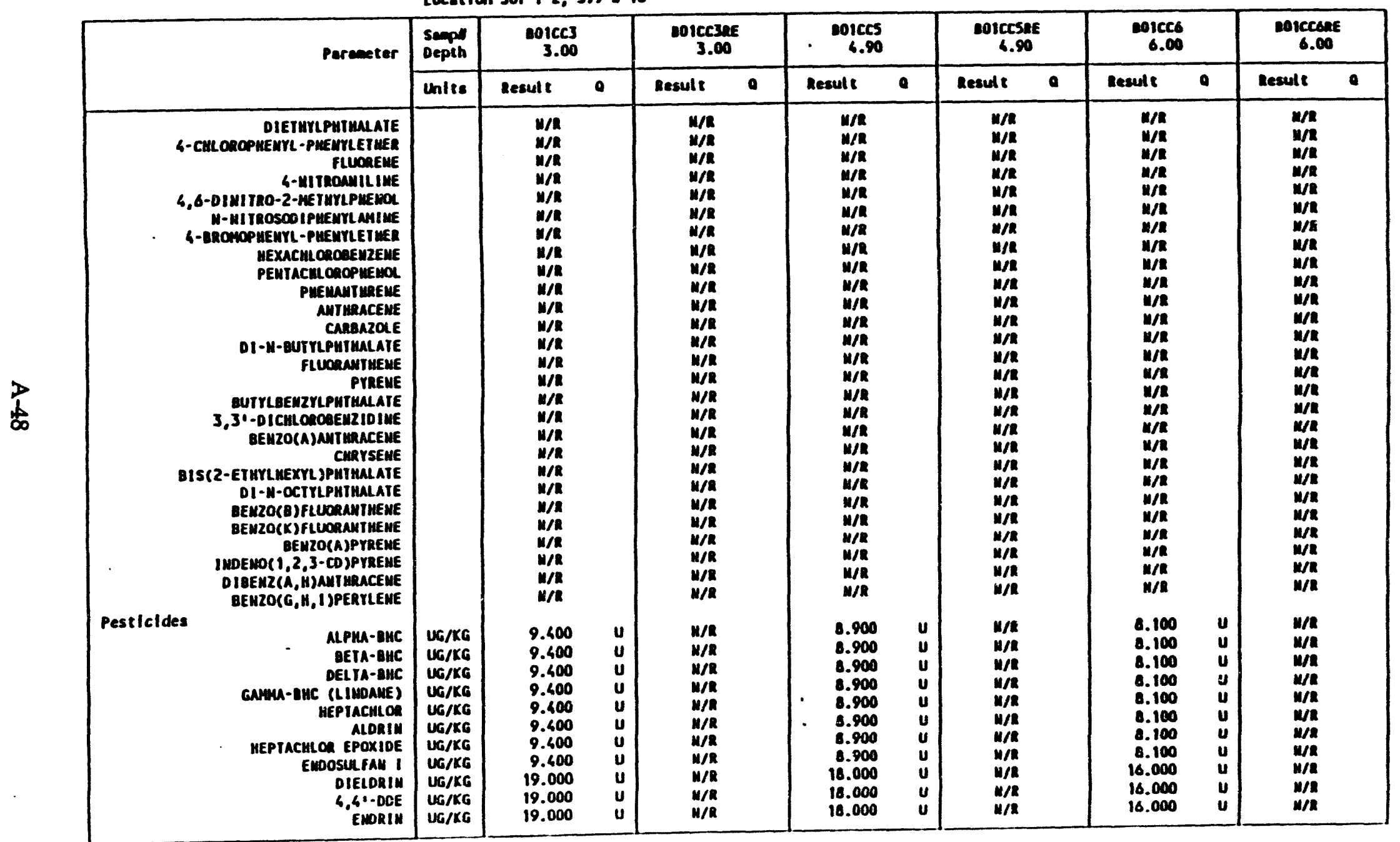




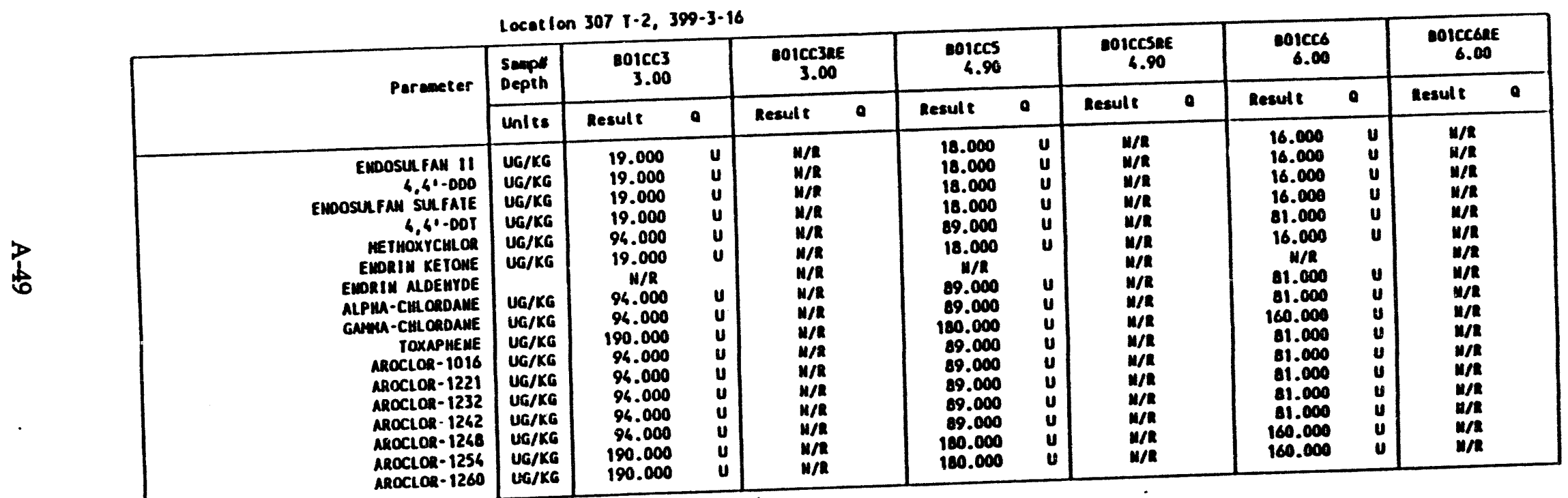




\begin{tabular}{|c|c|c|c|c|c|c|c|c|c|c|c|c|c|}
\hline \multirow[t]{2}{*}{ Parameter } & \multirow{2}{*}{$\begin{array}{l}\text { Sompt } \\
\text { Depth } \\
\text { Units }\end{array}$} & \multicolumn{2}{|l|}{$\begin{array}{r}801 c c 7 \\
10.30\end{array}$} & \multicolumn{2}{|l|}{$\begin{array}{r}801 C 04 \\
12.40\end{array}$} & \multicolumn{2}{|c|}{$\begin{array}{c}\text { BO1CDARE } \\
12.40\end{array}$} & \multicolumn{2}{|l|}{$\begin{array}{r}101 \text { COS } \\
16.30\end{array}$} & \multicolumn{2}{|c|}{$\begin{array}{c}01 \operatorname{COSRE} \\
14.30\end{array}$} & \multicolumn{2}{|l|}{$\begin{array}{r}101 C 07 \\
16.30\end{array}$} \\
\hline & & Result & a & Result & $a$ & Result & 0 & Result & 0 & Result & $a$ & Result & 0 \\
\hline 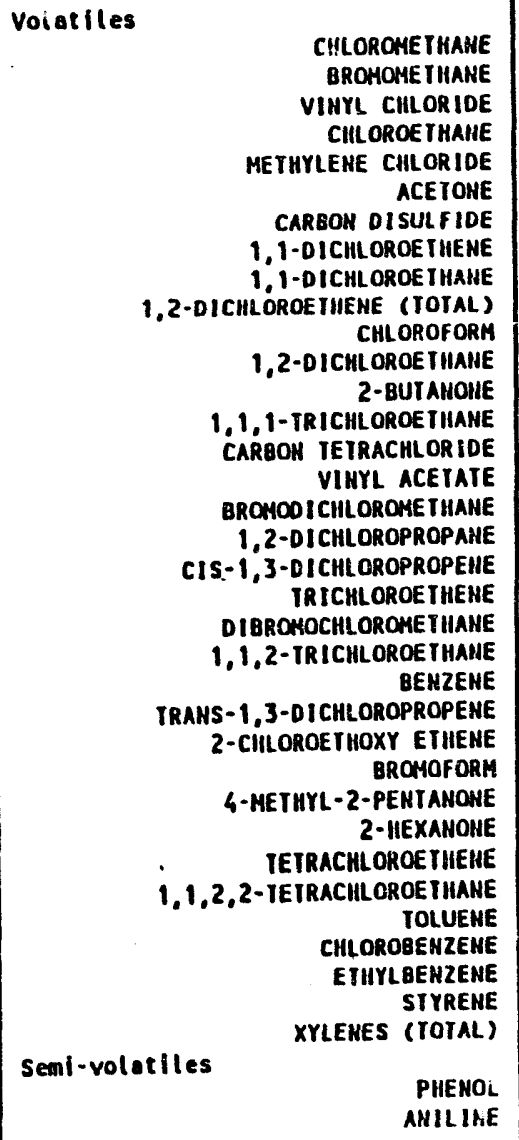 & $\begin{array}{l}U G / K G \\
U G / K G \\
U G / K G \\
U G / K G \\
U G / K G \\
U G / K G \\
U G / K G \\
U G / K G \\
U G / K G \\
U G / K G \\
U G / K G \\
U G / K G \\
U G / K G \\
U G / K G \\
U G / K G \\
U G / K G \\
U G / K G \\
U G / K G \\
U G / K G \\
U G / K G \\
U G / K G \\
U G / K G \\
U G / K G \\
U G / K G \\
U G / K G \\
U G / K G \\
U G / K G \\
U G / K G \\
U G / K G \\
U G / K G \\
U G / K G \\
U G / K G \\
U G / K G \\
U G / K G \\
U G / K G\end{array}$ & $\begin{array}{r}11.000 \\
11.000 \\
11.000 \\
11.000 \\
8.000 \\
20.000 \\
2.000 \\
5.000 \\
5.000 \\
5.000 \\
5.000 \\
5.000 \\
11.000 \\
5.000 \\
5.000 \\
11.000 \\
5.000 \\
5.000 \\
5.000 \\
6.000 \\
5.000 \\
5.000 \\
5.000 \\
5.000 \\
11 / R \\
5.000 \\
11.000 \\
11.000 \\
5.000 \\
5.000 \\
4.000 \\
5.000 \\
5.000 \\
5.000 \\
5.000 \\
\\
11 / R \\
H / R\end{array}$ & $\begin{array}{c}\mathbf{u} \\
\mathbf{u} \\
\mathbf{u} \\
\mathbf{u} \\
\mathbf{u} \\
\mathbf{u} \\
\mathbf{u} \mathbf{u} \\
\mathbf{u} \\
\mathbf{u} \\
\mathbf{u} \\
\mathbf{u} \\
\mathbf{u} \\
\mathbf{u} \\
\mathbf{u} \\
\mathbf{u} \\
\mathbf{u} \\
\mathbf{u} \\
\mathbf{u} \\
\mathbf{u} \\
\mathbf{u} \\
\mathbf{u} \\
\mathbf{u} \\
\mathbf{u} \\
\mathbf{u} \\
\mathbf{u} \\
\mathbf{u} \\
\mathbf{u} \\
\mathbf{u} \\
\mathbf{u} \\
\mathbf{u} \\
\mathbf{u} \\
\mathbf{u} \\
\mathbf{u} \\
\mathbf{u}\end{array}$ & $\begin{array}{r}11.000 \\
11.000 \\
11.000 \\
11.000 \\
8.000 \\
9.000 \\
5.000 \\
5.000 \\
5.000 \\
5.000 \\
5.000 \\
5.000 \\
11.000 \\
5.000 \\
5.000 \\
11.000 \\
5.000 \\
5.000 \\
5.000 \\
10.000 \\
5.000 \\
5.000 \\
5.000 \\
5.000 \\
1 \% / R \\
5.000 \\
11.000 \\
11.000 \\
2.000 \\
5.000 \\
9.000 \\
5.000 \\
5.000 \\
5.000 \\
5.000 \\
H / R \\
H / R \\
H / R\end{array}$ & 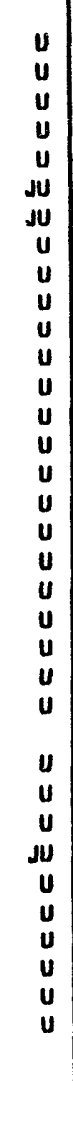 & $\begin{array}{r}11.000 \\
11.000 \\
11.000 \\
11.000 \\
27.000 \\
13.000 \\
5.000 \\
5.000 \\
5.000 \\
5.000 \\
5.000 \\
5.000 \\
11.000 \\
5.000 \\
5.000 \\
11.000 \\
5.000 \\
5.000 \\
5.000 \\
5.000 \\
5.000 \\
5.000 \\
5.000 \\
5.000 \\
11 / R \\
5.000 \\
11.000 \\
11.000 \\
5.000 \\
5.000 \\
5.000 \\
5.000 \\
5.000 \\
5.000 \\
5.000 \\
H / R \\
H / R\end{array}$ & $\begin{array}{c}\mathbf{u} \\
\mathbf{u} \\
\mathbf{u} \\
\mathbf{u} \\
\mathbf{u} \\
\text { u } \\
\mathbf{u} \\
\mathbf{u} \\
\mathbf{u} \\
\mathbf{u} \\
\mathbf{u} \\
\mathbf{u} \\
\mathbf{u} \\
\mathbf{u} \\
\mathbf{u} \\
\mathbf{u} \\
\mathbf{u} \\
\mathbf{u} \\
\mathbf{u} \\
\mathbf{u} \\
\mathbf{u} \\
\mathbf{u} \\
\mathbf{u} \\
\mathbf{u} \\
\mathbf{u} \\
\mathbf{u} \\
\mathbf{u} \\
\mathbf{u} \\
\mathbf{u} \\
\mathbf{u} \\
\mathbf{u} \\
\mathbf{u} \\
\mathbf{u}\end{array}$ & $\begin{array}{r}10.000 \\
10.000 \\
10.000 \\
10.000 \\
6.000 \\
11.000 \\
5.000 \\
5.000 \\
5.000 \\
5.000 \\
5.000 \\
5.000 \\
10.000 \\
5.000 \\
5.000 \\
10.000 \\
5.000 \\
5.000 \\
5.000 \\
4.000 \\
5.000 \\
5.000 \\
5.000 \\
5.000 \\
H / R \\
5.000 \\
10.000 \\
10.000 \\
5.000 \\
5.000 \\
8.000 \\
5.000 \\
5.000 \\
5.000 \\
5.000 \\
1 \% / R \\
11 / R\end{array}$ & $\begin{array}{c}u \\
u \\
u \\
\mathbf{u} \\
\mathbf{u} \\
\mathbf{u} \\
\mathbf{u} \\
\mathbf{u} \\
\mathbf{u} \\
\mathbf{u} \\
\mathbf{u} \\
\mathbf{u} \\
\mathbf{u} \\
\mathbf{u} \\
\mathbf{u} \\
\mathbf{u} \\
\mathbf{u} \\
\mathbf{u} \\
\mathbf{u} \\
\mathbf{u} \\
\mathbf{u} \\
\mathbf{u} \\
\mathbf{u} \\
\mathbf{u} \\
\mathbf{u} \\
\mathbf{u} \\
\mathbf{u} \\
\mathbf{u} \\
\mathbf{u} \\
\mathbf{u} \\
\mathbf{u} \\
\mathbf{u} \\
\mathbf{u} \\
\mathbf{u} \\
\mathbf{u}\end{array}$ & $\begin{array}{c}10.000 \\
10.000 \\
10.000 \\
10.000 \\
45.000 \\
31.000 \\
5.000 \\
5.000 \\
5.000 \\
5.000 \\
5.000 \\
5.000 \\
10.000 \\
5.000 \\
5.000 \\
10.000 \\
5.000 \\
5.000 \\
5.000 \\
5.000 \\
5.000 \\
5.000 \\
5.000 \\
5.000 \\
11 / R \\
5.000 \\
10.000 \\
10.000 \\
5.000 \\
5.000 \\
5.000 \\
5.000 \\
5.000 \\
5.000 \\
5.000 \\
H / R \\
H / R\end{array}$ & $\begin{array}{r}u \\
u \\
u \\
u \\
u \\
u \\
u \\
u \\
u \\
u \\
u \\
u \\
u \\
u \\
u \\
u \\
u \\
u \\
u \\
u \\
u \\
u \\
u \\
u \\
u \\
u \\
u \\
u \\
u \\
u \\
u \\
u \\
u \\
u \\
u \\
u \\
v\end{array}$ & $\begin{array}{r}10.000 \\
10.000 \\
10.000 \\
10.000 \\
5.000 \\
13.000 \\
5.000 \\
5.000 \\
5.000 \\
5.000 \\
5.000 \\
5.000 \\
10.000 \\
5.000 \\
5.000 \\
10.000 \\
5.000 \\
5.000 \\
5.000 \\
5.000 \\
5.000 \\
5.000 \\
5.000 \\
5.000 \\
H / R \\
5.000 \\
10.000 \\
10.000 \\
5.000 \\
5.000 \\
5.000 \\
5.000 \\
5.000 \\
5.000 \\
5.000 \\
H / R \\
H / R \\
\end{array}$ & $\begin{array}{l}u \\
u \\
u \\
u \\
u \\
u v \\
u \\
u \\
u \\
u \\
u \\
u \\
u \\
u \\
u \\
u \\
u \\
u \\
u \\
u \\
u \\
u \\
u \\
u \\
u \\
u \\
u \\
u \\
u \\
u \\
u \\
u \\
u \\
u \\
u \\
u\end{array}$ \\
\hline
\end{tabular}


WHC-SD-EN-TI-279, Rev. 0

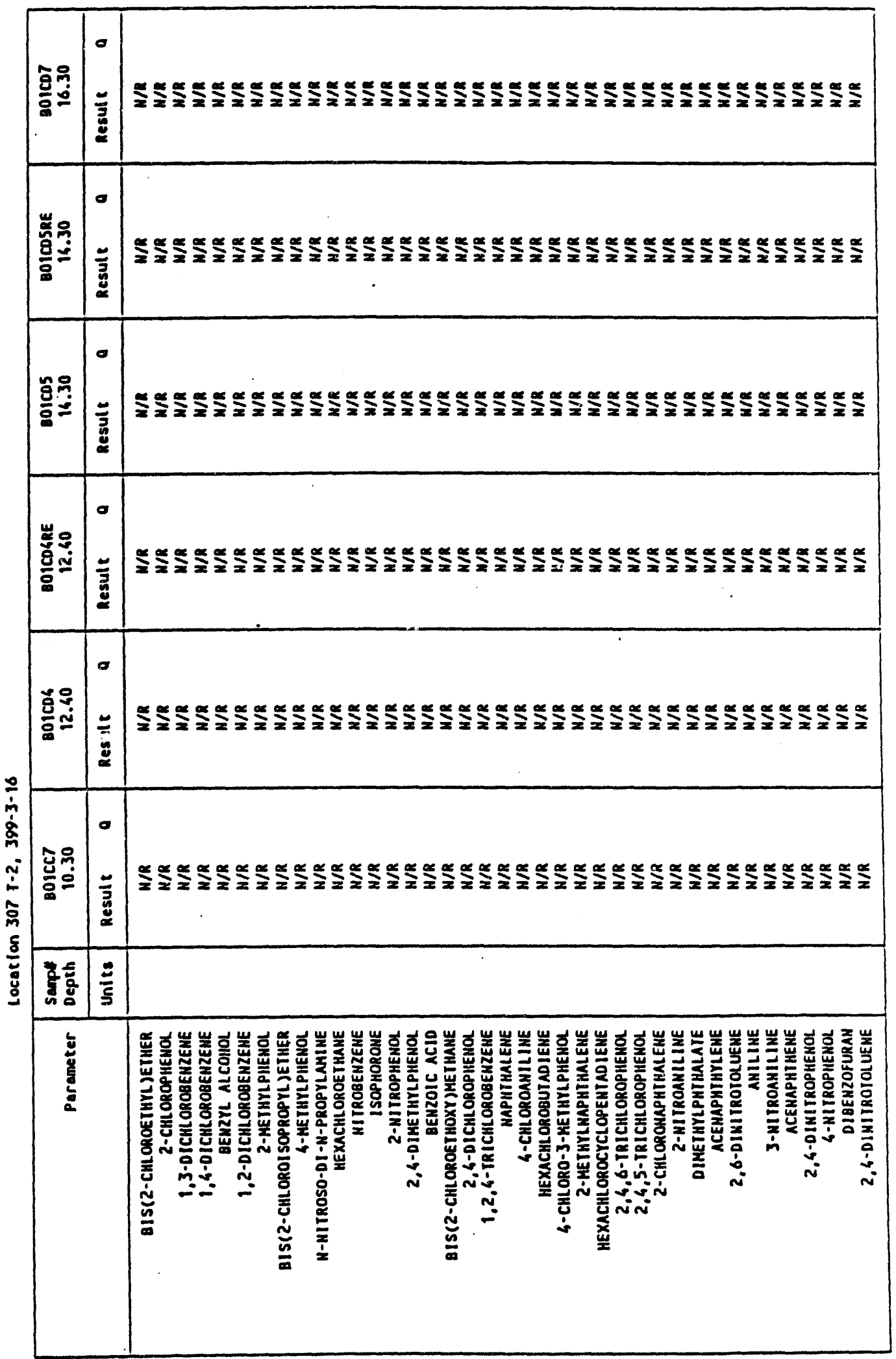


Location 307 1-2, 399-3-16

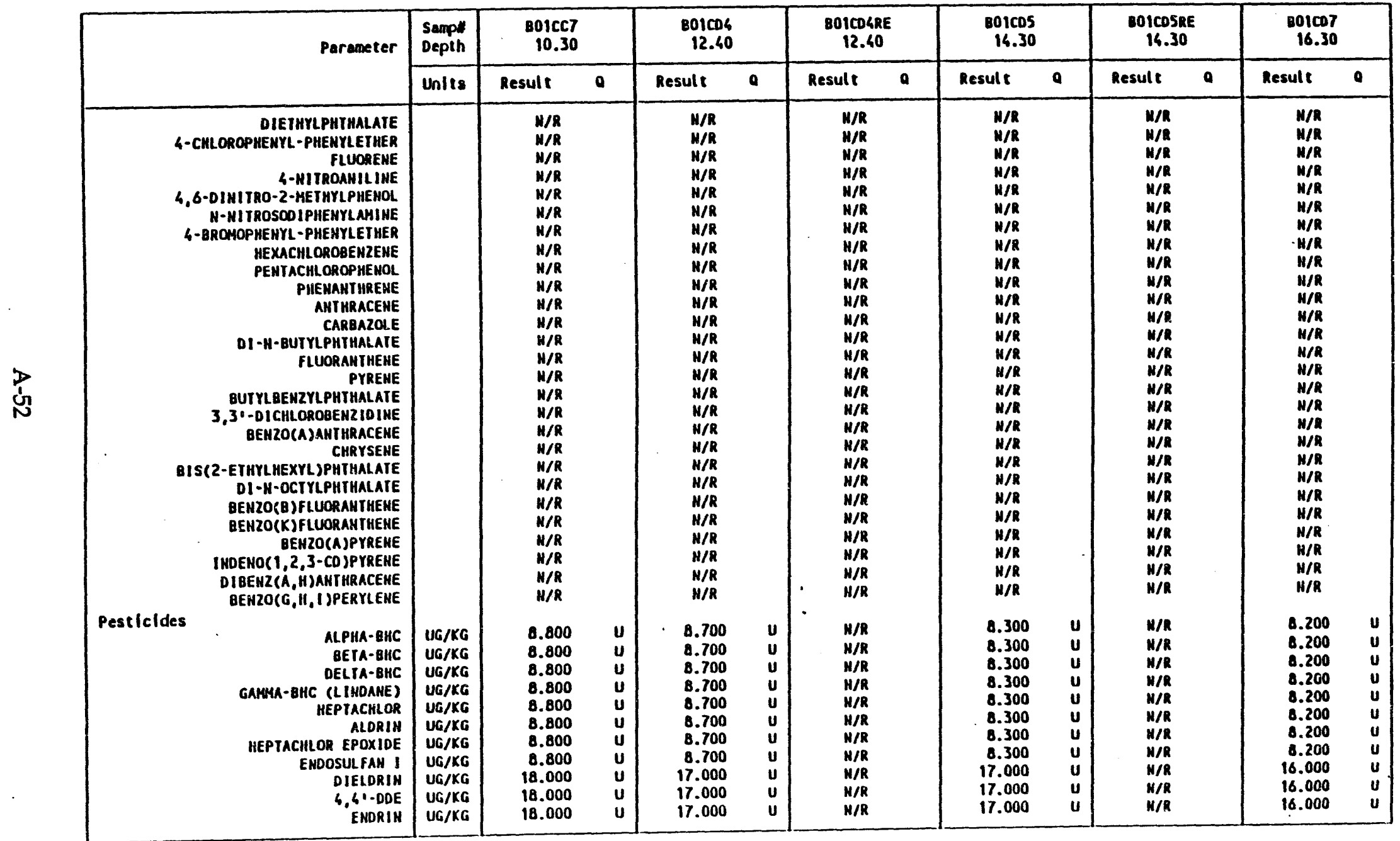




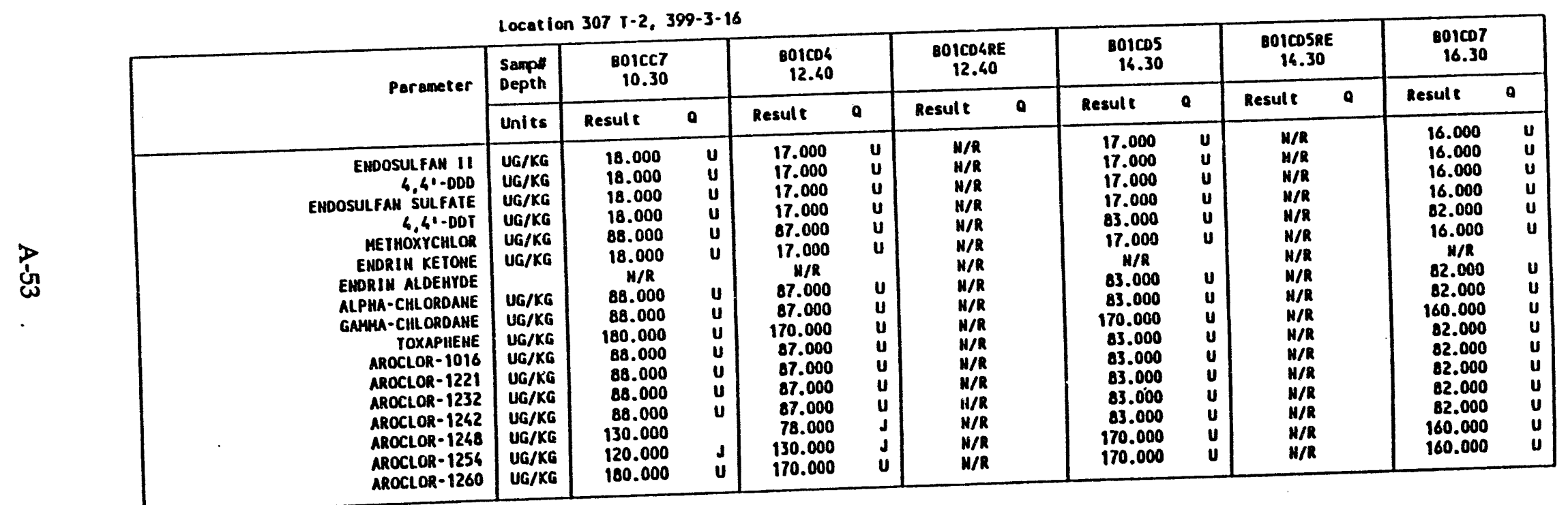


Location 307 1-2, 399-3-16

\begin{tabular}{|c|c|c|c|c|c|c|c|c|c|c|c|c|c|}
\hline \multirow[t]{2}{*}{ Parameter } & \multirow{2}{*}{$\frac{\begin{array}{l}\text { Sempk } \\
\text { Depth }\end{array}}{\text { Units }}$} & \multicolumn{2}{|l|}{$\begin{array}{r}801 C D B \\
18.40\end{array}$} & \multicolumn{2}{|l|}{$\begin{array}{r}801 C 09 \\
20.40\end{array}$} & \multicolumn{2}{|l|}{$\begin{array}{r}801 C 50 \\
22.40\end{array}$} & \multicolumn{2}{|l|}{$\begin{array}{r}\text { B01CFI } \\
24.60\end{array}$} & \multicolumn{2}{|l|}{$\begin{array}{r}\text { B01CF2 } \\
26.40\end{array}$} & \multicolumn{2}{|l|}{$\begin{array}{r}\text { B01CF3 } \\
30.70\end{array}$} \\
\hline & & Result & a & Result & 0 & Result & 0 & Result t & a & Result & a & Result & - \\
\hline 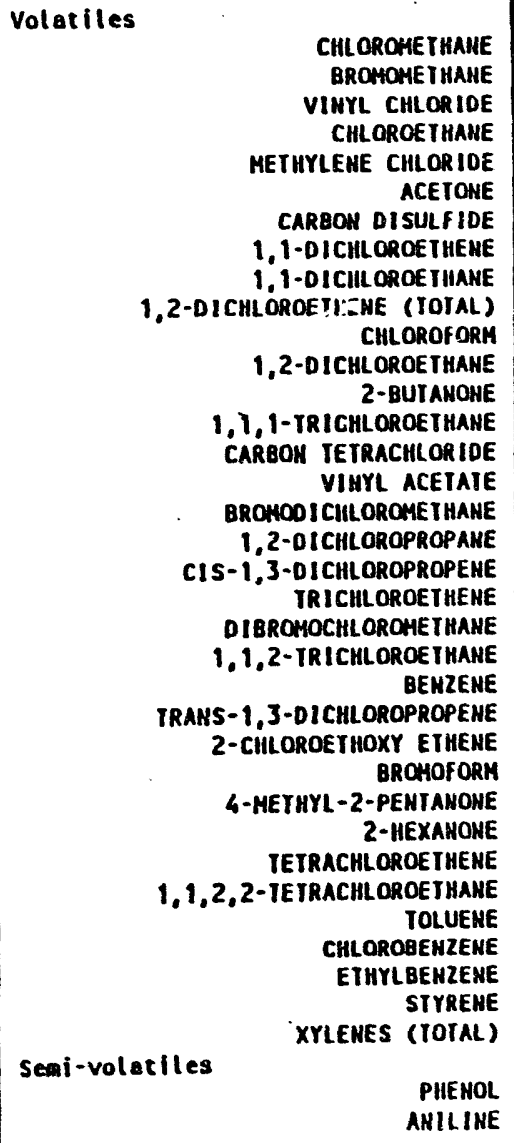 & $\begin{array}{l}U G / K G \\
U G / K G \\
U G / K G \\
U G / K G \\
U G / K G \\
U G / K G \\
U G / K G \\
U G / K G \\
U G / K G \\
U G / K G \\
U G / K G \\
U G / K G \\
U G / K G \\
U G / K G \\
U G / K G \\
U G / K G \\
U G / K G \\
U G / K G \\
U G / K G \\
U G / K G \\
U G K G \\
U G / K G \\
U G / K G \\
U G / K G \\
U G / K G \\
U G / K G \\
U G / K G \\
U G / K G \\
U G / K G \\
U G / K G \\
U G / K G \\
U G / K G \\
U G K G \\
U G / K G\end{array}$ & $\begin{array}{r}10.000 \\
10.000 \\
10.000 \\
10.000 \\
7.000 \\
16.000 \\
5.000 \\
5.000 \\
5.000 \\
5.000 \\
5.000 \\
5.000 \\
10.000 \\
5.000 \\
5.000 \\
10.000 \\
5.000 \\
5.000 \\
5.000 \\
5.000 \\
5.000 \\
5.000 \\
5.000 \\
5.000 \\
H / R \\
5.000 \\
10.000 \\
10.000 \\
5.000 \\
5.000 \\
3.000 \\
5.000 \\
5.000 \\
5.000 \\
5.000 \\
\\
H / R \\
H / R\end{array}$ & $\begin{array}{c}u \\
u \\
u \\
u \\
u \\
u \\
u \\
u \\
u \\
u \\
u \\
u \\
u \\
u \\
u \\
u \\
u \\
u \\
u \\
u \\
u \\
u \\
u \\
u \\
u \\
u \\
u \\
u \\
u \\
u \\
u \\
u \\
u \\
u\end{array}$ & $\begin{array}{r}10.000 \\
10.000 \\
10.000 \\
10.000 \\
8.000 \\
20.000 \\
3.000 \\
5.000 \\
5.000 \\
5.000 \\
5.000 \\
5.000 \\
10.000 \\
5.000 \\
5.000 \\
10.000 \\
5.000 \\
5.000 \\
5.000 \\
2.000 \\
5.000 \\
5.000 \\
5.000 \\
5.000 \\
H / R \\
5.000 \\
4.000 \\
10.000 \\
5.000 \\
5.000 \\
4.000 \\
5.000 \\
5.000 \\
5.000 \\
5.000 \\
\\
H / R \\
H / R\end{array}$ & $\begin{array}{c}u \\
u \\
u \\
u \\
u \\
u \\
\mathbf{u} \\
\mathbf{u} \\
\mathbf{u} \\
\mathbf{u} \\
\mathbf{u} \\
\mathbf{u} \\
\mathbf{u} \\
\mathbf{u} \\
\mathbf{u} \\
\mathbf{u} \\
\mathbf{u} \\
\mathbf{u} \\
\mathbf{u} \\
\mathbf{u} \\
\mathbf{u} \\
\mathbf{u} \\
\mathbf{u} \\
\mathbf{u} \\
\mathbf{u} \\
\mathbf{J} \\
\mathbf{u} \\
\mathbf{u} \\
\mathbf{u} \\
\mathbf{J} \\
\mathbf{u} \\
\mathbf{u} \\
\mathbf{u} \\
\mathbf{u}\end{array}$ & $\begin{array}{r}10.000 \\
10.000 \\
10.000 \\
10.000 \\
6.000 \\
21.000 \\
5.000 \\
5.000 \\
5.000 \\
5.000 \\
5.000 \\
5.000 \\
10.000 \\
5.000 \\
5.000 \\
10.000 \\
5.000 \\
5.000 \\
5.000 \\
1.000 \\
5.000 \\
5.000 \\
5.000 \\
5.000 \\
H / R \\
5.000 \\
10.000 \\
10.000 \\
5.000 \\
5.000 \\
2.000 \\
5.000 \\
5.000 \\
5.000 \\
5.000 \\
H / R \\
H / R\end{array}$ & $\begin{array}{c}u \\
u \\
u \\
u \\
u \\
u \\
u \\
u \\
u \\
u \\
u \\
u \\
u \\
u \\
u \\
u \\
u \\
u \\
u \\
j u \\
u \\
u \\
u \\
u \\
u \\
u \\
u \\
u \\
u \\
u \\
j u \\
u \\
u \\
u \\
u\end{array}$ & $\begin{array}{r}10.000 \\
10.000 \\
10.000 \\
10.000 \\
8.000 \\
13.000 \\
5.000 \\
5.000 \\
5.000 \\
5.000 \\
5.000 \\
5.000 \\
10.000 \\
5.000 \\
5.000 \\
10.000 \\
5.000 \\
5.000 \\
5.000 \\
5.000 \\
5.000 \\
5.000 \\
5.000 \\
5.000 \\
H / R \\
5.000 \\
10.000 \\
10.000 \\
5.000 \\
5.000 \\
5.000 \\
5.000 \\
5.000 \\
5.000 \\
5.000 \\
H / R \\
H / R\end{array}$ & $\begin{array}{l}u \\
u \\
u \\
u \\
u \\
u \\
u \\
u \\
u \\
u \\
u \\
u \\
u \\
u \\
u \\
u \\
u \\
u \\
u \\
u \\
u \\
u \\
u \\
u \\
u \\
u \\
u \\
u \\
u \\
u \\
u \\
u \\
u \\
u \\
u\end{array}$ & $\begin{array}{c}10.000 \\
10.000 \\
10.000 \\
10.000 \\
11.000 \\
32.000 \\
2.000 \\
5.000 \\
5.000 \\
5.000 \\
5.000 \\
5.000 \\
10.000 \\
5.000 \\
5.000 \\
10.000 \\
5.000 \\
5.000 \\
5.000 \\
5.000 \\
5.000 \\
5.000 \\
55000 \\
5.000 \\
1 / R \\
5.000 \\
10.000 \\
10.000 \\
5.000 \\
5.000 \\
1.000 \\
5.000 \\
5.000 \\
5.000 \\
5.000 \\
14 / R \\
14 / R\end{array}$ & $\begin{array}{c}u \\
u \\
u \\
u \\
u \\
u \\
u \\
u \\
u \\
u \\
u \\
u \\
u \\
u \\
u \\
u \\
u \\
u \\
u \\
u \\
u \\
u \\
u \\
u \\
u \\
u \\
u \\
u \\
u \\
u \\
u \\
u \\
u \\
u \\
u\end{array}$ & $\begin{array}{r}10.000 \\
10.000 \\
10.000 \\
10.000 \\
14.000 \\
36.000 \\
3.000 \\
5.000 \\
5.000 \\
5.000 \\
0.900 \\
5.000 \\
10.000 \\
5.000 \\
5.000 \\
10.000 \\
5.000 \\
5.000 \\
5.000 \\
5.000 \\
5.000 \\
5.000 \\
5.000 \\
5.000 \\
H / R \\
5.000 \\
3.000 \\
10.000 \\
5.000 \\
5.000 \\
1.000 \\
5.000 \\
5.000 \\
5.000 \\
5.000 \\
\\
H / R \\
H / R\end{array}$ & $\begin{array}{c}u \\
u \\
u \\
u \\
u \\
u \\
u u \\
u \\
u \\
u \\
u u \\
u \\
u \\
u \\
u \\
u \\
u \\
u \\
u \\
u \\
u \\
u \\
u \\
u \\
u \\
u \\
u \\
u \\
u \\
u \\
\mathbf{u} \\
\mathbf{u} \\
\mathbf{u} \\
\mathbf{u} \\
\mathbf{u}\end{array}$ \\
\hline
\end{tabular}


WHC-SD-EN-TI-279, Rev. 0

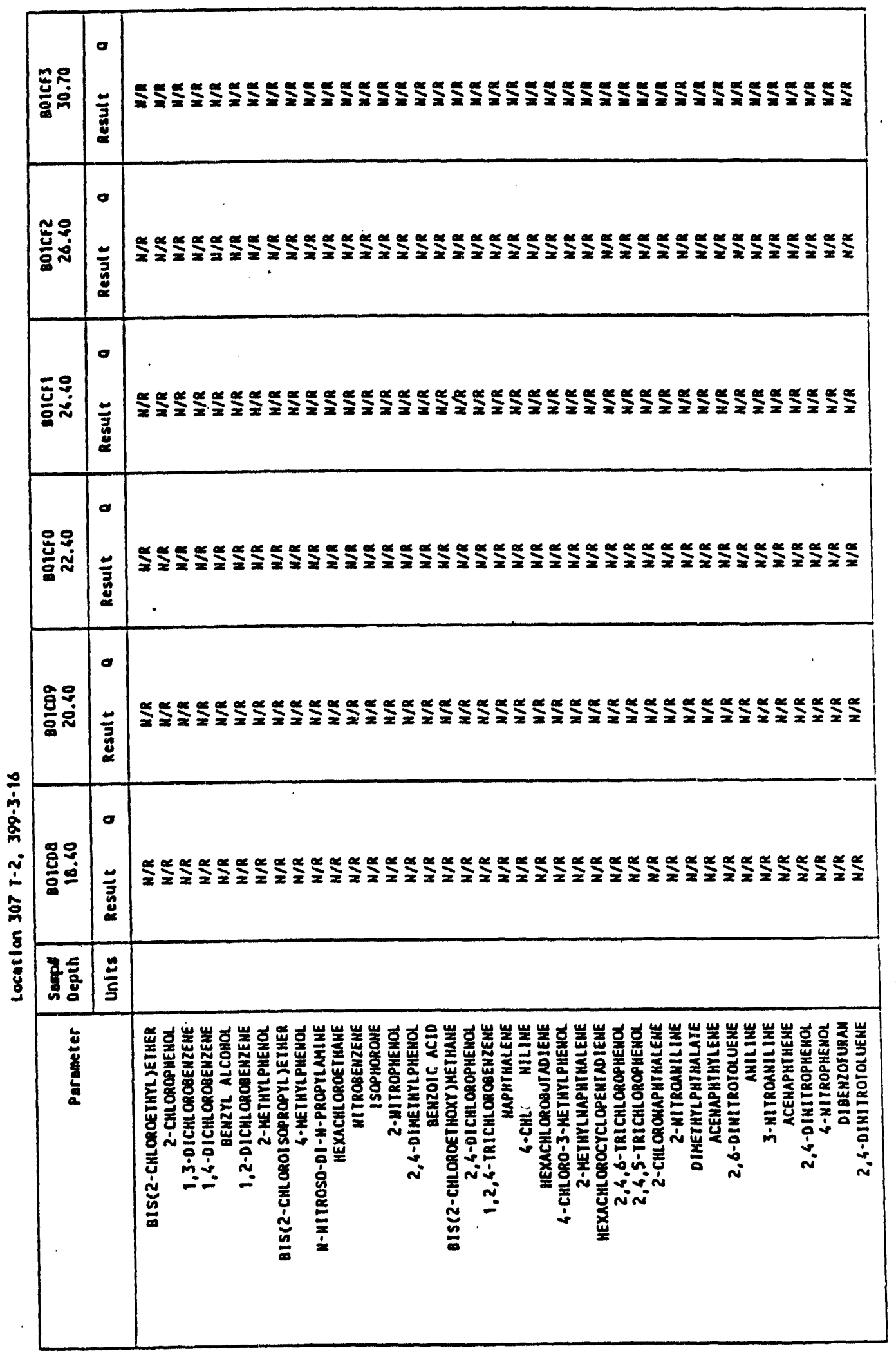


Location 307 r-2, 399-3-16

\begin{tabular}{|c|c|c|c|c|c|c|c|c|c|c|c|c|c|}
\hline \multirow[t]{2}{*}{ Parometer } & \multirow{2}{*}{\begin{tabular}{|c|}
$\begin{array}{c}\text { Sesp: } \\
\text { Depth }\end{array}$ \\
Units
\end{tabular}} & \multicolumn{2}{|l|}{$\begin{array}{r}801 C 08 \\
18.40\end{array}$} & \multicolumn{2}{|l|}{$\begin{array}{r}801 C 09 \\
20.60\end{array}$} & \multicolumn{2}{|l|}{$\begin{array}{r}\text { B01CFO } \\
22.40\end{array}$} & \multicolumn{2}{|l|}{$\begin{array}{r}\text { BOICF1 } \\
24.40\end{array}$} & \multicolumn{2}{|l|}{$\begin{array}{r}801 \text { CF2 } \\
26.40\end{array}$} & \multicolumn{2}{|l|}{$\begin{array}{r}201 c F 3 \\
30.70\end{array}$} \\
\hline & & Result & & Result & $\mathbf{a}$ & Result & & Result & 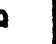 & Result & a & Result & a \\
\hline 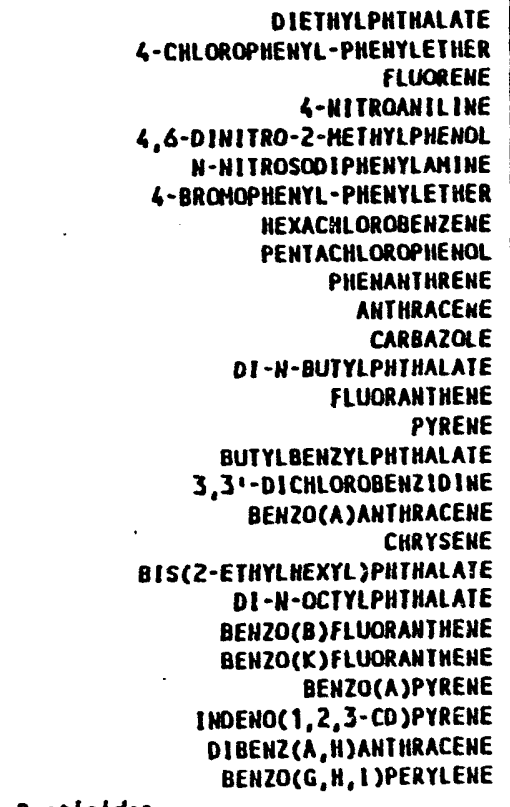 & & 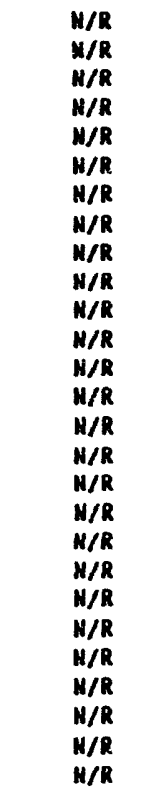 & & 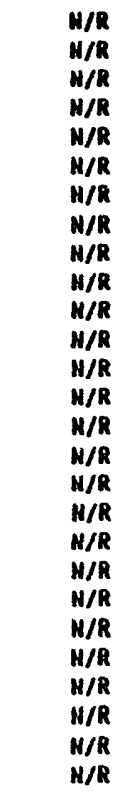 & • & 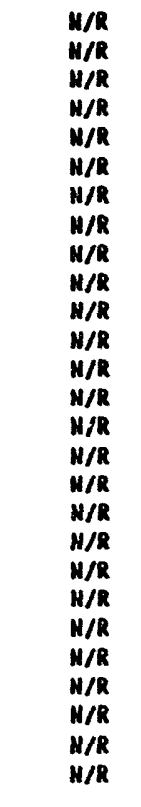 & & 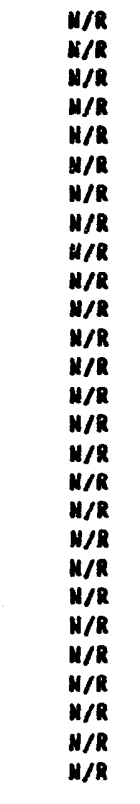 & & $\begin{array}{l}H / R \\
H / R \\
H / R \\
H / R \\
H / R \\
H / R \\
H / R \\
H / R \\
H / R \\
H / R \\
H / R \\
H / R \\
H / R \\
H / R \\
H / R \\
H / R \\
H / R \\
H / R \\
H / R \\
H / R \\
H / R \\
H / R \\
H / R \\
H / R \\
H / R \\
H / R \\
H / R \\
H / R\end{array}$ & & $\begin{array}{l}H / R \\
H / R \\
H / R \\
H / R \\
H / R \\
H / R \\
H / R \\
H / R \\
H / R \\
H / R \\
H / R \\
H / R \\
H / R \\
H / R \\
H / R \\
H / R \\
H / R \\
H / R \\
H / R \\
H / R \\
H / R \\
H / R \\
H / R \\
H / R \\
H / R \\
H / R \\
H / R \\
H / R \\
K / R \\
W / R\end{array}$ & \\
\hline 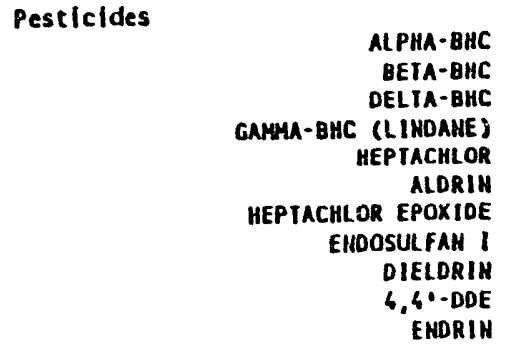 & $\begin{array}{l}U G / K G \\
U G / K G \\
U G / K G \\
U G / K G \\
U G / K G \\
U G / K G \\
U G / K G \\
U G / K G \\
U G / K G \\
U G / K G \\
U G / K G\end{array}$ & $\begin{array}{r}8.300 \\
8.300 \\
8.300 \\
8.300 \\
8.300 \\
8.300 \\
8.300 \\
8.300 \\
17.000 \\
17.000 \\
17.000\end{array}$ & $\begin{array}{l}\mathbf{u} \\
\mathbf{u} \\
\mathbf{u} \\
\mathbf{u} \\
\mathbf{u} \\
\mathbf{u} \\
\mathbf{u} \\
\mathbf{u} \\
\mathbf{u} \\
\mathbf{u} \\
\mathbf{u}\end{array}$ & $\begin{array}{r}0.300 \\
8.300 \\
8.300 \\
8.300 \\
8.300 \\
8.300 \\
8.300 \\
8.300 \\
17.000 \\
17.000 \\
17.000\end{array}$ & $\begin{array}{l}u \\
u \\
u \\
u \\
u \\
u \\
u \\
u \\
u \\
u \\
u \\
u\end{array}$ & $\begin{array}{r}8.200 \\
8.200 \\
8.200 \\
8.200 \\
8.200 \\
8.200 \\
8.200 \\
8.200 \\
16.000 \\
16.000 \\
16.000\end{array}$ & $\begin{array}{l}u \\
u \\
u \\
u \\
u \\
u \\
u \\
u \\
u \\
u \\
u\end{array}$ & $\begin{array}{r}8.300 \\
8.300 \\
8.300 \\
8.300 \\
8.300 \\
8.300 \\
8.300 \\
8.300 \\
17.000 \\
17.000 \\
17.000\end{array}$ & $\begin{array}{l}u \\
u \\
u \\
u \\
u \\
u \\
u \\
u \\
u \\
u \\
u\end{array}$ & $\begin{array}{r}8.100 \\
8.100 \\
8.100 \\
8.100 \\
8.100 \\
8.100 \\
8.100 \\
8.100 \\
16.000 \\
16.000 \\
16.000\end{array}$ & $\begin{array}{l}u \\
u \\
u \\
u \\
u \\
u \\
u \\
u \\
u \\
u \\
u\end{array}$ & $\begin{array}{r}8.300 \\
8.300 \\
8.300 \\
8.300 \\
8.300 \\
8.300 \\
8.300 \\
8.300 \\
17.000 \\
17.000 \\
17.000\end{array}$ & $\begin{array}{l}u \\
u \\
u \\
u \\
u \\
u \\
u \\
u \\
u \\
u \\
u\end{array}$ \\
\hline
\end{tabular}




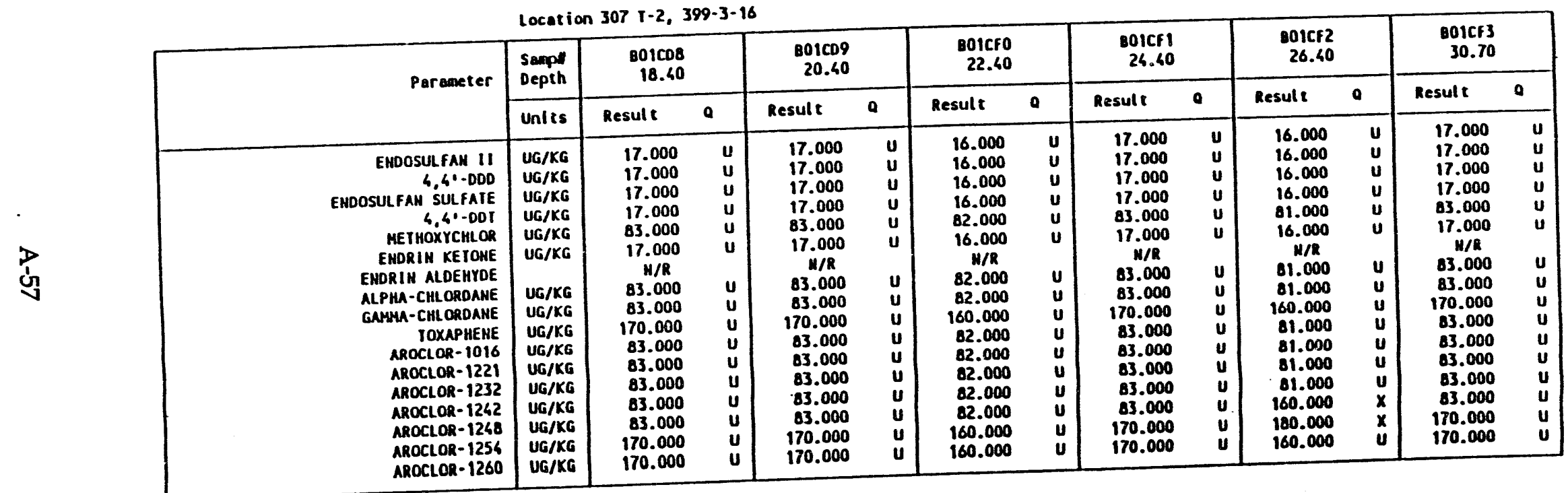


Location 307 Y-2, 399-3-16

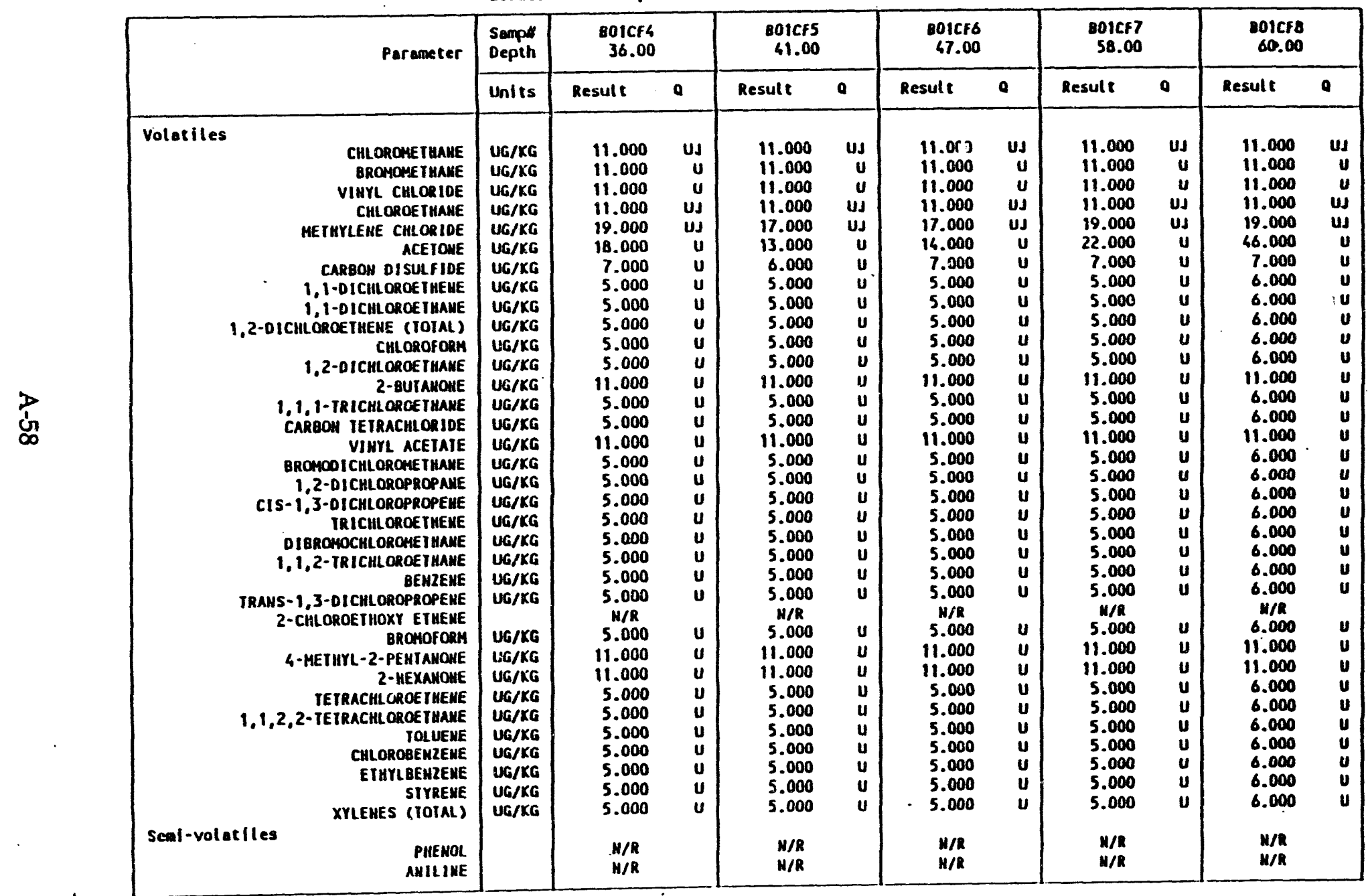


Location 307 I-2, 399-3-16

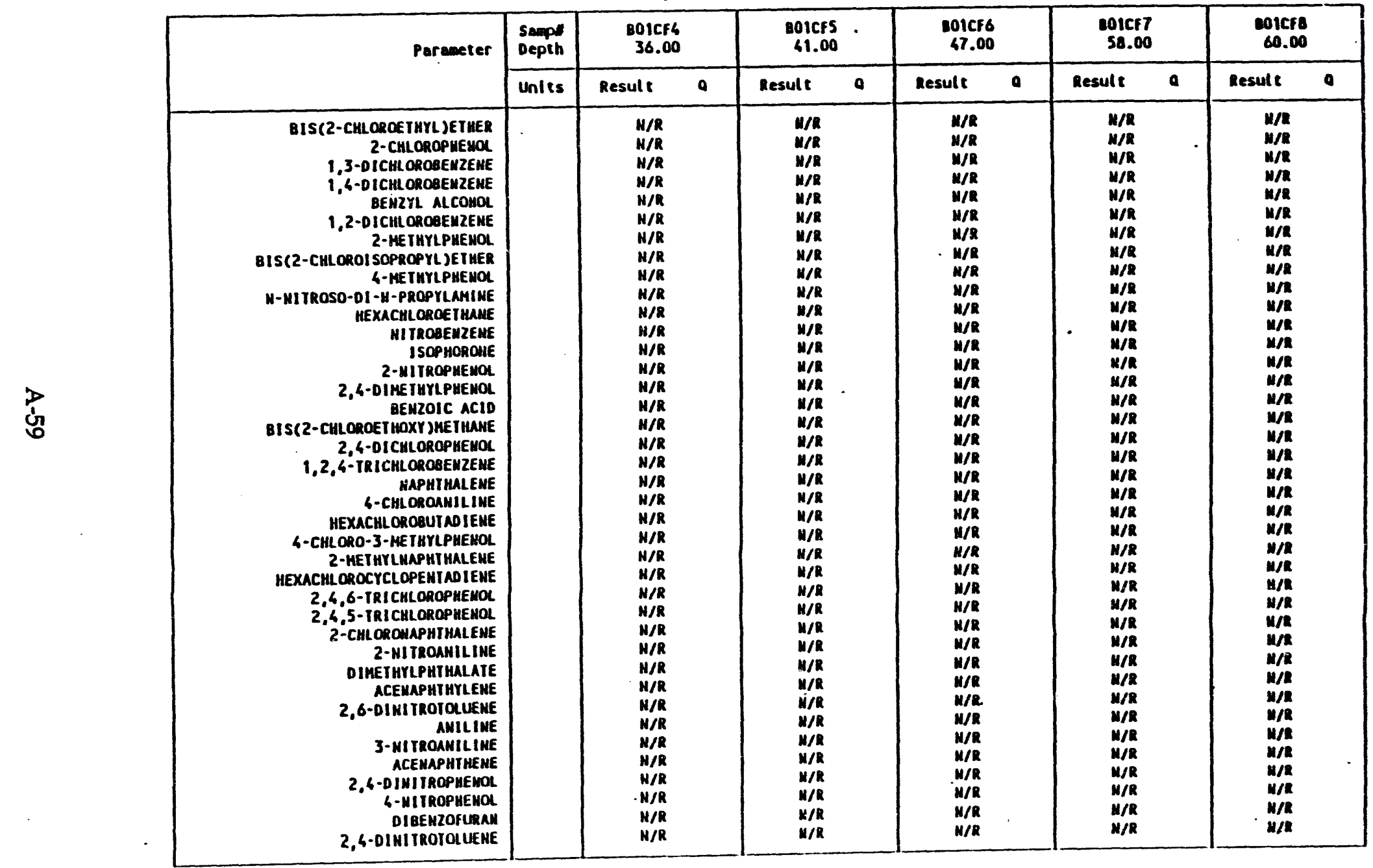


Location 307 T-2, 399-3-16

\begin{tabular}{|c|c|c|c|c|c|c|c|c|c|c|c|}
\hline \multirow[t]{2}{*}{ Parameter } & \multirow{2}{*}{\begin{tabular}{|l|}
$\begin{array}{l}\text { Sespl } \\
\text { Depth }\end{array}$ \\
Units \\
\end{tabular}} & \multicolumn{2}{|l|}{$\begin{array}{r}\text { Bo1CF4 } \\
36.00\end{array}$} & \multicolumn{2}{|l|}{$\begin{array}{r}201 C F 5 \\
41.00\end{array}$} & \multicolumn{2}{|l|}{$\begin{array}{r}001 \mathrm{CF} 6 \\
47.00\end{array}$} & \multicolumn{2}{|l|}{$\begin{array}{r}\text { Boicf7 } \\
58.00\end{array}$} & \multicolumn{2}{|l|}{$\begin{array}{r}\text { Dolcro } \\
60.09\end{array}$} \\
\hline & & Result & a & Result & $a$ & Result & 0 & Result & $a$ & Result & a \\
\hline
\end{tabular}


Location 307 1-2, 399-3-16

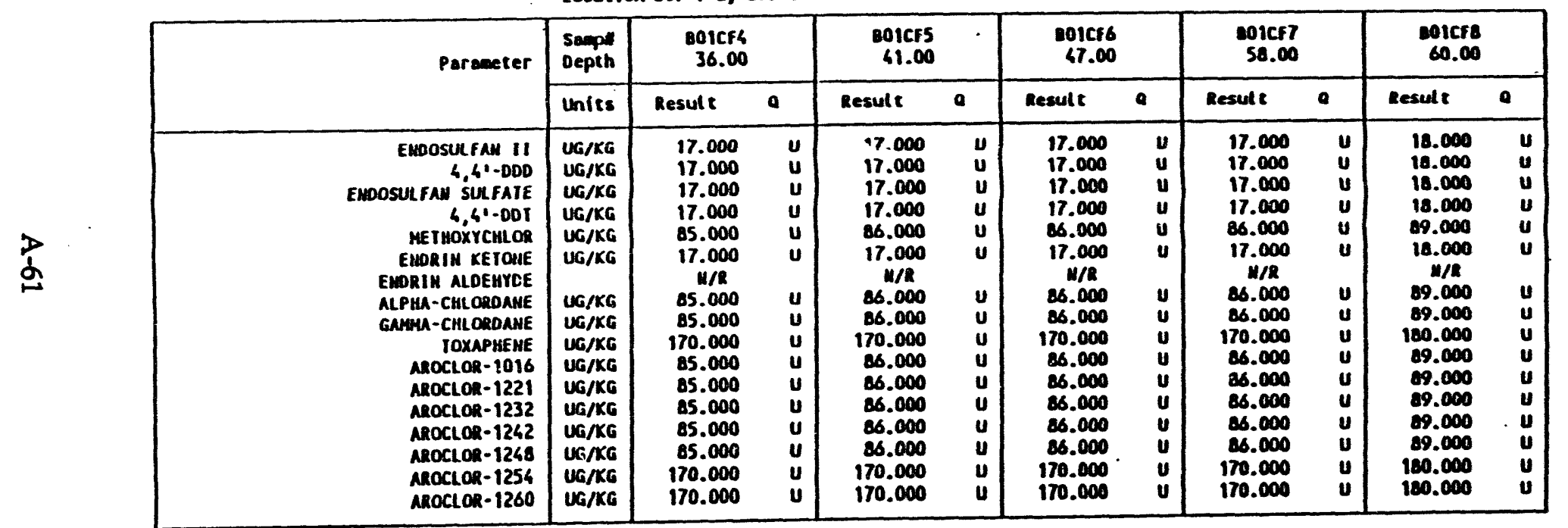


WHC-SD-EN-TI-279, Rev. 0

This page left intentionally blank. 
Location 307 r-3, 399-3-17

\begin{tabular}{|c|c|c|c|c|c|c|c|c|c|c|c|c|c|}
\hline \multirow[t]{2}{*}{ Paraneter } & \multirow{2}{*}{$\begin{array}{l}\text { Semp: } \\
\text { Depth }\end{array}$} & \multicolumn{2}{|l|}{$\begin{array}{r}\text { R01R.J5 } \\
8.40\end{array}$} & \multicolumn{2}{|l|}{$\begin{array}{r}\text { Bo1RJ7 } \\
10.40\end{array}$} & \multicolumn{2}{|l|}{$\begin{array}{r}00112.49 \\
13.60\end{array}$} & \multicolumn{2}{|l|}{$\begin{array}{l}\text { Dolexko } \\
15.50\end{array}$} & \multicolumn{2}{|l|}{$\begin{array}{l}\text { solaxi } \\
17.70\end{array}$} & \multicolumn{2}{|l|}{$\begin{array}{r}\text { miax } 2 \\
20.00\end{array}$} \\
\hline & & Result & a & Result & a & Result & 0 & Result & 0 & Result & 0 & Result t & a \\
\hline 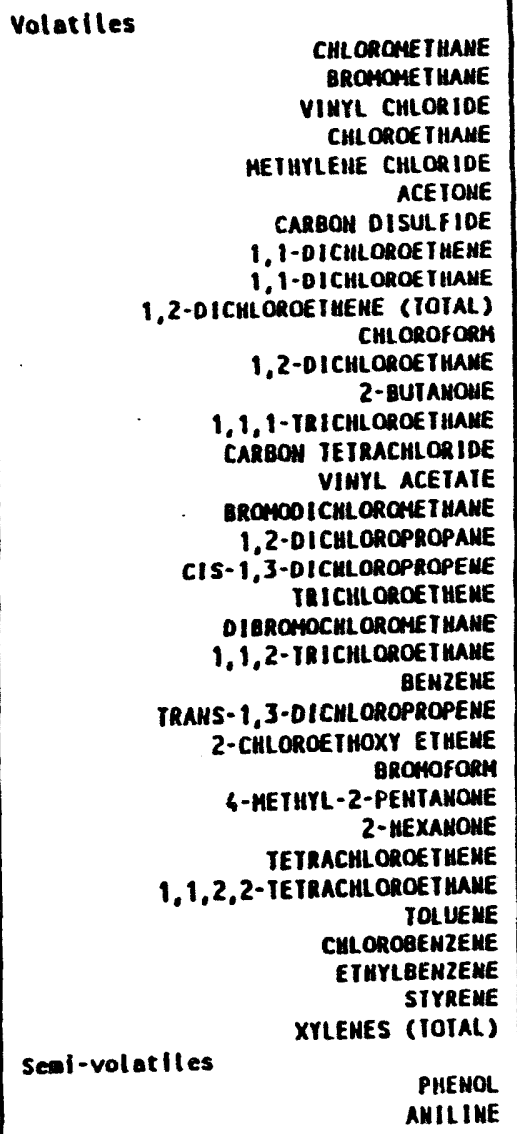 & $\begin{array}{l}U G / K G \\
U G / K G \\
U G / K G \\
U G / K G \\
U G / K G \\
U G / K G \\
U G / K G \\
U G / K G \\
U G / K G \\
U G / K G \\
U G / K G \\
U G / K G \\
U G / K G \\
U G / K G \\
U G / K G \\
U G / K G \\
U G / K G \\
U G / K G \\
U G / K G \\
U G / K G \\
U G / K G \\
U G / K G \\
U G / K G \\
U G / K G \\
U G / K G \\
U G / K G \\
U G / K G \\
U G / K G \\
U G / K G \\
U G / K G \\
U G / K G \\
U G / K G \\
U G K G \\
U G / K G\end{array}$ & $\begin{array}{r}11.000 \\
11.000 \\
11.000 \\
11.000 \\
16.000 \\
11.000 \\
6.000 \\
6.000 \\
6.000 \\
6.000 \\
6.000 \\
6.000 \\
11.000 \\
6.000 \\
6.000 \\
11.000 \\
6.000 \\
6.000 \\
6.000 \\
6.000 \\
6.000 \\
6.000 \\
6.000 \\
6.000 \\
11 / R \\
6.000 \\
11.000 \\
11.000 \\
6.000 \\
6.000 \\
6.000 \\
6.000 \\
6.000 \\
6.000 \\
6.000 \\
1 W / R \\
W / R\end{array}$ & $\begin{array}{l}u \\
u \\
u \\
u \\
u \\
u \\
u \\
u \\
u \\
u \\
u \\
u \\
u \\
u \\
u \\
u \\
u \\
u \\
u \\
u \\
u \\
u \\
u \\
u \\
u \\
u \\
u \\
u \\
u \\
u \\
u \\
u \\
u \\
u \\
u\end{array}$ & $\begin{array}{r}11.000 \\
11.000 \\
11.000 \\
11.000 \\
12.000 \\
11.000 \\
5.000 \\
5.000 \\
5.000 \\
5.000 \\
5.000 \\
5.000 \\
11.000 \\
5.000 \\
5.000 \\
11.000 \\
5.000 \\
5.000 \\
5.000 \\
5.000 \\
5.000 \\
5.000 \\
5.000 \\
5.000 \\
11 / R \\
5.000 \\
11.000 \\
11.000 \\
5.000 \\
5.000 \\
5.000 \\
5.000 \\
5.000 \\
5.000 \\
5.000 \\
11 / R \\
11 / R\end{array}$ & $\begin{array}{l}\mathbf{u} \\
\mathbf{u} \\
\mathbf{u} \\
\mathbf{u} \\
\mathbf{u} \\
\mathbf{u} \\
\mathbf{u} \\
\mathbf{u} \\
\mathbf{u} \\
\mathbf{u} \\
\mathbf{u} \\
\mathbf{u} \\
\mathbf{u} \\
\mathbf{u} \\
\mathbf{u} \\
\mathbf{u} \\
\mathbf{u} \\
\mathbf{u} \\
\mathbf{u} \\
\mathbf{u} \\
\mathbf{u} \\
\mathbf{u} \\
\mathbf{u} \\
\mathbf{u} \\
\mathbf{u} \\
\mathbf{u} \\
\mathbf{u} \\
\mathbf{u} \\
\mathbf{u} \\
\mathbf{u} \\
\mathbf{u} \\
\mathbf{u} \\
\mathbf{u} \\
\mathbf{u}\end{array}$ & $\begin{array}{r}11.000 \\
11.000 \\
11.000 \\
11.000 \\
8.000 \\
11.000 \\
5.000 \\
5.000 \\
5.000 \\
5.000 \\
5.000 \\
5.000 \\
11.000 \\
5.000 \\
5.000 \\
11.000 \\
5.000 \\
5.000 \\
5.000 \\
5.000 \\
5.000 \\
5.000 \\
5.000 \\
5.000 \\
11 / 8 \\
5.000 \\
11.000 \\
11.000 \\
5.000 \\
5.000 \\
5.000 \\
5.000 \\
5.000 \\
5.000 \\
5.000 \\
11 / R \\
11 / 8\end{array}$ & $\begin{array}{c}u \\
u \\
u \\
u \\
u s \\
u \\
u \\
u \\
u \\
u \\
u \\
u \\
u \\
u \\
u \\
u \\
u \\
u \\
u \\
u \\
u \\
u \\
u \\
u \\
u \\
u \\
u \\
u \\
u \\
u \\
u \\
u \\
u \\
u \\
u\end{array}$ & $\begin{array}{r}11.000 \\
11.000 \\
11.000 \\
11.000 \\
11.000 \\
11.000 \\
5.000 \\
5.000 \\
5.000 \\
5.000 \\
5.000 \\
5.000 \\
11.000 \\
5.000 \\
5.000 \\
11.000 \\
5.000 \\
5.000 \\
5.000 \\
5.000 \\
5.000 \\
5.000 \\
5.000 \\
5.000 \\
11 / 8 \\
5.000 \\
11.000 \\
11.000 \\
5.000 \\
5.000 \\
5.000 \\
5.000 \\
5.000 \\
5.000 \\
5.000 \\
11 / 2 \\
11 / 2\end{array}$ & $\begin{array}{l}\mathbf{u} \\
\mathbf{u} \\
\mathbf{u} \\
\mathbf{u} \\
\mathbf{u} \\
\mathbf{u} \\
\mathbf{u} \\
\mathbf{u} \\
\mathbf{u} \\
\mathbf{u} \\
\mathbf{u} \\
\mathbf{u} \\
\mathbf{u} \\
\mathbf{u} \\
\mathbf{u} \\
\mathbf{u} \\
\mathbf{u} \\
\mathbf{u} \\
\mathbf{u} \\
\mathbf{u} \\
\mathbf{u} \\
\mathbf{u} \\
\mathbf{u} \\
\mathbf{u} \\
\mathbf{u} \\
\mathbf{u} \\
\mathbf{u} \\
\mathbf{u} \\
\mathbf{u} \\
\mathbf{u} \\
\mathbf{u} \\
\mathbf{u} \\
\mathbf{u} \\
\mathbf{u}\end{array}$ & $\begin{array}{c}11.000 \\
11.000 \\
11.000 \\
11.000 \\
26.000 \\
61.000 \\
5.000 \\
5.000 \\
5.000 \\
5.000 \\
5.000 \\
5.000 \\
11.000 \\
5.000 \\
5.000 \\
11.000 \\
5.000 \\
5.000 \\
5.000 \\
5.000 \\
5.000 \\
5.000 \\
5.000 \\
5.000 \\
11 / 8 \\
5.000 \\
11.000 \\
11.000 \\
5.000 \\
5.000 \\
2.000 \\
5.000 \\
5.000 \\
5.000 \\
5.000 \\
\\
11 / 8 \\
11 / 8\end{array}$ & 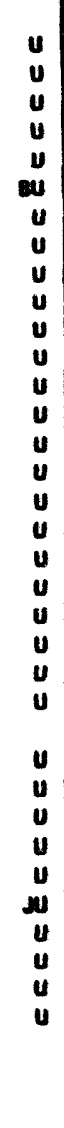 & $\begin{array}{r}11.000 \\
11.000 \\
11.000 \\
11.000 \\
10.000 \\
17.000 \\
5.000 \\
5.000 \\
5.000 \\
5.000 \\
5.000 \\
5.000 \\
11.000 \\
5.000 \\
5.000 \\
11.000 \\
5.000 \\
5.000 \\
5.000 \\
5.000 \\
5.000 \\
5.000 \\
5.000 \\
5.000 \\
11 / 2 \\
5.000 \\
11.000 \\
11.000 \\
5.000 \\
5.000 \\
5.000 \\
5.000 \\
5.000 \\
5.000 \\
5.000 \\
11 / 2 \\
11 / 1\end{array}$ & $\begin{array}{c}u \\
u \\
u \\
u \\
u \\
u \\
u \\
u \\
u \\
u \\
u \\
u \\
u \\
u \\
u \\
u \\
u \\
u \\
u \\
u \\
u \\
u \\
u \\
u \\
u \\
u \\
u \\
u \\
u \\
u \\
u \\
u \\
u \\
u\end{array}$ \\
\hline
\end{tabular}


Socation $307 \mathrm{~T}-3,399-3-17$

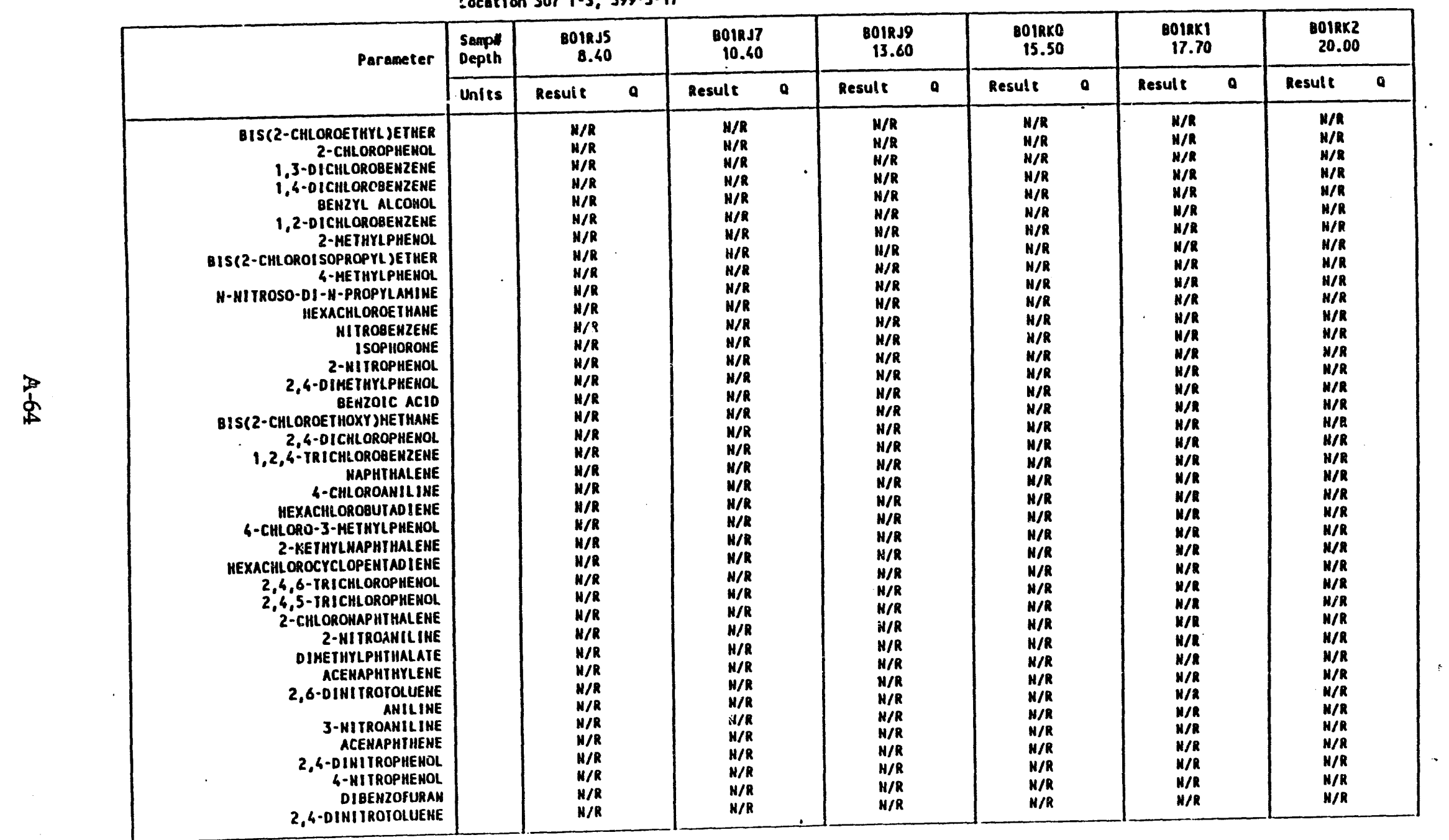


Location 307 r-3, 399-3-17

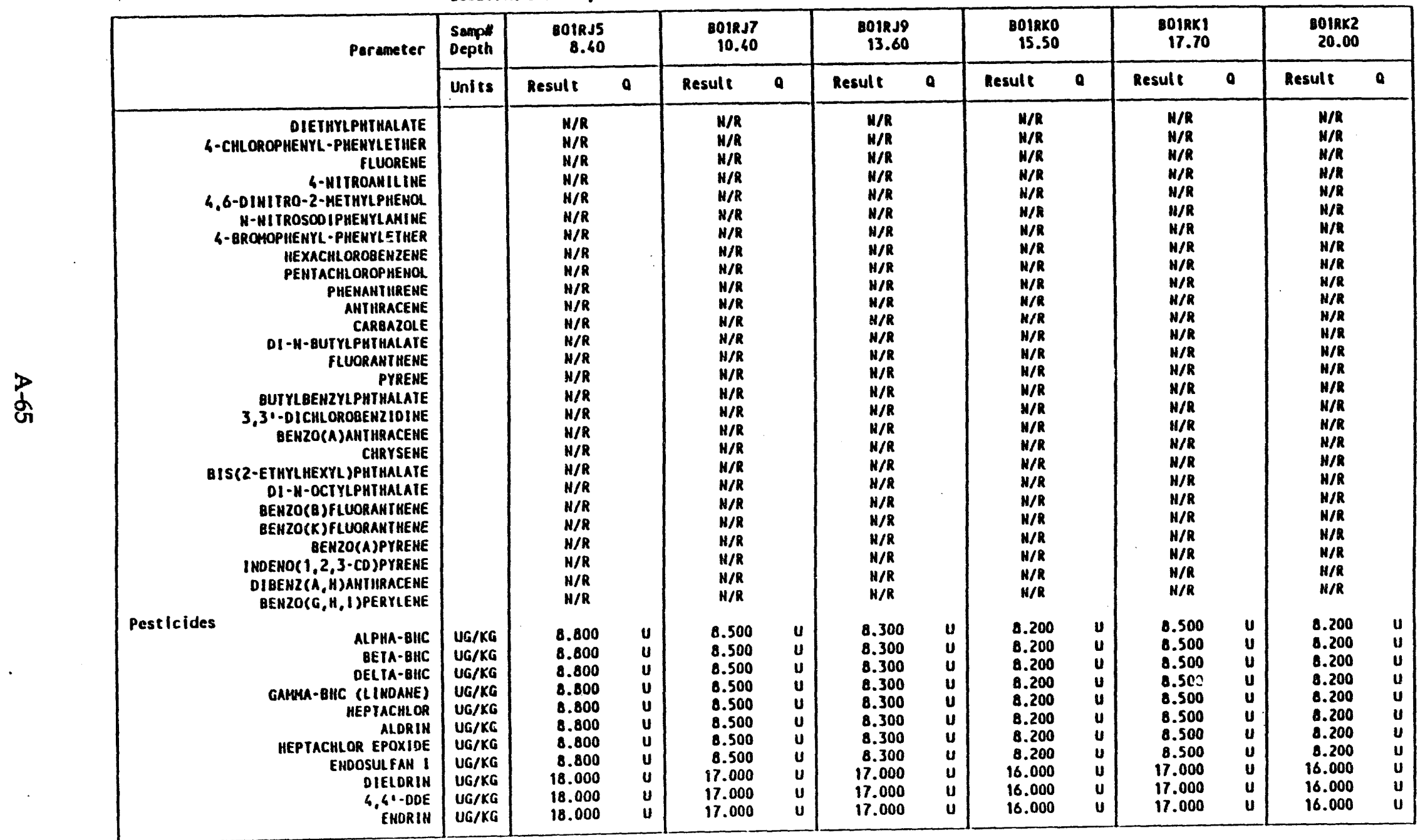


Location 307 r-3, 399-3-17

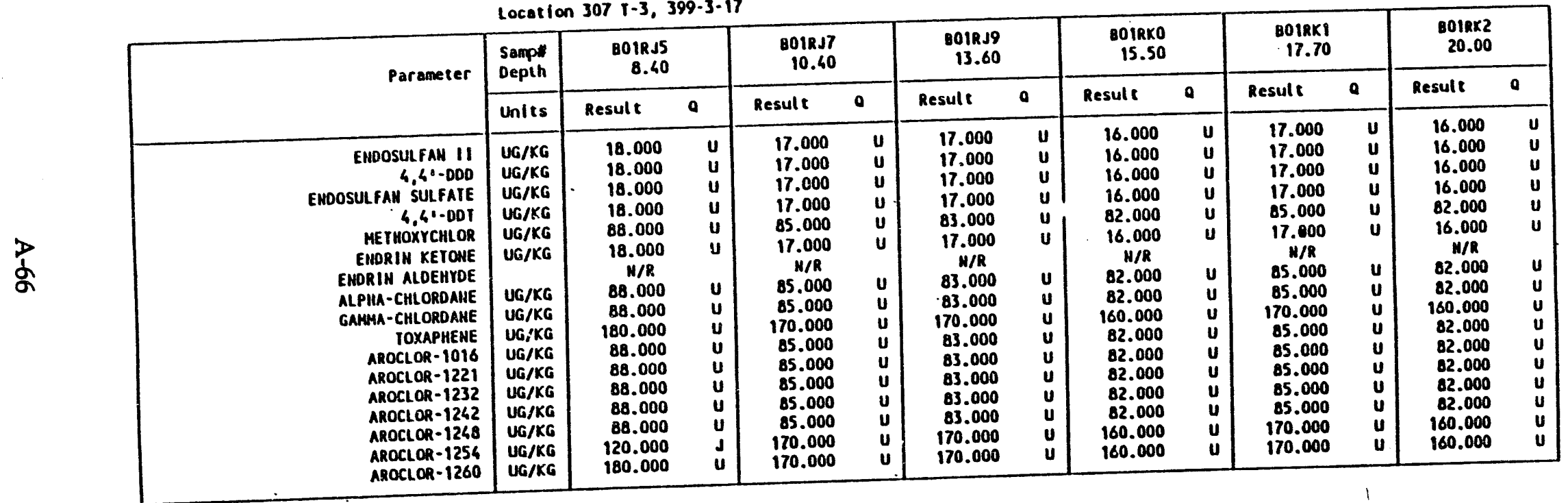




\begin{tabular}{|c|c|c|c|c|c|c|c|c|c|c|c|c|c|}
\hline \multirow[t]{2}{*}{ Paraneter } & \multirow{2}{*}{$\frac{\begin{array}{l}\text { Sampl } \\
\text { Depth }\end{array}}{\text { Units }}$} & \multicolumn{2}{|c|}{$\begin{array}{r}\text { BO1RK3 } \\
22.00\end{array}$} & \multicolumn{2}{|l|}{$\begin{array}{r}\text { B01RKG } \\
24.00\end{array}$} & \multicolumn{2}{|l|}{$\begin{array}{r}\text { 8012k55 } \\
27.30\end{array}$} & \multicolumn{2}{|l|}{$\begin{array}{r}\text { 8018K6 } \\
33.00\end{array}$} & \multicolumn{2}{|l|}{$\begin{array}{r}01212 \times 7 \\
30.00\end{array}$} & \multicolumn{2}{|c|}{$\begin{array}{r}8018 K 9 \\
52.00\end{array}$} \\
\hline & & Result & $\mathbf{a}$ & Result & $a$ & Result & $a$ & Result & a & Result & 0 & Result & a \\
\hline 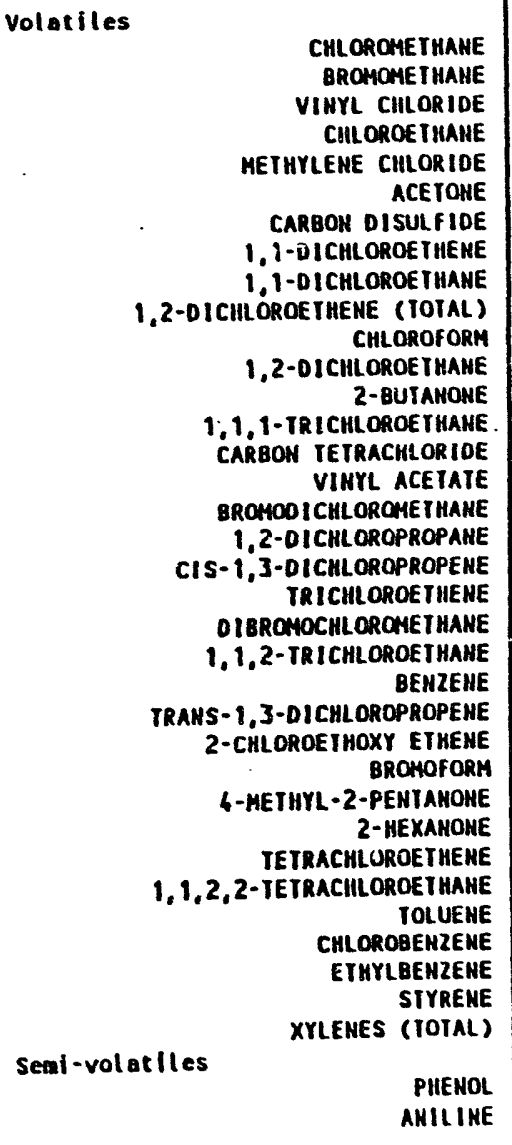 & $\begin{array}{l}U G / K G \\
U G / K G \\
U G / K G \\
U G / K G \\
U G / K G \\
U G / K G \\
U G / K G \\
U G / K G \\
U G / K G \\
U G / K G \\
U G / K G \\
U G / K G \\
U G / K G \\
U G / K G \\
U G / K G \\
U G / K G \\
U G / K G \\
U G / K G \\
U G / K G \\
U G / K G \\
U G / K G \\
U G / K G \\
U G / K G \\
U G / K G \\
U G / K G \\
U G / K G \\
U G / K G \\
U G / K G \\
U G / K G \\
U G / K G \\
U G / K G \\
U G / K G \\
U G / K G \\
U G / K G\end{array}$ & $\begin{array}{r}11.000 \\
11.000 \\
11.000 \\
11.000 \\
11.000 \\
11.000 \\
5.000 \\
5.000 \\
5.000 \\
5.000 \\
5.000 \\
5.000 \\
11.000 \\
5.000 \\
5.000 \\
11.000 \\
5.000 \\
5.000 \\
5.000 \\
5.000 \\
5.000 \\
5.000 \\
5.000 \\
5.000 \\
H / R \\
5.000 \\
11.000 \\
11.000 \\
5.000 \\
5.000 \\
5.000 \\
5.000 \\
5.000 \\
5.000 \\
5.000 \\
W / R \\
H / R\end{array}$ & 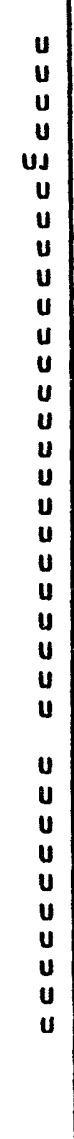 & $\begin{array}{c}11.000 \\
11.000 \\
11.000 \\
11.000 \\
10.000 \\
11.000 \\
5.000 \\
5.000 \\
5.000 \\
5.000 \\
5.000 \\
5.000 \\
11.000 \\
5.000 \\
5.000 \\
11.000 \\
5.000 \\
5.000 \\
5.000 \\
5.000 \\
5.000 \\
5.000 \\
5.000 \\
5.000 \\
1 / R \\
5.000 \\
11.000 \\
11.000 \\
5.000 \\
5.000 \\
5.000 \\
5.000 \\
5.000 \\
5.000 \\
5.000 \\
H / R \\
H / R\end{array}$ & $\begin{array}{l}\mathbf{u} \\
\mathbf{u} \\
\mathbf{u} \\
\mathbf{u} \\
\mathbf{u} \\
\mathbf{u} \\
\mathbf{u} \\
\mathbf{u} \\
\mathbf{u} \\
\mathbf{u} \\
\mathbf{u} \\
\mathbf{u} \\
\mathbf{u} \\
\mathbf{u} \\
\mathbf{u} \\
\mathbf{u} \\
\mathbf{u} \\
\mathbf{u} \\
\mathbf{u} \\
\mathbf{u} \\
\mathbf{u} \\
\mathbf{u} \\
\mathbf{u} \\
\mathbf{u} \\
\mathbf{u} \\
\mathbf{u} \\
\mathbf{u} \\
\mathbf{u} \\
\mathbf{u} \\
\mathbf{u} \\
\mathbf{u} \\
\mathbf{u} \\
\mathbf{u} \\
\mathbf{u}\end{array}$ & $\begin{array}{c}10.000 \\
10.000 \\
10.000 \\
10.000 \\
13.000 \\
10.000 \\
5.000 \\
5.000 \\
5.000 \\
5.000 \\
5.000 \\
5.000 \\
10.000 \\
5.000 \\
5.000 \\
10.000 \\
5.000 \\
5.000 \\
5.000 \\
5.000 \\
5.000 \\
5.000 \\
5.000 \\
5.000 \\
11 / R \\
5.000 \\
10.000 \\
10.000 \\
5.000 \\
5.000 \\
5.000 \\
5.000 \\
5.000 \\
5.000 \\
5.000 \\
\\
H 1 / R \\
H / R \\
\end{array}$ & $\begin{array}{c}u \\
u \\
u \\
u \\
u s \\
u \\
u \\
u \\
u \\
u \\
u \\
u \\
u \\
u \\
u \\
u \\
u \\
u \\
u \\
u \\
u \\
u \\
u \\
u \\
u \\
u \\
u \\
u \\
u \\
u \\
u \\
u \\
u \\
u\end{array}$ & $\begin{array}{c}11.000 \\
11.000 \\
11.000 \\
11.000 \\
13.000 \\
11.000 \\
5.000 \\
5.000 \\
5.000 \\
5.000 \\
5.000 \\
5.000 \\
11.000 \\
5.000 \\
5.000 \\
11.000 \\
5.000 \\
5.000 \\
5.000 \\
5.000 \\
5.000 \\
5.000 \\
5.000 \\
5.000 \\
H / R \\
5.000 \\
11.000 \\
11.000 \\
5.000 \\
5.000 \\
5.000 \\
5.000 \\
5.000 \\
5.000 \\
5.000 \\
H / R \\
H / R \\
\end{array}$ & $\begin{array}{c}u \\
u \\
u \\
u \\
u s \\
u \\
u \\
u \\
u \\
u \\
u \\
u \\
u \\
u \\
u \\
u \\
u \\
u \\
u \\
u \\
u \\
u \\
u \\
u \\
u \\
u \\
u \\
u \\
u \\
u \\
u \\
u \\
u \\
v\end{array}$ & $\begin{array}{c}11.000 \\
11.000 \\
11.000 \\
11.000 \\
10.000 \\
11.000 \\
5.000 \\
5.000 \\
5.000 \\
5.000 \\
5.000 \\
5.000 \\
11.000 \\
5.000 \\
5.000 \\
11.000 \\
5.000 \\
5.000 \\
5.000 \\
5.000 \\
5.000 \\
5.000 \\
5.000 \\
5.000 \\
11 / R \\
5.000 \\
11.000 \\
11.000 \\
5.000 \\
5.000 \\
5.000 \\
5.000 \\
5.000 \\
5.000 \\
5.000 \\
1 \% / R \\
1 / R \\
\end{array}$ & $\begin{array}{c}u \\
u \\
u \\
u \\
u s \\
u \\
u \\
u \\
u \\
u \\
u \\
u \\
u \\
u \\
u \\
u \\
u \\
u \\
u \\
u \\
u \\
u \\
u \\
u \\
u \\
u \\
u \\
u \\
u \\
u \\
u \\
u \\
u \\
u \\
v\end{array}$ & $\begin{array}{c}11.000 \\
11.000 \\
11.000 \\
11.000 \\
14.000 \\
10.000 \\
6.000 \\
6.000 \\
6.000 \\
6.000 \\
6.000 \\
6.000 \\
11.000 \\
6.000 \\
6.000 \\
11.000 \\
6.000 \\
6.000 \\
6.000 \\
6.000 \\
6.000 \\
6.000 \\
6.000 \\
6.000 \\
11 / R \\
6.000 \\
11.000 \\
11.000 \\
6.000 \\
6.000 \\
6.000 \\
6.000 \\
6.000 \\
6.000 \\
6.000 \\
\\
11 / R \\
1 / R\end{array}$ & $\begin{array}{l}u \\
u \\
u \\
u \\
u \\
u u \\
u \\
u \\
u \\
u \\
u \\
u \\
u \\
u \\
u \\
u \\
u \\
u \\
u \\
u \\
u \\
u \\
u \\
u \\
u \\
u \\
u \\
u \\
u \\
u \\
u \\
u \\
u \\
u\end{array}$ \\
\hline
\end{tabular}


Locat Ion $307 \mathrm{I}-3,399-3-17$

\begin{tabular}{|c|c|c|c|c|c|c|c|c|c|c|c|}
\hline \multirow[t]{2}{*}{ Paraneter } & $\begin{array}{l}\text { Sempll } \\
\text { Depth }\end{array}$ & $\begin{array}{r}8018 \times 3 \\
22.00\end{array}$ & \multicolumn{2}{|c|}{$\begin{array}{r}8018 K 4 \\
24.00\end{array}$} & $\begin{array}{r}8018 \times 5 \\
27.30\end{array}$ & \multicolumn{2}{|c|}{$\begin{array}{r}\text { 801RK6 } \\
33.00\end{array}$} & \multicolumn{2}{|c|}{$\begin{array}{r}\text { Bo1RK7 } \\
38.00\end{array}$} & \multicolumn{2}{|c|}{$\begin{array}{r}2017 \times K 9 \\
52.00\end{array}$} \\
\hline & Units & Result & Result & $a$ & Result & Result & 0 & Result & $\mathbf{a}$ & Result & $a$ \\
\hline
\end{tabular}


Location 307 7-3, 399-3-17

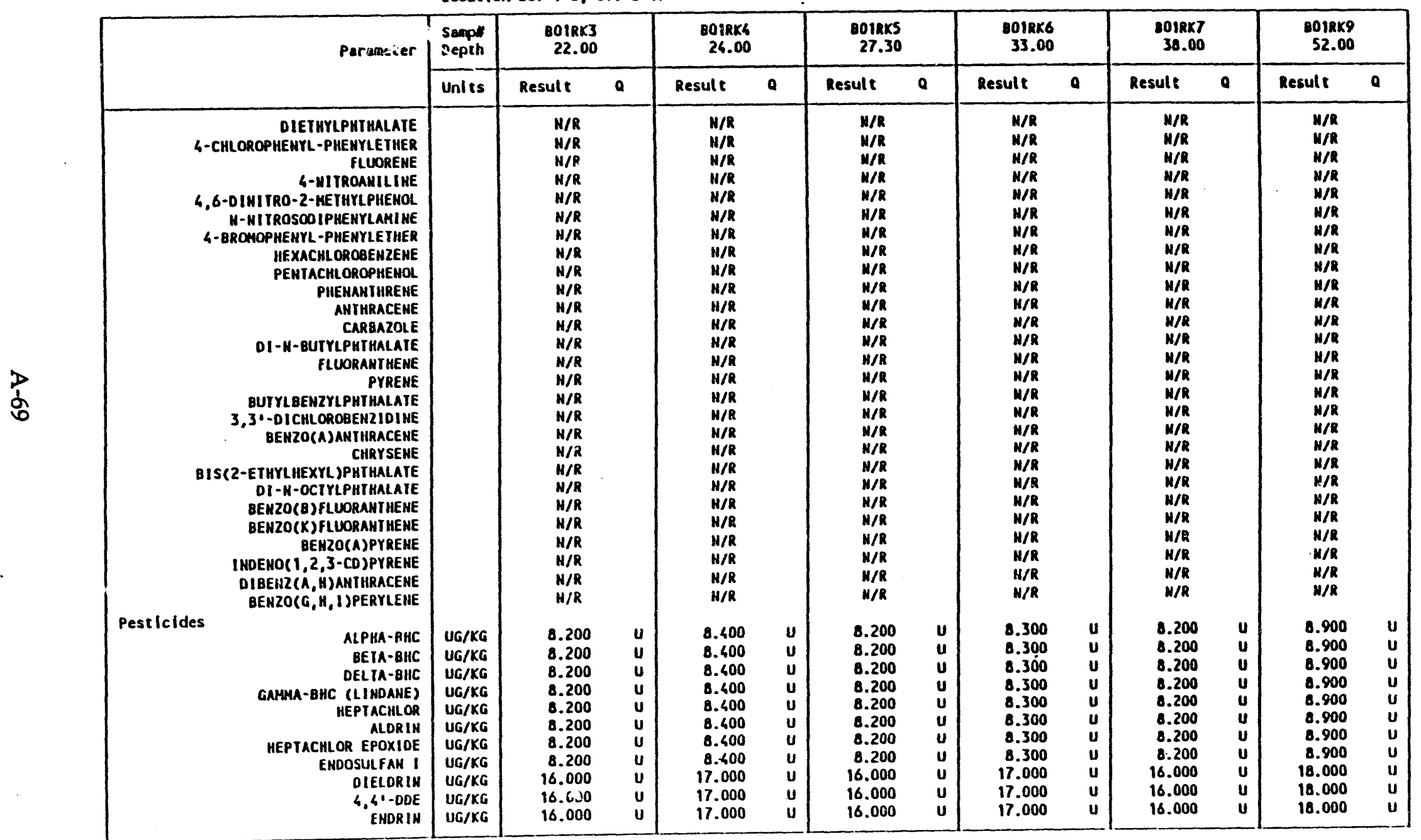


Location 307 1-3, 399-3-17

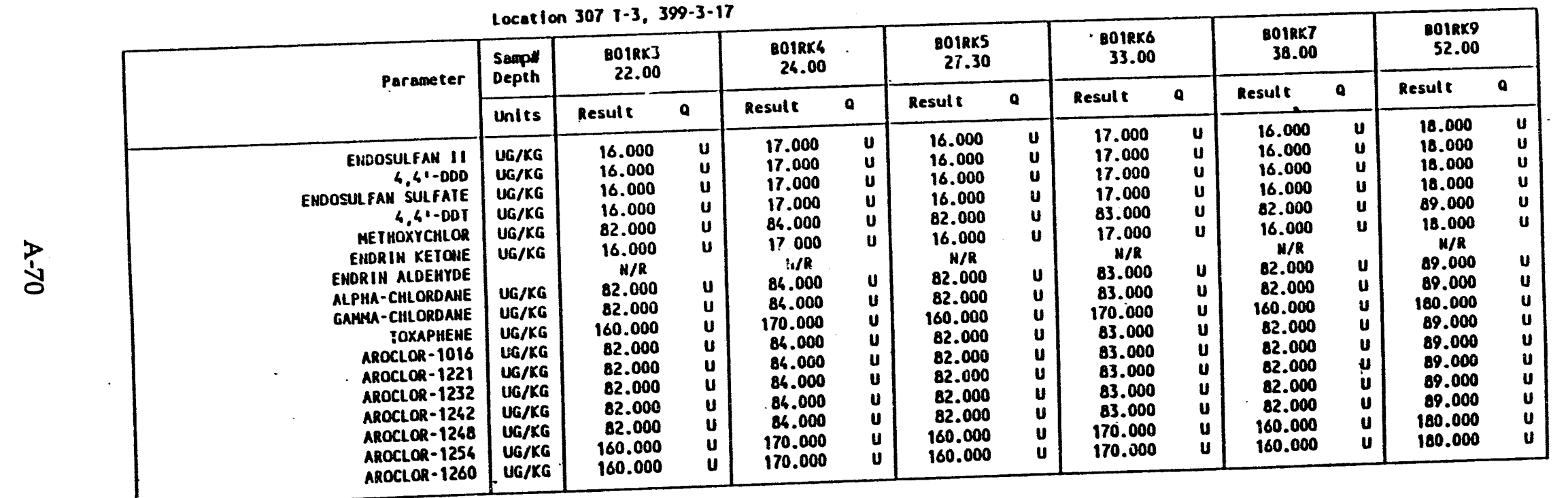


WHC-SD-EN-TI-279, Rev. 0

Location 307 T-3, 399-3-17

\begin{tabular}{|c|c|c|c|}
\hline \multirow[t]{2}{*}{ Paraneter } & \multirow{2}{*}{$\frac{\begin{array}{c}\text { Sampi } \\
\text { Depth }\end{array}}{\text { Units }}$} & \multicolumn{2}{|l|}{$\begin{array}{r}\text { B01RKs } \\
65.00\end{array}$} \\
\hline & & Resule & 9 \\
\hline 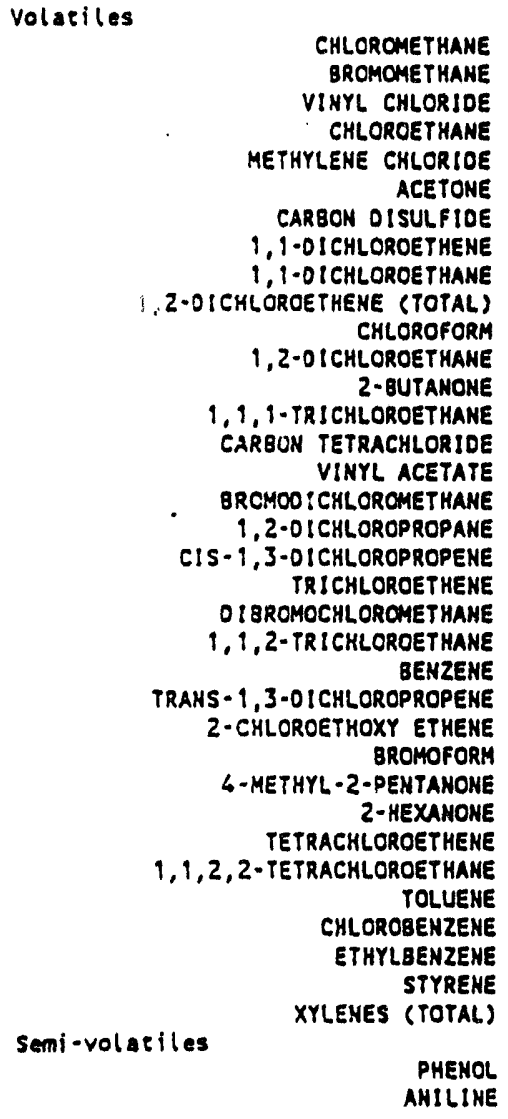 & $\begin{array}{l}\text { UG/KG } \\
U G / K G \\
U G / K G \\
U G / K G \\
U G / K G \\
U G / K G \\
U G / K G \\
U G / K G \\
U G / K G \\
U G / K G \\
U G / K G \\
U G / K G \\
U G / K G \\
U G / K G \\
U G / K G \\
U G / K G \\
U G / K G \\
U G / K G \\
U G / K G \\
U G / K G \\
U G / K G \\
U G / K G \\
U G / K G \\
U G / K G \\
U G / K G \\
U G / K G \\
U G / K G \\
U G / K G \\
U G / K G \\
U G / K G \\
U G / K G \\
U G / K G \\
U G / K G \\
U G / K G\end{array}$ & $\begin{array}{r}11.000 \\
11.000 \\
11.000 \\
11.000 \\
11.000 \\
10.000 \\
6.000 \\
6.000 \\
6.000 \\
6.000 \\
6.000 \\
6.000 \\
11.000 \\
6.000 \\
6.000 \\
11.000 \\
6.000 \\
6.000 \\
6.000 \\
6.000 \\
6.000 \\
6.000 \\
6.000 \\
6.000 \\
1 / R \\
6.000 \\
11.000 \\
11.000 \\
6.000 \\
6.000 \\
6.000 \\
6.000 \\
6.000 \\
6.000 \\
6.000 \\
\\
11 / R \\
11 / 2\end{array}$ & $\begin{array}{l}U \\
U \\
U \\
U \\
U \\
U J \\
U \\
U \\
U \\
U \\
U \\
U \\
U \\
U \\
U \\
U \\
U \\
U \\
U \\
U \\
U \\
U \\
U \\
U \\
U \\
U \\
U \\
U \\
U \\
U \\
U \\
U \\
U \\
U \\
U\end{array}$ \\
\hline
\end{tabular}


WHC-SD-EN-TI-279, Rev. 0

This page left intentionally blank. 
Location 307 7-1, 399-3-15

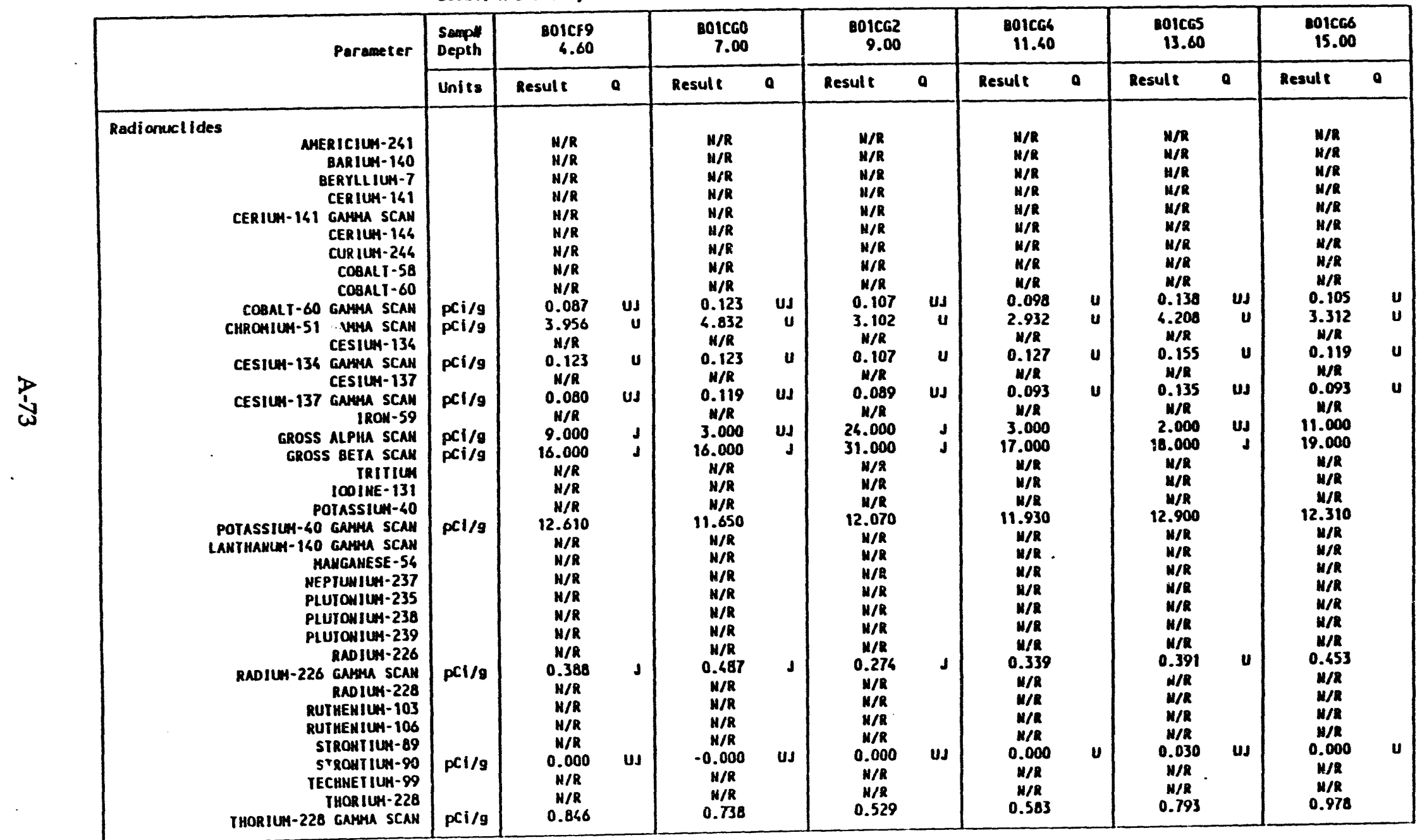




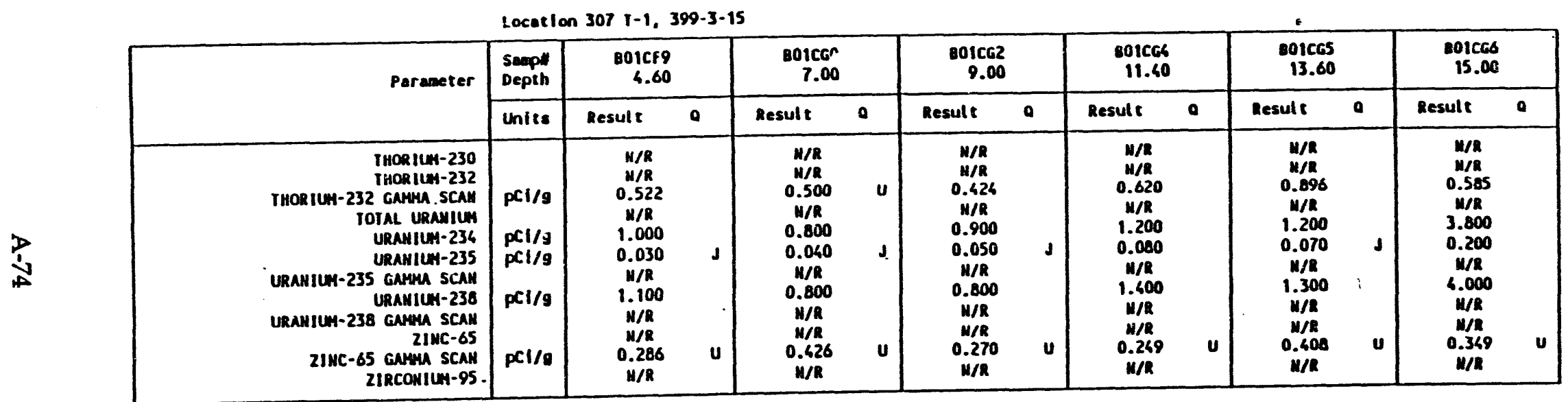


Locat Ion 307 7-1, 399-3-15

\begin{tabular}{|c|c|c|c|c|c|c|c|c|c|c|c|c|c|}
\hline \multirow[t]{2}{*}{ Paraseter } & \multirow{2}{*}{$\begin{array}{l}\text { Samps" } \\
\text { Depth }\end{array}$} & \multicolumn{2}{|l|}{$\begin{array}{r}801 C G 7 \\
17.00 \\
\end{array}$} & \multicolumn{2}{|l|}{$\begin{array}{r}801 c c 80 \\
20.00\end{array}$} & \multicolumn{2}{|l|}{$\begin{array}{r}801 C G 9 \\
22.50\end{array}$} & \multicolumn{2}{|l|}{$\begin{array}{r}\text { Bolcuo } \\
26.50\end{array}$} & \multicolumn{2}{|l|}{$\begin{array}{l}0.1 c 41 \\
30.00\end{array}$} & \multicolumn{2}{|l|}{$\begin{array}{r}801 \mathrm{CH}^{2} \\
37.00\end{array}$} \\
\hline & & Result & a & Result & 0 & Result & a & Result & a & Result & 0 & Result & a \\
\hline 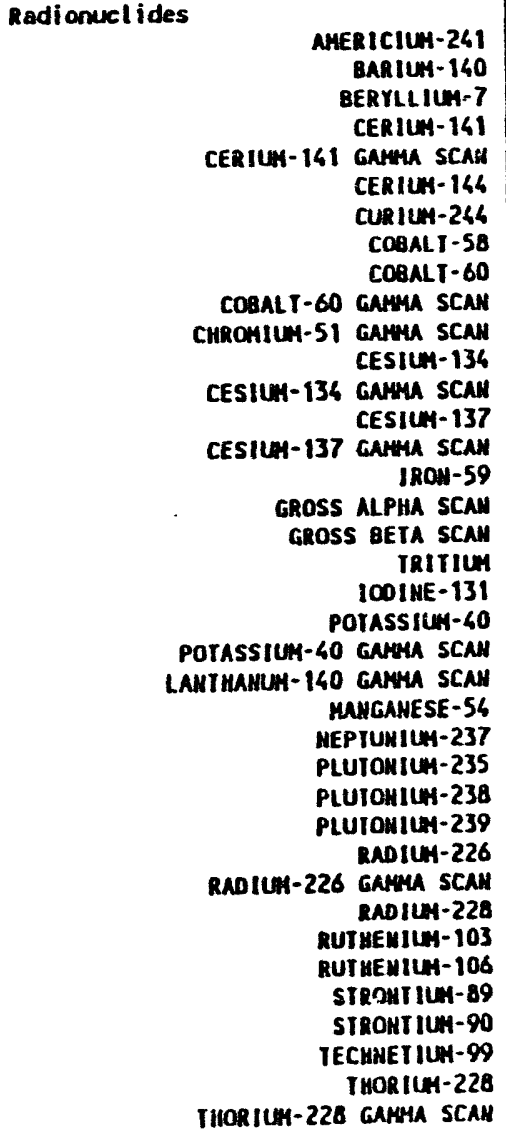 & $\begin{array}{l}\mathrm{pCi} / \mathrm{g} \\
\mathrm{pCi} / \mathrm{g} \\
\mathrm{pCl} / \mathrm{g} \\
\mathrm{pCi} / \mathrm{g} \\
\mathrm{pCl} / \mathrm{g} \\
\mathrm{pCi} / \mathrm{g}\end{array}$ & 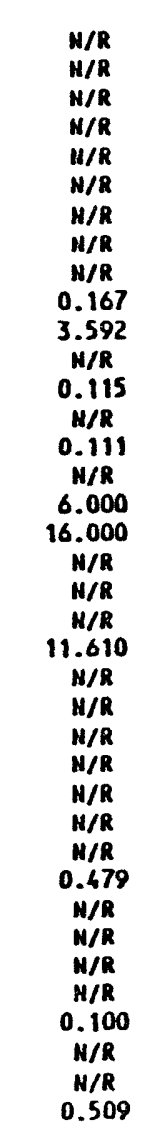 & $\begin{array}{l}\text { u } \\
\mathbf{u} \\
\mathbf{u} \\
\text { us } \\
\mathbf{J} \\
\mathbf{J}\end{array}$ & 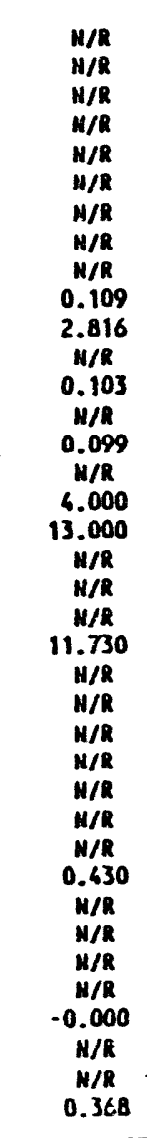 & $\begin{array}{c}\text { UJ } \\
\text { U } \\
\text { U } \\
\text { Uנ } \\
\mathbf{J}\end{array}$ & 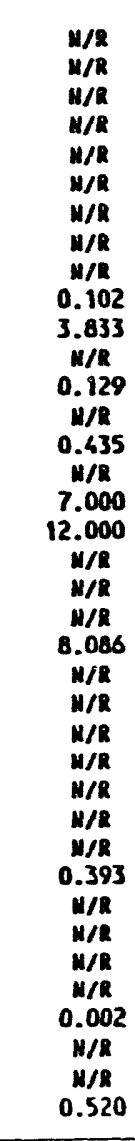 & $\begin{array}{c}\text { UJ } \\
\text { U } \\
\text { U } \\
\mathbf{J} \\
\mathbf{J}\end{array}$ & 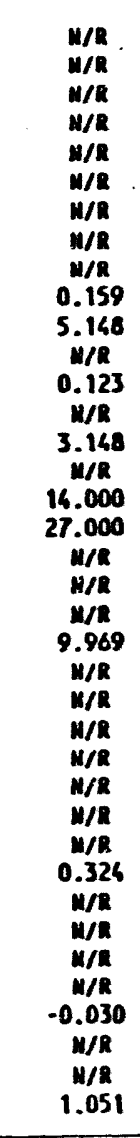 & $\begin{array}{c}\text { UJ } \\
\mathbf{u} \\
\mathbf{u} \\
\mathbf{J} \\
\mathbf{J}\end{array}$ & 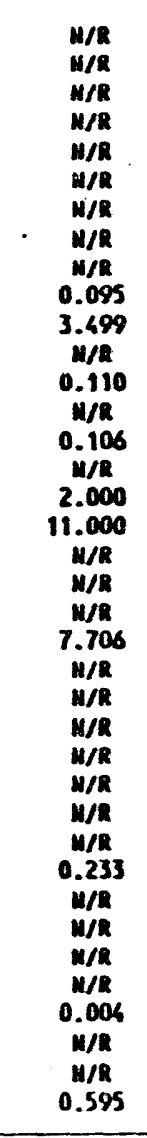 & $\begin{array}{l}u \\
u \\
u \\
u\end{array}$ & 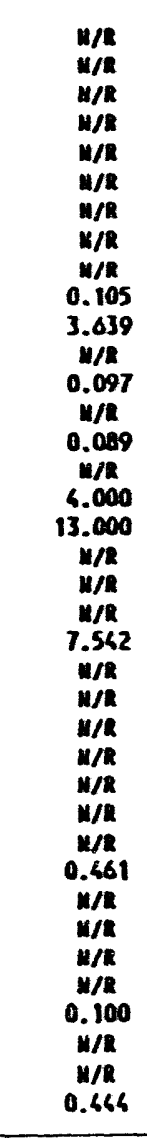 & $\begin{array}{l}u \\
u \\
u \\
u\end{array}$ \\
\hline
\end{tabular}


Location 307 I-1, 399-3-15

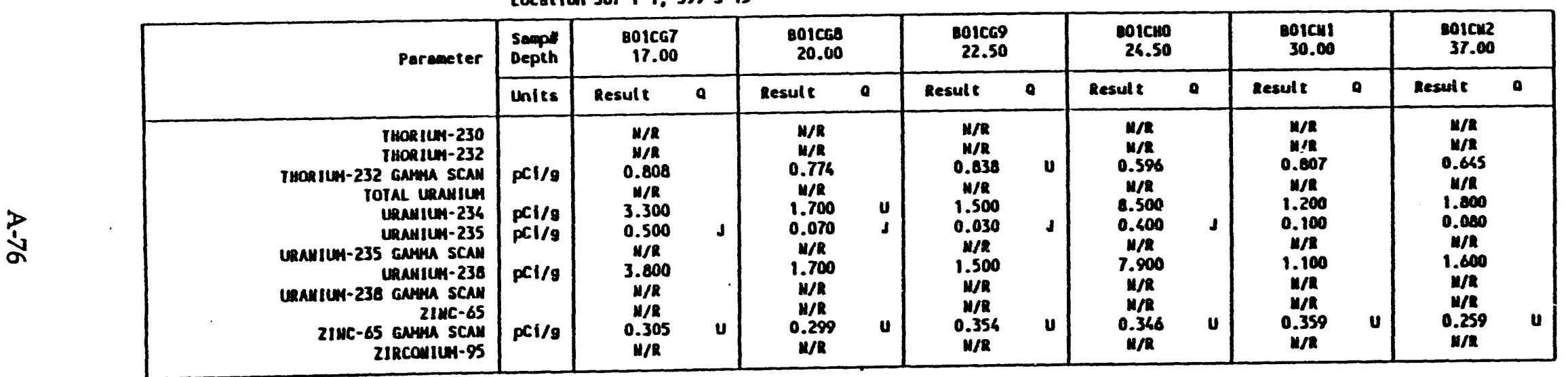




\begin{tabular}{|c|c|c|c|c|c|c|c|c|c|c|c|}
\hline \multirow[t]{2}{*}{ Parameter } & \multirow{2}{*}{$\begin{array}{l}\text { Samp: } \\
\text { Depth }\end{array}$} & \multicolumn{2}{|l|}{$\begin{array}{r}8018 J U \\
42.00\end{array}$} & \multicolumn{2}{|l|}{$\begin{array}{l}\text { BOIRJS } \\
47.00\end{array}$} & \multicolumn{2}{|l|}{$\begin{array}{r}\text { Boins. } \\
53.00\end{array}$} & \multicolumn{2}{|l|}{$\begin{array}{r}\text { Doles] } \\
58.00\end{array}$} & \multicolumn{2}{|c|}{$\begin{array}{l}8012124 \\
68.00\end{array}$} \\
\hline & & Result & 0 & Result & a & Result & a & Result & - & Result & a \\
\hline 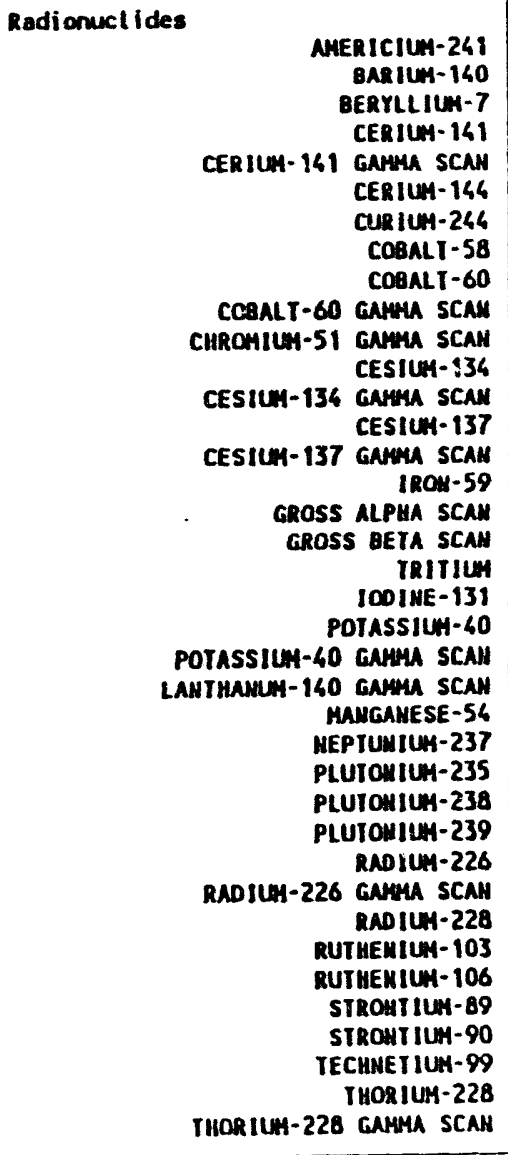 & 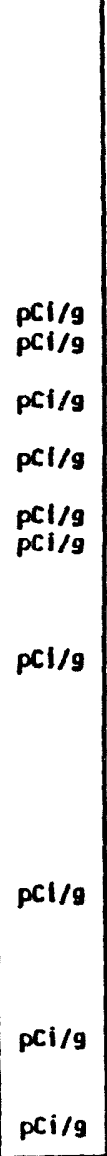 & 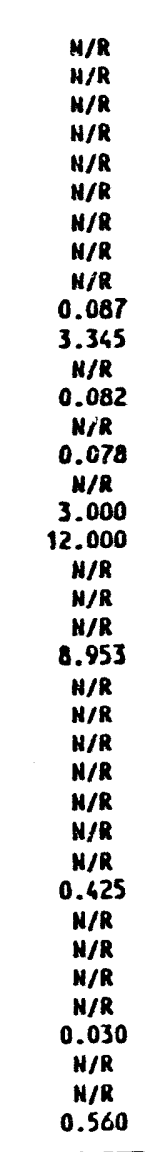 & $\begin{array}{l}u \\
u \\
u \\
u\end{array}$ & 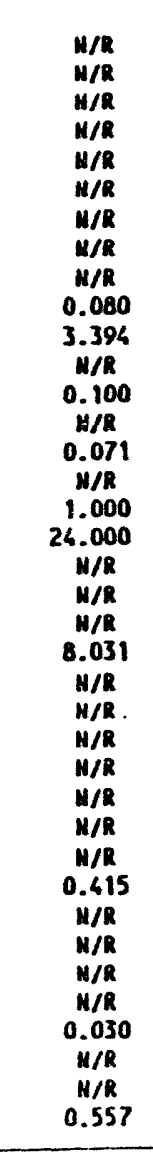 & $\begin{array}{l}u \\
u \\
u \\
u\end{array}$ & 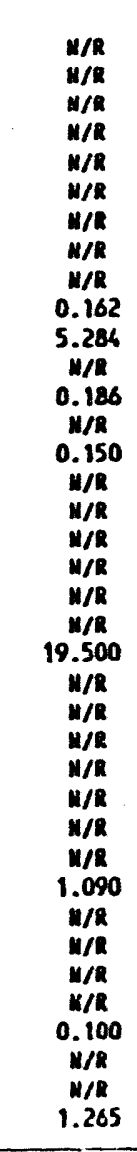 & $\begin{array}{l}u \\
u \\
u \\
u\end{array}$ & 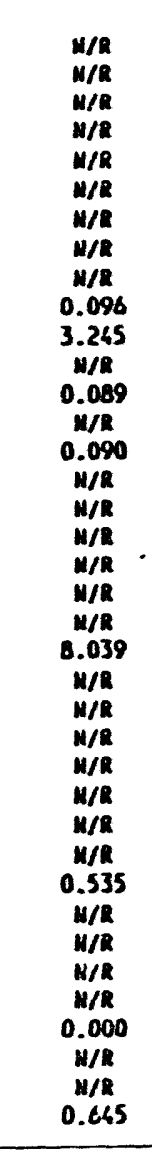 & $\begin{array}{l}u \\
u \\
v \\
v\end{array}$ & 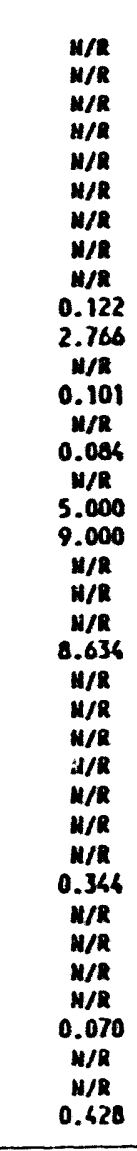 & $\begin{array}{l}\mathbf{u} \\
\mathbf{u} \\
\mathbf{u} \\
\mathbf{u}\end{array}$ \\
\hline
\end{tabular}



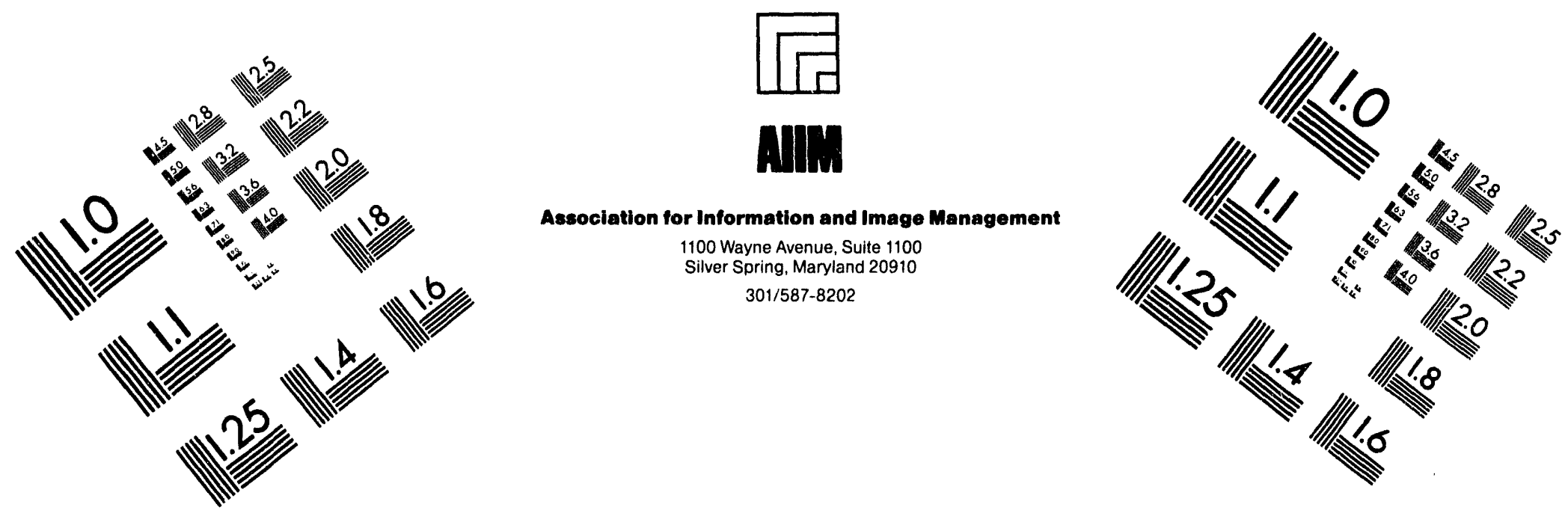

\section{Centimeter}

$\begin{array}{llllllllllllllll}1 & 2 & 3 & 4 & 5 & 6 & 7 & 8 & 9 & 10 & 11 & 12 & 13 & 14 & 15 & \mathrm{~mm}\end{array}$

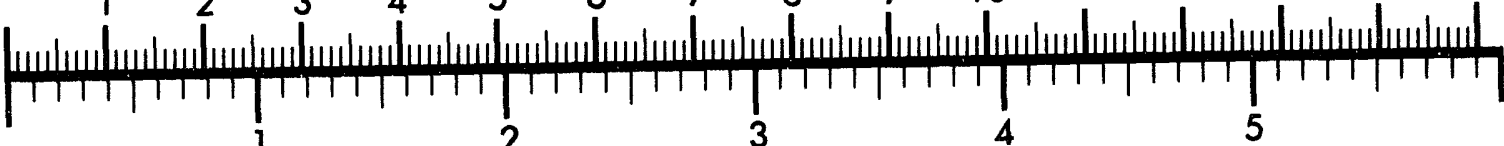

Inches
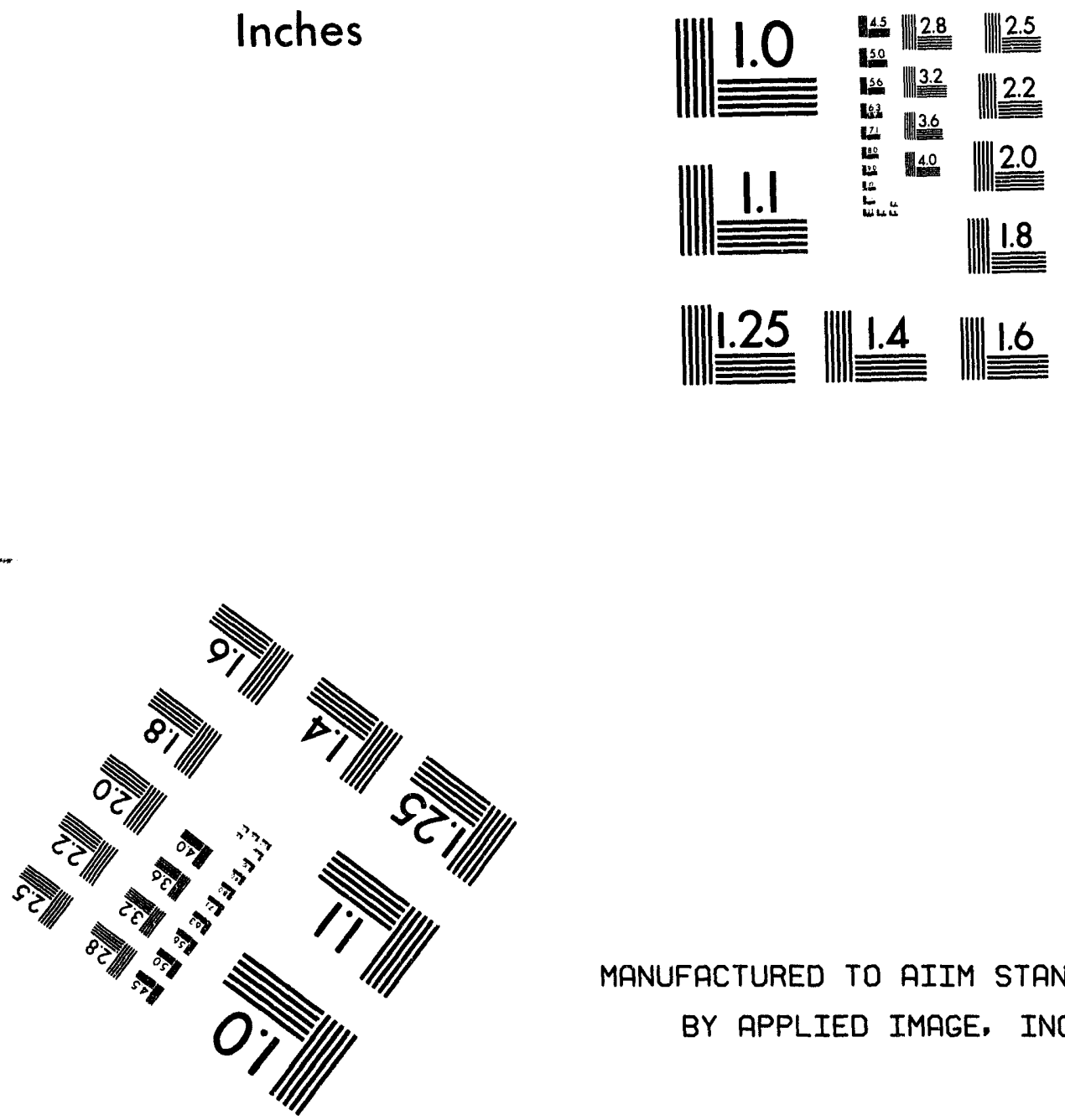

MANUFACTURED TO AIIM STANDARDS

BY APPLIED IMAGE, INC.

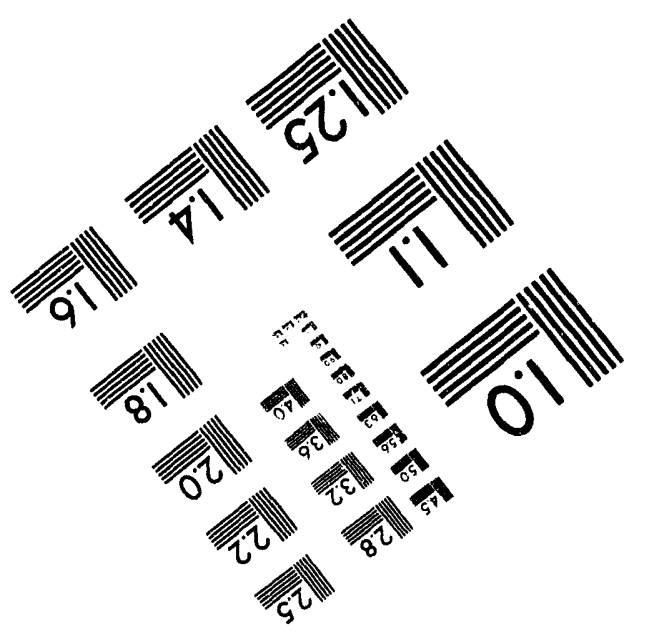



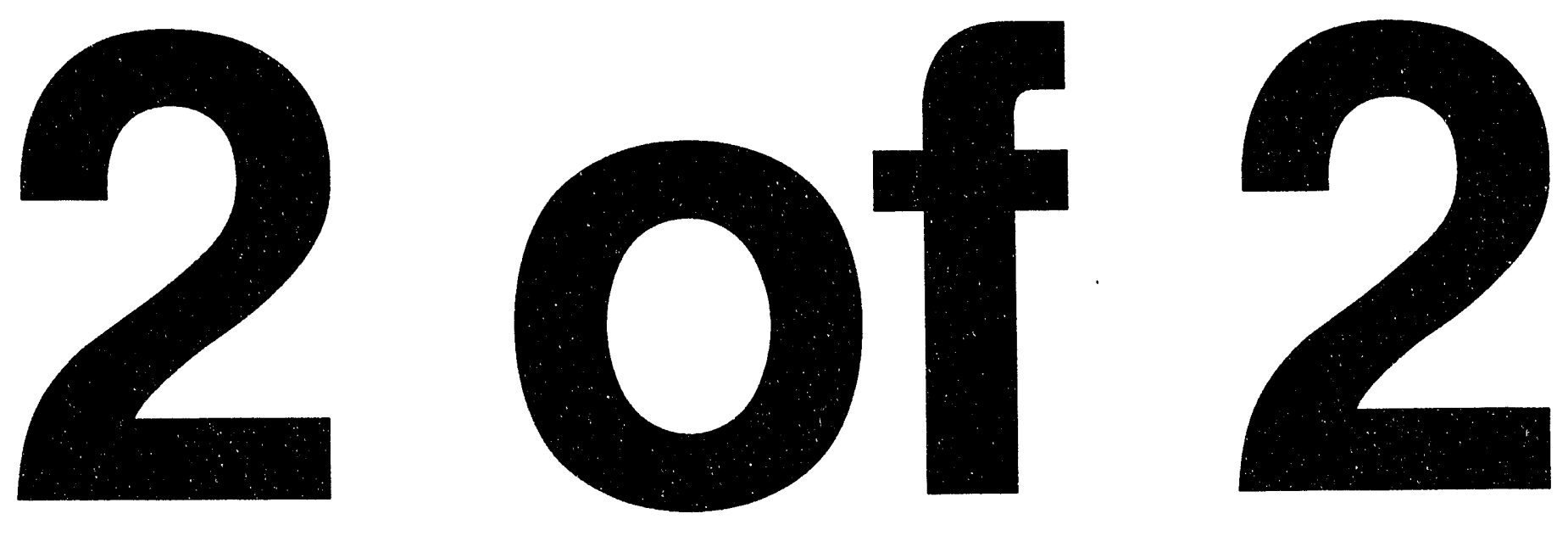


\begin{tabular}{|c|c|c|c|c|c|c|c|c|c|c|c|}
\hline \multirow[t]{2}{*}{ Parameter } & \multirow{2}{*}{\begin{tabular}{|l|}
$\begin{array}{l}\text { Sampot } \\
\text { Depth }\end{array}$ \\
Units \\
\end{tabular}} & \multicolumn{2}{|l|}{$\begin{array}{r}\text { B01RJ0 } \\
42.00\end{array}$} & \multicolumn{2}{|l|}{$\begin{array}{r}\text { B01RJ1 } \\
47.00\end{array}$} & \multicolumn{2}{|l|}{$\begin{array}{r}8018 J 22 \\
53.00\end{array}$} & \multicolumn{2}{|l|}{$\begin{array}{r}01 R J 33 \\
58.00\end{array}$} & \multicolumn{2}{|c|}{$\begin{array}{r}8018.44 \\
68.00\end{array}$} \\
\hline & & Result & $a$ & Result t & $a$ & Result & 0 & Result t & a & Result & 0 \\
\hline 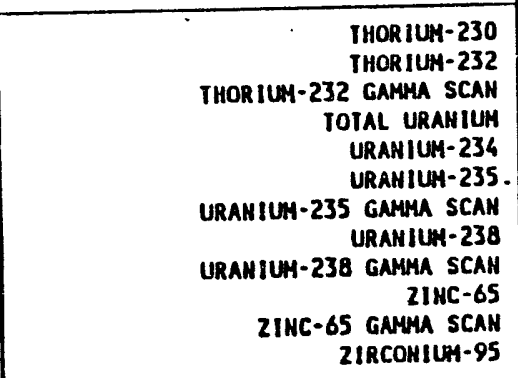 & $\begin{array}{l}\mathrm{pCl} / \mathrm{g} \\
\mathrm{pCl} / \mathrm{g} \\
\mathrm{pCI} / \mathrm{g} \\
\mathrm{pCl} / \mathrm{g} \\
\mathrm{pCl/g}\end{array}$ & $\begin{array}{l}H / R \\
H / R \\
O .517 \\
H / R \\
2.300 \\
0.100 \\
H / R \\
2.400 \\
N / R \\
H / R \\
0.210 \\
H / R\end{array}$ & u & $\begin{array}{l}H / R \\
H / R \\
0.694 \\
H / R \\
2.600 \\
0.100 \\
H / R \\
2.400 \\
H / R \\
H / R \\
0.232 \\
H / R\end{array}$ & u & $\begin{array}{l}K / R \\
H / R \\
0.862 \\
H / R \\
2.800 \\
0.100 \\
H / R \\
2.200 \\
N / R \\
H / R \\
0.501 \\
N / R\end{array}$ & $u$ & $\begin{array}{l}H / R \\
H / R \\
0.638 \\
N / R \\
0.700 \\
0.040 \\
W / R \\
0.700 \\
H / R \\
H / R \\
0.289 \\
H / R\end{array}$ & u & $\begin{array}{c}W / R \\
H / R \\
N / R \\
0.442 \\
H / R \\
0.040 \\
0.200 \\
W / R \\
5.000 \\
W / R \\
H / R \\
0.213 \\
W / R \\
W / R\end{array}$ & 1 \\
\hline
\end{tabular}


WHC-SD-EN-TI-279, Rev. 0

This page left intentionally blank. 
Location 307 I-2, 399-3-16

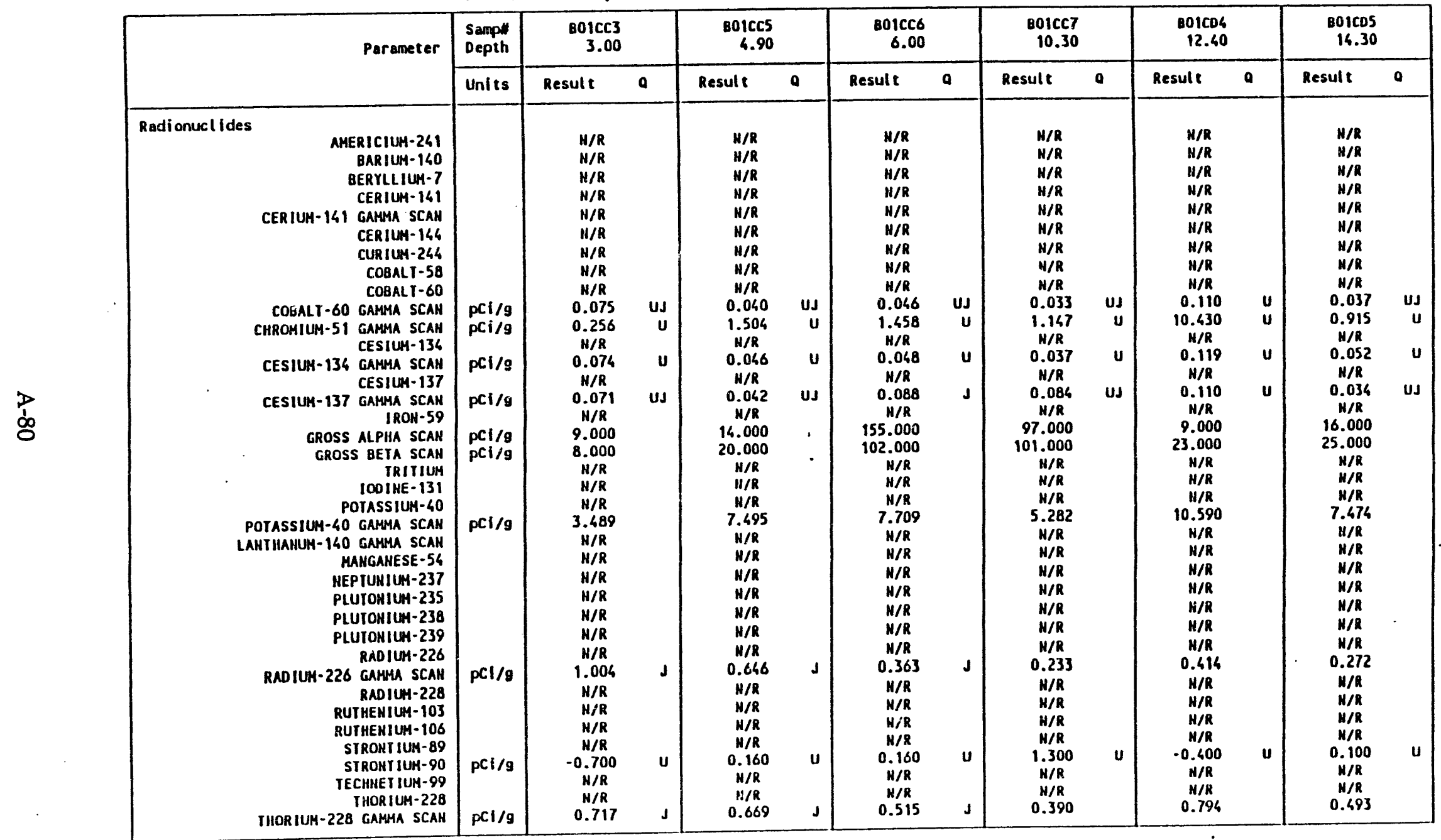


Location 307 7-2, 399-3-16

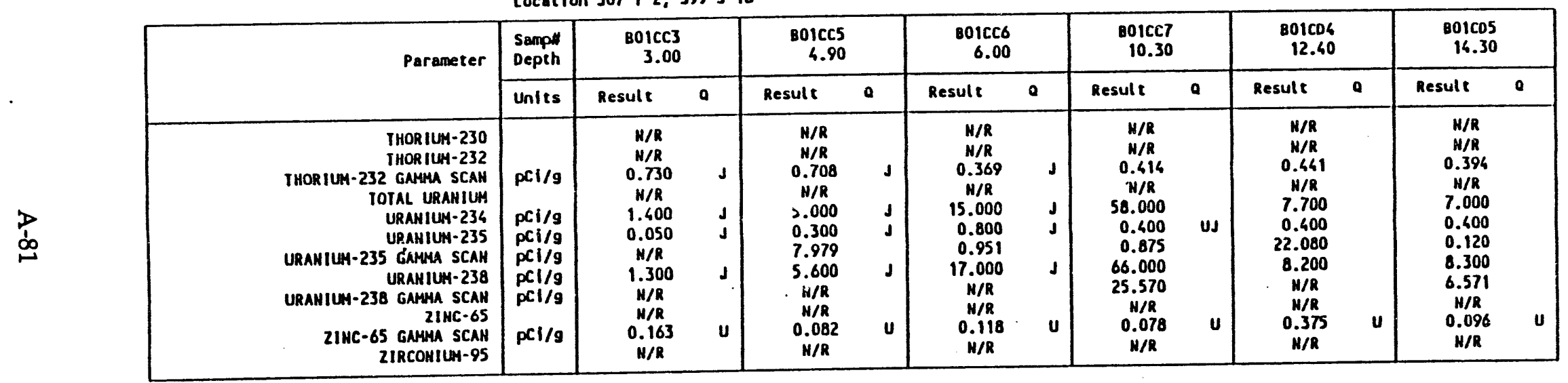


Location 307 T-2, 399-3-16

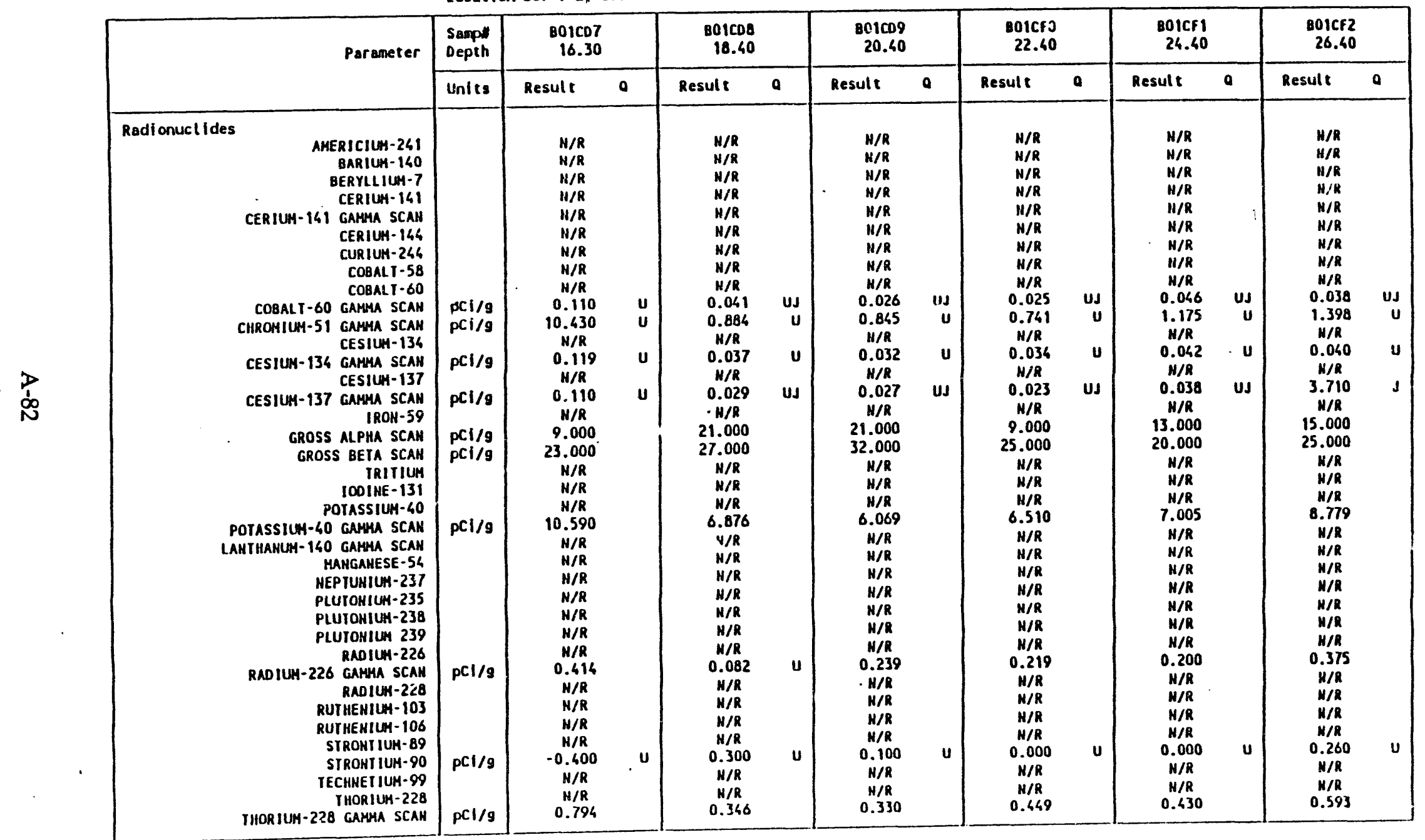




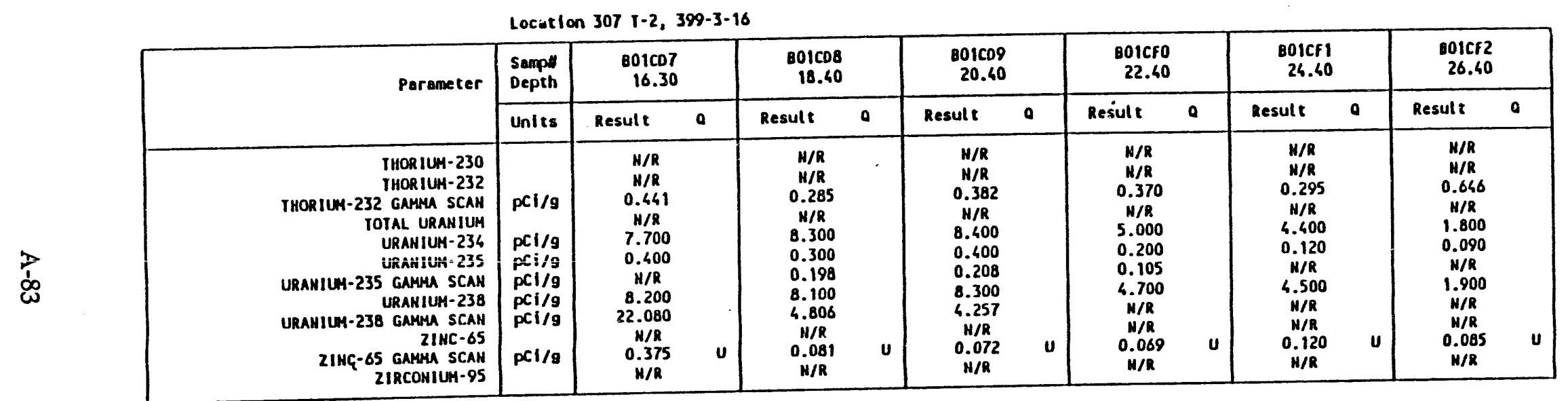


Locat Ion 307 T-2, 399-3-16

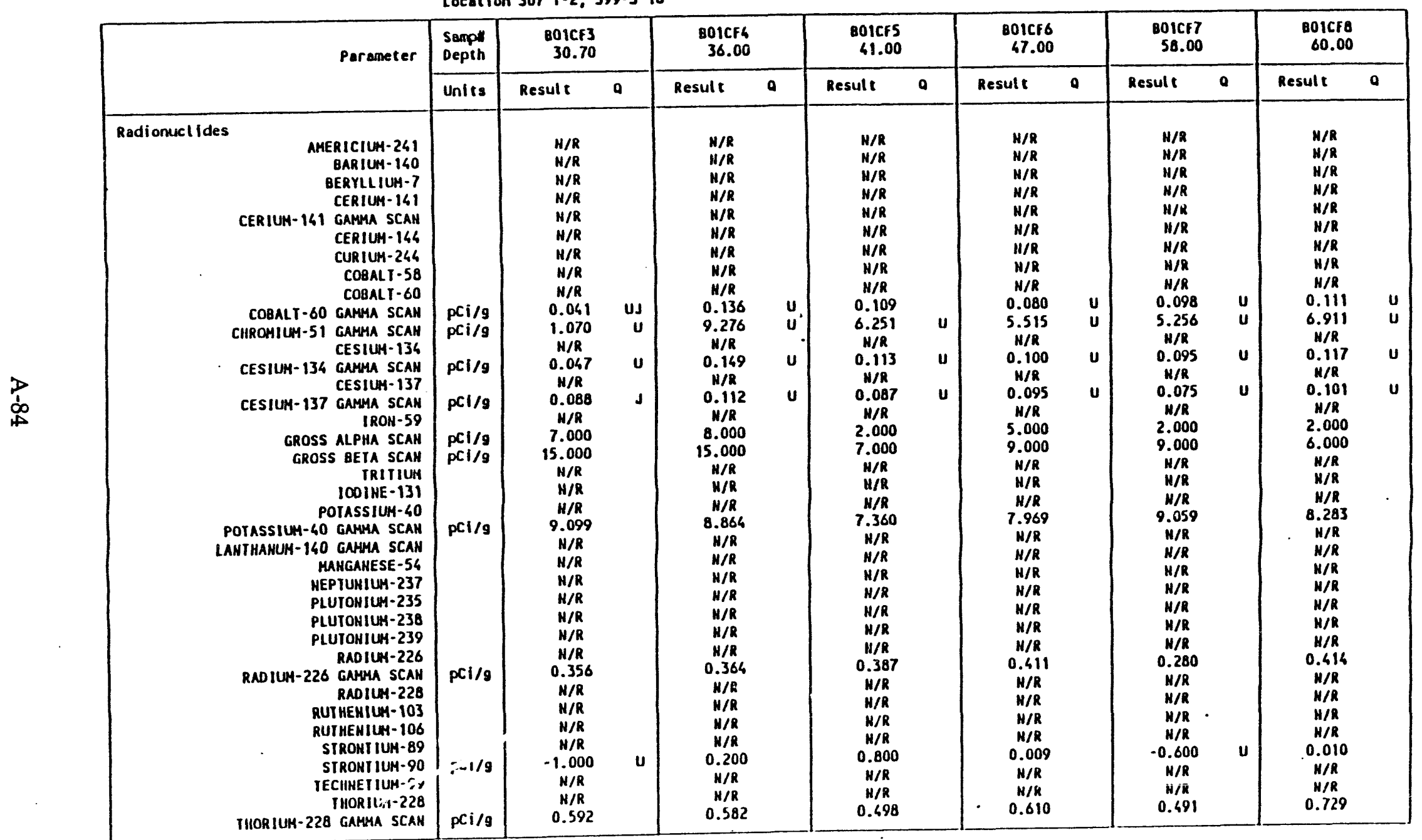


Locat Ion $307 \mathrm{~T}-2$, 399-3-16

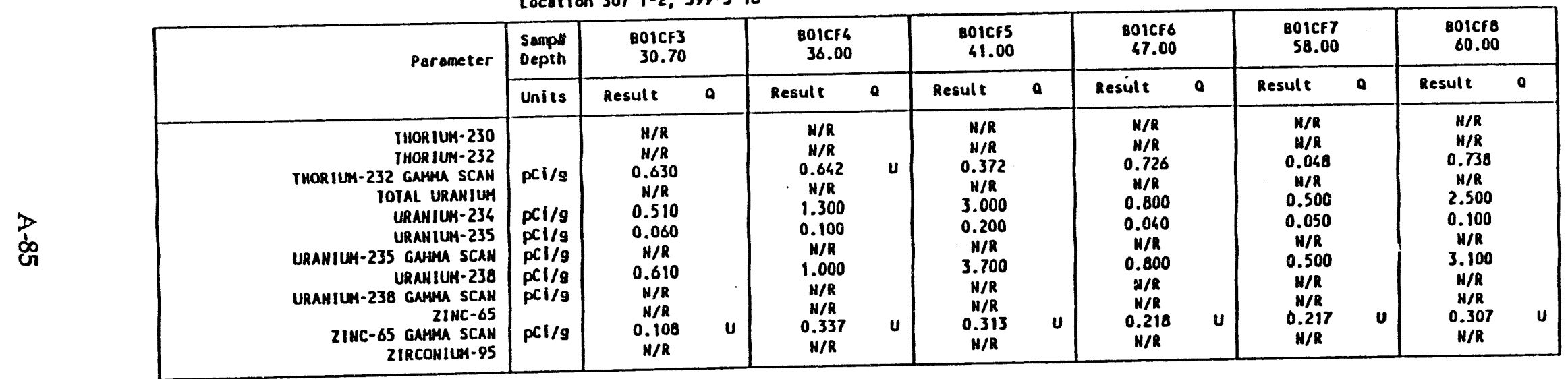


WHC-SD-EN-TI-279, Rev. 0

This page left intentionally blank. 
Location 307 1-3, 399-3-17

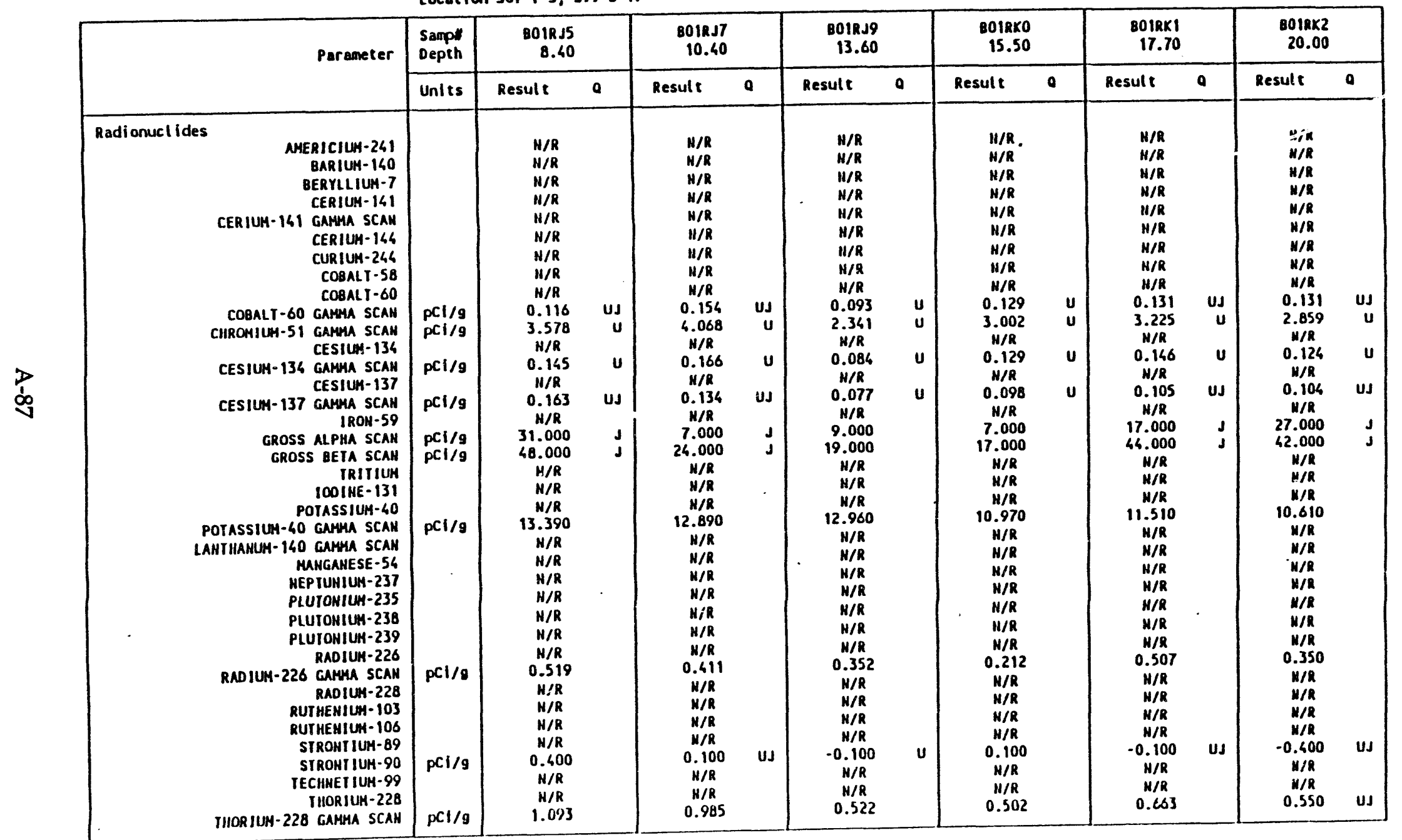


Location 307 I-3, 399-3-17

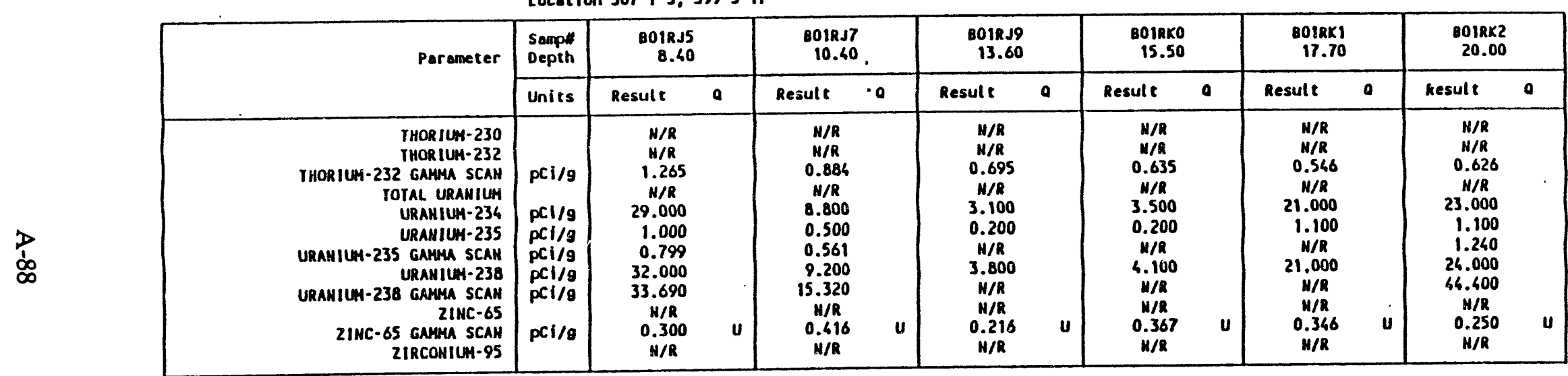


Location 307 7-3, 399-3-17

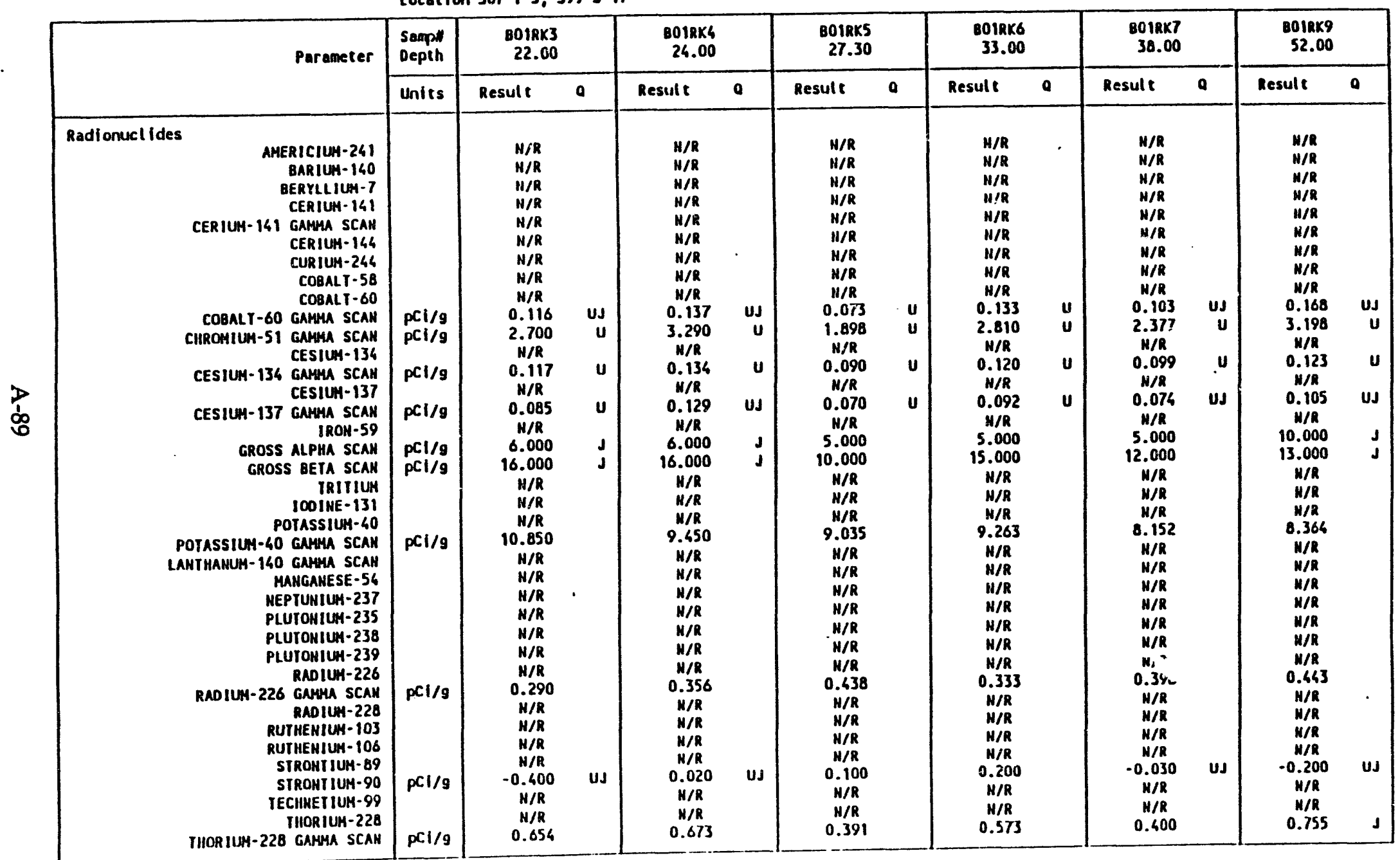


Locat ion 307 I-3, 399-3-17

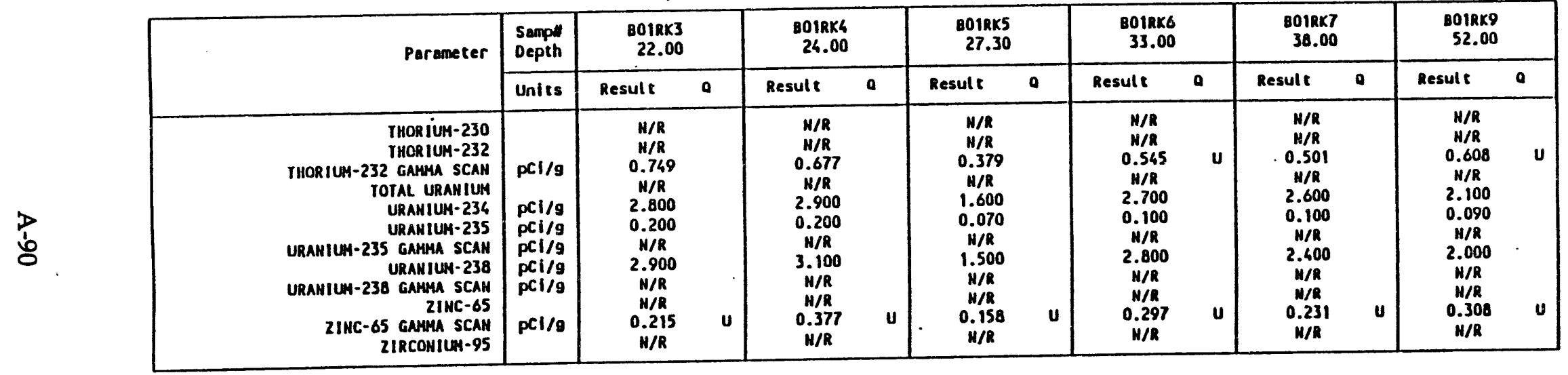


WHC-SD-EN-TI-279, Rev. 0

Location 307 T-3, 399-3-17

\begin{tabular}{|c|c|c|c|}
\hline \multirow[t]{2}{*}{ Parameter } & \multirow{2}{*}{$\frac{\begin{array}{l}\text { Semp: } \\
\text { Oepeh }\end{array}}{\text { Units }}$} & \multicolumn{2}{|l|}{$\begin{array}{r}8018 \times 3 \\
65.00\end{array}$} \\
\hline & & Resule & 0 \\
\hline 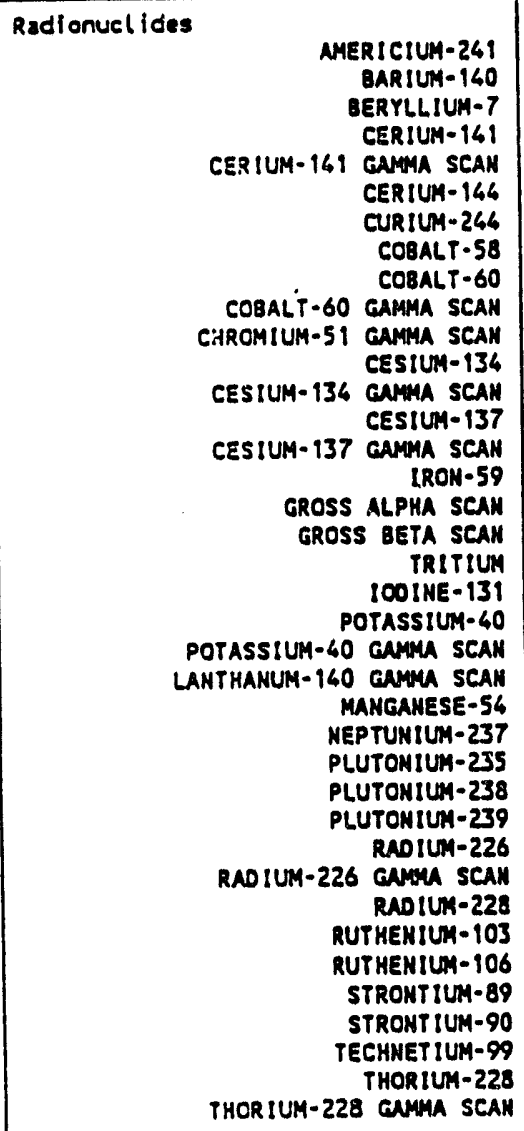 & $\begin{array}{l}p C i / g \\
p C i / g \\
p C i / g \\
p C i / g \\
p C i / g \\
p C i / g\end{array}$ & 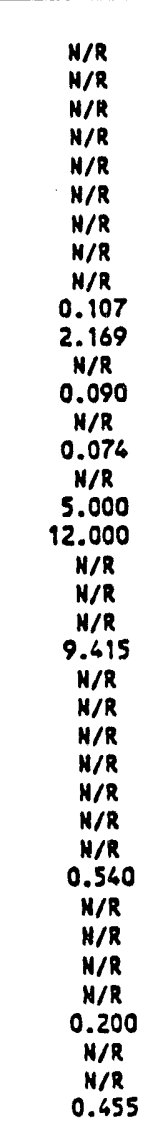 & $\begin{array}{c}\text { UJ } \\
u \\
u \\
\text { uJ } \\
\text { UJ } \\
J\end{array}$ \\
\hline
\end{tabular}


WHC-SD-EN-TI-279, Rev. 0

Location 307 T-3, 399-3-17

\begin{tabular}{|c|c|c|c|}
\hline \multirow[t]{2}{*}{ Parameter } & \multirow{2}{*}{$\begin{array}{l}\text { Sampl } \\
\text { Depeh }\end{array}$} & \multicolumn{2}{|c|}{$\begin{array}{r}8018 \times 8 \\
65.00\end{array}$} \\
\hline & & Resule & 0 \\
\hline $\begin{array}{r}\text { THORIUM-230 } \\
\text { THORIUM-232 } \\
\text { THORIUM-232 GAMMA SCAN } \\
\text { TOTAL URAMIUM } \\
\text { URANIUM-234 } \\
\text { URANIUM-235 } \\
\text { URAMIUM-235 GAMMA SCAN } \\
\text { URANIUM-238 } \\
\text { URANIUM-238 GAMMA SCAN } \\
\text { Z1NC-65 } \\
\text { 2INC-65 GNHMA SCAN } \\
\text { 21RCOMIUM-95 }\end{array}$ & $\begin{array}{l}\mathrm{pCi} / \mathrm{g} \\
\mathrm{pCi} / \mathrm{g} \\
\mathrm{pCi} / \mathrm{g} \\
\mathrm{pCi} / \mathrm{g} \\
\mathrm{pCi} / \mathrm{g} \\
\mathrm{pCi} / \mathrm{g} \\
\mathrm{pCi}\end{array}$ & $\begin{array}{l}N / R \\
N / R \\
0.661 \\
N / R \\
0.500 \\
0.030 \\
N / R \\
0.600 \\
N / R \\
N / R \\
0.206 \\
H / R\end{array}$ & $u$ \\
\hline
\end{tabular}


WHC-SD-EN-TI-279, Rev. 0

This page left intentionally blank. 


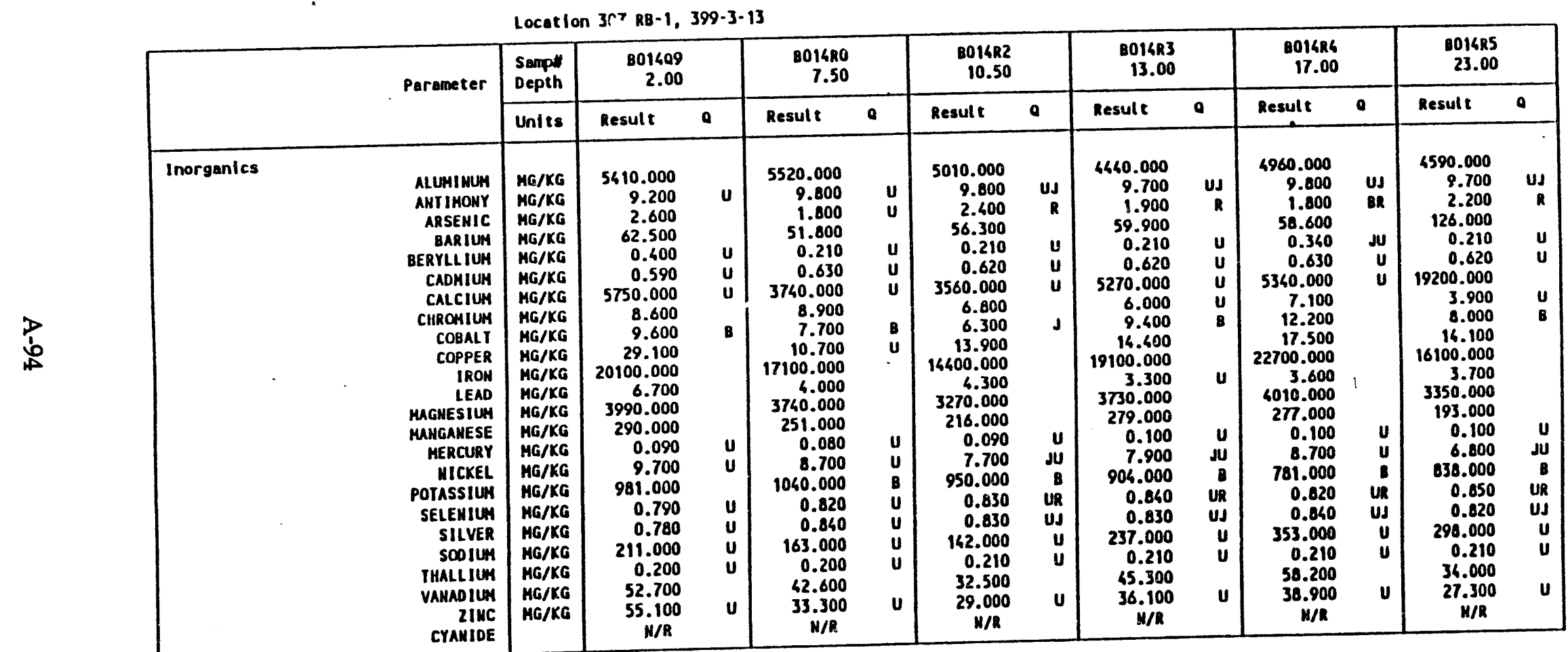




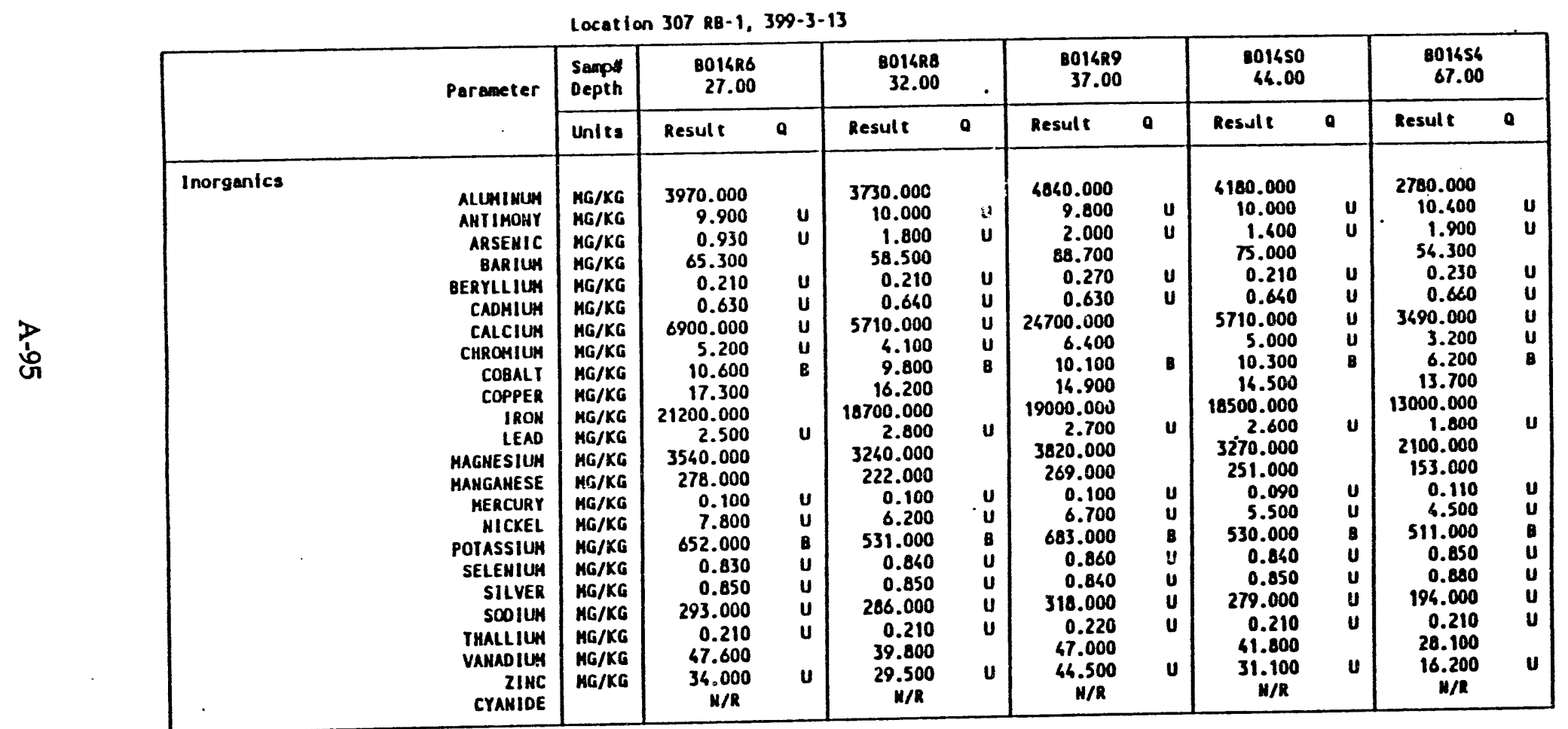


WHC-SD-EN-TI-279, Rev. 0

This page left intentionally blank. 
Location 307 RB-2, 399-3-14

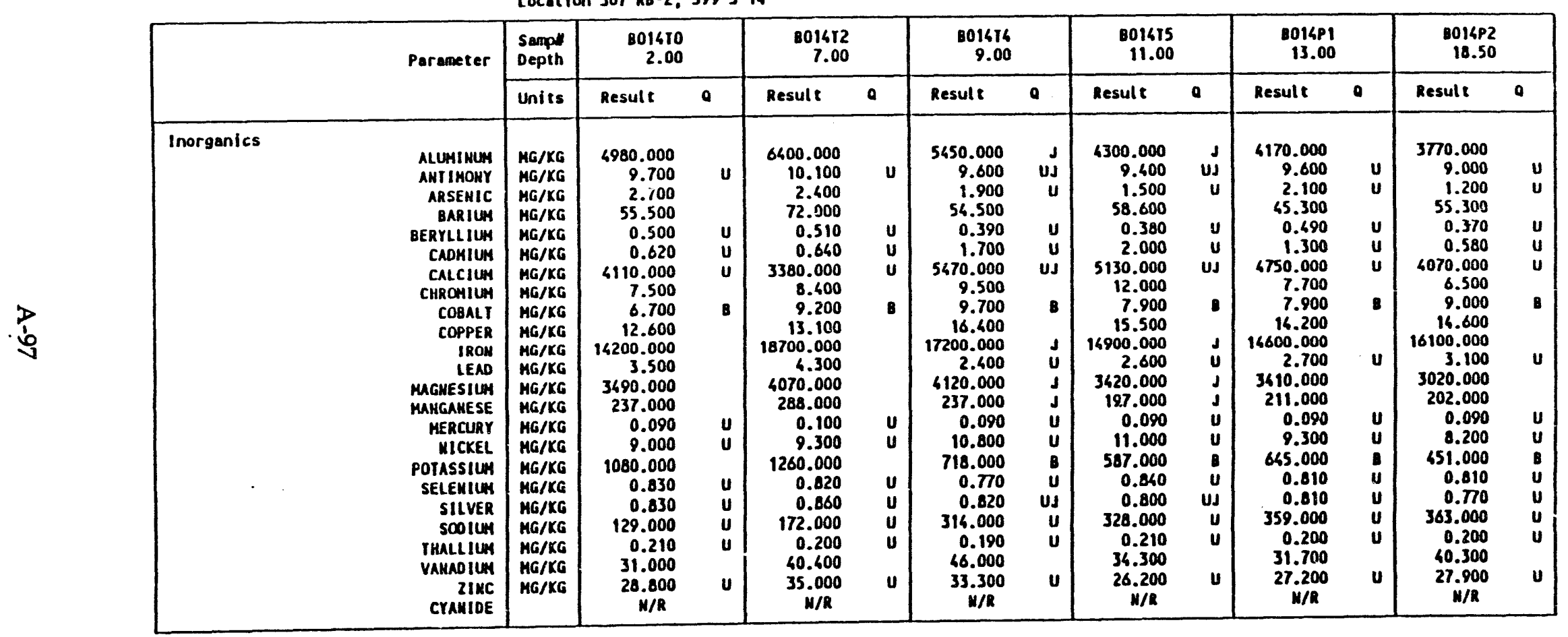


Location 307 RB-2, 399-3-14

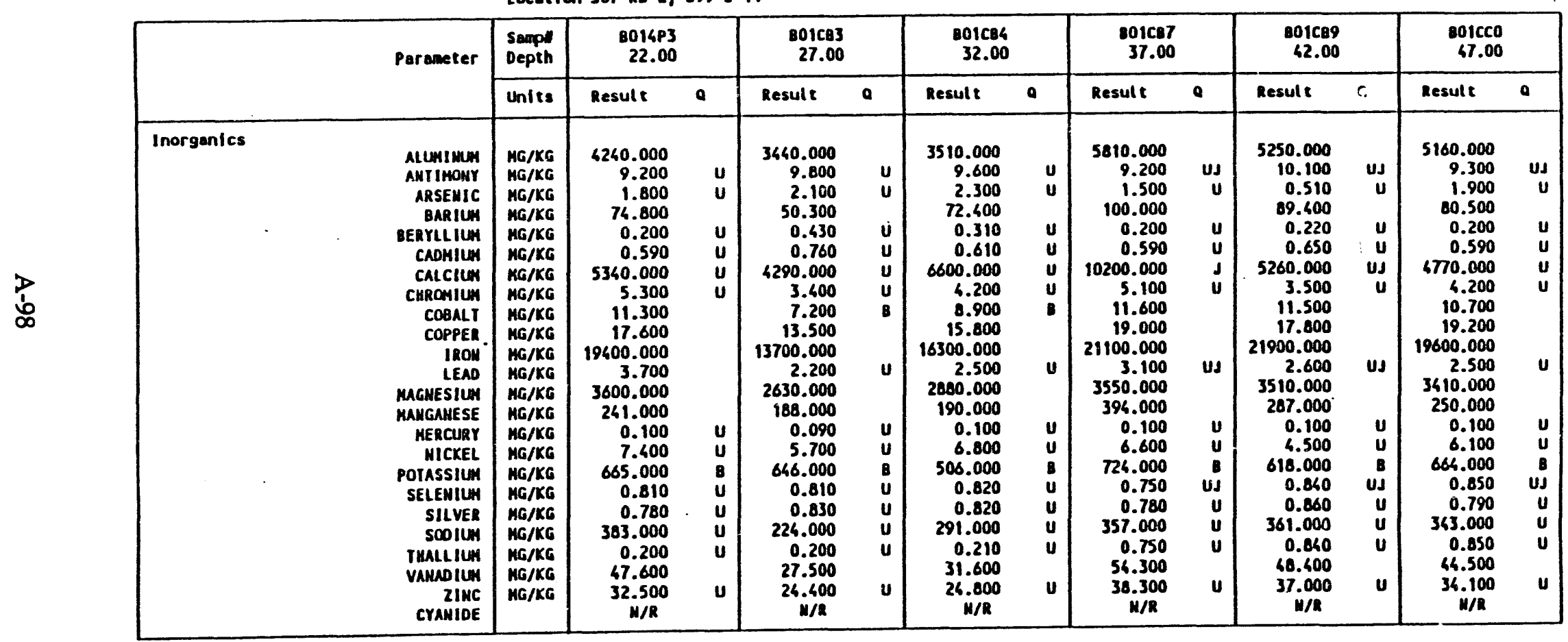


WHC-SD-EN-TI-279, Rev. 0

Location 307 RB-2, 399-3-16

\begin{tabular}{|c|c|c|c|c|c|c|}
\hline \multirow[t]{2}{*}{ - } & \multirow[t]{2}{*}{ Peraneter } & \multirow{2}{*}{$\begin{array}{l}\text { Semp: } \\
\text { Depth }\end{array}$} & \multicolumn{2}{|l|}{$\begin{array}{r}\text { B01C61 } \\
49.00\end{array}$} & \multicolumn{2}{|c|}{$\begin{array}{r}1016 c 2 \\
56.00\end{array}$} \\
\hline & & & Result & - & Resule & a \\
\hline Inorganics & 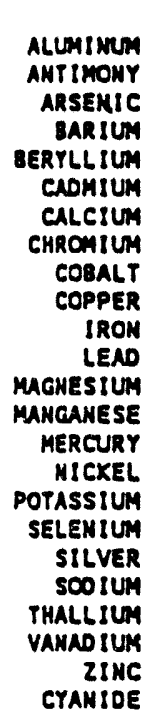 & $\begin{array}{l}M G / K G \\
M G / K G \\
M G / K G \\
M G / K G \\
M G / K G \\
M G / K G \\
M G / K G \\
M G / K G \\
M G / K G \\
M G / K G \\
M G / K G \\
M G / K G \\
M G / K G \\
M G / K G \\
M G / K G \\
M G / K G \\
\text { HG/KG } \\
M G / K G \\
M G / K G \\
M G / K G \\
M G / K G \\
M G / K G \\
M G / K G\end{array}$ & $\begin{array}{r}3210.000 \\
9.200 \\
1.000 \\
70.200 \\
0.200 \\
0.590 \\
3780.000 \\
4.300 \\
9.100 \\
16.100 \\
16600.000 \\
2.700 \\
2610.000 \\
191.000 \\
0.090 \\
4.700 \\
425.000 \\
0.780 \\
0.780 \\
214.000 \\
0.780 \\
39.600 \\
28.000 \\
1 / R\end{array}$ & $\begin{array}{c}\text { UJ } \\
U \\
U \\
U \\
U \\
U \\
B \\
U \\
U \\
U \\
U \\
u \\
U S \\
U \\
U \\
U \\
U\end{array}$ & $\begin{array}{r}3950.000 \\
10.200 \\
1.700 \\
81.800 \\
0.220 \\
0.650 \\
4850.000 \\
4.700 \\
10.000 \\
16.500 \\
21300.000 \\
2.700 \\
3120.000 \\
289.000 \\
0.090 \\
6.000 \\
506.000 \\
0.870 \\
0.860 \\
238.000 \\
0.870 \\
67.400 \\
33.700 \\
1 / 2\end{array}$ & $\begin{array}{r}U \\
U \\
B \\
U J \\
U \\
U \\
U \\
U\end{array}$ \\
\hline
\end{tabular}


WHC-SD-EN-TI-279, Rev. 0

This page left intentionally blank. 
Location 307 7-1, 399-3-15

\begin{tabular}{|c|c|c|c|c|c|c|c|c|c|c|c|c|c|c|}
\hline & \multirow[t]{2}{*}{ Par ameter } & \multirow{2}{*}{$\begin{array}{l}\text { Somp: } \\
\text { Depth }\end{array}$} & \multicolumn{2}{|c|}{$\begin{array}{r}801 \text { CF9 } \\
4.60\end{array}$} & \multicolumn{2}{|c|}{$\begin{array}{r}801 \text { CGO } \\
7.00\end{array}$} & \multicolumn{2}{|l|}{$\begin{array}{r}801 C 63 \\
9.00\end{array}$} & \multicolumn{2}{|c|}{$\begin{array}{l}\text { Bo1cGs } \\
11.40\end{array}$} & \multicolumn{2}{|l|}{$\begin{array}{r}801 c 05 \\
13.60\end{array}$} & \multicolumn{2}{|c|}{$\begin{array}{r}\text { Boicco } \\
15.00\end{array}$} \\
\hline & & & Result & 0 & Result & 0 & Result & a & Result & 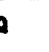 & Result & a & Result & 0 \\
\hline Inorganics & 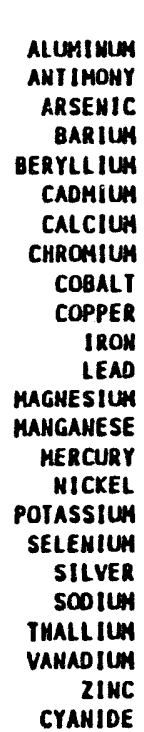 & $\begin{array}{l}M G / K G \\
M G / K G \\
H G / K G \\
H G / K G \\
M G / K G \\
M G / K G \\
M G / K G \\
M G / K G \\
M G / K G \\
H G / K G \\
M G / K G \\
M G / K G \\
M G / K G \\
M G / K G \\
H G / K G \\
M G / K G \\
H G / K G \\
M G / K G \\
M G / K G \\
H G / K G \\
M G / K G \\
H G / K G \\
M G / K G\end{array}$ & $\begin{array}{r}5730.000 \\
1.600 \\
1.500 \\
63.600 \\
0.200 \\
0.310 \\
4390.000 \\
19.200 \\
8.200 \\
20.000 \\
16500.000 \\
4.800 \\
3690.000 \\
271.000 \\
0.100 \\
9.600 \\
1040.000 \\
4.000 \\
0.400 \\
144.000 \\
0.400 \\
39.700 \\
37.300 \\
y / 8\end{array}$ & $\begin{array}{c}\text { UJ } \\
\mathbf{U} \\
J \\
\mathbf{U} \\
\mathbf{u} \\
\mathbf{U} \\
\mathbf{J} \\
\boldsymbol{\theta} \\
\mathbf{J} \\
\mathbf{J} \\
\mathbf{U} \\
\mathbf{U} \\
\mathbf{U} \\
\mathbf{U} \\
\mathbf{U} \\
\mathbf{U} \\
\mathbf{U} \\
\mathbf{U}\end{array}$ & $\begin{array}{r}8480.000 \\
1.600 \\
1.300 \\
75.600 \\
0.390 \\
0.290 \\
5540.000 \\
19.700 \\
9.100 \\
6.700 \\
18600.000 \\
6.000 \\
3850.000 \\
301.000 \\
0.100 \\
9.000 \\
1240.000 \\
0.800 \\
0.400 \\
165.000 \\
0.400 \\
46.000 \\
39.200 \\
W / R\end{array}$ & $\begin{array}{c}\text { UJ } \\
\mathbf{U} \\
\mathbf{J} \\
\mathbf{u} \\
\mathbf{u} \\
\mathbf{u} \\
\mathbf{J} \\
\mathbf{B} \\
\mathbf{U} \\
\mathbf{J}\end{array}$ & $\begin{array}{r}5300.000 \\
1.600 \\
2.100 \\
58.000 \\
0.200 \\
0.200 \\
5090.000 \\
19.500 \\
8.000 \\
10.100 \\
16300.000 \\
30.500 \\
3540.000 \\
251.000 \\
0.200 \\
9.400 \\
941.000 \\
3.900 \\
0.400 \\
156.000 \\
0.390 \\
40.100 \\
39.000 \\
W / R\end{array}$ & $\begin{array}{c}\mathbf{U J} \\
\mathbf{U} \\
\mathbf{J} \\
\mathbf{u} \\
\mathbf{u} \\
\mathbf{u} \\
\mathbf{J} \\
\mathbf{U} \\
\mathbf{U} \\
\mathbf{J}\end{array}$ & $\begin{array}{r}4910.000 \\
7.500 \\
1.800 \\
56.700 \\
0.290 \\
0.360 \\
4010.000 \\
8.100 \\
6.900 \\
14.900 \\
15000.000 \\
5.200 \\
3340.000 \\
243.000 \\
0.090 \\
8.200 \\
982.000 \\
0.780 \\
1.000 \\
120.000 \\
0.590 \\
35.200 \\
34.200 \\
11 / 8\end{array}$ & $\begin{array}{l}\mathbf{u} \\
\mathbf{u} \\
\mathbf{u} \\
\mathbf{u} \\
\mathbf{u} \\
\mathbf{u} \\
\mathbf{u} \\
\mathbf{u}\end{array}$ & $\begin{array}{r}5190.000 \\
6.000 \\
1.900 \\
67.600 \\
0.280 \\
0.700 \\
3390.000 \\
8.200 \\
6.900 \\
15.600 \\
15300.000 \\
5.000 \\
3680.000 \\
279.000 \\
0.090 \\
8.200 \\
1060.000 \\
0.020 \\
0.960 \\
107.000 \\
0.610 \\
35.100 \\
39.200 \\
W / R\end{array}$ & $\begin{array}{l}\mathbf{u} \\
\mathbf{u} \\
\mathbf{u} \\
\mathbf{u} \\
\mathbf{u} \\
\mathbf{u} \\
\mathbf{u}\end{array}$ & $\begin{array}{r}5320.000 \\
8.000 \\
2.800 \\
198.000 \\
0.290 \\
0.560 \\
4080.000 \\
9.800 \\
6.800 \\
62.300 \\
16000.000 \\
110.000 \\
3630.000 \\
306.000 \\
0.520 \\
10.200 \\
1070.000 \\
0.810 \\
5.500 \\
119.000 \\
0.610 \\
36.300 \\
98.200 \\
N / R\end{array}$ & $\begin{array}{l}u \\
u \\
u \\
u \\
u\end{array}$ \\
\hline
\end{tabular}


Location 307 T-1, 399-3-15

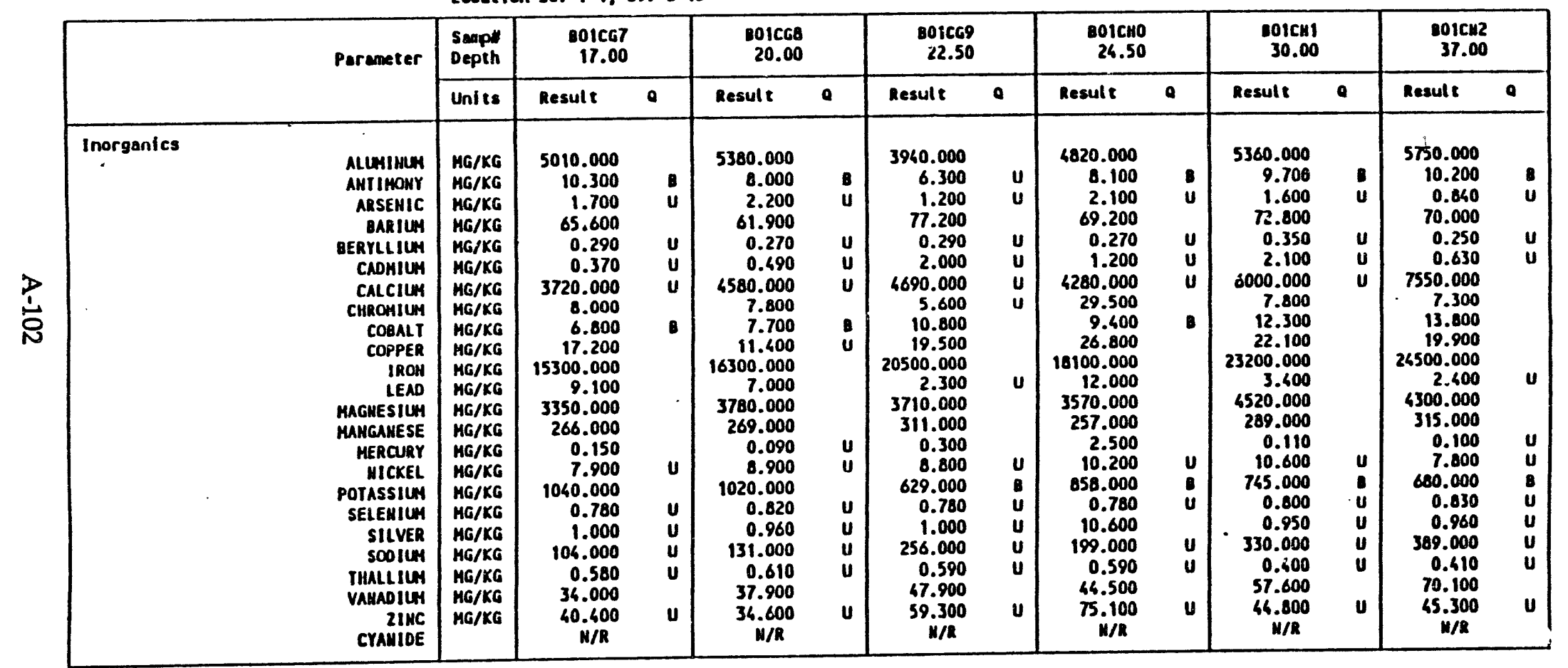


Location $307 \mathrm{r}-1,399-3-15$

\begin{tabular}{|c|c|c|c|c|c|c|c|c|c|c|c|c|}
\hline & \multirow[t]{2}{*}{ Parameter } & \multirow{2}{*}{$\begin{array}{l}\text { Sampl } \\
\text { Depth }\end{array}$} & \multicolumn{2}{|l|}{$\begin{array}{r}8018.10 \\
42.00\end{array}$} & \multicolumn{2}{|l|}{$\begin{array}{r}\text { Bo1RJ1 } \\
47.00\end{array}$} & \multicolumn{2}{|l|}{$\begin{array}{r}8018 J 2 \\
53.00\end{array}$} & \multicolumn{2}{|l|}{$\begin{array}{r}8018 . J 3 \\
58.00\end{array}$} & \multicolumn{2}{|c|}{$\begin{array}{r}8011 \mathrm{s6} \\
68.00\end{array}$} \\
\hline & & & Result & $a$ & Result & $a$ & Result & 0 & Result & $a$ & Result & a \\
\hline Inorganics & 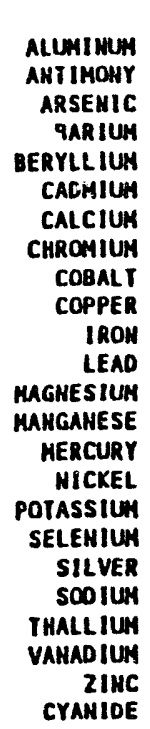 & $\begin{array}{l}M G / K G \\
M G / K G \\
M G / K G \\
M G / K G \\
M G / K G \\
M G / K G \\
M G / K G \\
M G / K G \\
M G / K G \\
M G / K G \\
M G / K G \\
M G / K G \\
M G / K G \\
M G / K G \\
M G / K G \\
M G / K G \\
M G / K G \\
M G / K G \\
\text { HG/KG } \\
M G / K G \\
M G / K G \\
M G / K G \\
M G / K G\end{array}$ & $\begin{array}{r}4880.000 \\
6.100 \\
1.600 \\
83.100 \\
0.260 \\
2.800 \\
6550.000 \\
274.000 \\
12.300 \\
22.000 \\
22400.000 \\
3.300 \\
3590.000 \\
325.000 \\
0.170 \\
146.000 \\
624.000 \\
0.820 \\
0.980 \\
250.000 \\
0.410 \\
50.800 \\
39.900 \\
W / R\end{array}$ & $\begin{array}{r}u \\
J \\
J \\
J \\
\mathbf{B} \\
\mathbf{U} J \\
\mathbf{U} \\
\mathbf{U} \\
\mathbf{U} \\
\mathbf{U}\end{array}$ & $\begin{array}{r}4400.000 \\
7.000 \\
1.300 \\
84.900 \\
0.260 \\
0.520 \\
478 j .000 \\
4.400 \\
10.900 \\
16.700 \\
20900.000 \\
2.600 \\
3310.000 \\
260.000 \\
0.160 \\
5.900 \\
541.000 \\
4.000 \\
0.980 \\
226.000 \\
0.400 \\
44.500 \\
37.200 \\
W / R\end{array}$ & $\begin{array}{c}\text { u } \\
J \\
d \\
\text { uJ } \\
\text { us } \\
u \\
u \\
u \\
\text { us } \\
\text { us }\end{array}$ & $\begin{array}{r}16800.000 \\
7.600 \\
1.500 \\
61.200 \\
0.990 \\
0.390 \\
11800.000 \\
12.400 \\
5.300 \\
16.700 \\
12800.000 \\
11.200 \\
6140.000 \\
258.000 \\
0.110 \\
7.900 \\
4860.000 \\
5.100 \\
1.200 \\
198.000 \\
0.510 \\
21.700 \\
31.100 \\
M / R\end{array}$ & $\begin{array}{c}\text { J } \\
\text { UJ } \\
\text { UJ } \\
u \\
u \\
\text { U } \\
\text { UJ }\end{array}$ & $\begin{array}{r}5130.000 \\
4.700 \\
2.200 \\
92.200 \\
0.650 \\
0.510 \\
5340.000 \\
5.200 \\
10.200 \\
19.300 \\
18700.000 \\
3.800 \\
3540.000 \\
393.000 \\
0.140 \\
6.800 \\
764.000 \\
4.400 \\
0.420 \\
209.000 \\
0.680 \\
42.200 \\
39.500 \\
\text { W/R }\end{array}$ & $\begin{array}{c}J \\
U \\
U \\
\text { UR } \\
\mathbf{U} \\
\mathbf{U} \\
\mathbf{U} \\
\mathbf{U}\end{array}$ & $\begin{array}{r}4530.000 \\
8.600 \\
2.300 \\
79.600 \\
0.220 \\
0.640 \\
4390.000 \\
5.100 \\
10.100 \\
21.000 \\
19100.000 \\
5.100 \\
3550.000 \\
264.000 \\
0.090 \\
7.500 \\
689.000 \\
0.850 \\
1.000 \\
244.000 \\
.0 .420 \\
34.800 \\
38.300 \\
1 / \pi\end{array}$ & $\begin{array}{l}u \\
u \\
u \\
u \\
u \\
u \\
u \\
u\end{array}$ \\
\hline
\end{tabular}


WHC-SD-EN-TI-279, Rev. 0

This page left intentionally blank. 
Location 307 T-2, 399-3-16

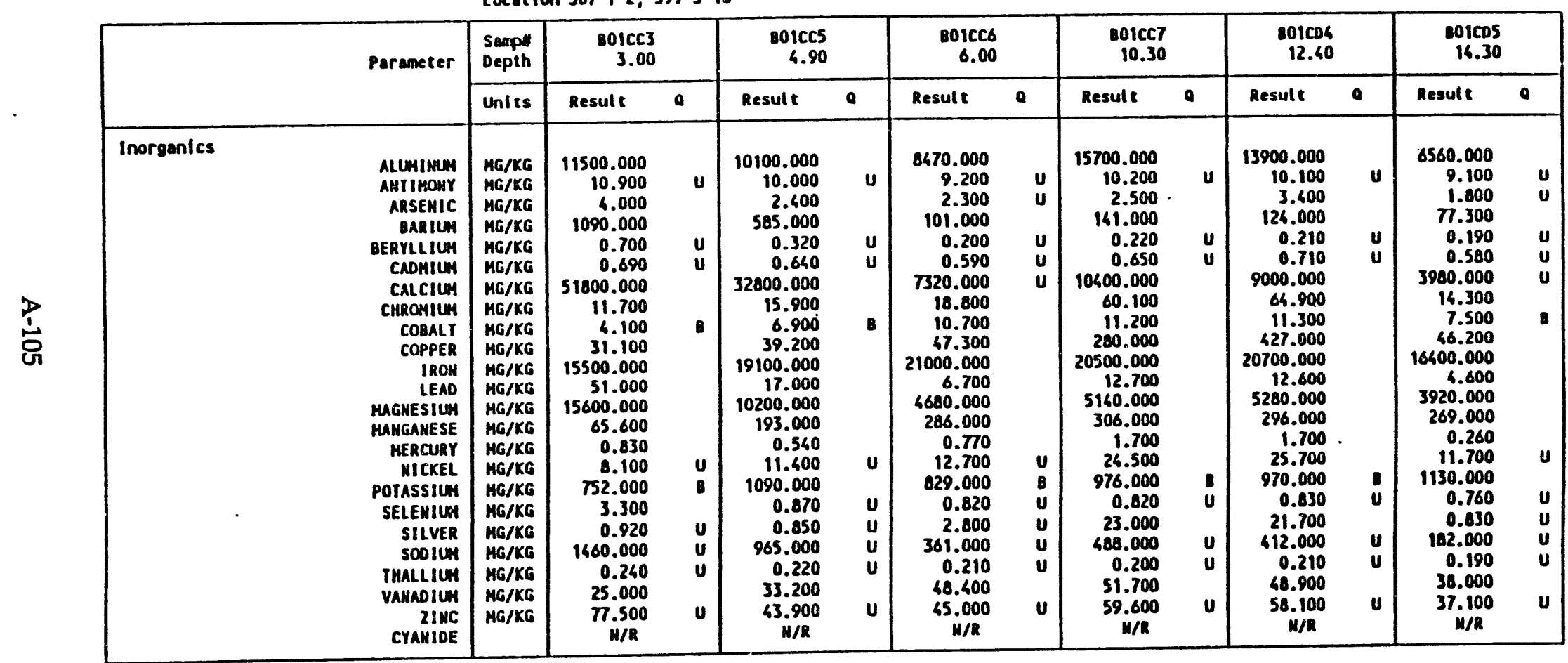


Locet I on 307 T-2, 399-3-16

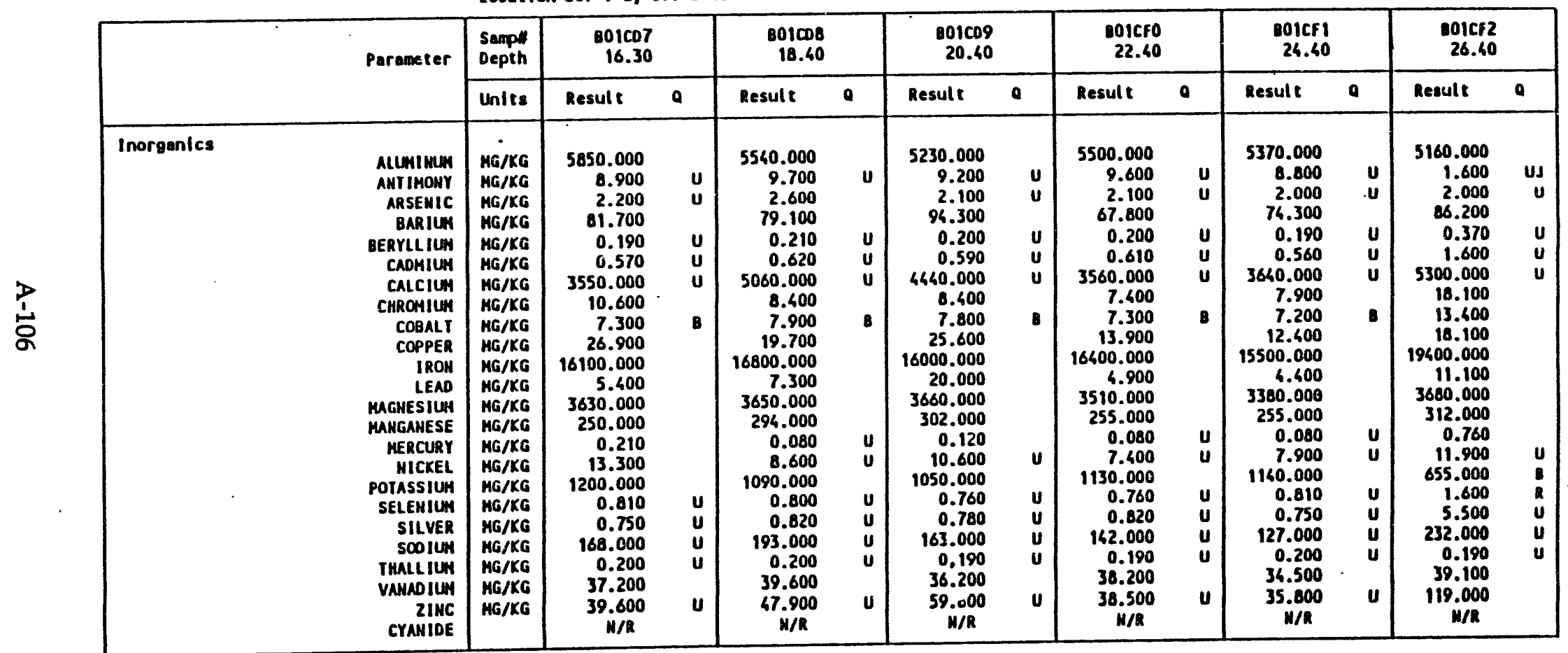


Location 307 1-2, 399-3-16

\begin{tabular}{|c|c|c|c|c|c|c|c|c|c|c|c|c|c|c|}
\hline & \multirow[t]{2}{*}{ Parameter } & \multirow{2}{*}{\begin{tabular}{l|} 
Sempl \\
Depth
\end{tabular}} & \multicolumn{2}{|l|}{$\begin{array}{r}\text { BOICF3 } \\
30.70\end{array}$} & \multicolumn{2}{|l|}{$\begin{array}{r}801 C F 4 \\
36.00\end{array}$} & \multicolumn{2}{|l|}{$\begin{array}{r}801 C F 5 \\
41.00 \\
\end{array}$} & \multicolumn{2}{|l|}{$\begin{array}{r}801 \text { Cf6 } 6 \\
47.00\end{array}$} & \multicolumn{2}{|l|}{$\begin{array}{r}001 c f 7 \\
58.00\end{array}$} & \multicolumn{2}{|l|}{$\begin{array}{r}\text { Boicrs } \\
60.00\end{array}$} \\
\hline & & & Result & 0 & Result & 0 & Result & 0 & Result & a & Result & - & Result & a \\
\hline Inorganics & 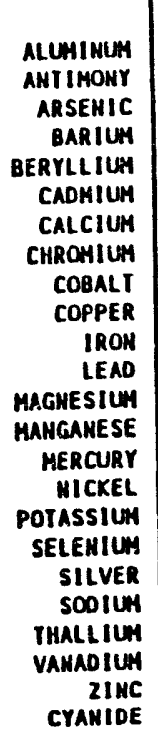 & $\begin{array}{l}H G / K G \\
H G / K G \\
H G / K G \\
H G / K G \\
H G / K G \\
H G / K G \\
H G / K G \\
M G / K G \\
H G / K G \\
H G / K G \\
H G / K G \\
H G / K G \\
H G / K G \\
M G / K G \\
H G / K G \\
H G / K G \\
H G / K G \\
H G / K G \\
M G / K G \\
M G / K G \\
M G / K G \\
H G / K G \\
M G / K G\end{array}$ & $\begin{array}{r}5310.000 \\
1.600 \\
1.400 \\
118.000 \\
0.200 \\
4.100 \\
7740.000 \\
7.900 \\
12.000 \\
15.900 \\
21800.000 \\
3.500 \\
3850.000 \\
308.000 \\
0.090 \\
8.000 \\
754.000 \\
0.820 \\
0.390 \\
310.000 \\
0.200 \\
58.800 \\
50.600 \\
\mathbf{W} / \mathrm{R}\end{array}$ & $\begin{array}{r}u \\
u \\
u \\
U R \\
u \\
u \\
u \\
u\end{array}$ & $\begin{array}{r}5910.000 \\
1.500 \\
1.400 \\
89.700 \\
0.780 \\
1.200 \\
8640.000 \\
12.900 \\
13.500 \\
22.100 \\
27800.000 \\
3.000 \\
4020.000 \\
330.000 \\
0.090 \\
10.200 \\
793.000 \\
0.760 \\
0.370 \\
507.000 \\
0.760 \\
68.600 \\
50.400 \\
\text { M/R }\end{array}$ & $\begin{array}{c}u J \\
u \\
u \\
u \\
J \\
\\
u \\
J \\
u \\
u \\
u J \\
u J \\
u J \\
u \\
J \\
u J\end{array}$ & $\begin{array}{r}6110.000 \\
1.600 \\
1.300 \\
99.900 \\
1.100 \\
0.200 \\
4380.000 \\
5.700 \\
12.400 \\
17.100 \\
22700.000 \\
3.500 \\
3460.000 \\
269.000 \\
0.110 \\
6.000 \\
686.000 \\
0.880 \\
0.410 \\
298.000 \\
0.880 \\
55.300 \\
43.000 \\
\mathbf{W} / \mathrm{R}\end{array}$ & $\begin{array}{r}\mathbf{J} \\
\mathbf{U} \\
\mathbf{U} \\
\mathbf{U} \\
\mathbf{U J} \\
\mathbf{U J} \\
\mathbf{U} \\
\mathbf{J} \\
\mathbf{U J}\end{array}$ & $\begin{array}{r}5620.000 \\
1.600 \\
1.200 \\
85.600 \\
1.200 \\
0.200 \\
6220.000 \\
7.800 \\
10.600 \\
16.100 \\
19800.000 \\
3.000 \\
3070.000 \\
255.000 \\
0.100 \\
7.600 \\
585.000 \\
0.750 \\
0.400 \\
313.000 \\
0.750 \\
51.100 \\
39.100 \\
M / R\end{array}$ & $\begin{array}{c}u \\
J \\
U \\
U \\
\text { UJ } \\
\text { UJ } \\
\mathbf{U J} \\
\mathbf{U} \\
\mathbf{J} \\
\mathbf{U J}\end{array}$ & $\begin{array}{r}4360.000 \\
1.500 \\
1.100 \\
83.000 \\
0.890 \\
0.410 \\
4660.000 \\
4.400 \\
9.600 \\
13.700 \\
20000.000 \\
2.300 \\
2930.000 \\
237.000 \\
0.090 \\
4.800 \\
601.000 \\
0.770 \\
0.370 \\
299.000 \\
0.770 \\
43.900 \\
36.100 \\
\mathbf{W} / \mathrm{R}\end{array}$ & $\begin{array}{r}u \\
J \\
u \\
u \\
u J \\
u J \\
u J \\
u \\
J \\
u J J\end{array}$ & $\begin{array}{r}4520.000 \\
1.600 \\
1.700 \\
77.500 \\
0.210 \\
0.210 \\
460.000 \\
5.600 \\
11.000 \\
20.100 \\
21500.000 \\
2.300 \\
3290.000 \\
232.000 \\
0.100 \\
6.300 \\
447.000 \\
0.030 \\
0.610 \\
393.000 \\
0.030 \\
56.700 \\
39.300 \\
M / R\end{array}$ & 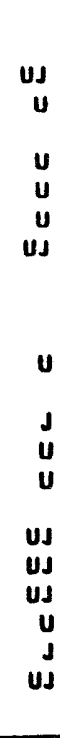 \\
\hline
\end{tabular}


WHC-SD-EN-TI-279, Rev. 0

This page left intentionally blank. 
Location 307 1-3, 399-3-17

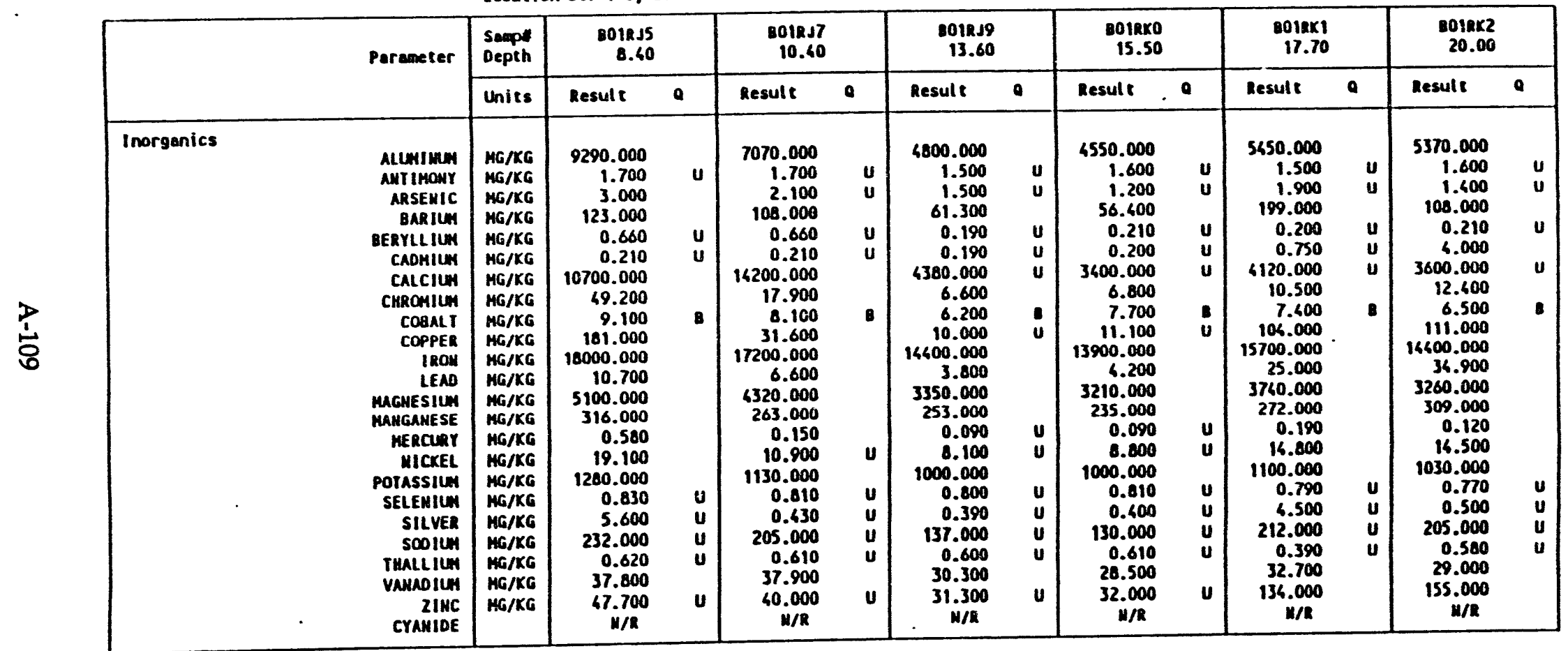




\begin{tabular}{|c|c|c|c|c|c|c|c|c|c|c|c|c|c|c|}
\hline & \multirow[t]{2}{*}{ Parameter } & \multirow{2}{*}{$\begin{array}{l}\text { Sespl } \\
\text { Depth }\end{array}$} & \multicolumn{2}{|l|}{$\begin{array}{r}\text { e01RX3 } \\
22.00\end{array}$} & \multicolumn{2}{|l|}{$\begin{array}{r}8018 \times 44 \\
24.00 \\
\end{array}$} & \multicolumn{2}{|l|}{$\begin{array}{r}\text { BO1RK5 } \\
27.30\end{array}$} & \multicolumn{2}{|l|}{$\begin{array}{r}8012 \times 6 \\
33.00\end{array}$} & \multicolumn{2}{|l|}{$\begin{array}{r}1018 \times 7 \\
38.00\end{array}$} & \multicolumn{2}{|l|}{$\begin{array}{r}8018 \times 9 \\
52.00\end{array}$} \\
\hline & & & Result & a & Result & - & Result & $a$ & Result & - & Result & a & Result & $\mathbf{a}$ \\
\hline Inorganics & 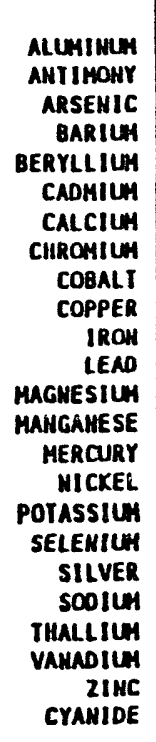 & 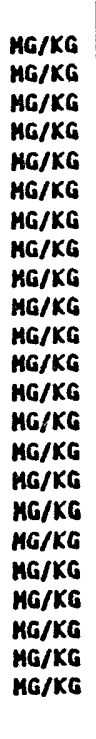 & $\begin{array}{r}5110.000 \\
1.600 \\
1.700 \\
73.800 \\
0.620 \\
0.200 \\
4660.000 \\
7.500 \\
9.600 \\
16.700 \\
10100.000 \\
5.100 \\
3560.000 \\
255.000 \\
0.090 \\
7.900 \\
1030.000 \\
0.850 \\
0.400 \\
274.000 \\
0.630 \\
40.000 \\
37.200 \\
\text { W/R }\end{array}$ & $\begin{array}{l}\mathbf{u} \\
\mathbf{u} \\
\boldsymbol{u} \\
\mathbf{u} \\
\mathbf{u} \\
\mathbf{u} \\
\mathbf{u}\end{array}$ & $\begin{array}{r}5890.000 \\
1.600 \\
1.100 \\
66.000 \\
0.610 \\
0.200 \\
4380.000 \\
19.200 \\
10.500 \\
17.900 \\
19600.000 \\
4.200 \\
3890.000 \\
272.000 \\
0.100 \\
14.600 \\
1050.000 \\
0.780 \\
0.390 \\
437.000 \\
0.590 \\
48.800 \\
45.700 \\
\text { N/R }\end{array}$ & $\begin{array}{l}u \\
u \\
u \\
u \\
u \\
u\end{array}$ & $\begin{array}{r}r 050.000 \\
1.600 \\
1.100 \\
83.100 \\
0.630 \\
1.100 \\
5040.000 \\
6.200 \\
11.700 \\
17.700 \\
22400.000 \\
2.800 \\
3720.000 \\
263.000 \\
0.100 \\
7.000 \\
604.000 \\
0.800 \\
0.410 \\
293.000 \\
0.600 \\
47.100 \\
39.100 \\
11 / R \\
\end{array}$ & $\begin{array}{l}\mathbf{U} \\
\mathbf{U} \\
\mathbf{B} \\
\mathbf{u} \\
\mathbf{u} \\
\mathbf{u} \\
\mathbf{U} \\
\mathbf{U}\end{array}$ & $\begin{array}{r}6330.000 \\
1.700 \\
1.000 \\
58.400 \\
0.640 \\
0.990 \\
6140.000 \\
5.200 \\
9.800 \\
21.800 \\
19200.000 \\
3.000 \\
3470.000 \\
250.000 \\
0.260 \\
7.200 \\
652.000 \\
0.790 \\
0.410 \\
277.000 \\
0.590 \\
61.900 \\
36.000 \\
W / R \\
\end{array}$ & $\begin{array}{l}u \\
u \\
u \\
u \\
u \\
u \\
v \\
u\end{array}$ & $\begin{array}{r}6520.000 \\
1.700 \\
0.960 \\
80.200 \\
0.650 \\
0.210 \\
6560.000 \\
6.200 \\
9.900 \\
16.100 \\
19500.000 \\
3.000 \\
3670.000 \\
256.000 \\
0.100 \\
7.000 \\
601.000 \\
0.750 \\
0.620 \\
326.000 \\
0.570 \\
42.900 \\
35.800 \\
\text { W/R }\end{array}$ & $\begin{array}{l}u \\
\\
u \\
u \\
u \\
u \\
u \\
u \\
u \\
u\end{array}$ & $\begin{array}{r}5340.000 \\
1.800 \\
1.900 \\
83.100 \\
0.220 \\
0.220 \\
4240.000 \\
4.600 \\
10.500 \\
17.100 \\
19600.000 \\
3.100 \\
3120.000 \\
693.000 \\
0.100 \\
6.900 \\
635.000 \\
0.690 \\
0.450 \\
201.000 \\
0.670 \\
50.100 \\
36.300 \\
1 / R\end{array}$ & $\begin{array}{l}u \\
u \\
u \\
B \\
u \\
u \\
u \\
u \\
u\end{array}$ \\
\hline
\end{tabular}


WHC-SD-EN-TI-279, Rev. 0

Location $3979-3,399-3 \cdot 17$

\begin{tabular}{|c|c|c|c|}
\hline \multirow[t]{2}{*}{ Paramater } & $\begin{array}{l}\text { Sampl } \\
\text { Depeth }\end{array}$ & \multicolumn{2}{|l|}{$\begin{array}{r}0018 \times 8 \\
65.00\end{array}$} \\
\hline & Units & Result & 0 \\
\hline 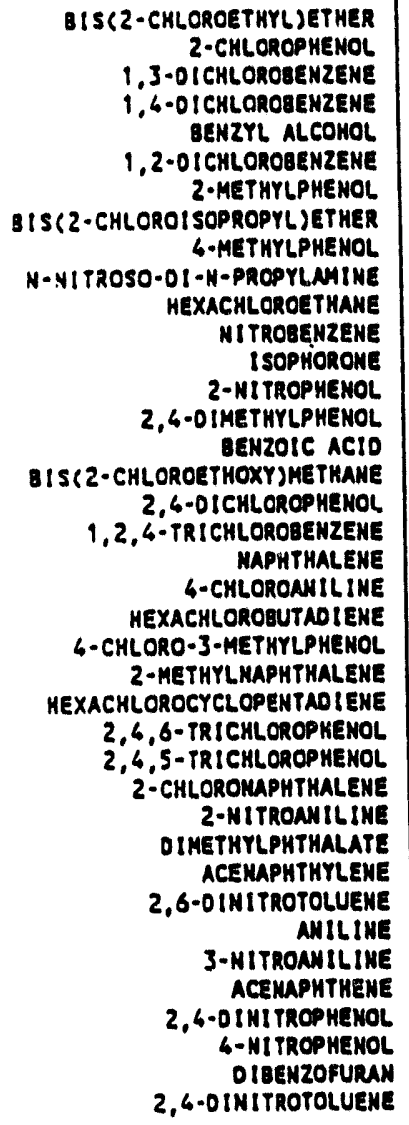 & . & 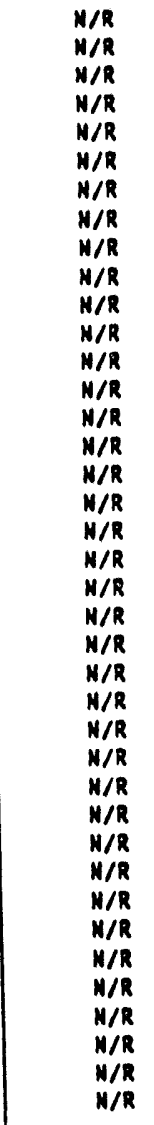 & \\
\hline
\end{tabular}


WHC-SD-EN-TI-279, Rev. 0

Location 307 r-3, 399-3-17

\begin{tabular}{|c|c|c|c|}
\hline \multirow[t]{2}{*}{ Paremeter } & \multirow{2}{*}{$\frac{\begin{array}{c}\text { Sampll. } \\
\text { Depth }\end{array}}{\text { Uni es }}$} & \multicolumn{2}{|c|}{$\begin{array}{r}801 R \times 8 \\
65.00\end{array}$} \\
\hline & & Resulte & 9 \\
\hline 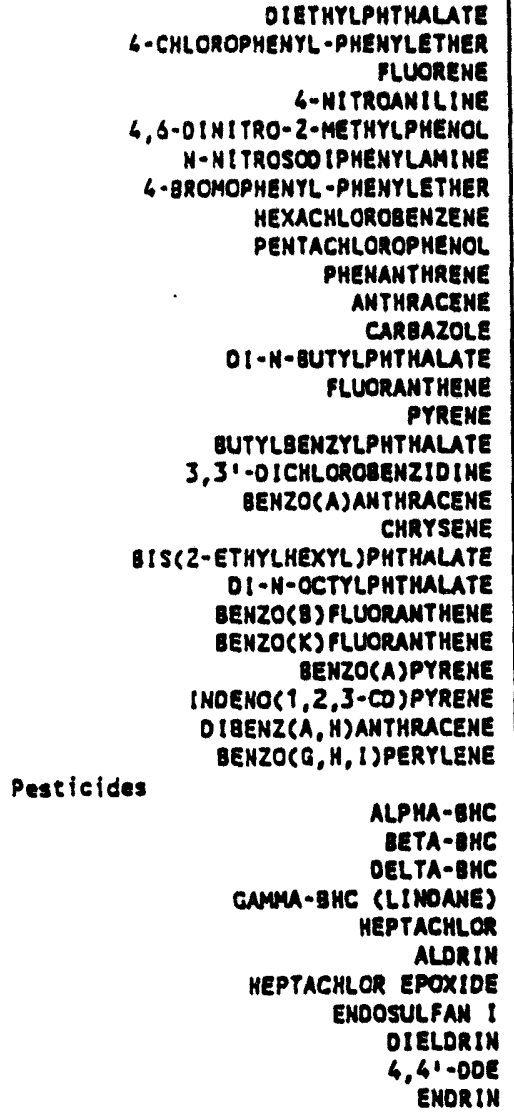 & $\begin{array}{l}U G / K G \\
U G / K G \\
U G / K G \\
U G / K G \\
U G / K G \\
U G / K G \\
U G / K G \\
U G / K G \\
U G / K G \\
U G / K G \\
U G / K G\end{array}$ & $\begin{array}{l}N / R \\
N / R \\
N / R \\
N / R \\
N / R \\
N / R \\
N / R \\
N / R \\
N / R \\
N / R \\
N / R \\
N / R \\
N / R \\
N / R \\
N / R \\
N / R \\
N / R \\
N / R \\
N / R \\
N / R \\
N / R \\
N / R \\
N / R \\
N / R \\
N / R \\
N / R \\
H / R \\
H / R \\
H / R \\
8.700 \\
8.700 \\
8.700 \\
8.700 \\
8.700 \\
8.700 \\
8.700 \\
8.700 \\
17.000 \\
17.000 \\
17.000\end{array}$ & $\begin{array}{l}u \\
u \\
u \\
u \\
u \\
u \\
u \\
u \\
u \\
u \\
u\end{array}$ \\
\hline
\end{tabular}


WHC-SD-EN-TI-279, Rev. 0

location 307 P-3, 399-3-17

\begin{tabular}{|c|c|c|c|c|}
\hline & \multirow[t]{2}{*}{ Parameter } & \multirow{2}{*}{$\frac{\text { Serpy }}{\text { Depeh }}$} & \multicolumn{2}{|l|}{$\begin{array}{r}\text { 101RK8 } \\
65.00\end{array}$} \\
\hline & & & Result & a \\
\hline${ }^{\circ}$ & 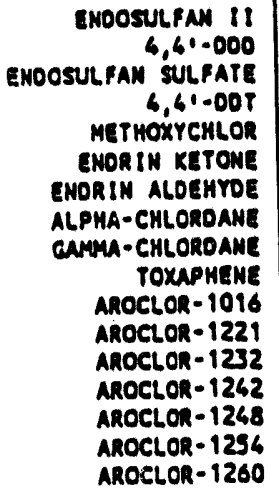 & $\begin{array}{l}\text { UG/KG } \\
\text { UG/KG } \\
\text { UG/KG } \\
\text { UG/KG } \\
\text { UG/KG } \\
\text { UG/KG } \\
\text { UG/KG } \\
\text { UG/KG } \\
\text { UG/KG } \\
\text { UG/KG } \\
\text { UG/KG } \\
\text { UG/KG } \\
\text { UG/KG } \\
\text { UG/KG } \\
\text { UG/KG } \\
\text { UG/KG }\end{array}$ & $\begin{array}{l}17.000 \\
17.000 \\
17.000 \\
17.000 \\
87.000 \\
17.000 \\
11 / 8 \\
87.000 \\
87.000 \\
170.000 \\
87.000 \\
87.000 \\
87.000 \\
87.000 \\
87.000 \\
170.000 \\
170.000\end{array}$ & $\begin{array}{l}u \\
u \\
u \\
u \\
u \\
u \\
u \\
u \\
u \\
u \\
u \\
u \\
u \\
u \\
u \\
u \\
u\end{array}$ \\
\hline
\end{tabular}


WHC-SD-EN-TT-279, Rev. 0

This page left intentionally blank. 
Location 307 RB-1, 399-3-13

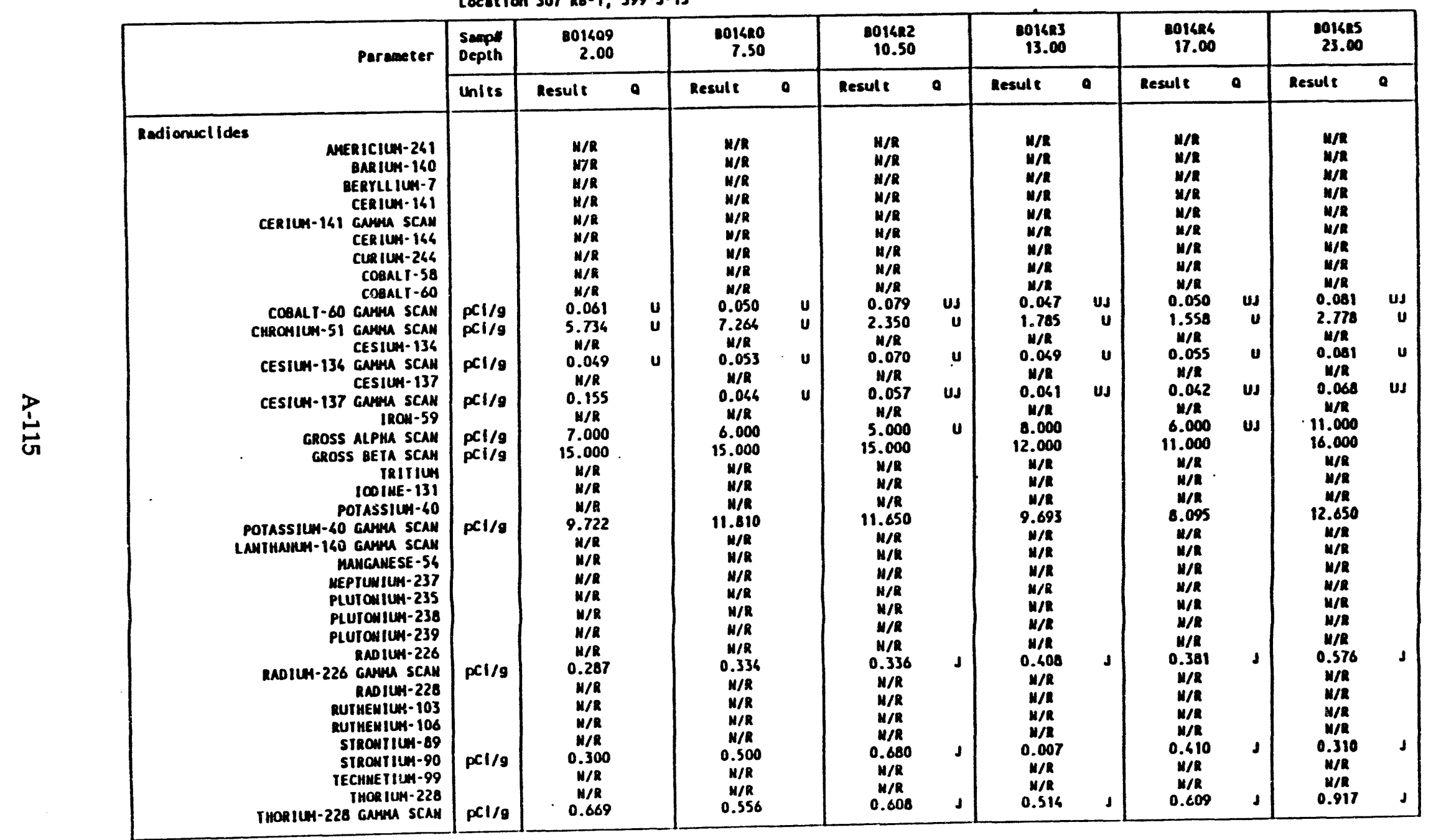


Locat Ion 307 R8-1, 399-3-13

\begin{tabular}{|c|c|c|c|c|c|c|c|c|c|c|c|c|c|c|}
\hline & \multirow[t]{2}{*}{ Parancter } & \multirow{2}{*}{\begin{tabular}{|l|} 
Semp: \\
Depth \\
Units \\
\end{tabular}} & \multicolumn{2}{|l|}{$\begin{array}{r}101409 \\
2.00\end{array}$} & \multicolumn{2}{|l|}{$\begin{array}{r}801680 \\
7.50\end{array}$} & \multicolumn{2}{|l|}{$\begin{array}{l}001422 \\
10.50\end{array}$} & \multicolumn{2}{|l|}{$\begin{array}{r}8014 a 3 \\
13.00\end{array}$} & \multicolumn{2}{|l|}{$\begin{array}{r}0014 R 6 \\
17.00\end{array}$} & \multicolumn{2}{|c|}{$\begin{array}{r}001685 \\
23.00\end{array}$} \\
\hline & & & Result & 0 & Result & $a$ & Result & $a$ & Result & 0 & Result & $a$ & Result & $a$ \\
\hline$\frac{5}{5}$ & 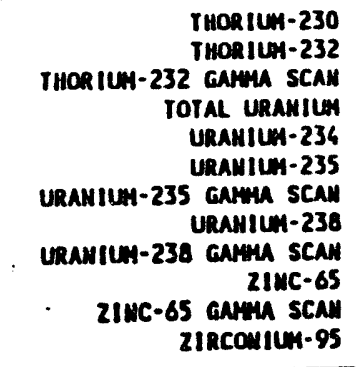 & $\begin{array}{l}\mathrm{pCl} / \mathrm{g} \\
\mathrm{pCl} / \mathrm{g} \\
\mathrm{pCl} / \mathrm{g} \\
\mathrm{pCl} / \mathrm{g} \\
\mathrm{pCl} / \mathrm{s}\end{array}$ & $\begin{array}{l}W / R \\
W / R \\
0.611 \\
W / R \\
0.800 \\
0.100 \\
W / R \\
0.600 \\
M / R \\
M / R \\
0.140 \\
M / R\end{array}$ & $\mathbf{u}$ & $\begin{array}{l}W / R \\
W / R \\
0.454 \\
W / R \\
6.100 \\
0.500 \\
W / R \\
5.400 \\
W / R \\
W / R \\
0.151 \\
W / R\end{array}$ & $\mathbf{u}$ & $\begin{array}{l}W / R \\
W / R \\
0.269 \\
W / R \\
0.700 \\
0.060 \\
W / R \\
0.600 \\
W / R \\
W / R \\
0.201 \\
W / R\end{array}$ & $\begin{array}{c}\text { Us } \\
\cdot \\
\text { J }\end{array}$ & $\begin{array}{c}M / R \\
M / R \\
0.608 \\
W / R \\
0.700 \\
0.070 \\
M / R \\
0.600 \\
W / R \\
W / R \\
0.119 \\
W / R\end{array}$ & J & $\begin{array}{l}M / R \\
M / R \\
0.637 \\
M / R \\
0.500 \\
0.090 \\
M / R \\
0.400 \\
M / R \\
M / R \\
0.162 \\
M / R\end{array}$ & $\begin{array}{l}J \\
J \\
J \\
J \\
U\end{array}$ & 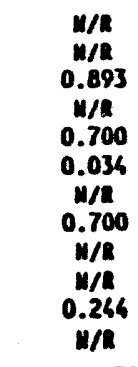 & $\mathbf{u}$ \\
\hline
\end{tabular}


Locat Ion 307 88-1, 399-3-13

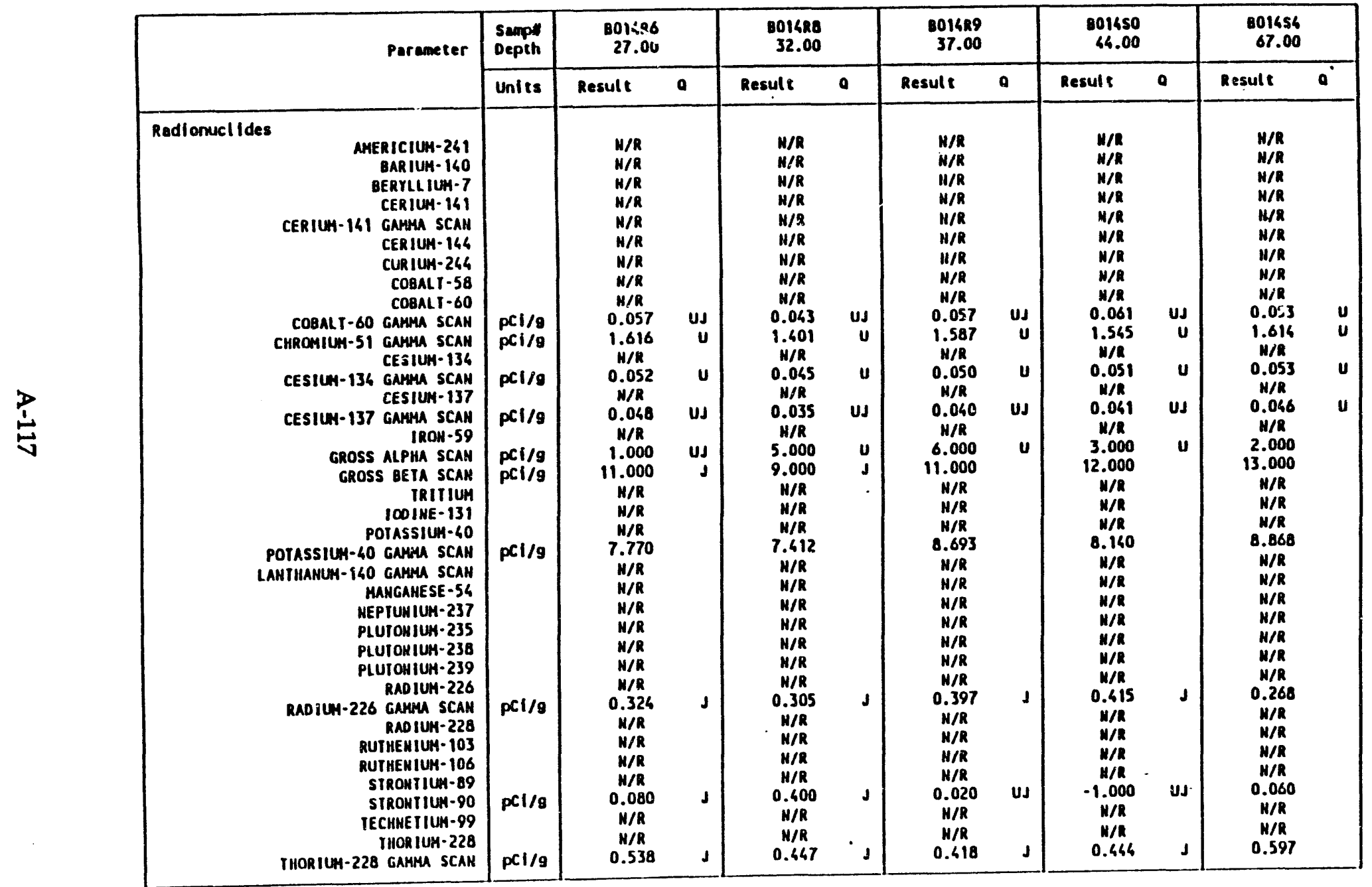


Location 307 RB-1, 399-3-13

\begin{tabular}{|c|c|c|c|c|c|c|c|c|c|c|c|c|}
\hline \multirow[b]{2}{*}{$\cdot$} & \multirow[t]{2}{*}{ Parameter } & \multirow{2}{*}{$\begin{array}{l}\text { Samp: } \\
\text { Depth }\end{array}$} & \multicolumn{2}{|l|}{$\begin{array}{r}8014 R 6 \\
27.00\end{array}$} & \multicolumn{2}{|l|}{$\begin{array}{r}\text { BO14R8 } \\
32.00\end{array}$} & \multicolumn{2}{|l|}{$\begin{array}{l}\text { BO14Ry } \\
37.00\end{array}$} & \multicolumn{2}{|l|}{$\begin{array}{r}801450 \\
44.00\end{array}$} & \multicolumn{2}{|c|}{$\begin{array}{r}801456 \\
67.00\end{array}$} \\
\hline & & & Result & a & Result & $a$ & Result & $a$ & Result & - & Result & $a$ \\
\hline . & 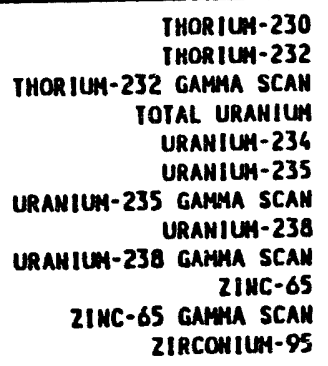 & $\begin{array}{l}\mathrm{pCl} / \mathrm{g} \\
\mathrm{pCl} / \mathrm{g} \\
\mathrm{pCI} / \mathrm{g} \\
\mathrm{pCl} / \mathrm{g} \\
\mathrm{pCl} / \mathrm{g}\end{array}$ & $\begin{array}{l}N / R \\
N / R \\
0.385 \\
N / R \\
0.600 \\
0.070 \\
N / R \\
0.800 \\
H / R \\
N / R \\
0.147 \\
N / R\end{array}$ & $\begin{array}{l}\mathbf{J} \\
\mathbf{J} \\
\mathbf{J} \\
\text { U }\end{array}$ & $\begin{array}{l}N / R \\
N / R \\
0.448 \\
N / R \\
0.500 \\
0.051 \\
N / R \\
0.600 \\
N / R \\
N / R \\
0.094 \\
N / R\end{array}$ & $\begin{array}{l}\mathbf{J} \\
\mathbf{J} \\
\mathbf{U}\end{array}$ & $\begin{array}{l}W / R \\
W / R \\
0.636 \\
M / R \\
0.500 \\
0.040 \\
M / R \\
0.600 \\
W / R \\
W / R \\
0.120 \\
H / R\end{array}$ & $\begin{array}{l}\mathbf{J} \\
\mathbf{J} \\
\mathbf{U}\end{array}$ & $\begin{array}{l}N / R \\
H / R \\
0.698 \\
N / R \\
0.600 \\
0.080 \\
W / R \\
0.500 \\
N / R \\
N / R \\
0.122 \\
H / R\end{array}$ & $\begin{array}{l}\mathbf{J} \\
\mathbf{J} \\
\mathbf{u}\end{array}$ & $\begin{array}{l}W / R \\
W / R \\
0.535 \\
M / R \\
1.200 \\
0.070 \\
W / R \\
1.000 \\
W / R \\
W / R \\
W / 137 \\
0.137 \\
H / R\end{array}$ & $u$ \\
\hline
\end{tabular}


WHC-SD-EN-TI-279, Rev. 0

This page left intentionally blank. 
Location 307 RB-2, 399-3-14

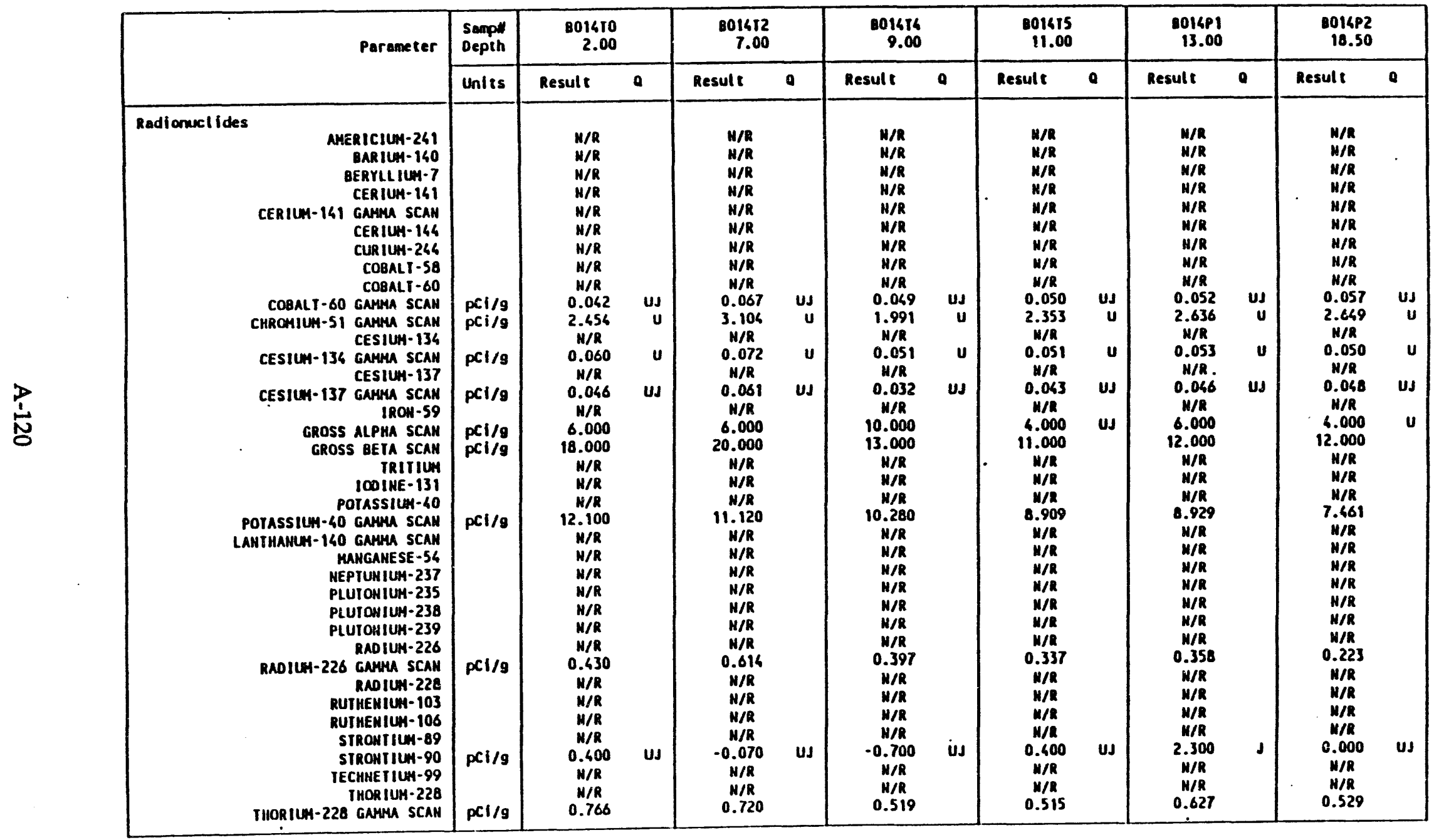




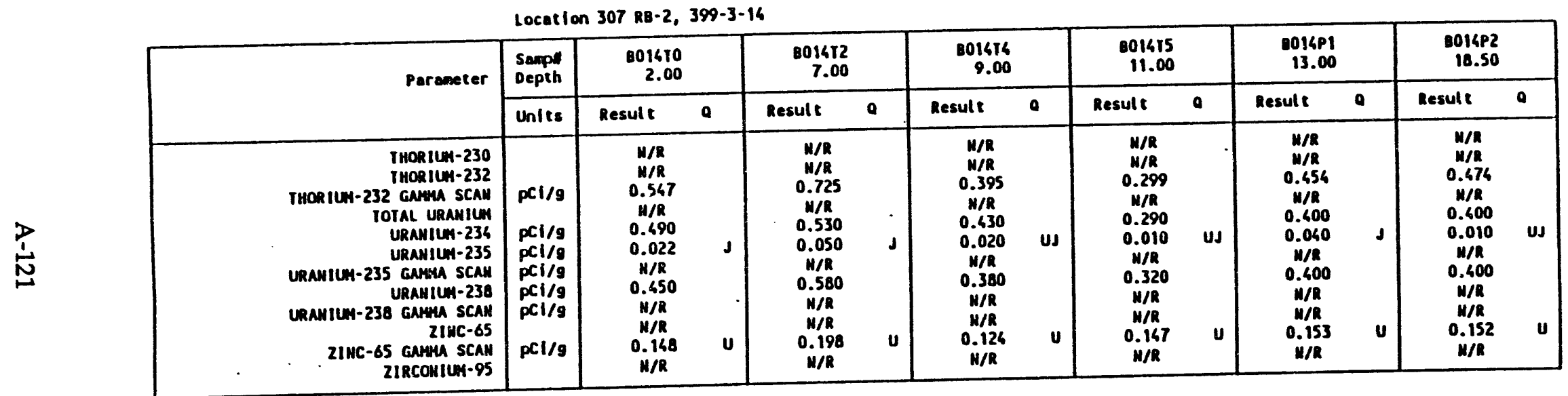




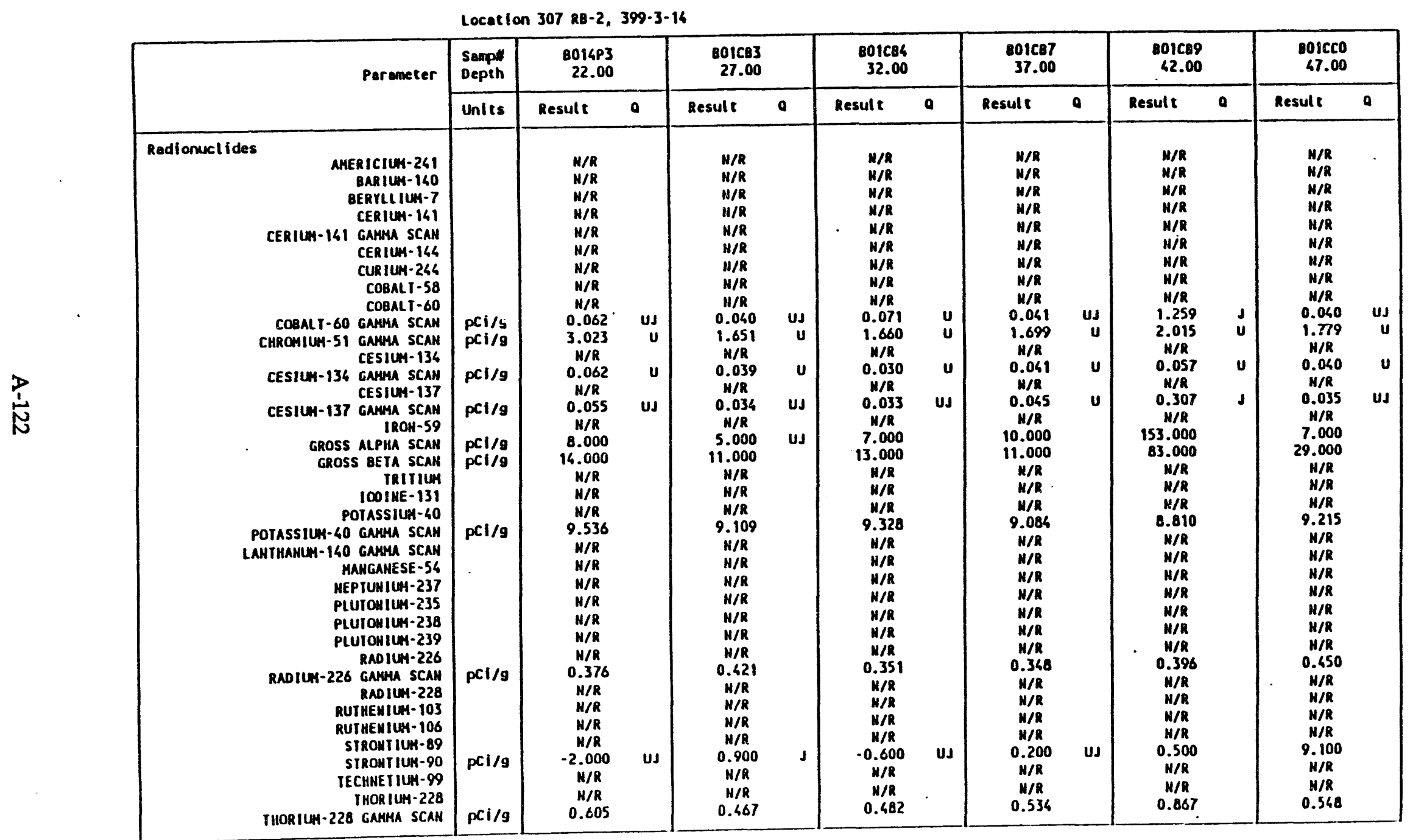


Location 307 RB-2, 399-3-14

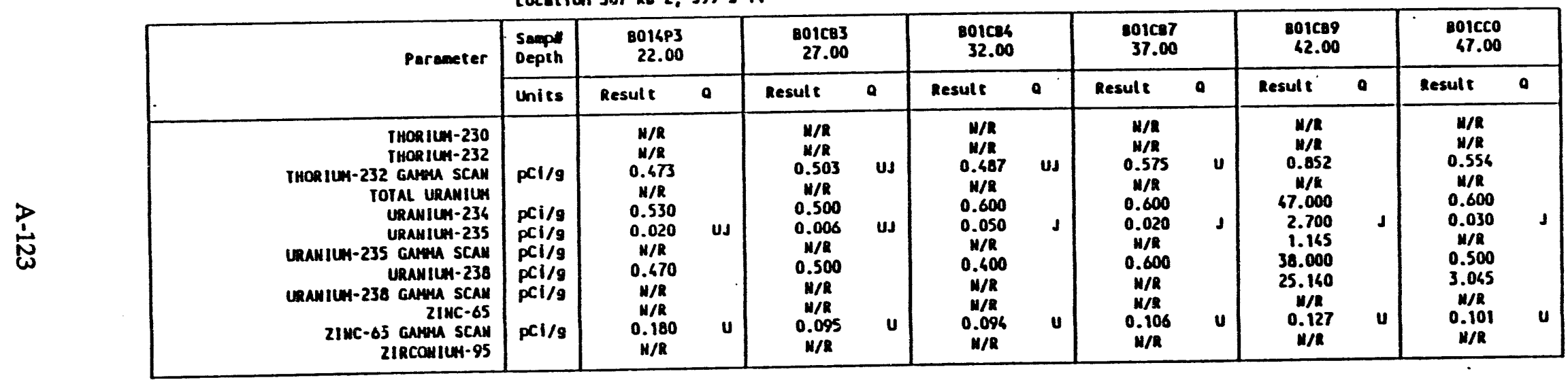


WHC-SD-EN-TI-279, Rev: 0

Locution 307 R8-2, 399-3-16

\begin{tabular}{|c|c|c|c|c|c|}
\hline \multirow[t]{2}{*}{ Parameter } & \multirow{2}{*}{$\begin{array}{l}\text { Sempll } \\
\text { Depen } \\
\text { Units }\end{array}$} & \multicolumn{2}{|c|}{$\begin{array}{r}8016 c 1 \\
69.00\end{array}$} & \multicolumn{2}{|l|}{$\begin{array}{r}8016 C 2 \\
56.00\end{array}$} \\
\hline & & Resule & $a$ & Result & 0 \\
\hline 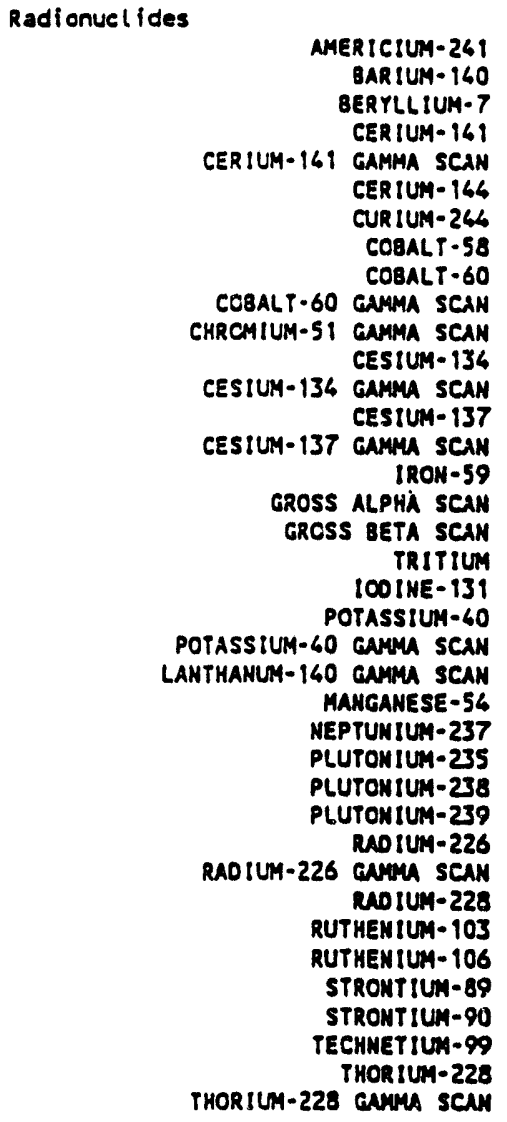 & $\begin{array}{l}\mathrm{pCl} / \mathrm{g} \\
\mathrm{pCl} / \mathrm{g} \\
\mathrm{pCi} / \mathrm{g} \\
\mathrm{pCl} / \mathrm{g} \\
\mathrm{pCl} / \mathrm{g} \\
\mathrm{pCl} / \mathrm{g} \\
\mathrm{pCl} / \mathrm{g}\end{array}$ & $\begin{array}{c}N / R \\
N / R \\
N / R \\
N / R \\
N / R \\
N / R \\
N / R \\
N / R \\
N / R \\
0.053 \\
2.608 \\
N / R \\
0.051 \\
N / R \\
0.050 \\
N / R \\
2.000 \\
19.000 \\
N / R \\
N / R \\
N / R \\
9.220 \\
N / R \\
N / R \\
N / R \\
N / R \\
N / R \\
N / R \\
N / R \\
O .362 \\
N / R \\
N / R \\
N / R \\
N / R \\
4.300 \\
N / R \\
N / R \\
0.565\end{array}$ & $\begin{array}{c}\text { UJ } \\
U \\
U \\
U J \\
U\end{array}$ & 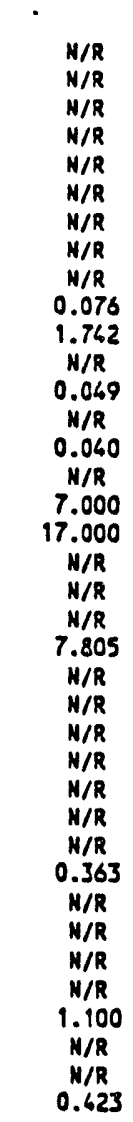 & $\begin{array}{c}\text { UJ } \\
U \\
U \\
\text { us }\end{array}$ \\
\hline
\end{tabular}


Location 307 RB-2, 399-3-16

\begin{tabular}{|c|c|c|c|c|c|}
\hline \multirow[t]{2}{*}{ Parameter } & \multirow{2}{*}{$\begin{array}{c}\text { Sampl } \\
\text { Depeh }\end{array}$} & \multicolumn{2}{|l|}{$\begin{array}{r}\text { Do1ce1 } \\
49.00\end{array}$} & \multicolumn{2}{|l|}{$\begin{array}{r}8010 C 2 \\
56.00\end{array}$} \\
\hline & & Resule & $a$ & Resule & 0 \\
\hline 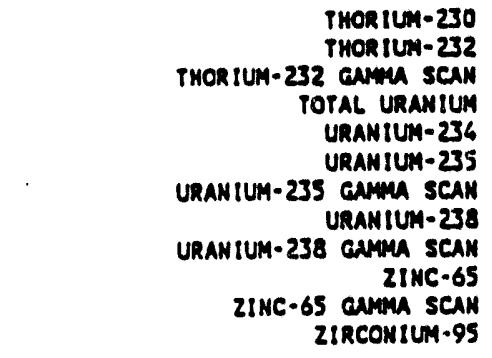 & $\begin{array}{l}p c i / g \\
p c i / g \\
p c i / g \\
p c i / g \\
p c i / g \\
p C i / g \\
p c i / g\end{array}$ & $\begin{array}{l}N / R \\
N / R \\
0.481 \\
N / R \\
0.500 \\
0.060 \\
N / R \\
0.500 \\
N / R \\
N / R \\
0.161 \\
N / R\end{array}$ & . & $\begin{array}{l}N / R \\
H / R \\
0.6 ! 1 \\
N / R \\
0.600 \\
0.020 \\
H / R \\
0.500 \\
N / R \\
N / R \\
0.125 \\
N / R\end{array}$ & $J$ \\
\hline
\end{tabular}


WHC-SD-EN-TI-279, Rev. 0

Locution 307 r-3, 399-3.17

\begin{tabular}{|c|c|c|c|c|}
\hline & \multirow[t]{2}{*}{ Parameter } & \multirow{2}{*}{$\begin{array}{l}\text { Sempel } \\
\text { Oepeh }\end{array}$} & \multicolumn{2}{|l|}{$\begin{array}{r}\text { So1nks } \\
65.00\end{array}$} \\
\hline & & & Resuls & a \\
\hline Inorgenies & 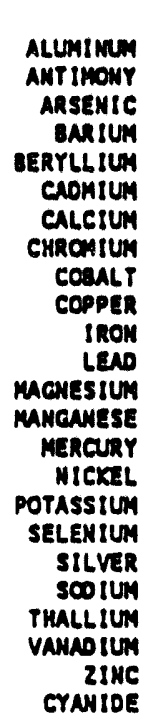 & $\begin{array}{l}\text { MG/KG } \\
\text { MG/KG } \\
\text { MG/KG } \\
\text { MG/KG } \\
\text { MO/KG } \\
\text { MG/KG } \\
\text { MG/KG } \\
\text { MG/KG } \\
\text { MG/KG } \\
\text { MG/KG } \\
\text { MG/KG } \\
\text { MG/KG } \\
\text { MG/KG } \\
\text { MG/KG } \\
\text { MG/KG } \\
\text { MG/KG } \\
\text { MG/KG } \\
\text { MG/KG } \\
\text { MO/KG } \\
\text { MG/KG } \\
\text { MO/KG } \\
\text { MG/KG } \\
\text { MO/KG }\end{array}$ & $\begin{array}{r}2930.000 \\
1.700 \\
1.600 \\
72.000 \\
0.210 \\
0.210 \\
3610.000 \\
3.300 \\
8.900 \\
19.800 \\
16500.000 \\
2.000 \\
2430.000 \\
202.000 \\
0.090 \\
4.000 \\
384.000 \\
0.840 \\
0.420 \\
263.000 \\
0.630 \\
45.900 \\
27.600 \\
M / R\end{array}$ & $\begin{array}{l}u \\
U \\
U \\
U \\
U \\
U \\
U \\
U\end{array}$ \\
\hline
\end{tabular}


WHC-SD-EN-TI-279, Rev. 0

Leention 307 r-3, 399-3-17

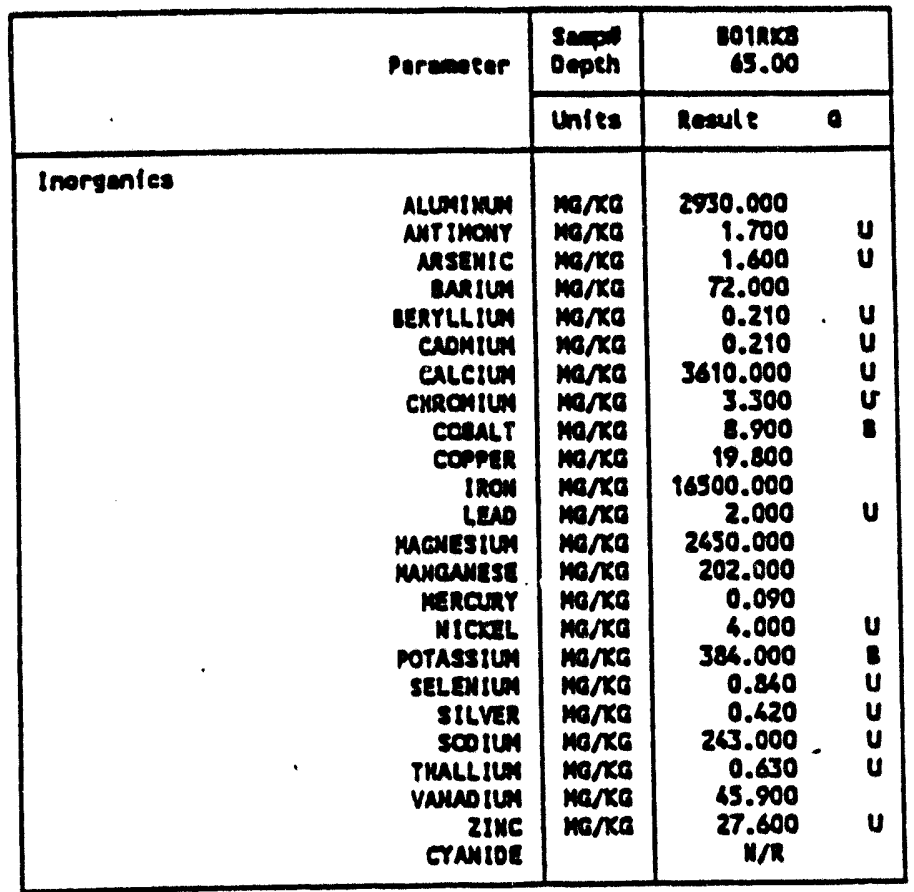


WHC-SD-EN-TI-279, Rev. 0

This page left intentionally blank. 
Locetlon 307 88-1, 399-3-13

\begin{tabular}{|c|c|c|c|c|c|c|c|c|c|c|c|c|c|c|}
\hline & \multirow[t]{2}{*}{ Parameter } & \multirow{2}{*}{$\begin{array}{l}\text { Scmp" } \\
\text { Depth } \\
\text { Unlts }\end{array}$} & \multicolumn{2}{|l|}{$\begin{array}{r}801609 \\
2.00 \\
\end{array}$} & \multicolumn{2}{|l|}{$\begin{array}{r}1014120 \\
7.50\end{array}$} & \multicolumn{2}{|l|}{$\begin{array}{r}2016 R 2 \\
10.50\end{array}$} & \multicolumn{2}{|l|}{$\begin{array}{r}101623 \\
13.00\end{array}$} & \multicolumn{2}{|l|}{$\begin{array}{r}001426 \\
17.00\end{array}$} & \multicolumn{2}{|l|}{$\begin{array}{r}0014.55 \\
23.00\end{array}$} \\
\hline & & & Result & - & Result & a & Result & a & Result & a & sesult & - & Result & - \\
\hline Chealistry & 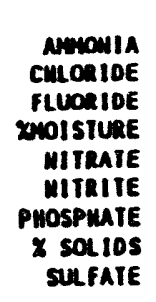 & $\begin{array}{l}\text { MG/KG } \\
\text { MG/KG } \\
X \\
\text { MG/KG } \\
\text { MG/KG } \\
\text { MG/KG }\end{array}$ & $\begin{array}{c}23.900 \\
W / R \\
0.500 \\
W / R \\
2.870 \\
0.500 \\
W / R \\
W / R \\
W / R \\
28.910\end{array}$ & $\begin{array}{l}\mathbf{u} \\
\mathbf{u} \\
\mathbf{u}\end{array}$ & $\begin{array}{c}27.900 \\
W / R \\
0.500 \\
W / R \\
3.640 \\
0.500 \\
W / R \\
W / R \\
W / R \\
29.750\end{array}$ & $\begin{array}{l}\text { u } \\
\text { u }\end{array}$ & $\begin{array}{c}15.960 \\
M / R \\
0.450 \\
w / R \\
w / 19 \\
2.190 \\
0.500 \\
w / R \\
W / R \\
26.490\end{array}$ & $\begin{array}{c}J \\
J \\
u \\
U J \\
J\end{array}$ & $\begin{array}{c}10.660 \\
w / 1 / \\
10.720 \\
5.510 \\
2.256 \\
2.2500 \\
0.5 / 1 \\
w / 2 \\
1 / 2 \\
35.130\end{array}$ & $\begin{array}{l}J \\
J \\
U \\
U \\
J\end{array}$ & $\begin{array}{c}9.310 \\
w / 1 \\
10.780 \\
5.100 \\
2.260 \\
0.500 \\
w / 1 \\
w / 1 \\
32.630 \\
32.630\end{array}$ & $\begin{array}{c}d \\
J \\
u \\
u \\
d\end{array}$ & $\begin{array}{c}10.660 \\
1 / 12 \\
15.590 \\
6.660 \\
9.230 \\
0.580 \\
w / 1 \\
w / 2 \\
1 / 1 \\
95.000\end{array}$ & $\begin{array}{l}\text { J } \\
\text { d }\end{array}$ \\
\hline
\end{tabular}


Locatlon 307 18-1, 399-3-13

\begin{tabular}{|c|c|c|c|c|c|c|c|c|c|c|c|c|}
\hline & \multirow[t]{2}{*}{ Perameter } & \multirow{2}{*}{$\begin{array}{l}\text { Seepen } \\
\text { Depth } \\
\text { Inlts }\end{array}$} & \multicolumn{2}{|l|}{$\begin{array}{r}001486 \\
27.00\end{array}$} & \multicolumn{2}{|l|}{$\begin{array}{r}801428 \\
32.00\end{array}$} & \multicolumn{2}{|l|}{$\begin{array}{r}3014149 \\
37.00\end{array}$} & \multicolumn{2}{|l|}{$\begin{array}{r}001650 \\
46.00\end{array}$} & \multicolumn{2}{|c|}{$\begin{array}{r}001456 \\
67.00\end{array}$} \\
\hline & & & Result & $a$ & Result & $a$ & Result & - & Result & 0 & Result & a \\
\hline Chealstry & $\begin{array}{c}\text { MUMONIA } \\
\text { CHLORIDE } \\
\text { FLUORIDE } \\
\text { XWOISTURE } \\
\text { MIIRAIE } \\
\text { MITRIIE } \\
\text { PHOSPHAIE } \\
\text { X SOLIOS } \\
\text { SULFATE }\end{array}$ & $\begin{array}{r}M G / K G \\
M G / K G \\
X \\
M G / K G \\
M G / K G \\
\\
M G / K G\end{array}$ & $\begin{array}{c}7.980 \\
W / R \\
10.800 \\
W / R \\
2.210 \\
0.500 \\
w / R \\
w / R \\
32.600\end{array}$ & $\begin{array}{l}u \\
u\end{array}$ & $\begin{array}{c}5.320 \\
w / 2 \\
10.790 \\
w / R \\
2.300 \\
0.500 \\
w / 2 \\
w / 2 \\
29.050\end{array}$ & $\begin{array}{l}u \\
u\end{array}$ & $\begin{array}{c}5.000 \\
W / R \\
0.770 \\
W / R \\
2.100 \\
0.500 \\
W / R \\
W / R \\
31.500\end{array}$ & u & $\begin{array}{c}5.000 \\
w / R \\
11.200 \\
w / R \\
2.300 \\
0.500 \\
w / R \\
w / R \\
32.000\end{array}$ & $\begin{array}{l}\mathbf{u} \\
\mathbf{u}\end{array}$ & $\begin{array}{c}m / 1 \\
w / 2 \\
1.960 \\
1.96 \\
1 / 10 \\
1.700 \\
0.500 \\
w / 10 \\
w / 2 \\
90.500\end{array}$ & $\begin{array}{l}u \\
u\end{array}$ \\
\hline
\end{tabular}




\begin{tabular}{|c|c|c|c|c|c|c|c|c|c|c|c|c|c|c|}
\hline & \multirow[t]{2}{*}{ Paraneter } & \multirow{2}{*}{$\begin{array}{l}\text { Soepp } \\
\text { Depth }\end{array}$} & \multicolumn{2}{|l|}{$\begin{array}{r}801410 \\
2.00\end{array}$} & \multicolumn{2}{|l|}{$\begin{array}{r}801412 \\
7.00\end{array}$} & \multicolumn{2}{|l|}{$\begin{array}{r}0014.14 \\
9.00\end{array}$} & \multicolumn{2}{|l|}{$\begin{array}{r}801415 \\
11.00\end{array}$} & \multicolumn{2}{|l|}{$\begin{array}{r}\text { SO14P1 } \\
13.00\end{array}$} & \multicolumn{2}{|l|}{$\begin{array}{r}0014 P 2 \\
10.50 \\
\end{array}$} \\
\hline & & & Result & 9 & Result & 0 & Result & a & Result & a & Result & 0 & Result & 0 \\
\hline Chemistry & $\begin{array}{r}\text { ANHONIA } \\
\text { CHLORIDE } \\
\text { FLURIDE } \\
\text { XHOISIURE } \\
\text { MITRAIE } \\
\text { HIRTIIE } \\
\text { PHOSPHAIE } \\
\text { X SOLIOS } \\
\text { SULFATE }\end{array}$ & $\begin{array}{l}\mathrm{MG} / \mathrm{KG} \\
\mathrm{HG} / \mathrm{KG} \\
\mathrm{HG} / \mathrm{KG} \\
\mathrm{MG} / \mathrm{KG} \\
\mathrm{MG} / \mathrm{KG}\end{array}$ & $\begin{array}{c}16.000 \\
\text { W/R } \\
9.900 \\
M / R \\
2.8 \\
2.300 \\
0.500 \\
W / R \\
W / R \\
\text { W/R } \\
30.400\end{array}$ & $\begin{array}{c}J \\
J \\
\text { US } \\
\text { UJ }\end{array}$ & $\begin{array}{c}14.600 \\
M / R \\
1.900 \\
H / R \\
W .90 \\
2.900 \\
0.500 \\
W / R \\
H / R \\
30.000\end{array}$ & $\begin{array}{c}J \\
J \\
\text { UJ } \\
\text { US }\end{array}$ & $\begin{array}{c}0.000 \\
W / R \\
2.100 \\
W / R \\
2.100 \\
0.500 \\
W / R \\
W / R \\
34.300\end{array}$ & $\begin{array}{l}\text { J } \\
\text { J } \\
\text { UJ } \\
\text { US }\end{array}$ & $\begin{array}{c}0.000 \\
W / R \\
2.100 \\
W / R \\
2.100 \\
0.500 \\
W / R \\
W / R \\
35.300\end{array}$ & $\begin{array}{c}J \\
J \\
\text { US } \\
\text { US }\end{array}$ & $\begin{array}{c}5.000 \\
W / R \\
6.500 \\
M / R \\
2.800 \\
0.500 \\
M / R \\
W / R \\
34.200\end{array}$ & $\begin{array}{l}u \\
u\end{array}$ & $\begin{array}{c}5.000 \\
W / R \\
6.000 \\
M / R \\
1 . R \\
1.700 \\
0.500 \\
W / R \\
W / R \\
36.400 \\
36.400 \\
\end{array}$ & u \\
\hline
\end{tabular}


Location 307 RB-2, 399-3-16

\begin{tabular}{|c|c|c|c|c|c|c|c|c|c|c|c|c|c|c|}
\hline & \multirow[t]{2}{*}{ Parameter } & \multirow{2}{*}{\begin{tabular}{|l} 
Semp: \\
Depth
\end{tabular}} & \multicolumn{2}{|l|}{$\begin{array}{r}8014 P 3 \\
22.00\end{array}$} & \multicolumn{2}{|l|}{$\begin{array}{r}801 \mathrm{res}^{2} \\
27.00\end{array}$} & \multicolumn{2}{|l|}{$\begin{array}{r}801 C 84 \\
32.00\end{array}$} & \multicolumn{2}{|l|}{$\begin{array}{r}010107 \\
37.00\end{array}$} & \multicolumn{2}{|l|}{$\begin{array}{r}801 c 89 \\
62.00\end{array}$} & \multicolumn{2}{|c|}{$\begin{array}{r}801 C c 0 \\
47.00\end{array}$} \\
\hline & & & Result & $\mathbf{a}$ & Result & $a$ & Rtsult & a & Result & $a$ & Result & a & Result & 0 \\
\hline Chealstry & $\begin{array}{r}\text { AHMOHIA } \\
\text { CHLORIDE } \\
\text { FLURIDE } \\
\text { XHOISIURE } \\
\text { MITRAIE } \\
\text { HITRIIE } \\
\text { PHOSPHAIE } \\
\text { X SOLIDS } \\
\text { SULFATE }\end{array}$ & $\begin{array}{l}\text { MG/KG } \\
\text { MG/KG } \\
\text { MG/KG } \\
\text { HG/KG } \\
\\
\text { HG/KG }\end{array}$ & $\begin{array}{c}5.300 \\
H / R \\
7.500 \\
W / R \\
2.600 \\
0.500 \\
M / R \\
H / R \\
38.000\end{array}$ & $\begin{array}{l}\mathbf{u} \\
\mathbf{u}\end{array}$ & $\begin{array}{c}5.320 \\
W / R \\
0.650 \\
W / R \\
W / R \\
1.890 \\
0.500 \\
W / R \\
H / R \\
34.190\end{array}$ & $\begin{array}{l}\mathbf{u} \\
\mathbf{u}\end{array}$ & $\begin{array}{c}5.320 \\
W / R \\
10.330 \\
W / R \\
2.380 \\
0.500 \\
W / R \\
H / R \\
N 9.310\end{array}$ & $\begin{array}{l}u \\
u\end{array}$ & $\begin{array}{c}2.200 \\
H / R \\
2.600 \\
W / R \\
2.500 \\
0.500 \\
W / R \\
H / R \\
33.700\end{array}$ & $\begin{array}{l}\text { UJ } \\
\text { J } \\
\text { UJ } \\
\text { UJ } \\
\text { J }\end{array}$ & $\begin{array}{c}2.000 \\
W / R \\
1.200 \\
W / R \\
M / 200 \\
3.200 \\
0.500 \\
W / R \\
W / 2 \\
36.900\end{array}$ & $\begin{array}{l}\text { Us } \\
J \\
\text { Us } \\
\text { UJ } \\
\end{array}$ & $\begin{array}{c}1.800 \\
W / R \\
9.400 \\
W / R \\
2.000 \\
0.500 \\
W / R \\
W / R \\
30.500\end{array}$ & $\begin{array}{l}\text { US } \\
\text { J } \\
\text { Us }\end{array}$ \\
\hline
\end{tabular}


WHC-SD-EN-TI-279, Rev. 0

Lecetion 307 R8-2, 399-3-16

\begin{tabular}{|c|c|c|c|c|c|c|}
\hline & \multirow[t]{2}{*}{ Paremeter } & \multirow{2}{*}{$\begin{array}{l}\text { Sempil } \\
\text { Depeh }\end{array}$} & \multicolumn{2}{|c|}{$\begin{array}{r}\text { Bo1ect } \\
69.00\end{array}$} & \multicolumn{2}{|c|}{$\begin{array}{r}8016 c 2 \\
56.00\end{array}$} \\
\hline & & & Result & a & Resule & 0 \\
\hline Chemisery & 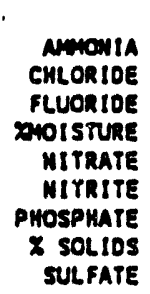 & $\begin{array}{l}\text { MG/KG } \\
\text { MG/KG } \\
\text { MG/KG } \\
\text { HG/KG } \\
\text { MG/KG }\end{array}$ & $\begin{array}{c}1.900 \\
N / R \\
8.900 \\
N / R \\
1.500 \\
0.500 \\
N / R \\
N / R \\
31.100\end{array}$ & $\begin{array}{l}\text { US } \\
\text { J } \\
\text { Ud } \\
\text { US }\end{array}$ & $\begin{array}{c}2.300 \\
N / R \\
1.000 \\
N / R \\
1.700 \\
0.500 \\
N / R \\
N / R \\
31.600\end{array}$ & $\begin{array}{l}\text { US } \\
\text { J } \\
\text { Ud } \\
\text { UJ }\end{array}$ \\
\hline
\end{tabular}




\begin{tabular}{|c|c|c|c|c|c|c|c|c|c|c|c|c|c|c|}
\hline & \multirow[t]{2}{*}{ Parameter } & \multirow{2}{*}{\begin{tabular}{|l|} 
Sampl \\
Depth
\end{tabular}} & \multicolumn{2}{|l|}{$\begin{array}{r}8016 F 9 \\
4.60\end{array}$} & \multicolumn{2}{|l|}{$\begin{array}{r}801 C 60 \\
7.00\end{array}$} & \multicolumn{2}{|l|}{$\begin{array}{r}801 C G 1 \\
9.00\end{array}$} & \multicolumn{2}{|l|}{$\begin{array}{l}001 c 66 \\
11.40\end{array}$} & \multicolumn{2}{|l|}{$\begin{array}{r}\text { Bo1c65 } \\
13.60\end{array}$} & \multicolumn{2}{|l|}{$\begin{array}{r}.001 \text { CG6 } \\
15.00\end{array}$} \\
\hline & & & Result & a & Result & a & Result & a & Result & a & Result & 0 & Result & 0 \\
\hline Chemistry & 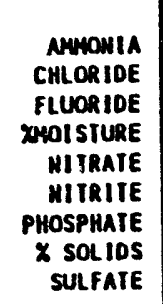 & $\begin{array}{l}\text { MG/KG } \\
\text { MG/KG } \\
\text { MG/KG } \\
\text { HG/KG } \\
\text { HG/KG }\end{array}$ & $\begin{array}{c}0.500 \\
M / R \\
W / .00 \\
W / R \\
W / R \\
6.100 \\
1.000 \\
W / R \\
W / R \\
34.000\end{array}$ & $\begin{array}{c}\text { UJ } \\
\text { UR } \\
\text { UJ }\end{array}$ & $\begin{array}{c}0.500 \\
W / R \\
1.000 \\
W / R \\
5.700 \\
1.000 \\
W / R \\
W / R \\
43.000\end{array}$ & $\begin{array}{c}\text { US } \\
\text { UR } \\
\text { UJ }\end{array}$ & $\begin{array}{c}1.200 \\
H / R \\
2.600 \\
W / R \\
10.700 \\
1.300 \\
H / R \\
W / R \\
25 / 0 \\
25.000\end{array}$ & $\begin{array}{l}u \\
u\end{array}$ & $\begin{array}{c}1.100 \\
\mu / R \\
1.000 \\
1 / / R \\
5.700 \\
1.000 \\
W / R \\
W / R \\
63.000\end{array}$ & $\begin{array}{l}u \\
u\end{array}$ & $\begin{array}{c}0.900 \\
W / R \\
1.000 \\
W / R \\
5 . R \\
5.900 \\
1.000 \\
W / R \\
W / R \\
36.000\end{array}$ & $\begin{array}{c}\mathbf{u} \\
\mathbf{u} \\
\mathbf{u}\end{array}$ & $\begin{array}{c}0.800 \\
\mu / R \\
1.000 \\
W / R \\
0.000 \\
1.000 \\
W / R \\
H / R \\
59.000\end{array}$ & $\begin{array}{l}\text { u } \\
\text { u }\end{array}$ \\
\hline
\end{tabular}




\begin{tabular}{|c|c|c|c|c|c|c|c|c|c|c|c|c|c|c|}
\hline & \multirow[t]{2}{*}{ Parameter } & \multirow{2}{*}{$\begin{array}{l}\text { Samp" } \\
\text { Depth } \\
\text { Units }\end{array}$} & \multicolumn{2}{|l|}{$\begin{array}{r}\text { 801CG7 } \\
17.00\end{array}$} & \multicolumn{2}{|l|}{$\begin{array}{r}\text { BO1CG8 } \\
20.00\end{array}$} & \multicolumn{2}{|l|}{$\begin{array}{r}801069 \\
22.50 \\
\end{array}$} & \multicolumn{2}{|l|}{$\begin{array}{r}\text { BO1CHO } \\
26.50\end{array}$} & \multicolumn{2}{|l|}{$\begin{array}{r}01 C 11 \\
30.00\end{array}$} & \multicolumn{2}{|l|}{$\begin{array}{r}201 \mathrm{CH2} \\
37.00\end{array}$} \\
\hline & & & Result & 0 & Result & $a$ & Result & 0 & Result & 0 & Result & 0 & Result & $a$ \\
\hline Chenlstry & $\begin{array}{c}\text { APHOMIA } \\
\text { CHLORIOE } \\
\text { FLURIDE } \\
\text { XHOISTURE } \\
\text { HITRAIE } \\
\text { HIRIIE } \\
\text { PHOSPHAIE } \\
\text { X SOL IDS } \\
\text {. SULFAIE }\end{array}$ & $\begin{array}{l}\text { MG/KG } \\
\text { MG/KG } \\
\text { HG/KG } \\
\text { HG/KG } \\
\text { MG/KG }\end{array}$ & $\begin{array}{c}0.900 \\
M / R \\
M .000 \\
1 . / R \\
S .700 \\
5.7000 \\
1.000 \\
N / R \\
M / R \\
37.000\end{array}$ & $\begin{array}{l}\text { u } \\
\text { u }\end{array}$ & $\begin{array}{c}0.700 \\
W / R \\
1.000 \\
W / R \\
6.500 \\
1.000 \\
W / R \\
W / R \\
36.000\end{array}$ & $\begin{array}{l}u \\
u\end{array}$ & $\begin{array}{c}0.800 \\
W / R \\
1.000 \\
W / R \\
6.400 \\
1.000 \\
W / R \\
W / R \\
37.000\end{array}$ & $\begin{array}{l}u \\
u\end{array}$ & $\begin{array}{c}0.800 \\
W / R \\
1.000 \\
W / R \\
7.800 \\
1.000 \\
W / R \\
W / R \\
39.000\end{array}$ & u & $\begin{array}{c}1.200 \\
W / R \\
1.000 \\
W / 8 \\
5.600 \\
1.000 \\
W / R \\
W / R \\
36.000\end{array}$ & $\begin{array}{l}u \\
u\end{array}$ & 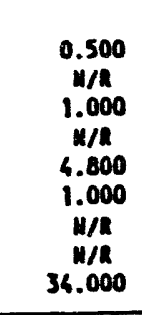 & $\begin{array}{l}u \\
u\end{array}$ \\
\hline
\end{tabular}




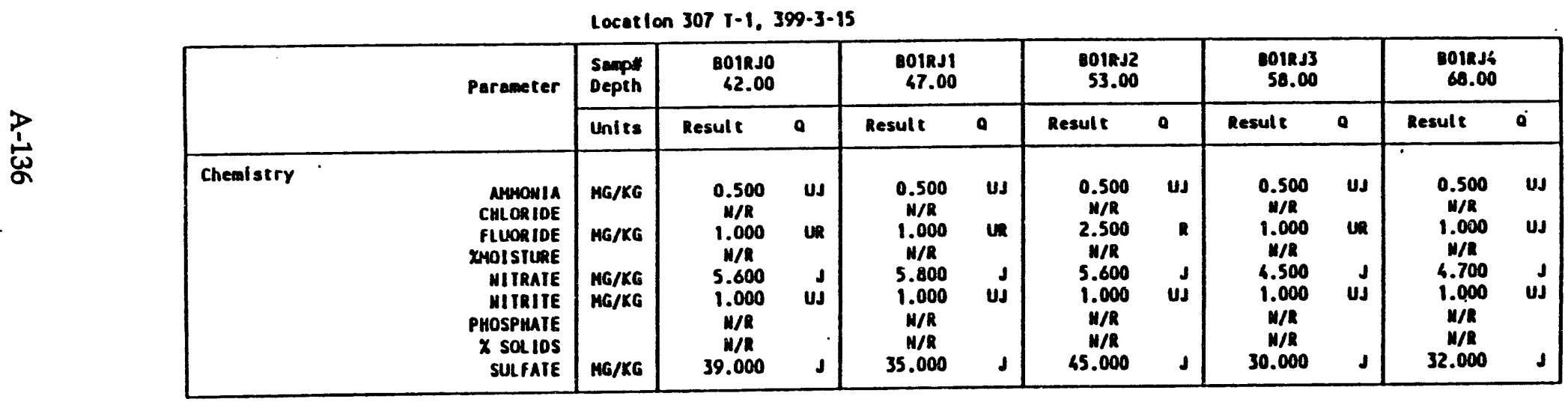




\begin{tabular}{|c|c|c|c|c|c|c|c|c|c|c|c|c|c|c|}
\hline & \multirow[t]{2}{*}{ Paremeter } & \multirow{2}{*}{\begin{tabular}{l|} 
Samp: \\
Depth \\
Units \\
\end{tabular}} & \multicolumn{2}{|l|}{$\begin{array}{r}801 \mathrm{CCJ}^{2} \\
3.00 \\
\end{array}$} & \multicolumn{2}{|l|}{$\begin{array}{r}801 c c 5 \\
4.90\end{array}$} & \multicolumn{2}{|l|}{$\begin{array}{r}8010 c 6 \\
6.00\end{array}$} & \multicolumn{2}{|l|}{$\begin{array}{l}801 \mathrm{cc7} \\
10.30\end{array}$} & \multicolumn{2}{|l|}{$\begin{array}{r}101006 \\
12.40\end{array}$} & \multicolumn{2}{|c|}{$\begin{array}{r}010105 \\
14.30\end{array}$} \\
\hline & & & Result & 0 & Result & - & Result & a & Result & a & Result & - & Result & a \\
\hline Chemistry & 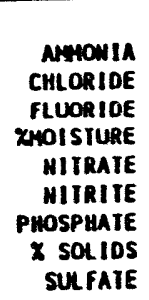 & $\begin{array}{l}\text { MG/KG } \\
\text { HG/KG } \\
M G / K G \\
M G / K G \\
\text { HG/KG }\end{array}$ & $\begin{array}{c}11.000 \\
H / R \\
7.870 \\
7 / R \\
37 / R \\
37.100 \\
0.500 \\
H / R \\
W / R \\
2150.000\end{array}$ & u & $\begin{array}{c}7.000 \\
W / R \\
3.950 \\
W / R \\
16.600 \\
0.500 \\
W / R \\
W / R \\
492.600\end{array}$ & $\begin{array}{l}J \\
J \\
J \\
J\end{array}$ & $\begin{array}{c}4.500 \\
1 \% / 8 \\
16.370 \\
w / R \\
10.300 \\
0.500 \\
W / 8 \\
W / 8 \\
70.400\end{array}$ & $\begin{array}{l}J \\
d \\
\text { us } \\
J\end{array}$ & $\begin{array}{c}10.200 \\
M / R \\
S .800 \\
M / R \\
46.400 \\
0.500 \\
W / R \\
M / R \\
47.000\end{array}$ & u & $\begin{array}{c}17.800 \\
M / R \\
0.100 \\
M / R \\
37.900 \\
0.500 \\
W / R \\
W / R \\
66.000\end{array}$ & $u$ & 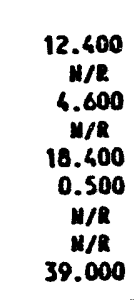 & u \\
\hline
\end{tabular}




\begin{tabular}{|c|c|c|c|c|c|c|c|c|c|c|c|c|c|c|}
\hline & \multirow[t]{2}{*}{ Parameter } & \multirow{2}{*}{$\begin{array}{l}\text { Sempt } \\
\text { Depth } \\
\text { Units }\end{array}$} & \multicolumn{2}{|l|}{$\begin{array}{r}801 C 07 \\
16.30\end{array}$} & \multicolumn{2}{|l|}{$\begin{array}{r}01 c 08 \\
18.60\end{array}$} & \multicolumn{2}{|l|}{$\begin{array}{r}0101009 \\
20.40 \\
\end{array}$} & \multicolumn{2}{|l|}{$\begin{array}{r}\text { BO1CFo } \\
22.60 \\
\end{array}$} & \multicolumn{2}{|l|}{$\begin{array}{r}\text { S01CFI } \\
26.40\end{array}$} & \multicolumn{2}{|c|}{$\begin{array}{r}\text { BO1CF2 } \\
26.40\end{array}$} \\
\hline & & & Result & 0 & Result & a & Result & $a$ & Result & - & Result & a & Result & 0 \\
\hline Chesalstry & 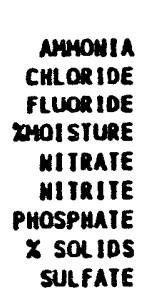 & $\begin{array}{l}\mathrm{MG} / \mathrm{KG} \\
\mathrm{HG/KG} \\
\mathrm{MG/KG} \\
\mathrm{MG} / \mathrm{KG} \\
\mathrm{MG} / \mathrm{KG}\end{array}$ & $\begin{array}{c}12.200 \\
W / 8 \\
3.300 \\
W / R \\
12.800 \\
1.000 \\
W / R \\
W / R \\
32.000\end{array}$ & v & $\begin{array}{c}9.500 \\
W / R \\
2.600 \\
W / R \\
10.300 \\
1.000 \\
W / R \\
W / R \\
32.000\end{array}$ & u & $\begin{array}{c}10.500 \\
M / R \\
2.700 \\
W / R \\
10.800 \\
1.000 \\
W / R \\
W / R \\
33.000\end{array}$ & u & $\begin{array}{c}9.400 \\
W / R \\
2.500 \\
W / 8 \\
10.900 \\
1.500 \\
W / R \\
W / R \\
33.000\end{array}$ & $\mathbf{u}$ & $\begin{array}{c}11.000 \\
W / R \\
2.400 \\
w / R \\
9.600 \\
0.500 \\
w / R \\
W / R \\
31.000\end{array}$ & u & $\begin{array}{c}22.000 \\
W / R \\
3.200 \\
W / R \\
W / .50 \\
12.000 \\
1.000 \\
W / R \\
W / R \\
60.000\end{array}$ & u \\
\hline
\end{tabular}


Location 307 7-2, 399-3-16

\begin{tabular}{|c|c|c|c|c|c|c|c|c|c|c|c|c|c|c|}
\hline & \multirow[t]{2}{*}{ Parameter } & \multirow{2}{*}{$\frac{\text { Sampl }}{\text { Depth }}$} & \multicolumn{2}{|c|}{$\begin{array}{r}\text { BOICF3 } \\
30.70\end{array}$} & \multicolumn{2}{|l|}{$\begin{array}{r}\text { BOICF6 } \\
36.00\end{array}$} & \multicolumn{2}{|l|}{$\begin{array}{r}801 \text { CF5 } \\
61.00\end{array}$} & \multicolumn{2}{|l|}{$\begin{array}{r}\text { s01Cf6 } \\
47.00\end{array}$} & \multicolumn{2}{|l|}{$\begin{array}{r}\text { cotcrf7 } \\
58.00\end{array}$} & \multicolumn{2}{|l|}{$\begin{array}{r}\text { So1cfo } \\
60.00\end{array}$} \\
\hline & & & Result & a & Result & a & Result & a & Result & $a$ & Result & $a$ & Resul $t$ & 0 \\
\hline Chealstry & $\begin{array}{r}\text { ANMONIA } \\
\text { CHHORIDE } \\
\text { FLURIDE } \\
\text { XMOISIURE } \\
\text { MIIRAIE } \\
\text { MIIRIIE } \\
\text { PHOSPHAIE } \\
\text { X SOLIDS } \\
\text { SULFAIE }\end{array}$ & $\begin{array}{l}\text { MG/KG } \\
\text { HG/KG } \\
\text { MG/KG } \\
\text { HG/KG } \\
\text { MG/KG }\end{array}$ & $\begin{array}{c}3.800 \\
H / R \\
3.200 \\
N / R \\
9.700 \\
1.000 \\
H / R \\
N / R \\
55.000\end{array}$ & $\mathbf{u}$ & $\begin{array}{c}2.100 \\
W / R \\
4.500 \\
H / R \\
H / 200 \\
8.2000 \\
1.000 \\
H / R \\
H / R \\
77.000\end{array}$ & J & $\begin{array}{c}2.000 \\
W / R \\
4.500 \\
W / R \\
W / 200 \\
6.500 \\
1.000 \\
W / R \\
W / R \\
59.000\end{array}$ & $\begin{array}{l}\text { J } \\
\text { U J } \\
J\end{array}$ & $\begin{array}{c}2.200 \\
W / R \\
6.000 \\
W / R \\
5.200 \\
1.000 \\
W / R \\
11 / R \\
37.000\end{array}$ & $\begin{array}{l}\text { J } \\
\text { us }\end{array}$ & $\begin{array}{c}1.000 \\
M / R \\
4.600 \\
M / R \\
1 \% / R \\
5.500 \\
1.000 \\
H / R \\
H / R \\
37.000\end{array}$ & d & $\begin{array}{c}2.400 \\
1 / 1 \\
1.000 \\
w / 1 / 2 \\
4.700 \\
1.000 \\
w / 2 \\
1 / 2 \\
32.000\end{array}$ & $\begin{array}{l}\text { US } \\
\text { US }\end{array}$ \\
\hline
\end{tabular}


Locetion 307 1-3, 399-3-17

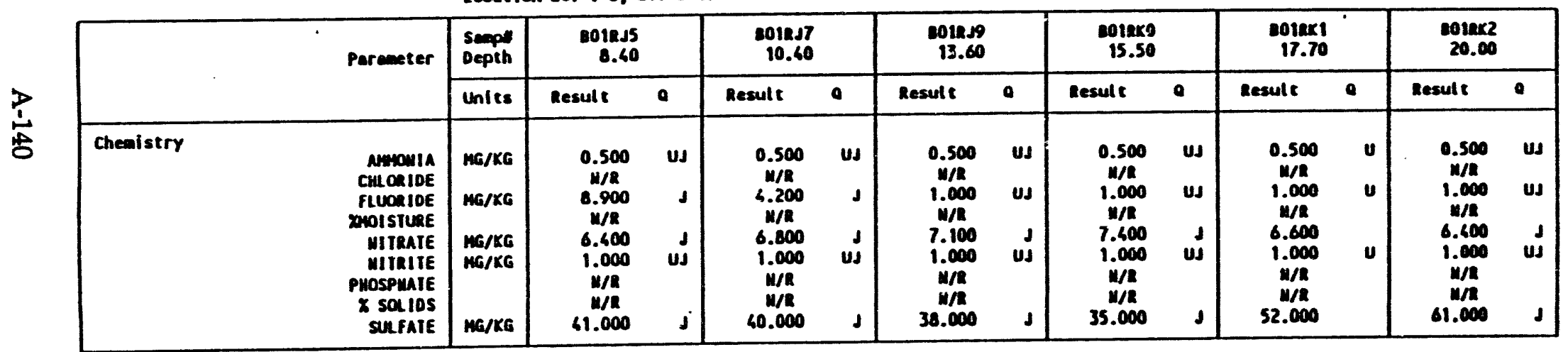




\begin{tabular}{|c|c|c|c|c|c|c|c|c|c|c|c|c|c|c|}
\hline & \multirow[t]{2}{*}{ Parameter } & \multirow{2}{*}{\begin{tabular}{l|} 
Sempl \\
Depth
\end{tabular}} & \multicolumn{2}{|l|}{$\begin{array}{r}0010 \times 3 \\
22.00 \\
\end{array}$} & \multicolumn{2}{|l|}{$\begin{array}{r}20101044 \\
24.00\end{array}$} & \multicolumn{2}{|l|}{$\begin{array}{r}2010 \times 5 \\
27.30\end{array}$} & \multicolumn{2}{|l|}{$\begin{array}{r}\text { conare } \\
33.00\end{array}$} & \multicolumn{2}{|l|}{$\begin{array}{r}0010 x 7 \\
30.00\end{array}$} & \multicolumn{2}{|c|}{$\begin{array}{l}0018 x 9 \\
52.00\end{array}$} \\
\hline & & & Result & a & Result & a & Result & a & Result & a & Result & - & result & - \\
\hline Chemistry & 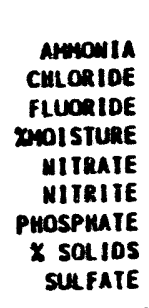 & $\begin{array}{l}\text { MG/KG } \\
\text { MG/KG } \\
\text { MG/KG } \\
\text { HG/KG } \\
\text { MG/KG }\end{array}$ & $\begin{array}{c}0.500 \\
W / R \\
1.000 \\
W / R \\
7.100 \\
1.000 \\
W / R \\
W / R \\
32.000\end{array}$ & us & $\begin{array}{c}0.500 \\
w / R \\
1.000 \\
w / R \\
5 / 8 \\
5.800 \\
1.000 \\
w / R \\
w / R \\
37.000\end{array}$ & US & $\begin{array}{c}0.500 \\
w / 2 \\
1.000 \\
w / 2 \\
5.300 \\
1.000 \\
w / 2 \\
w 1 / 8 \\
30.000\end{array}$ & $\begin{array}{l}\text { us } \\
\text { us } \\
\text { us }\end{array}$ & 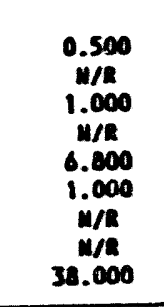 & us & $\begin{array}{c}0.500 \\
1 / 2 \\
1.000 \\
w / 2 \\
6 / 800 \\
1.000 \\
w / 2 \\
w / R \\
37.000 \\
\end{array}$ & $\begin{array}{l}\text { us } \\
\text { us } \\
\text { us }\end{array}$ & 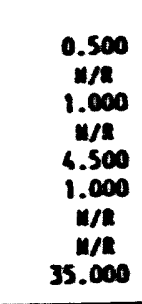 & $\begin{array}{l}\text { u } \\
\text { u }\end{array}$ \\
\hline
\end{tabular}


Lecation 307 T-3, 399-3-17

\begin{tabular}{|c|c|c|c|c|}
\hline & \multirow[t]{2}{*}{ Parameter } & $\begin{array}{l}\text { Semply } \\
\text { Depth }\end{array}$ & \multicolumn{2}{|c|}{$\begin{array}{r}00 \text { IRKs } \\
65.00\end{array}$} \\
\hline & & Units & Resule & 0 \\
\hline Chenistry & 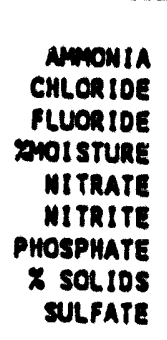 & $\begin{array}{l}\text { MG/Ko } \\
\text { MG/Ko } \\
\text { MG/Ko } \\
\text { Mo/Ko } \\
\text { MG/Ko }\end{array}$ & $\begin{array}{c}0.500 \\
N / R \\
1.000 \\
N / R \\
2.000 \\
1.000 \\
N / R \\
N / R \\
31.000\end{array}$ & $\begin{array}{l}u \\
u \\
u \\
u\end{array}$ \\
\hline
\end{tabular}



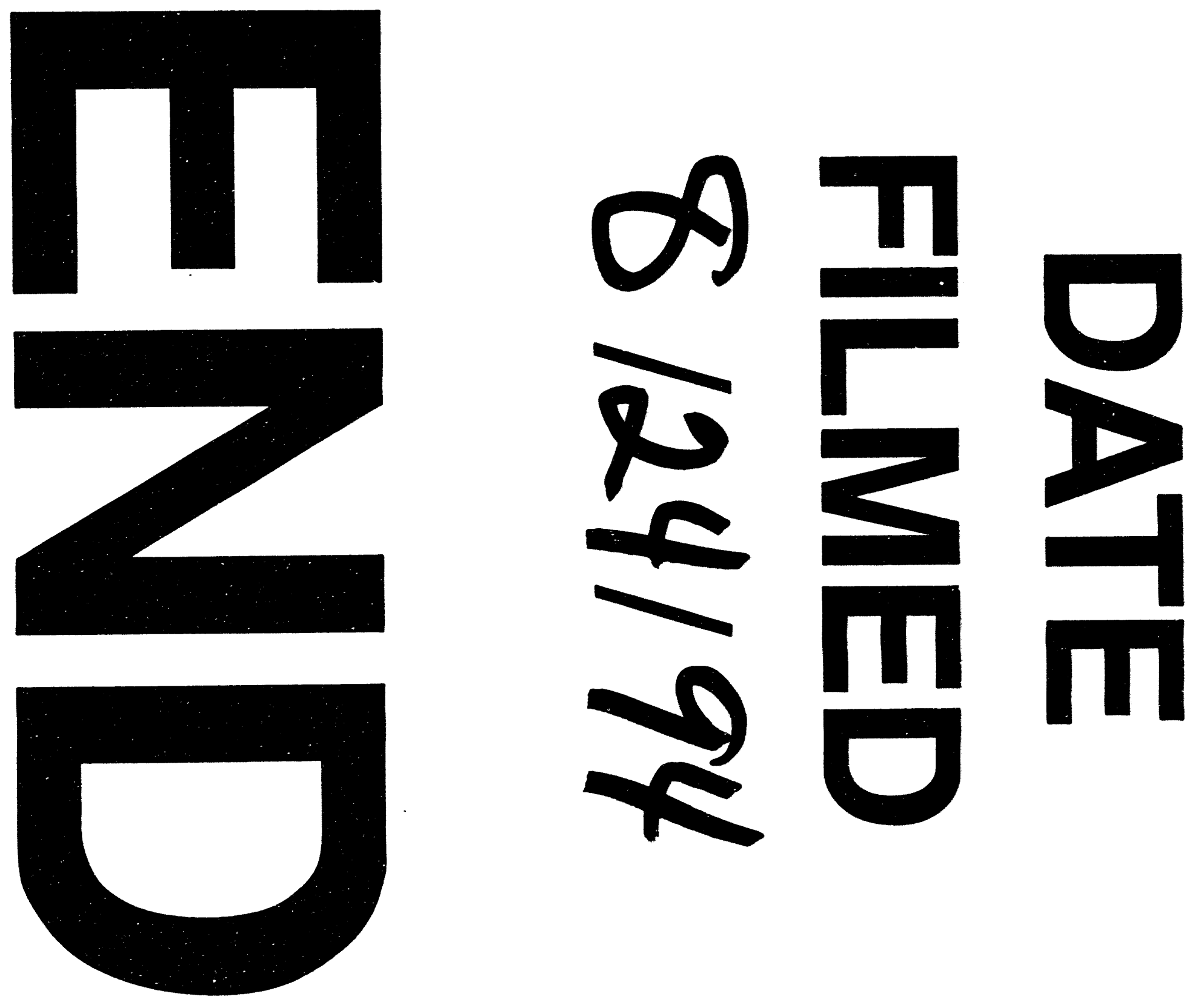


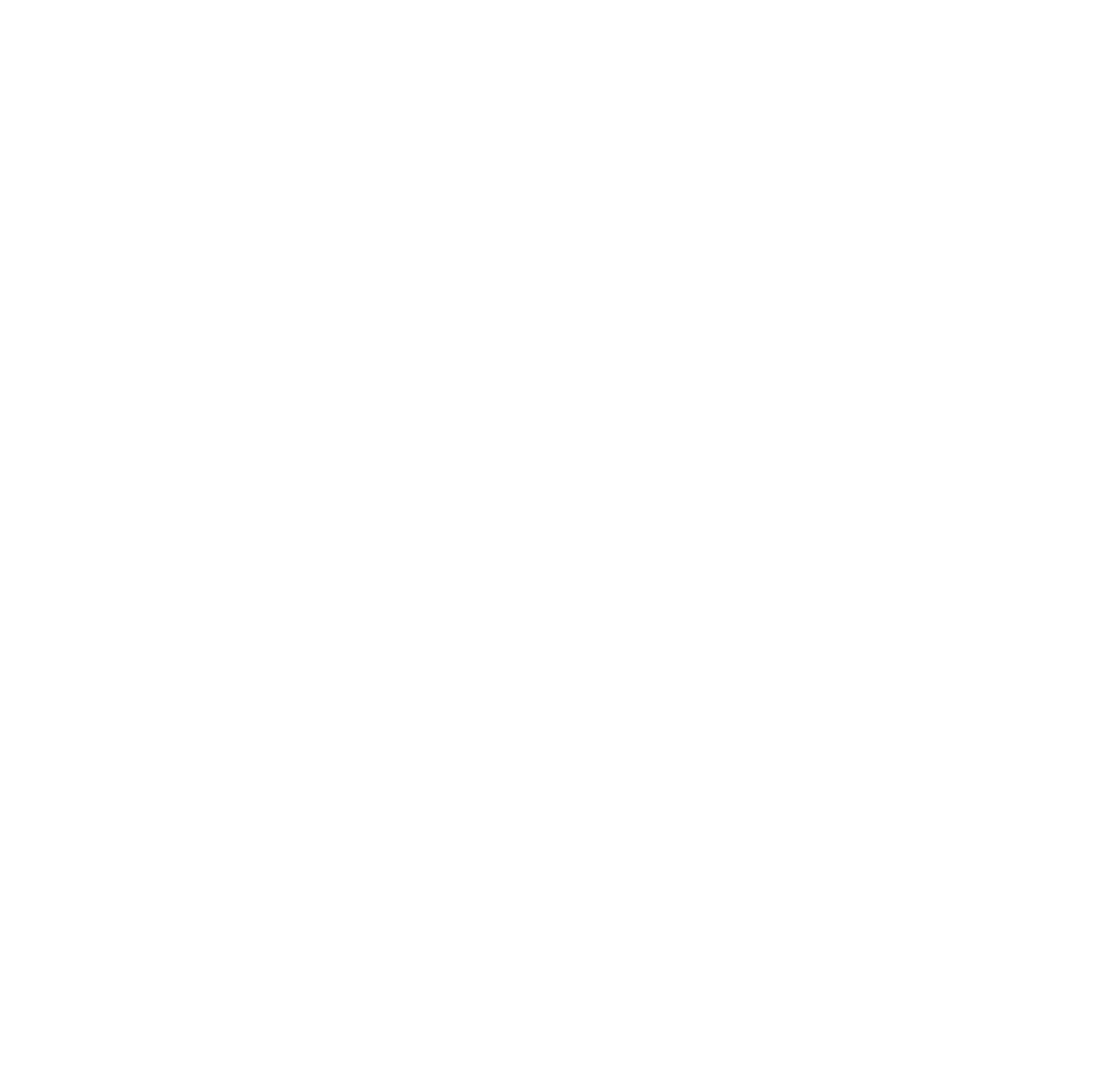

